US Army Corps

of Engineers ${ }_{\circledast}$

Engineer Research and

Development Center

\title{
Modernizing Environmental Signature Physics for Target Detection-Phase 3
}

Jay L. Clausen, C. Felt, M. Musty, V. Truong, S. Frankenstein,

March 2022

A. Wagner, R. Affleck, S. Peckham, and C. Williams

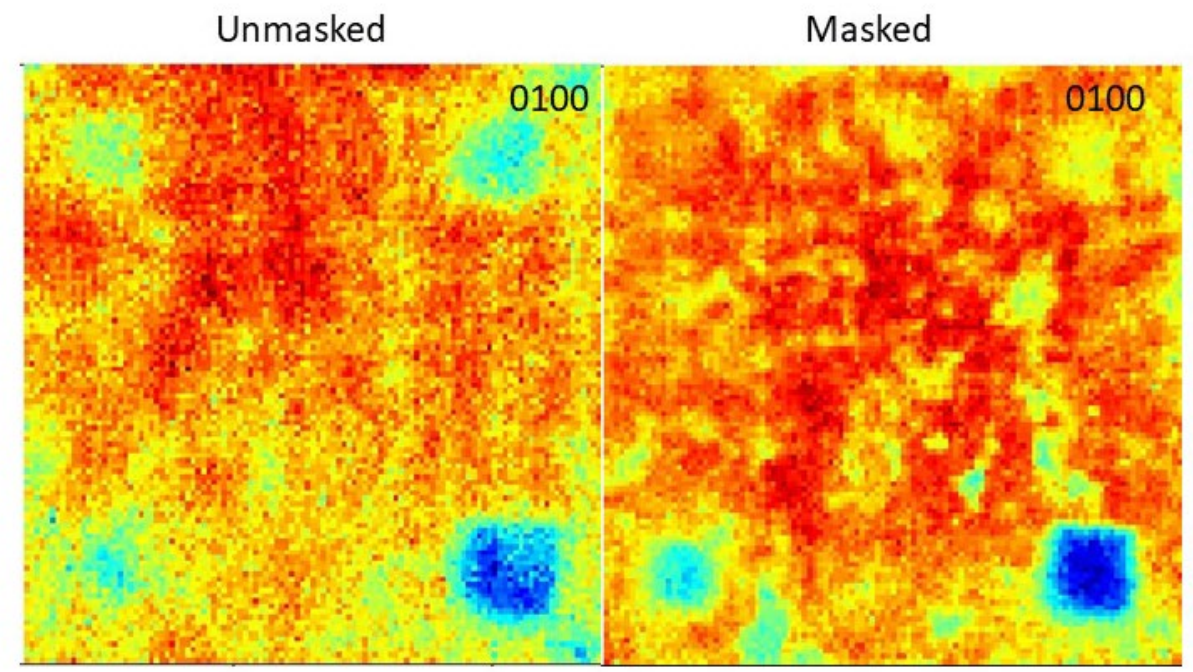

Buried Object

Detectability

By Time of Day

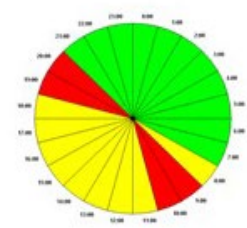

$\square$ Good

Acceptable Poor 
The U.S. Army Engineer Research and Development Center (ERDC) solves the nation's toughest engineering and environmental challenges. ERDC develops innovative solutions in civil and military engineering, geospatial sciences, water resources, and environmental sciences for the Army, the Department of Defense, civilian agencies, and our nation's public good. Find out more at www.erdc.usace.army.mil.

To search for other technical reports published by ERDC, visit the ERDC online library at https://erdclibrary.on.worldcat.org/discovery. 


\section{Modernizing Environmental Slgnature Physics for Target Detectlon-Phase 3}

Jay L. Clausen, C. Felt, M. Musty, V. Truong, S. Frankenstein, A. Wagner, R. Affleck, S. Peckham, and C. Williams.

U.S. Army Engineer Research and Development Center (ERDC)

Cold Regions Research and Engineering Laboratory (CRREL)

72 Lyme Road

Hanover, NH 03755-1290

Final Report

Approved for public release; distribution is unlimited.

Prepared for US Army Combat Capabilities Development Command Night Vision and Electronics Sensors Directorate (NVESD) AMSEL-RD-NV-RM-OPS 10221 Burbeck Road

Ft. Belvoir, VA 22060-5806 


\section{Abstract}

The present effort (Phase 3) builds on our previously published prior efforts (Phases 1 and 2), which examined methods of determining the probability of detection and false alarm rates using thermal infrared for buried object detection.

Environmental phenomenological effects are often represented in weather forecasts in a relatively coarse, hourly resolution, which introduces concerns such as exclusion or misrepresentation of ephemera or lags in timing when using this data as an input for the Army's Tactical Assault Kit software system. Additionally, the direct application of observed temperature data with weather model data may not be the best approach because metadata associated with the observations are not included. As a result, there is a need to explore mathematical methods such as Bayesian statistics to incorporate observations into models.

To better address this concern, the initial analysis in Phase 2 data is expanded in this report to include (1) multivariate analyses for detecting objects in soil, (2) a moving box analysis of object visibility with alternative methods for converting FLIR radiance values to thermal temperature values, (3) a calibrated thermal model of soil temperature using thermal IR imagery, and (4) a simple classifier method for automating buried object detection.

DISCLAIMER: The contents of this report are not to be used for advertising, publication, or promotional purposes. Citation of trade names does not constitute an official endorsement or approval of the use of such commercial products. All product names and trademarks cited are the property of their respective owners. The findings of this report are not to be construed as an official Department of the Army position unless so designated by other authorized documents.

DESTROY THIS REPORT WHEN NO LONGER NEEDED. DO NOT RETURN IT TO THE ORIGINATOR. 


\section{Contents}

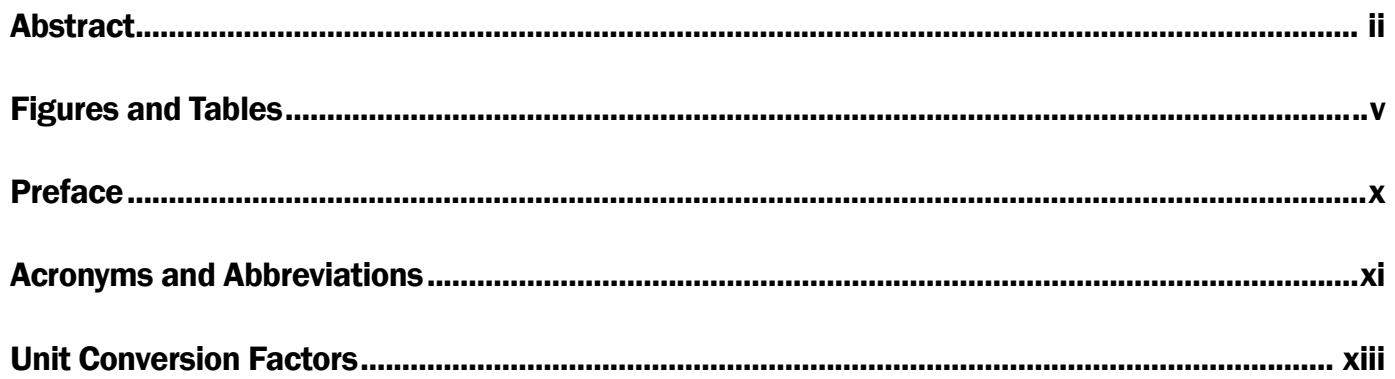

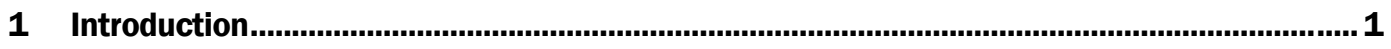



1.1.1 Environmental phenomenological impacts on thermal IR sensors................................ 2

1.1.2 Multi-sensor fusion including thermal IR .................................................................... 5

1.1.3 Yuma Proving Ground (YPG) Countermine Test Site comparison with Phase 2

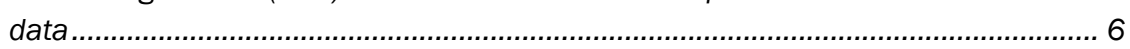

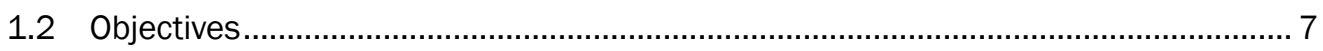

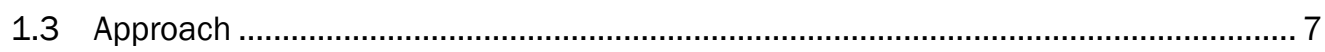

2 Display of Environmental Effects on Detecting Surface Targets............................................ 9

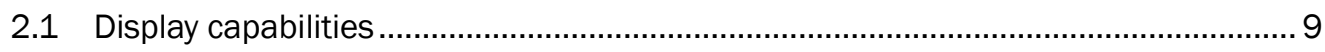

2.2 Case study......................................................................................................... 10

3 Moving Box Analysis of Buried Object Visibility ............................................................18

3.1 Convert FLIR camera radiances to thermal temperatures .......................................18

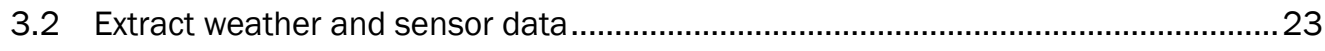

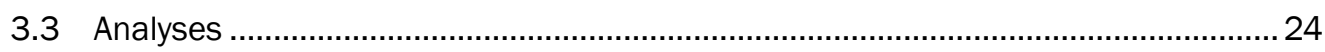

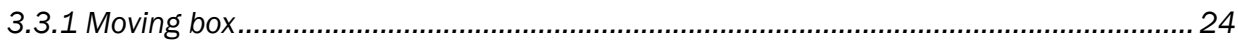

4 Assessment for Detecting Buried Objects in the Soil Using Multilevel Analyses ................27

4.1 Data for statistical analysis .......................................................................... 27

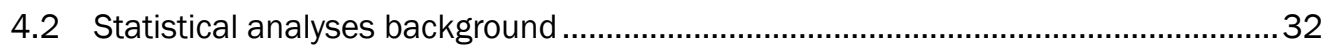

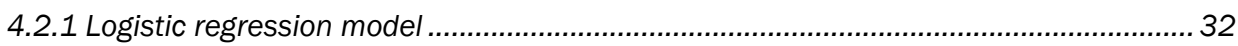

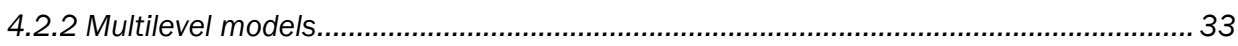

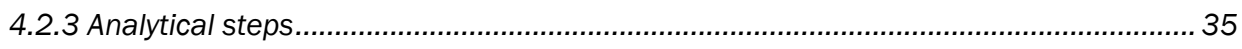

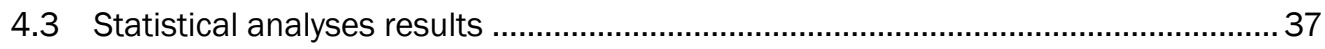

4.3.1 Model A: Object as a dependent variable ................................................................. 37

4.3.2 Model B: Soil temperature as outcome........................................................................ 42

4.3.3 Model C: Surface temperature with IR as outcome.................................................... 48

4.3.4 Model D: Soil moisture as outcome........................................................................ 51



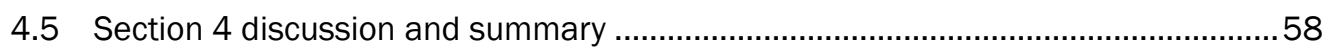

5 Thermal Model of Soil Temperature Using Thermal Imagery...........................................61

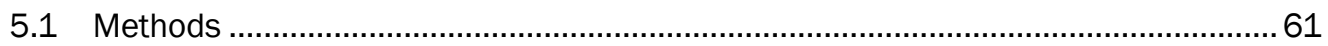






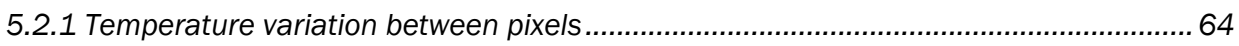

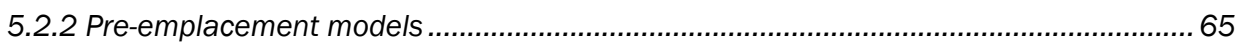



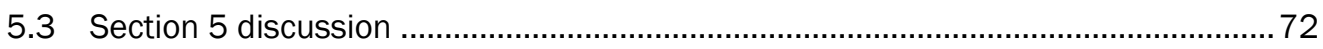

6 Automated Detection of Buried Objects: A Simple Classifier Method................................... 74

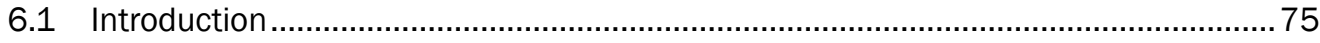

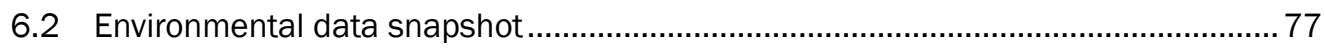

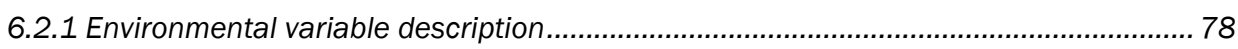

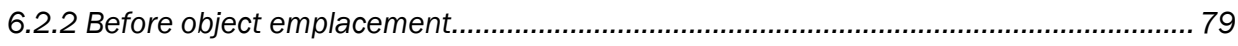

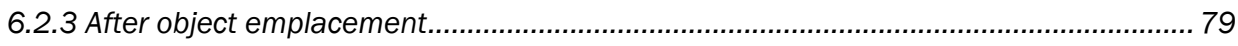

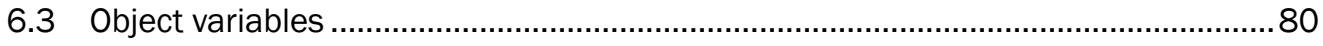

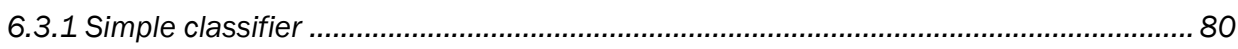

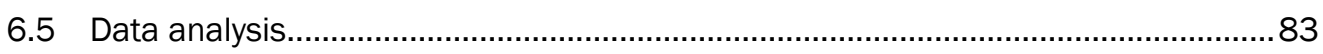

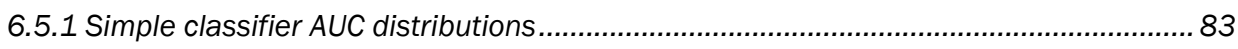

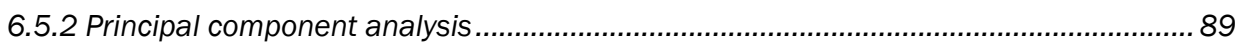

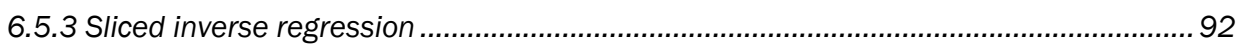



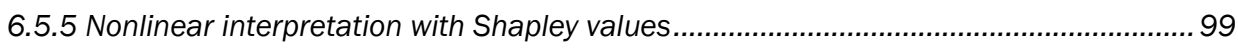

6.5.6 Nonlinear interpretation with global sensitivity analysis...........................................103

6.6 Future directions ..................................................................................... 105

6.6.1 Target variables .................................................................................................... 105

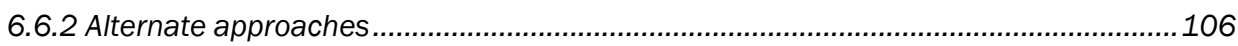

6.6.3 Time series analysis ............................................................................................. 106

6.6.4 Extrapolation and validation ............................................................................. 107

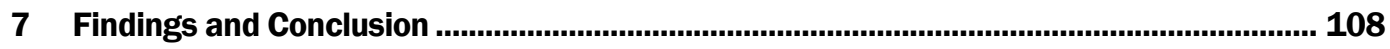

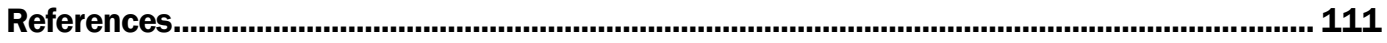

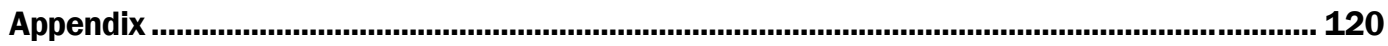



Appendix B. Classifiers and ROC analysis............................................................... 131

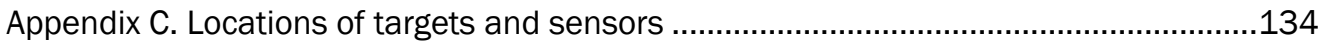

Temperature and moisture sensor locations.................................................................. 136 


\section{Figures and Tables}

\section{Figures}

Figure 1. The temperature over time produced by assuming the high and low temperatures follow a sinusoidal function (solid blue line). An observed temperature (blue dot on dashed line) is assumed and entered at a specified observation time. The observation has a corresponding time in the forecast model (green dot on solid line) and a time in which the forecasted temperature value would match that of the observation (red dot on solid line). The difference in time between the red and green dot represents the time phase shift of the model and the observation.

Figure 2. (a) The temperature over time produced by assuming the high and low temperatures follow a sinusoidal function. (b) The corresponding magnitude of temperature change $\left({ }^{\circ} \mathrm{C} / \mathrm{hr}\right)$ revealing a repetitive peak occurring approximately $6 \mathrm{hr}$ out of phase with the occurrence of the temperature extremes.

Figure 3. Computed stoplight color bar and magnitude of temperature change $\left({ }^{\circ} \mathrm{C}\right.$ per hour) revealing time periods of greatest temperature change which could permit easier detection of subsurface objects.

Figure 4. Image (a) minimum, (b) mean, and (c) maximum using the simple method with FLIR camera defaults (smpl1), measured weather (smpl2) or the complex method with defaults (cmpx1), weather ( $\mathrm{cmpx} 2$ ), weather plus actual camera height (cmpx3), and FLIR's proprietary software (FLIR).

Figure 5. Location of area of interest and buried objects with respect to the full image. $\mathrm{DP}=$ deep plastic object, $\mathrm{DM}=$ deep metal object, $\mathrm{SM}=$ shallow metal object, and $\mathrm{SP}=$ shallow metal object.

Figure 6. Probability of detection and probability of false alarms for the shallow plastic box using a difference in standard deviation cutoff of 1.5. Moving box overlap values are 0.0, 0.4, 0.6, and 1.0. Precipitation and air temperature are provided as points of reference. The dashed line in the upper left figure is at $0^{\circ} \mathrm{C}$.

Figure 7. Contrast Area plots (not drawn to scale) show the locations of undisturbed areas (small blue markers) and buried objects (solid and dashed blue and orange boxes with blue small markers in the middle). The four separate datasets for each object include the shaded zones for the (a) SM, (b) DM, (c) SP, and (d) DP of the CRREL test plot.

Figure 8. General plot layout (not drawn to scale) for the locations of IR imagery used in the analysis. The boxes denote 100 cells of $2 \mathrm{~cm} \times 2 \mathrm{~cm}$ cells of data above or on SM, SP, DP, and DM and undisturbed locations (blue).

Figure 9. Probability estimates of intercept and slope of the hourly IR surface and soil temperatures at $5 \mathrm{~cm}$ for the four contrasts (a) SM, (b) DM, (c) SP, and (d) DP.

Figure 10. Estimates of intercept and slope comparison for the hourly IR surface and soil temperature for the four contrasts (a) SM, (b) SP, (c) DM, and (d) SP.

Figure 11. ROC curves for the four contrasts (a) SM, (b) DM, (c) SP, and (d) DP at 1300 to 1345 (left) and at 1200 to1245 (right) for the surface temperature (IR_tir labeled as IR temp), soil temperature (SoilTemp5 labeled in the plot as Soil temp), and soil moisture (Soil15 renamed in the 
plot as Soil MC)

Figure 12. Hourly ROC curves comparisons for the (a) SM, (b) DM, (c) SP, and (d) DP contrasts between 15 July and 31 August 2018.

Figure 13. Model layout.

Figure 14. Overview of object (shallow plastic $=$ SP; deep plastic $=$ DP; shallow metal $=\mathrm{SM}$; deep metal $=\mathrm{DM}$ ) and thermistor placement at a depth of 5, 25, 45 , and $65 \mathrm{~cm}$.

Figure 15. Standard deviation of thermal imagery temperature of nine pixels at the control (C1, C2, C3, C4, and C5) and above each object (shallow plastic, deep plastic, shallow metal, and deep metal) and average for (a) preplacement of objects (1 June-12 July 2018) and (b) post-placement of objects (14 July-15 October 2018).

Figure 16. Simulated (BC: thermal imagery temperature and $0.05 \mathrm{~m}$ soil temperature) and measured temperatures at $0.05 \mathrm{~m}$ and $0.25 \mathrm{~m}$ for the (a) control (C3) and four object locations (b) SP, (c) DP, (d) SM, and (e) DM during the object pre-emplacement period (1 June-14 July 2018).

Figure 17. Pre-emplacement regression graphs for soil temperature (at 5 and $25 \mathrm{~cm}$ bgs) and simulated for $(a-c)$ Control 3, $(d-f)$ shallow plastic, $(g-i)$ deep plastic, $(j-l)$ shallow metal, and $(m-0)$ deep metal objects $(y=a+b x)$.

Figure 18. Simulated (boundary condition (BC) thermal imagery and soil temperature) and measured soil temperatures at $\mathrm{C} 3$ at soil depths (a) 5 $\mathrm{cm}$, (b) $15 \mathrm{~cm},(c) 25 \mathrm{~cm},(d) 35 \mathrm{~cm}$, (e) $45 \mathrm{~cm}$, and (f) $55 \mathrm{~cm}$ for the postplacement period (14 July-15 October 2018).

Figure 19. Regression plots of sensor temperature and simulated soil temperature for different soil depths at C3 (top panels) $(y=a+b x)$. Temperature difference between the measured temperature (sensor) and model (bottom panel) at C3.

Figure 20. Simulated ( $\mathrm{BC}$ thermal imagery and soil temperature) and measured soil temperatures at $5 \mathrm{~cm}$ and $25 \mathrm{~cm}$ bgs at the (a) SP, (b) DP, (c) SM, and (d) DM for the post-placement time period (14 July-15 October 2018). 72

Figure 21. A schematic of our main objectives.

Figure 22. The AOI with buried object locations with respect to the frame of reference defined by the IR image pixels.

Figure 23. AUC distributions (a) before target emplacement and (b) after target emplacement.

Figure 24. AUC distributions before and after target emplacement with AUC values computed using all targets. The Wasserstein distance between the distributions is 0.2191 .

Figure 25. AUC distributions before and after object emplacement with AUC values computed using (a) metal objects with Wasserstein distance 0.1667 and $(b)$ plastic objects with Wasserstein distance 0.2758 .

Figure 26. AUC distributions before and after target emplacement with AUC values computed using (a) deep objects with Wasserstein distance 0.2226 and $(b)$ shallow objects with Wasserstein distance 0.2123.

Figure 27. AUC distributions before and after object emplacement with AUC values computed using individual objects. The comparisons are for the (a) SP object with Wasserstein distance 0.4088 , (b) DP object with Wasserstein 
distance 0.1704, (c) SM object with Wasserstein distance 0.1221, and ()

$d$ DM object with Wasserstein distance 0.2879 .

Figure 28. The explained variance as the number of PCA components increases

when the PCA is computed using all environmental variables.

Figure 29. Scatter plot of the first PCA component against (a) AUC and (b) scatter plotusing three PCAcomponents with both plots colored by AUC usingall objects.

Figure 30. AUC distribution comparisons (using observations whose first PCA component is negative) for (a) an SM object with Wasserstein distance of 0.1198 and $(b)$ all objects with Wasserstein distance of 0.0395

Figure 31. Environmental data $X$ projected to the SIR latent space $W_{3}$ from Equation (16) (a) colored by AUC and (b) via $k$-means with $k=7$.

Figure 32. Environmental data $X$ projected to the SIR latent space $W_{2}$ from Equation (16) (a) colored by AUC and (b) via $k$-means with $k=7$.

Figure 33. Residual plots for XGBoost model using all environmental input variables. .98

Figure 34. Residual plots for an XGBoost model using the subset of applicable environmental input variables defined in Section 6.2.1.

Figure 35. Decomposition of an observed AUC based on (a) Shapley effects and (b) environmental variables ordered by feature importance measured by aggregating Shapley effects in relation to the baseline effect.

Figure 36. Shapley scatter plots for $(a)$ heat flux $\left(\mathrm{W} / \mathrm{m}^{2}\right)$ and $(b)$ the temperature $\left({ }^{\circ} \mathrm{C}\right)$ measured at a depth of $15 \mathrm{~cm}$ in location 20 within the plot.

Figure 37. Sobol (a) total sensitivity and (b) first order sensitivity sorted by variance ratio.

Figure 38. Highest second order Sobol indices associated with the average incoming solar radiation.

Figure B-1. A visualization of the dichotomy between $\alpha$ (type I error) and $\beta$ (type II error).

Figure C-1. Locations of temperature sensors at $5 \mathrm{~cm}$ with target locations in the sensor grid.

Figure C-2. Locations of temperature and moisture sensors at $15 \mathrm{~cm} \ldots \ldots \ldots \ldots \ldots \ldots \ldots \ldots \ldots \ldots \ldots \ldots \ldots . .138$

Figure C-3. Locations of temperature sensors at $25 \mathrm{~cm}$.............................................................139



Figure C-5. Locations of temperature sensors at $45 \mathrm{~cm}$............................................................ 141

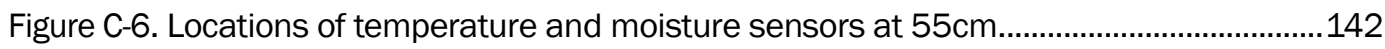

Figure C-7. Locations of temperature sensors at $65 \mathrm{~cm}$.............................................................143

\section{Tables}

Table 1. Subset of FLIR image metadata.

Table 2. Pixel location of area of interest and buried objects with respect to the full image. 23

Table 3. Four Square Method..

Table 4. Summary statistical models used in the analyses. 36

Table 5. Model A1 output of probability estimates and standard errors (values in parenthesis) from logistic regression model with intercept of Object as a 
dependent variable using the point layout datasets of the hourly average soil temperature and environmental variables.

Table 6. Model A2 results of probability estimates and standard errors (values in parenthesis) from logistic regression modeling with intercept of Object as a dependent variable using the $20 \mathrm{~cm} \times 20 \mathrm{~cm}$ area with the hourly average IR temperature, soil temperature, and soil moisture.

Table 7. Model A3 output of probability estimates and standard errors (values in parenthesis) from multilevel model procedures with intercept of Object as a dependent variable using GLIMMIX procedure and LAPLACE function. These estimates used the point layout datasets of the hourly average soil temperature and environmental variables.

Table 8. Model B1 results of probability estimates and standard errors (values in parenthesis) from multilevel model applying the PROC MIXED procedures for all contrasts with intercept of SoilTemp5 as a dependent variable. These estimates used the point layout datasets of the hourly average soil temperature and environmental variables.

Table 9. Model B2 output of probability estimates and standard errors (values in parenthesis) from 96 multilevel models of applying the PROC MIXED procedures aggregated based on time of day contrasts with intercept of soil temperature at $5 \mathrm{~cm}$ (SoilTemp) as a dependent variable

Table 10. Model $\mathrm{C}$ results of probability estimates and standard errors (values in parenthesis) from multilevel model of applying the PROC MIXED procedures aggregated based on time of the day contrasts with intercept of soil temperature at $25 \mathrm{~cm}$ (SoilTemp) as a dependent variable.

Table 11. Model D results of probability estimates and standard errors (values in parenthesis) from multilevel model of applying the PROC MIXED procedures aggregated based on time of the day contrasts with intercept of soil temperature radiance (IR_tir) as a dependent variable.

Table 12. Soil and object properties.

Table 13. AUC distribution table with baseline before target emplacement and test after object emplacement.

Table 14. AUC distribution for the baseline of all observations made after object emplacement against observations where the first PCA component is negative.

Table 15. AUC distribution table comparing the baseline of all observations made after object emplacement against observations bounded by the blue cutoff values in Figure 6.32a.

Table 16. AUC distribution for the baseline of all observations made after object emplacement against observations where heat flux variable FLX1 $\leq$ $-9.67$.

Table 17. AUC distribution for the baseline of all observations made after object emplacement against observations where heat flux variable FLX1 $\geq$ $-9.67$.

Table A-1. Descriptive statistics for dependent and independent variables of point layout datasets for shallow and deep metal contrasts for timeline 15 July31 August 2018.

Table A-2. Descriptive statistics for dependent and independent variables of point layout datasets for shallow and deep plastic contrasts for timeline 15 July-31 August 2018. 
Table A-3. Descriptive statistics of soil temperature observations at $25 \mathrm{~cm}$ for timeline 15 July-31 August 2018. ..............................................................................123

Table A-4. Descriptive statistics of soil temperature observations at $25 \mathrm{~cm}$ for timeline 15 July15-31 August 2018.

Table A-5. Descriptive statistics of IR temperatures above the shallow and deep metal contrasts for timeline 15 July-31 August 2018.

Table A-6. Descriptive statistics of IR temperatures above the shallow and deep plastic contrasts for timeline 15 July-31 August 2018.

Table A-7. Descriptive statistics of soil moisture observations at $15 \mathrm{~cm}$ for timeline 15 July-31 August 2018.

Table A-8. Descriptive statistics of soil moisture observations at $15 \mathrm{~cm}$ for timeline 15 July-31 August 2018.

Table B-1. Confusion matrix for a binary classifier. 


\section{Preface}

This study was conducted for the U.S. Army Combat Capabilities

Development Command (CCDC) C5ISR (Command, Control, Communications, Computers, Cyber, Intelligence, Surveillance, and Reconnaissance) Center, formerly known as the US Army Communications-Electronics Research, Development and Engineering Center (CERDEC) Night Vision and Electronic Sensors Directorate (NVESD) under Project 2040-20/21-8140B, "Modernizing Environmental Signature Physics for Target Detection." The program monitor was Dr. Robert Davis.

The work was performed by the Biogeochemical Sciences Branch (David Ringelberg, Acting Chief), the Terrestrial and Cryospheric Sciences Branch (Dr. John W. Weatherly, Chief), the Engineering Resources Branch (Ms. Caitlin Callahan, Chief), and the Force Projection and Sustainment Branch (Dr. Dorothea Lundberg, Chief), U.S. Army Engineer Research and Development Center, Cold Regions Research and Engineering Laboratory (ERDC-CRREL). At the time of publication, Dr. Steven Peckham was Acting Technical Director), Dr. George Calfas was Chief of the Research and Engineering Division, and Dr. Robert Davis was the Technical Director for Geospatial Research and Engineering/Military Engineering. The Deputy Director of ERDC-CRREL was Mr. David B. Ringelberg, and the Director was Dr. Joseph L. Corriveau.

COL Teresa A. Schlosser was the Commander of ERDC, and Dr. David Pittman was the Director. 


\section{Acronyms and Abbreviations}

\begin{tabular}{|c|c|}
\hline AOI & area of interest \\
\hline ATR & automatic target recognition \\
\hline AUC & area under curve \\
\hline bgs & below ground surface \\
\hline $\mathrm{CCDC}$ & Combat Capabilities Development Command \\
\hline CRREL & Cold Regions Research and Engineering Laboratory \\
\hline $\mathrm{C}_{5} \mathrm{ISR}$ & $\begin{array}{l}\text { Command, Control, Communications, Computers, Cyber, } \\
\text { Intelligence, Surveillance, and Reconnaissance }\end{array}$ \\
\hline $\mathrm{DM}$ & deep metal \\
\hline DoD & U.S. Department of Defense \\
\hline $\mathrm{DP}$ & deep plastic \\
\hline ERDC & Engineer Research and Development Center \\
\hline FAR & false alarm rate \\
\hline GPR & ground penetrating radar \\
\hline IR & infrared \\
\hline IVAS & integrated visual augmentation system \\
\hline LWIR & long-wave infrared \\
\hline LSM & land surface model \\
\hline PCA & principal component analysis \\
\hline $\mathrm{PD}$ & probability of detection \\
\hline
\end{tabular}




$\begin{array}{ll}\text { PFA } & \text { probability of false alarm } \\ \text { RGB } & \text { red, green, blue } \\ \text { SIR } & \text { sliced inverse regression } \\ \text { SM } & \text { shallow metal } \\ \text { SP } & \text { shallow plastic } \\ \text { TAK } & \text { tactical assault kit } \\ \text { YPG } & \text { Yuma Proving Ground }\end{array}$




\section{Unit Conversion Factors}

\begin{tabular}{|l|l|l|}
\hline Multiply & By & To Obtain \\
\hline degrees (angle) & 0.01745329 & radians \\
\hline degrees Fahrenheit & $(\mathrm{F}-32) / 1.8$ & degrees Celsius \\
\hline microns & $1.0 \mathrm{E}-06$ & meters \\
\hline square feet & 0.09290304 & square meters \\
\hline square inches & $6.4516 \mathrm{E}-04$ & square meters \\
\hline square miles & $2.589998 \mathrm{E}+06$ & square meters \\
\hline square yards & 0.8361274 & square meters \\
\hline yards & 0.9144 & meters \\
\hline
\end{tabular}




\section{Introduction}

For this and subsequent studies, the Engineer Research and Development Center (ERDC) / Combat Capabilities Development Command (CCDC) Command, Control, Communications, Computers, Cyber, Intelligence, Surveillance, and Reconnaissance ( $\mathrm{C}_{5}$ ISR) Test Bed located at Cold Regions Research and Engineering Laboratory (CRREL) in Hanover, NH, was utilized. The Phase 1 study (2016) involved development of a test plot with buried objects and sensors emplaced with as minimal soil disturbance as possible (Clausen et al. 2020). The findings showed that significant spatial and thermal temporal variability is caused by incoming solar radiation, meteorologically driven surface heat exchange, subsurface soil temperatures, density, soil moisture content, and surface roughness. Observations indicate surface soil temperature is extremely heterogenous and the amount of temperature variance changes throughout a 24-hour (hr) cycle. The spatial distribution of thermal anomalies also varies with no consistent pattern. Consequently, comparing two different days with identically environmental conditions for a given time within a $24 \mathrm{hr}$ cycle does not produce the same spatial background and target thermal response. We also found plastic objects heat and cool more rapidly than metal objects.

Phase 1 showed that time differencing of images could enhance detection of buried objects as compared to background noise, making it possible to find objects not visible in a single image frame (Clausen et al. 2020). The effect of time differencing on the visibility metrics was also investigated and indicated a normalizing contrast metric was most useful for object detection. Using the normalized contrast metric we found that the soil over the buried objects exhibited a much larger difference in derived IR temperature, as compared to background implying the buried objects would be discoverable during sunny and warmer days near solar noon. Deeply buried objects became easily visible when incoming solar radiation was $>900$ watts per square meter $\left(\mathrm{W} / \mathrm{m}^{2}\right)$.

A second study was conducted in 2018, referred to as Phase 2. Unlike Phase 1, Phase 2 involved removing the soil from the test plot area, homogenizing the material, then reapplying it along with buried sensors and objects representing targets of interest to the test plot into eight 
discrete layers (Clausen et al. 2021). Each layer was compacted to a uniform density consistent with the background undisturbed density. Homogenization of the test plot soil greatly reduced the micro-scale soil temperature variability simplifying the analysis of the data. The Phase 2 report focused on the design of the CRREL Test Bed along with data collection methodology and initial observations. Initial analyses were performed with a cross-correlation technique for determining the potential impact of meteorological factors and a simple contrast metric for detecting buried object visibility in thermal infrared (IR) imagery.

The research objective of Phase 3, this report, is to continue to improve our understanding of the environmental (soil and atmospheric) phenomenological properties affecting the temporal and spatial thermal variance of soils. The primary question being addressed, similar to the previous phases, is how to quantify the natural variance, or background noise, such that it can be subtracted from a disturbed area or object "signal" by taking into account the physical phenomenological material and atmospheric properties. The CRREL Test Plot configuration used to facilitate this effort was identical to the Phase 2 setup. As the data collection methods and Test Plot configuration were covered in detail in that report (Clausen et al. 2021), this investigation is primarily concerned with statistical analyses of the data gathered during Phase 2. Our hypothesis continues to focus on the interplay of variables and the thermal behavior of a homogeneous soil. Our results build on the Phase 2 study to provide an even deeper understanding of the physical variables influencing background noise and hindering thermal IR sensor performance.

\subsection{Background}

\subsubsection{Environmental phenomenological impacts on thermal IR sensors}

Thermal IR sensing does present some challenges, thus inspiring this work. Environmental factors that affect probability of detection (PD) and generate false alarm rate (FAR) are soil/material variability and porosity, soil disturbance, moisture migration/evaporation, and terrestrial radiant reflection and emittance. Diurnal thermal fluctuations due to changing solar input throughout the day give rise to thermal contrasts at the soil surface (Khanafer and Vafai 2002; van De Griend et al. 1985). Adding to this complexity is the additional influence of a buried object on both heat and moisture transfer (Pan and Mahrt 1987). Increases in soil moisture 
and density increase thermal conductivity, requiring specified calibration for the in situ soil under investigation (Smits et al. 2009). Under certain grain sizes, the effects of soil moisture can also be overshadowed by the latter porosity's impact on thermal conductivity, leading to drastic electromagnetic attenuation (Koh and Wakeley 2009). Understanding the phenomenology along with the magnitude of the interference from environmental parameters helps in quantifying these affects, thus improving current detection algorithms through a process of strategic elimination (Howington et al. 2019).

The trend in using active systems in thermal imagery does present some drawbacks as well. Potential scattering mechanisms that exist in the environment as well as the potential for sensor detection by an adversary need to be considered. When airborne particles are within a similar size as the wavelength, scattering efficiency increases. The particles in fogs, mists, and clouds range from 2-20 microns, making them an effective long-wave IR (LWIR) scatterer (Hudson and Hudson 1975). Furthermore, rainfall affects the physical and spectral properties of soils by separating silt particles away from larger particles in sand/silt soil mixture, which changes the surface particle size distribution (Ballard et al. 2013). Ballard discovered that the reflectance was most profound in the 8.1-9.2 micron band (thermal band). Ewing et al. (2020) went further in exploring the increase in the reststrahlen band with increasing sand/silt ratio with the maximum occurring at $80 \%$ sand but also demonstrated that mixed soil gives a reproducible decrease in reflectivity. Changes in temperature further affect the thermal conductivity and diffusivity of sand. For the silica sand samples investigated, Smits et al. (2013) discovered that the thermal conductivity and diffusivity increased most noticeably for temperatures greater than $50^{\circ} \mathrm{C}$, in agreement with independent empirical models. But the accurate prediction of surface temperature is hindered by surface wetness and thermal inertia (Price 1985), leading to complex thermal variability. However, several properties of the soil can be rapidly analyzed with near-IR reflectance spectroscopy, which is the dominant analytical technique used for grain and forage quality assessments (Chang et al. 2001).

Additionally, water molecules are IR active, thus atmospheric transmissions in the thermal IR band are degraded by molecular rotational transitions from water vapor or broadening effects from hydrogen-bonding interactions in the cases of liquid water (Gorman 1957; 
Raqueno et al. 2008). Van Dam et al. (2003) concluded that water content does indeed affect thermal signatures, though the correlation didn't have a quantifiable linear relationship. Instead, accounting for the properties of disturbed and undisturbed soil can help better detect buried objects (Koenig et al. 2008). The presence of a buried object disturbs the heat and diffusion parameters of the soil, enabling anomaly detection (Frost 2006). However, it now is generally believed that it is the disturbance of the soil and alteration of the soil properties that give rise to the thermal signature. Thermal contrasts are manifested as a result of three effects: (1) the object itself likely has different thermal properties than the soil it has replaced, which may result in differential heat transport and a thermal expression at the surface; (2) disturbing a soil normally changes its density and thermal properties, also leading to a surface expression of physical temperature; and (3) disturbing a soil redistributes finer particles to the ground surface, which, for some soil compositions, changes their spectral reflectance (Howington 2020). Interestingly, the surface effect remains for weeks after the disturbance and enhances the contrast induced by the buried object, a phenomenon also observed in our earlier work (Clausen et al. 2021 and 2020).

Waldemar et al. (2012) extended the work of Hong et al. (2002) and Van Dam et al. (2003). They used physical measurements and numerical modeling techniques to observe the effects that varying moisture levels and the density of soil have on buried-object detection. Howington et al. (2012) believe that hydrogeological variability must be considered on a similar scale of the sensor's image pixels (a few centimeters) to isolate and quantify the near-surface process interactions to produce an accurate simulation. High resolution synthetic thermal image production has helped in optimizing explosive detection (Peters et al. 2007), along with using mathematical statistical modeling on the heat and water flow in the soil (Lamorski et al. 2002), but accuracy is highly dependent on knowledge of the external conditions in order to adjust the criteria used. Predicting expected surface temperatures is complicated by the nonlinear movement of water within soil and changes in surface temperature during the drying process (Frost 2006). Thành et al. (2011) introduced inverse problem setting and used a least-squares minimization approach for buried object detection. Their group confirmed that the deeper the object is buried, the less accurate will be the reconstruction, while larger objects produced greater accuracy upon reconstruction. However, their model didn't tackle inhomogeneous soil and complex shapes. 
Understanding the relationship between meteorological conditions and soil properties is paramount to detect objects buried in soil and predict sensor performance. An analytical solution requires extensive knowledge of the buried material, surface composition, water content, and humidity of the environment. This multivariate and multilayered problem is thus limited without quantifying the environment absolutely. Without knowing all the parameters involved, van Dam (2003) has stated that it's impossible to predict the thermal signatures accurately.

\subsubsection{Multi-sensor fusion including thermal IR}

However, at the opposite end of the spectrum, the sensor application community has been exploring "active" ways to increase PD of buried objects by utilizing a combination of various sensing technologies or a multi-sensor approach, sometimes referred to as sensor fusion. Recently, the resolution and contrast of thermal imaging has been shown to be significantly improved by utilizing polarized thermal sensors, which has the potential to simultaneously decrease background clutter while increasing contrast and producing highly resolved fused images (Baur et al. 2018; Jarrod et al. 2019). Disturbed regions in comparison to the surrounding exhibit higher degrees of linear polarimetric contrast (Gurton and Felton 2012). Gurton's team utilized a spinning-achromatic-retarder platform for LWIR polarimetric imaging, resulting in improved detectability, regardless of the geographic location or soil type. Similarly, Abbott et al. (2020) created an effective detection algorithm by utilizing red, green, blue (RGB) imagery for translating non-corresponding LWIR/RGB datasets due to the lack of standalone IR annotated data. Pinar et al. (2017) added in a forward-looking ground penetrating radar (GPR) and light detection and ranging (LIDAR) in unison with their visible and thermal camera, improving the signal-to-noise-ratio and thus increasing the buried target classification accuracy.

Active systems such as GPR have been coupled to a thermal camera's superior standoff capabilities to complement GPR's higher detection performance (Malof and Collins 2016; Hendrickx et al. 2006). As a standalone sensor modality, GPR has been gaining popularity in landmine detection (Shaw et al. 2016; Kelly et al. 2019). The polarization effects on GPR have been studied extensively (Dogaru and Le 2016, Sagnard 2017), as have the effects from soil moisture (Miller et al. 2004; Miller et al. 2002). Miller's (2004) field measurements showed that increasing moisture around a nonmetallic buried object can improve detection in 
sand and silt soils due to the increasing contrast in the soil's bulk dielectric constant as the water content increases. Clay soils, on the other hand, showed the opposite effect, with greater attenuation with increasing water content, limiting GPR's potential as a standalone sensor modality.

Other techniques in buried object detection include the use of microwave generators (Deans 2001) and IR heaters (Yao et al. 2019), which are utilized simultaneously with a passive thermal sensor to compliment and stimulate thermal variability. Yao's team used an IR lamp adjusted to 800 $\mathrm{W} / \mathrm{m}^{2}$ to mimic the natural diurnal cycle of Earth in a controlled indoor environment. Li et al. (2020) took a different approach by observing how buried objects impacted the acoustic-to-seismic coupling efficiency by tracking the changes in acoustic impedance. Similarly, von Deimling et al. (2015) utilized a parametric echosounder system to investigate the angular response of the backscatter on underwater targets. Finally, Kabessa et al. (2016) introduced a standoff biosensor capable of buried object detection. Their detection scheme consists of genetically engineered live bacterial sensor strains that produce a dose-dependent amount of green fluorescent protein in the presence of explosives' vapors, which are then remotely detected by a phase-locked optoelectronic sampling system.

\subsubsection{Yuma Proving Ground (YPG) Countermine Test Site comparison with Phase 2 data}

The EDRC-CRREL team, along with personnel from ERDC-GSL and the Desert Research Institute, performed a field test over an 11-day period at the YPG test site during February 2020 to expand on CRREL's Phase 2 findings by gathering data on buried object visibility from a secondary location besides the CRREL test plot. The findings from the test effort are reported in Musty et al. (2021). In the test, four kinds of buried objects were used-shallow metal and plastic objects and deep metal and plastic objects - with size, composition, and depths identical to the CRREL Phase 2 test plot configuration (Clausen et al. 2021). In addition, the visibility of surface objects was tested using a total of six hockey puckshaped objects, with three composed of plastic and three of aluminum, all painted a uniform desert brown color to visually blend in with the surface soil.

The same FLIR camera was used for both the CRREL test plot in Phase 2 and the YPG test site. The thermal imagery collected for the buried objects exhibited a similar behavior for both data sets. In general, object visibility 
predictably followed solar loading, with times coinciding with crossover yielding the least visibility. Soil moisture tended to confound this effect, with increased soil moisture typically resulting in reduced visibility. In the YPG dataset, solar loading, soil moisture, air temperature, and soil conductivity were the most impactful environmental variables on object visibility. This finding differs slightly from the CRREL test plot findings in that air temperature appeared to contribute more impact to visibility at CRREL than at YPG. Variables with little effect on visibility across both datasets include wind speed, humidity, and pressure.

\subsection{Objectives}

Our earlier work explored the soil and atmospheric phenomenological properties affecting temporal and spatial thermal variance of soils and targets. The goal of the present study is to improve subsurface object detection through an increase of PD and reduction in FAR for thermal IR sensors. To accomplish this goal our efforts have been focused on identifying, for a given soil and meteorological state, the physical variables that significantly contribute to soil temperature variations between disturbed and undisturbed soil. The question being addressed is how to quantify the natural variance "noise" such that it can be subtracted from a disturbed area "signal" by considering the physical phenomenological material and atmospheric properties. Our hypothesis is the thermal behavior of a soil is a function of several inter-dependent variables such as material density, moisture content, reflectance, surface texture, and atmospheric thermal loading. The information on environmental phenomenology will be incorporated into a pre-processor for improving automatic targeting recognition (ATR) software performance used by the Army.

\subsection{Approach}

Building on our prior research and data collected in Phase 2, we continued to investigate the phenomenological properties controlling sensor performance for detecting buried objects. Our approach toward meeting the research objective involved the characterization of soil along with changing atmospheric conditions to assess the impact of the spatial and temporal variance on IR buried object detection. To identify the environmental variables impacting ATR performance utilizing thermal IR data, we took a multi-pronged approach. We investigated if the buried object thermal anomalies from an IR sensor can be discerned within an 
environment of spatial soil property variance and changing weather conditions utilizing continuous data collection from the CRREL Test Plot through (1) multivariate analyses for detecting objects in soil, (2) a moving box analysis of object visibility with alternative methods for converting thermal IR radiance values to thermal temperature values, (3) a calibrated thermal model of soil temperature using thermal IR imagery, and (4) a simple classifier method for automating buried object detection. Further, we expanded our dataset beyond the CRREL Test Plot through a 2-week field test at YPG, the full findings for which will be presented in a subsequent report. 


\section{Display of Environmental Effects on Detecting Surface Targets}

The displaying of the location, spatial relationships, and information related to surface-based targets is an essential element of battlespace success. The proper acquisition and geo-located display of pertinent targets and associated battlefield information for an area of operation dramatically enhances a commander's situational awareness and reduces time for decision-making. In this section, the research program examines methods by which assessment of surface characteristics associated with targets can be geolocated and rapidly displayed to enhance understanding of the battlefield environment.

\subsection{Display capabilities}

The National Defense Strategy 2018 (McInnis 2018) emphasized that new technology will change the character of war. While many technological developments occur in the commercial sector, laboratories will also have to access and utilize these emerging capabilities.

One such emerging technology, the Integrated Visual Augmentation System (IVAS), is being designed to incorporate head, body, and weapon technologies on individual soldiers. The IVAS system features advanced eyewear connected to a small computer that places simulated images in a soldier's view of real-world environments. It is a single platform that the warfighter can use to fight, rehearse, and train that provides increased lethality, mobility, and situational awareness necessary to overmatch current and future adversaries. The system includes squad-level combat training capability for repeated iterations of training and rehearsals.

Part of the early artificial intelligence/machine learning capability within the IVAS system are planning and operational tools utilizing the Tactical Assault Kit (TAK) software. The TAK software is a federally and U.S. Department of Defense (DoD)-adopted geospatial infrastructure and military situational awareness application with a plugin architecture allowing developers to add functionality. Specifically, TAK is an opensource, map-based situational awareness software with applications that function across multiple computational platforms-including Android, Linux, and Windows-providing tactical capabilities for military operations. The TAK core is a software development foundation with an 
extensible framework through which plugin architectures can provide enhanced capabilities for specific mission sets (direct action, combat decision-making, law enforcement, disaster response, etc.). Further, this software development framework addresses the nearly identical Standard and Shareable Geospatial Foundation requirements regarding content, formats, and services such as georeferenced imagery and geographic feature data, supporting the Geopackage data file profile, and supporting web map services.

Our research effort utilized the Windows-based TAK software (DoD's WinTAK), a version of the TAK geospatial development software that functions on a personal computer operating system installed on a laptop or tablet. WinTAK provides ground users and unmanned aircraft systems (UAS) pilots a meaningful, geospatial site picture and inter-operates with other DoD situational awareness tools including SpyGlass, RaptorX, FalconView, and other legacy systems. WinTAK's standalone capabilities include moving map functions independent of the network and allowing maps to be loaded during mission pre-planning or execution phase. The WinTAK software is extensible, allowing users to add their own custom functionality and employ multiple types of analysis software systems simultaneously.

\subsection{Case study}

Fundamental to thermal target detection is the three-dimensional heat equation. This equation can be solved to demonstrate how heat diffuses through a medium of differing thermal conductivity and density. This calculation demonstrates that if an object is inserted into a medium of contrasting material (i.e., buried in soil) and a uniform thermal load is applied to the soil surface, the thermal response of the buried object initially lags that of the surrounding soil. However, after several hours the object will start to heat up faster than the surrounding soil, presumably due to differences in heat capacity. That is, the ground near the object will not warm as quickly as the surrounding terrain, providing a thermal difference that can be sufficient to identify buried targets. The periods in which target detection most easily occurs are when air and soil surface temperatures are rapidly increasing or decreasing. This knowledge then permits users of target detection to exploit the changing environment to find buried objects along a designated route. 
Changes to surface temperature are well understood and associated with energy propagation to and from the land surface. The ways in which energy can propagate is described by the surface energy balance equation, which is defined as:

$$
R_{n}=H+\lambda E+G
$$

where

$\mathrm{R}_{\mathrm{n}}=$ the net radiation which is the summation of downward shortwave and long-wave radiation,

$\mathrm{H}=$ sensible heat flux emitted from the surface $\left(\mathrm{W} / \mathrm{m}^{2}\right)$,

$\lambda \mathrm{E}=$ latent heat flux from the surface $\left(\mathrm{W} / \mathrm{m}^{2}\right)$, and

$\mathrm{G}=$ heat conducted away from the surface $\left(\mathrm{W} / \mathrm{m}^{2}\right)$.

The left-hand side of the surface energy balance equation denotes the energy inputs to the surface, which is also called the radiative forcing term or gain. The right-hand side of the equation denotes energy outputs from the surface, or loss terms.

Typically, these surface fluxes require multiple sets of observation equipment installed at a particular site, making precise observations across an area of interest nearly impossible. However, a mean area solution to this equation can be obtained through utilization of a numerical weather prediction model. These models compute each component of the energy balance equation in a Land Surface Model (LSM) to describe the physical boundary between the surface and the atmosphere. The LSM provides estimates of the surface sensible and latent heat fluxes, the reflected short-wave radiation, and the upward long-wave radiation, upward long-wave radiation being interchangeable with the model estimate of local soil surface temperature and surface emissivity. Hence, the variations in model-predicted air surface temperature should be adequate to estimate periods of rapid ground temperature changes and by extension when surface and subsurface targets are more easily identifiable.

A model of soil surface temperature change needs to be generated to identify periods of rapid temperature change. Here a simple model will be utilized-rather than forecast products from a high-resolution numerical weather prediction system-to validate the concept. This simple 
temperature model will exploit findings of previous research which recognized that observed surface temperature tends to follow a sinusoid pattern over a 24-hour period (de Wit 1978; Baker et al. 1989). Supporting this concept, the sinusoidal model of de Wit (1978) provided a reasonable representation of the observed diurnal surface temperature variation (Baker et al. 1989). The formula describing the temporal temperature evolution divides the day into two periods, one from the time of morning minimum temperature to the time of afternoon maximum, the other covering the remainder of the $24 \mathrm{hr}$ day. Each portion approximates the temperature as a cosine curve, scaling the respective period to a range between $o$ to $\pi$ radians, resulting in consecutive half waves with amplitude equal to the total temperature difference for the select day with the whole function fluctuating about a center line connecting consecutive extrema:

$$
T(t)=\frac{T_{h}+T_{i}}{2} \pm \frac{T_{h}+T_{i}}{2} \cos \left[\pi \frac{\left(t-t_{1}\right)}{t_{h}-t_{i}}\right]
$$

where warming periods utilize summation and cooling periods subtraction and

$$
\begin{array}{ll}
T(t) & =\text { The surface air temperature at time t } \\
T_{h} & =\text { The warmest temperature for the day } \\
T_{i} & =\text { The coolest temperature of the day } \\
\pi & =3.14159 \\
t & =\text { time [units=hr in model] } \\
t_{1} & =\text { start time } \\
t_{h} & =\text { time of warmest temperature } \\
t_{i} & =\text { time of the coolest temperature. }
\end{array}
$$

Since this formulation is based upon local time instead of Coordinated Universal Time (UTC), the temperature is phase shifted by roughly 90 degrees. That is, the maximum temperature occurs near 1800 and the low around 060o. Given that $\cos (x+\pi / 2)=-\sin (x)$, the surface temperature can be represented by a sine function. Further, it is recognized the temperature change is typically not as smooth as a single sine function and higher order modes might exist due to weather fronts, associated clouds, and precipitation. However, for this exercise we choose to represent the variations of surface temperature, $T$, with the function 


$$
T=T_{m}+T_{a} \sin (\omega t+\phi)
$$

where

$$
\begin{aligned}
T_{m} & =\text { mean temperature }\left(\sim 12^{\circ} \mathrm{C}\right) \\
T_{a} & =\text { daily temperature change amplitude }\left(10^{\circ} \mathrm{C}\right) \\
\omega & =\text { frequency of temperature oscillation (one day period); } \\
\phi & =\text { time phase shift for temperature to represent the local high } \\
& \text { and low readings of the day for a location in the Eastern } \\
& \text { Time Zone in UTC. }
\end{aligned}
$$

The temperature equation produces an oscillating sine wave pattern plotted as the blue line in Figure 1. The temperature falls during the morning hours until shortly after sunrise when there is a leveling before a period of warming until close to the highest temperature of the day in the afternoon, where the temperature remains nearly constant before cooling for the remainder of the day.

Figure 1. The temperature over time produced by assuming the high and low temperatures follow a sinusoidal function (solid blue line). An observed temperature (blue dot on dashed line) is assumed and entered at a specified observation time. The observation has a corresponding time in the forecast model (green dot on solid line) and a time in which the forecasted temperature value would match that of the observation (red dot on solid line). The difference in time between the red and green dot represents the time phase shift of the model and the observation.

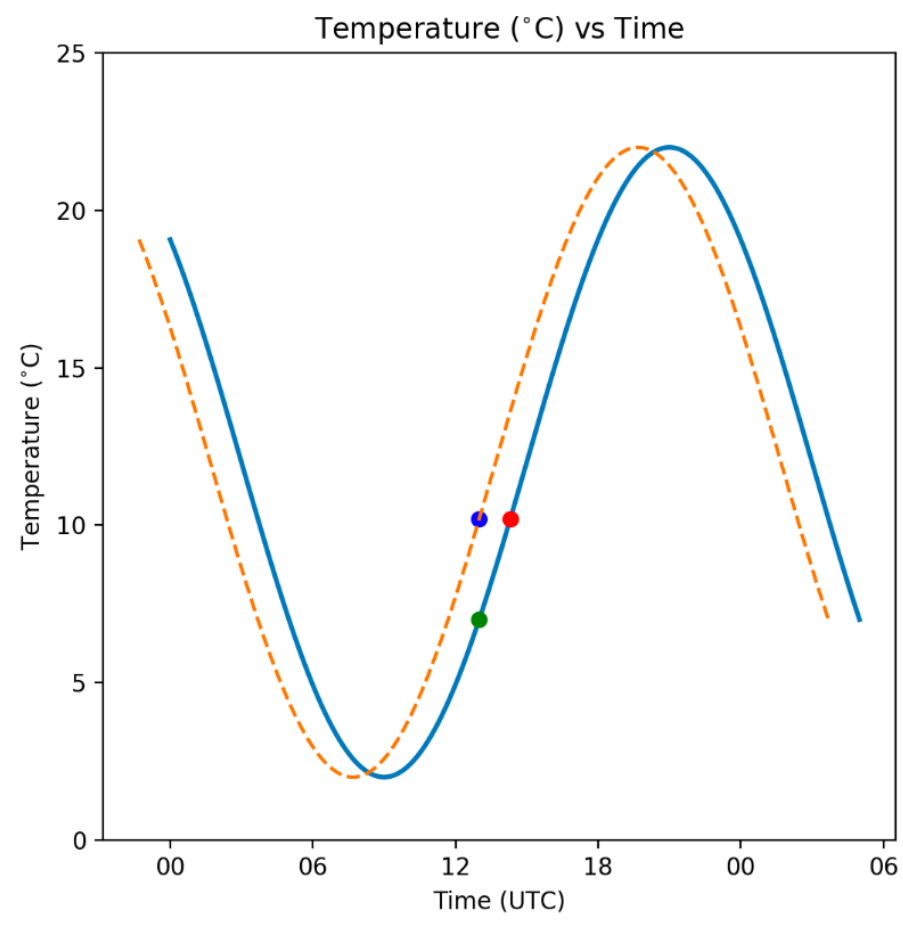


Next, we examine temperature data to determine the periods of greatest temperature change. Given the rate of temperature change is described by the slope of the curve, one can compute the first derivative of $T$ with respect to time, which is the equation that describes the temperature change over time.

$$
\frac{d T}{d t}=T_{a} \omega \cos (\omega t+\phi)
$$

where

$$
\begin{aligned}
d T & =\text { change in temperature, } \\
d t & =\text { change in time, } \\
T_{a} & =\text { amplitude of the diurnal temperature change }\left(\mathrm{T}_{\mathrm{h}}-\mathrm{T}_{\mathrm{i}}\right), \\
\omega & =\text { frequency of temperature oscillation (one day period) } \\
T & =\text { time [same units as frequency], and } \\
\phi & =\text { time phase shift for temperature to represent the local high } \\
& \text { and low readings of the day for a location in the Eastern } \\
& \text { Time Zone in UTC. }
\end{aligned}
$$

Recognizing it is the rate of temperature change that enhances the detection of buried targets and not the magnitude of temperature increase or decrease, the magnitude of the first derivative is obtained (Figure 2). 
Figure 2. (a) The temperature over time produced by assuming the high and low temperatures follow a sinusoidal function. (b) The corresponding magnitude of temperature change $\left({ }^{\circ} \mathrm{C} / \mathrm{hr}\right.$ ) revealing a repetitive peak occurring approximately $6 \mathrm{hr}$ out of phase with the occurrence of the temperature extremes.
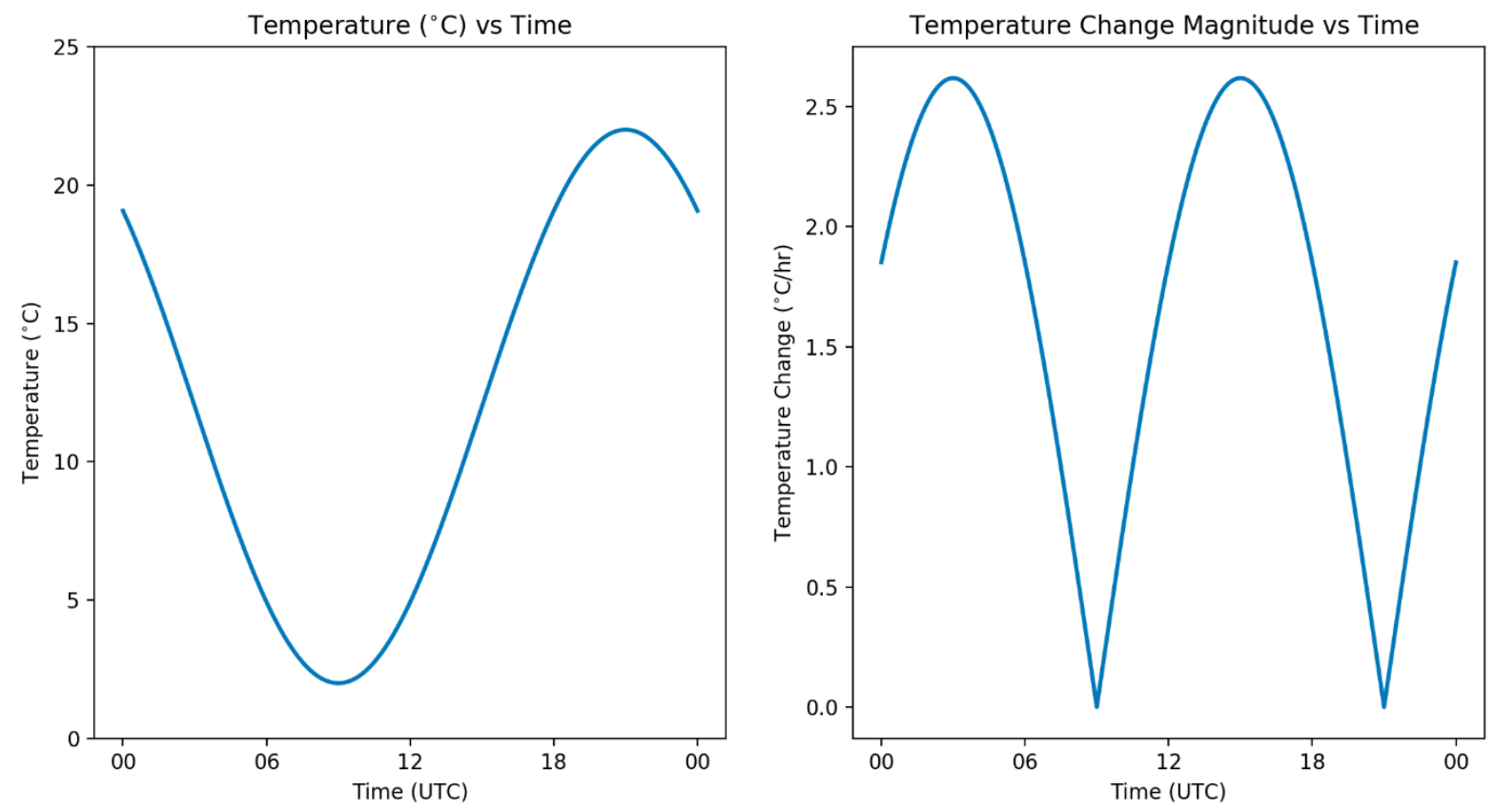

The temperature change magnitude equation reveals that cyclic changes take place each day over several hours. Further, the magnitude of temperature change is almost never zero but instead rapidly increases and decreases during a $7 \mathrm{hr}$ time interval in which the temperature change is greater than $1.2^{\circ} \mathrm{C} / \mathrm{hr}$, symmetric around a $2 \mathrm{hr}$ block of time during which the temperature change is greater than $2.2^{\circ} \mathrm{C} / \mathrm{hr}$. Given these events occur twice daily and at regular intervals, they can be tested against observational data.

The rate of temperature change can be separated into three periods and depicted with a stoplight color scheme for ease of comprehension. The stoplight colors highlight when air temperature is rapidly changing, which should lead to better conditions for surface target detection. In this study, we define temperature change magnitudes $<1.2^{\circ} \mathrm{C} / \mathrm{hr}$ as low probability (red) and change magnitudes $>2.2^{\circ} \mathrm{C} / \mathrm{hr}$ as high probability (green). Between the two is a region of medium probability of detection (yellow) (Figure 3). With these definitions there are clear demarcations between the detection probability time periods, with the emphasis falling in a time interval of approximately $4 \mathrm{hr}$ centered at 0300 UTC (associated with evening cooling) and 1500 UTC (morning warming). 
Figure 3. Computed stoplight color bar and magnitude of temperature change $\left({ }^{\circ} \mathrm{C}\right.$ per hour) revealing time periods of greatest temperature change which could permit easier detection of subsurface objects.



An issue with implementing this temperature model is the smooth, cyclical nature of the solution compared to physical observations exhibiting higher order modes of a typical weather forecast model (Ek et al. 2003). The testing of the algorithm through utilization of numerical weather prediction forecast data would need to be conducted as these data are a forthcoming addition to the TAK software system (e.g., ATAK, WinTAK). A primary concern with forecast data inclusion is the coarse temporal resolution of the numerical products and these products' ability to resolve the microscale phenomena contributing to the changes in temperature. Weather forecast data are often provided in hourly intervals, which can cause the exclusion or misrepresentation of ephemera or introduce lags in timing.

Furthermore, the method of including temperature observations needs to be examined in greater detail. The direct application of observed temperature data with weather model data is not necessarily appropriate as metadata about the observation are not included. The local observation might be at a different altitude than the model and could be over a different land usage (vegetation type and coverage) or soil texture. This 
suggests a need for exploring mathematical methods of incorporating observations into models such as Bayesian statistics. Accordingly, Sections 3-6 comprise a multi-approach analysis of the CRREL Test Plot data. 


\section{Moving Box Analysis of Buried Object Visibility}

In this subtask, the buried object PD using a moving box and simple foursquare method of analysis was examined. Because the FLIR camera used in Phase 1 and Phase 2 utilizes a proprietary algorithm for producing thermal IR radiance values (FLIR Systems 2015), we first investigated two alternative methods of converting FLIR camera radiances to thermal temperatures.

\subsection{Convert FLIR camera radiances to thermal temperatures}

FLIR proprietary software and camera default values were used to convert the measured radiances to surface thermal temperatures for all of the Phase 1 and Phase 2 analyses. The camera measures the total radiation $\left(W_{\text {tot }}, \mathrm{W} / \mathrm{m}^{2}\right)$ given by (FLIR Systems 2015)

$$
W_{t o t}=\varepsilon \tau W_{o b j}+(1-\varepsilon) \tau W_{r e f l}+(1-\tau) W_{a t m}
$$

where

$$
\begin{aligned}
& \varepsilon=\text { surface emissivity, } \\
& \tau=\text { atmospheric transmission, } \\
& W_{\text {obj }}=\text { object radiation }\left(\mathrm{W} / \mathrm{m}^{2}\right), \\
& W_{\text {refl }}=\text { reflected radiation }\left(\mathrm{W} / \mathrm{m}^{2}\right), \text { and } \\
& W_{\text {atm }}=\text { atmospheric radiation }\left(\mathrm{W} / \mathrm{m}^{2)} .\right.
\end{aligned}
$$

The first term quantifies the radiation emitted from the object, the second represents the reflected surface emittance, and the third is the background radiation emitted from the surrounding atmosphere. The radiation follows Stefan-Boltzman's law such that

$$
W=\varepsilon \sigma T^{4}
$$

where:

$$
\begin{aligned}
\varepsilon= & \text { surface emissivity, } \\
\sigma= & 5.670374419 \times 10^{-8} \mathrm{~W} / \mathrm{m}^{2} \mathrm{k}^{4} \sigma=5.670374419 \times 10^{-8} \mathrm{~W} / \mathrm{m}^{2} \mathrm{~K} 4, \\
& \text { which is the Stefan-Boltzman constant, and } \\
T= & \text { temperature }(\mathrm{K}) .
\end{aligned}
$$


The image metadata and radiance values were extracted within MATLAB using ExifTool software. Since the algorithm that FLIR Systems uses is unpublished, two other methods were investigated. For clarity in the following discussion, these are called simple and complex.

The simple method uses the method presented by Harvey (2013). It assumes the object distance, $O D$, is o $\mathrm{m}$ and the external optics transmission, IRT, is 1.o. The resulting formulation is

$$
\begin{gathered}
T_{o b j}=\frac{R_{1}}{\ln \left(\frac{R_{1}}{R_{2}\left(W_{o b j}+O\right)}+F\right)} \\
W_{o b j}=\frac{s-(1-\varepsilon) W_{r e f l}}{\varepsilon} \\
W_{r e f l}=\frac{R_{1}}{R_{2}\left(\exp \left[\frac{B}{\left.T_{r e f l}\right)}\right]-F\right)}-O
\end{gathered}
$$

The variables $R_{1}, R_{2}, F$, and $O$ are various Planck constants and $S$ is the camera measured radiance. The values used are part of the metadata associated with the thermal images and are listed in Table 1. 
Table 1. Subset of FLIR image metadata.

\begin{tabular}{|l|l|}
\hline FLIR Parameter & default value \\
\hline File Modify Date & \\
\hline Emissivity, $\boldsymbol{\varepsilon}$ & 0.92 \\
\hline Object Distance, OD & $2.0 \mathrm{~m}$ \\
\hline Reflected Apparent Temperature, $T_{\text {refl }}$ & $20.0^{\circ} \mathrm{C}(293 \mathrm{~K})$ \\
\hline Atmospheric Temperature, $T_{\text {atm }}$ & $20.0^{\circ} \mathrm{C}(293 \mathrm{~K})$ \\
\hline IR Window Temperature, $T_{\text {IRwin }}$ & $20.0^{\circ} \mathrm{C}(293 \mathrm{~K})$ \\
\hline IR Window Transmission, IRT & 1.00 \\
\hline Relative Humidity, RH & $50.0 \%$ \\
\hline Planck R1, R1 & 18112.691 \\
\hline Planck B, B & 1455 \\
\hline Planck F, F & 1 \\
\hline Atmospheric Transmission Alpha 1, $A T_{A 1}$ & 0.006569 \\
\hline Atmospheric Transmission Alpha 2, $A T_{A 2}$ & 0.012620 \\
\hline Atmospheric Transmission Beta 1, $A T_{B 1}$ & -0.002276 \\
\hline Atmospheric Transmission Beta 2, $A T_{B 2}$ & -0.006670 \\
\hline Atmospheric Transmission X, $A T_{X}$ & 1.900000 \\
\hline Lens Model & FOL4 \\
\hline Planck 0, O & -7882 \\
\hline Planck R2, R2 & 0.011804782 \\
\hline Raw Thermal Image Width & 320 \\
\hline Raw Thermal Image Height & 240 \\
\hline & \\
\hline
\end{tabular}

The complex method uses the algorithm presented by Tattersall (2019) in his R code "raw2temp." Specifically:

$$
\begin{gathered}
T_{o b j}=\frac{B}{\ln \left(\frac{R_{1}}{R_{2}\left(W_{o b j}+O\right)}+F\right)} \\
W_{o b j}=S / \tau_{1} / I R T / \tau_{2}-W_{a t m 1 *}-W_{a t m 2 *}-W_{\text {win* }}-W_{\text {refl } 1 *}-W_{\text {refl2* }} \\
W_{a t m 1}=W_{a t m 2}=\frac{R_{1}}{\left(R_{2} \exp \left[\frac{B}{T_{a i r}}\right]-F\right)}-O
\end{gathered}
$$




$$
\begin{aligned}
& W_{\text {atm } 1 *}=\left[\left(1-\tau_{1}\right) / \varepsilon / \tau_{1}\right] W_{a t m 1}, W_{a t m 2 *}=\left[\left(1-\tau_{2}\right) / \varepsilon / \tau_{1} / I R T / \tau_{2}\right] W_{a t m 2} \\
& W_{\text {refl1 }}=W_{\text {refl2 }}=\frac{R_{1}}{\left(R_{2} \exp \left[\frac{B}{T_{r e f l}}\right]-F\right)}-O \\
& W_{\text {refl1* }}=[(1-\varepsilon) / \varepsilon] W_{r e f l 1}, W_{r e f l 2 *}=\left[R_{w i n} / \varepsilon / \tau_{1} / I R T\right] W_{r e f l 2} \\
& W_{\text {win }}=\frac{R_{1}}{\left(R_{2} \exp \left[\frac{B}{T_{I R \text { win }}}\right]-F\right)}-O, W_{\text {win } *}=\left[\varepsilon_{\text {win }} / \varepsilon / T_{1}\right] W_{\text {win }} \\
& \tau_{1}=\tau_{2}=A T_{x} \exp \left[-\sqrt{0.5 O D}\left(A T_{A 1}+A T_{b 1} \sqrt{H_{2} O}\right)\right] \\
& +\left(1-A T_{x}\right) \exp \left[-\sqrt{0.50 D}\left(A T_{A 2}+A T_{B 2} \sqrt{H_{2} O}\right)\right] \\
& \mathrm{H}_{2} \mathrm{O}=0.01 R H \exp \left[1.5587+0.06939 T_{\text {air }}-0.00027816 T_{\text {air }}^{2}+6.8455\right. \\
& \left.\times 10^{-7} T_{\text {air }}^{3}\right] \\
& \varepsilon_{\text {win }}=1-I R T, R_{\text {win }}=0
\end{aligned}
$$

where

$R_{\text {win }}$ is the reflection of the camera lens,

$T_{\text {air }}$ is the air temperature $(\mathrm{K})$,

$\boldsymbol{\varepsilon}_{\boldsymbol{w i n}}$ is the lens emissivity, and

* subscripts indicate attenuated values.

All remaining parameters are defined in Table 1. All temperatures in the equation are in Kelvin.

The sensitivity of the calculated thermal temperatures resulting from using either the simple or complex methods with both the default temperatures, relative humidity, object distance, and object emissivity versus measured, or calculated values, was investigated. For the latter, it was assumed that $T_{\text {refl }}=T_{\text {air }}$. To account for the darkening of the soil when it is wet, the emissivity was changed from 0.92 to 0.98 based on the work of Chung and Horton (1987). The dry value of 0.92 is consistent for silty sand, Unified Soil Classification System (USCS) soil type SM (silty sand, sandy-silt; Frankenstein and Koenig, 2004). Since the surface soil wetness was not measured, the increased emissivity is applied during a precipitation event until half an hour after the event. 
The results as well as values calculated using FLIR's proprietary software are shown in Figure 4 for five images taken on the morning of 24 September 2018. It is evident from this figure that FLIR uses the simple method, Equation (7), with default weather values. If the FLIR default weather-related parameters are used, there is an approximately $2^{\circ} \mathrm{C}$ difference in thermal temperature between the two methods with the simple method being warmer than the complex one. If instead the measured weather data are used, there is almost no difference between the two methods. The highest temperatures are calculated if the actual distance between the camera and the soil surface, $4.27 \mathrm{~m}$, is used instead of the $2.0 \mathrm{~m}$ default value (green dash-dot line in Figure 4).

Figure 4. Image (a) minimum, $(b)$ mean, and (c) maximum using the simple method with FLIR camera defaults (smpl1), measured weather (smpl2) or the complex method with defaults (cmpx1), weather (cmpx2), weather plus actual camera height (cmpx3), and FLIR's proprietary software (FLIR).
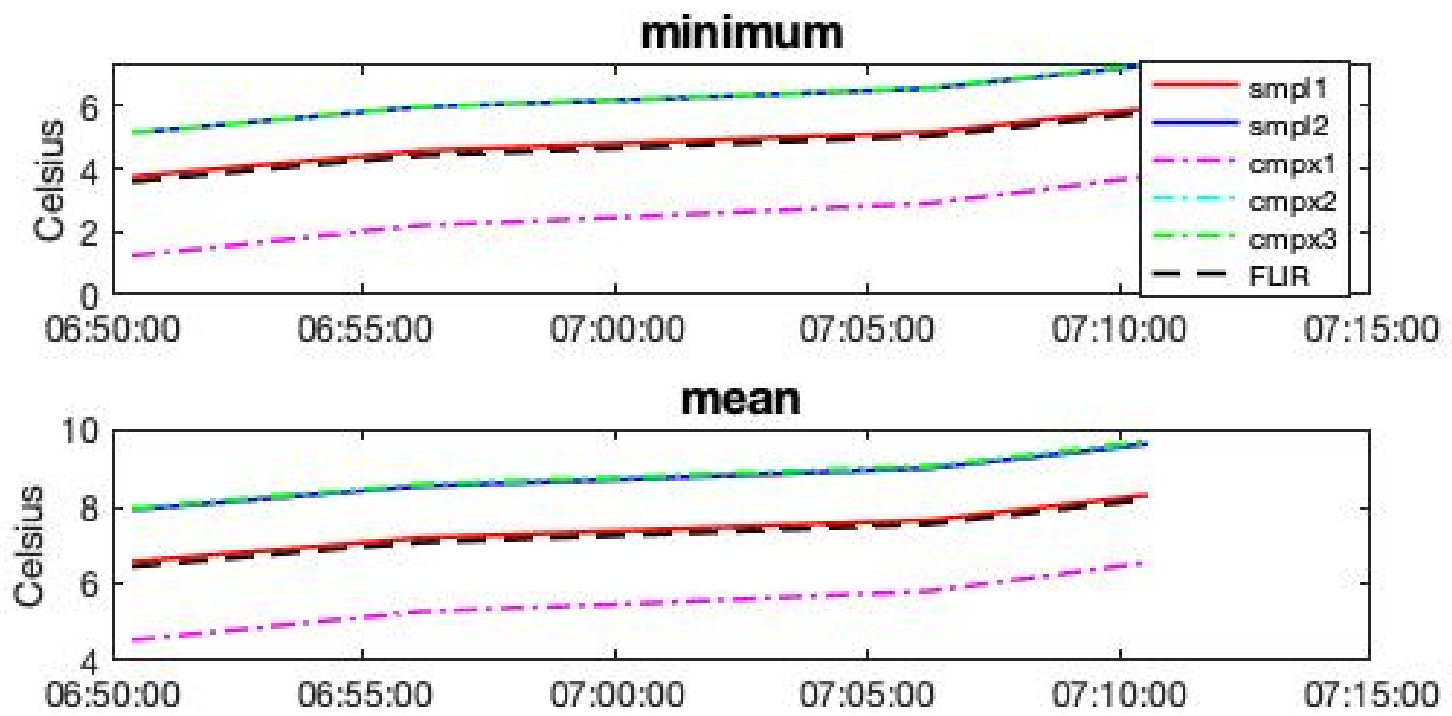

maximum

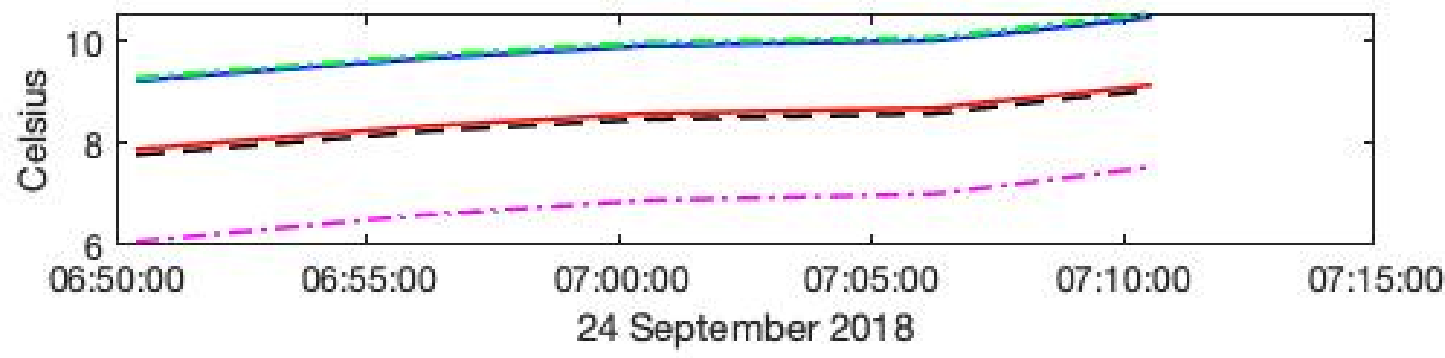




\subsection{Extract weather and sensor data}

After converting the radiances to thermal temperatures, the next step before analyses could begin is to extract the associated weather. The image data is collected at 5-minute intervals while the meteorological and sensor information is averaged and recorded every 15 minutes. Two different recording devices are used, resulting in an offset between the two data sets. The sensors of interest for this initial analysis are the thermistors installed at 5 and $15 \mathrm{~cm}$ below ground surface (bgs) and the Campbell Scientific CS655 probes at $15 \mathrm{~cm}$ bgs. The pixel locations of these sensors are listed in Table 2. In order to compare on a pixel-by-pixel basis, the sensor data were gridded to the plot using MATLAB's scattered interpolant function with the natural-neighbor method. Next, the sensor and meteorological data were extracted to the image times using linear interpolation. The image times used were the "File Modify Date" from the associated FLIR metadata.

Table 2. Pixel location of area of interest and buried objects with respect to the full image.

\begin{tabular}{|l|l|}
\hline Location & (column, row) \\
\hline upper left corner of image & $(0,0)$ \\
\hline lower right of image & $(320,240)$ \\
\hline upper left corner of plot & $(108,62)$ \\
\hline lower right corner of plot & $(222,174)$ \\
\hline upper left corner of DP & $(117,70)$ \\
\hline lower right corner of DP & $(135,87)$ \\
\hline upper left corner of DM & $(194,70)$ \\
\hline lower right corner of DM & $(212,87)$ \\
\hline upper left corner of SM & $(117,150)$ \\
\hline lower right corner of SM & $(135,167)$ \\
\hline upper left corner of SP & $(194,150)$ \\
\hline lower right corner of SP & $(212,167)$ \\
\hline object dimensions & $(18,17)$ \\
\hline thermal couples at $5 \mathrm{~cm}$ & $\begin{array}{l}(126,79),(164,79),(203,79),(126,118),(164,118), \\
(203,118),(126,158),(164,158),(203,158),(156,108), \\
(172,108),(160,114),(168,114),(160,122),(168,122), \\
(156,126),(108,118),(172,126),(108,158),(126,174), \\
(164,174)\end{array}$ \\
\hline thermal couples at 15 cm & $\begin{array}{l}(126,136),(126,140),(126,144),(126,148),(181,158), \\
(185,158),(189,158),(193,158),(156,108),(172,108),\end{array}$ \\
\hline
\end{tabular}




\begin{tabular}{|l|l|}
\hline Location & (column, row) \\
\hline & $\begin{array}{l}(160,114),(168,114),(160,122),(168,122),(156,126), \\
(172,126)\end{array}$ \\
\hline CS655 probes at $15 \mathrm{~cm}$ & $\begin{array}{l}(126,79),(164,79),(203,79),(126,118),(164,118), \\
(203,118),(203,148),(164,158),(126,158),(108,118), \\
(108,158),(126,174),(164,174)\end{array}$ \\
\hline
\end{tabular}

\subsection{Analyses}

\subsubsection{Moving box}

Since the exact location of a buried object is unknown a priori, analysis was performed for a moving box having dimensions $5 \times 5$ pixels, beginning in the upper left and ending in the lower right corner of the plot. The percent overlap of the box with each of the buried objects was recorded. To do this, the position of the boxes in the image needed to be determined. In doing this, it was found that a grayscale image was preferable to a falsecolored one. Figure 5 shows the location of the test plot within the full image as well as the four buried objects. The objects are equal sized, $40 \mathrm{~cm}$ $\times 40 \mathrm{~cm} \times 20 \mathrm{~cm}$. Two of the boxes are plastic $(\mathrm{P})$, two are metal $(\mathrm{M})$, and one of each material is buried such that the tops are either $5 \mathrm{~cm}(\mathrm{~S})$ or 25 $\mathrm{cm}$ (D) below the surface. The pixel locations of the objects are listed in Table 2.

The difference between the moving box and plot means, standard deviations, and a visibility index (VI) are calculated. The VI is defined as

$$
V I=\frac{s t d_{5 \times 5}-s t d_{\text {plot }}}{s t d_{5 \times 5}+s t d_{\text {plot }}}
$$

where

$s t d_{5 \times 5}$ is the standard deviation of the box and $s t d_{\text {plot }}$ is the standard deviation of the plot. 
Figure 5. Location of area of interest and buried objects with respect to the full image. DP = deep plastic object, $\mathrm{DM}=$ deep metal object, $\mathrm{SM}=$ shallow metal object, and SP = shallow metal object.

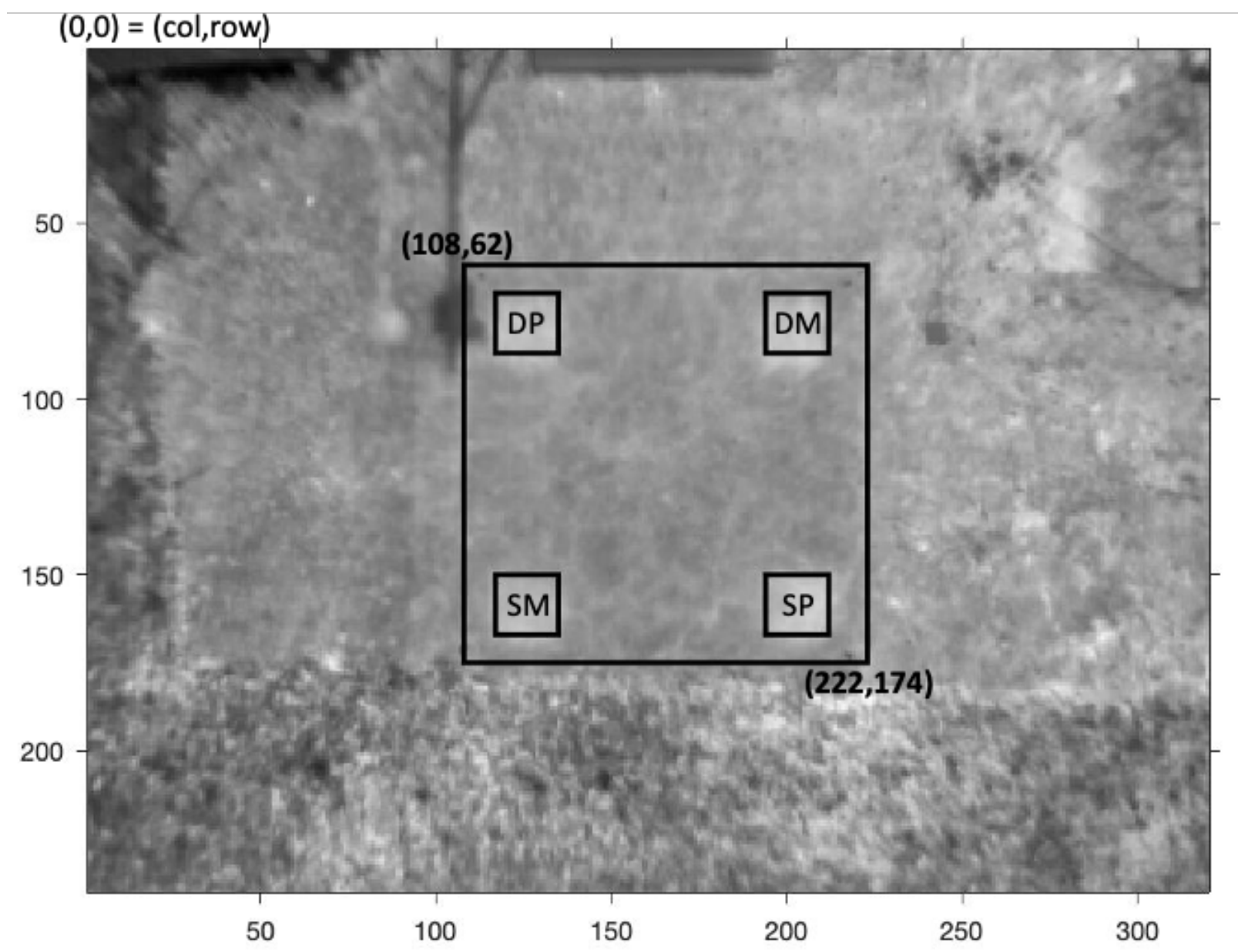

As a first attempt to establish the statistical significance of differences in the standard deviation, mean temperature difference, and VI, a simple four-square method is used. The criteria are defined in Table 3.

Table 3. Four Square Method

\begin{tabular}{|l|l|l|}
\hline Statistical Test & Over Box & Not Over Box \\
\hline larger or equal to criteria & $\begin{array}{l}\text { true positive }(\mathrm{YY}) \\
\text { no error }\end{array}$ & $\begin{array}{l}\text { false positive }(\mathrm{YN}) \\
\text { type II error }\end{array}$ \\
\hline less than criteria & $\begin{array}{l}\text { false negative (NY) } \\
\text { type I error }\end{array}$ & $\begin{array}{l}\text { true negative (NN) } \\
\text { no error }\end{array}$ \\
\hline
\end{tabular}

$\mathrm{TP}+\mathrm{FN}=$ total opportunities for positive calls

$\mathrm{FP}+\mathrm{TN}=$ total opportunities for negative calls

$P D=T P /(T P+F N)$ probability of detection

$\mathrm{PFA}=\mathrm{FP} /(\mathrm{FP}+\mathrm{TN})$ probability of false alarms 
The criteria vary by the amount of box overlap with the buried objects as well as different cutoff values for the parameters being investigated. An example is shown in Figure 6 for the difference in standard deviations with a cutoff value of 1.5 and box overlaps of 0.0, 0.4, 0.6, and 1.0 for the SP object. For reference, the air temperature and rainfall during this period is also shown. Lowering the cutoff value increases the PD while increasing it has the opposite effect. Although not shown, the probability of false alarm (PFA) is higher and the PD is lower for the other objects. The exception to this occurs on 28 August. The standard deviation is unusually large on this day for no obvious reason. Further investigation is needed to deduce whether this is an analysis or possible data error.

Figure 6. Probability of detection and probability of false alarms for the shallow plastic box using a difference in standard deviation cutoff of 1.5. Moving box overlap values are 0.0, 0.4, 0.6, and 1.0. Precipitation and air temperature are provided as points of reference. The dashed line in the upper left figure is at $0^{\circ} \mathrm{C}$.
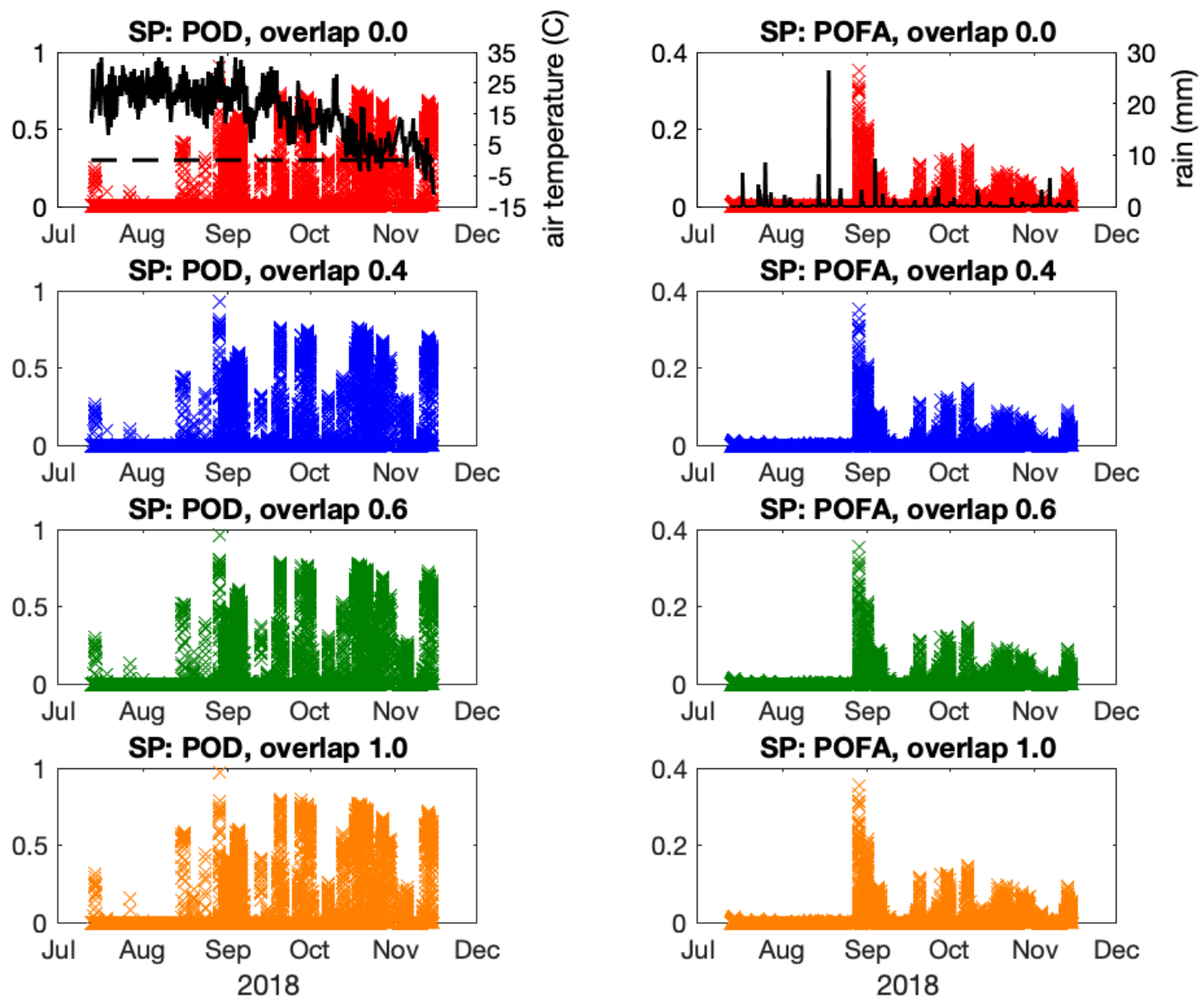


\section{Assessment for Detecting Buried Objects in the Soil Using Multilevel Analyses}

In this section, we investigated buried object detection by employing multilevel modeling statistical analyses using the environmental data and thermal IR imagery collected at the CRREL test plot. The objectives of this subtask were to (1) determine the relational association between environmental inputs and responses and (2) examine the mixed effects on variations between the presence or absence of buried objects for detection.

\subsection{Data for statistical analysis}

The analysis data are aggregated for the period between 15 July and 31 August 2018 given the significant amount of data collected for the entire study period. The data are then separated into four groupings representing the target areas (Figure 7) of buried objects (SP, DP, SM, and DM) and undisturbed locations without buried objects. The first four datasets created are point layouts to compare the targets with the environmental phenomenology variables and are arranged as follows:

- Probe-id-The observations are nested in this variable from the test plot locations. The naming convention is based on individual soil temperature sensor identifiers with each dataset containing the following:

- Group 1 represents the contrast between an SM buried object and undisturbed soil as shown in Figure $7 a$ and includes Probe_id 1-10, 12, 14, and 28 for the undisturbed soil area and Probe_id 19 soil for the SM object location.

- Group 2 denotes a DM buried object together with the undisturbed soil as shown in Figure $7 b$ and includes Probe_id 1-10, 12, 14, and 28 for the undisturbed area and Probe_id 11 for the DM object location.

- Group 3 represents an SP buried object as well as undisturbed area as shown in Figure $7 c$ and includes Probe_id 1-10, 12, 14, and 28 for the undisturbed area and Probe_id 13 for the SP object location. 
- Group 4 represents a DP buried object and the undisturbed area as shown in Figure $7 d$ and includes Probe_id 1-10, 12, 14, and 28 for the undisturbed area and Probe_id 33 for the DP object location.

- Object-Dichotomous variable for contrast areas indicating whether the location has a buried object (1) or none (o).

- AirTemp-Corresponds to an hourly average air temperature in ${ }^{\circ} \mathrm{C}$; each record is the same for the entire plot and every timestamp (the record only varies from one timestamp to the next).

- $R h$-Represents the hourly average relative humidity observations in percent; each record is the same for the entire plot and every timestamp (the record only varies from one timestamp to the next).

- WndSpd-Relates to the hourly average wind speed measurements in $\mathrm{m} / \mathrm{sec}$; each record is the same for the entire plot and every timestamp (the record only varies from one timestamp to the next).

-WndDir-Hourly average wind direction; each measurement is the same for the entire plot and every timestamp (the record only varies from one timestamp to the next).

- $S W$-Corresponds to the hourly average short-wave radiation; each observation is the same for the entire plot and every timestamp (the record only varies from one timestamp to the next).

- $S W R-$ Hourly average short-wave radiation reflectance; each record is the same for the entire plot and every timestamp (the record only varies from one timestamp to the next).

- SoilTemp5-Hourly average soil temperature at $5 \mathrm{~cm}$ at each Probe_id in ${ }^{\circ} \mathrm{C}$; in the case of a buried object location, the soil temperature is measured above the object.

- Timen-Relates to time of day (referring to the $24 \mathrm{hr}$ cycle) the data were collected. Since the data were collected every 15 minutes, the time is aggregated from 1 (midnight) to 24 (midnight); a code of 1 
signifies 2400 to 2445 hours, a code of 2 signifies 0100 to 0145 hours, and so on.

The same timeline (15 July to 31 August 2018) includes thermal images obtained for the entire test plot to compare with the soil and meteorological dataset. A subset of images consisting of a $20 \mathrm{~cm} \times 20$ $\mathrm{cm}$ area of 100 cells representing a $2 \mathrm{~cm} \times 2 \mathrm{~cm}$ resolution is parsed for the locations with buried objects and undisturbed soil (Figure 8 ). The variables for the four datasets used to compare the target areas are arranged as follows:

- Probe-id-The observations are nested in this variable in the test plot. The naming convention is based on the soil temperature sensor identification. Each combination consists of a control plus five $20 \mathrm{~cm} \times 20 \mathrm{~cm}$ areas in the test plot as follows:

- Combination 1 represents the contrast for the SM test plot versus undisturbed area (all the area highlighted in the blue boxes) as shown in Figure 8.

- Combination 2 denotes the comparison between the DM area and undisturbed areas (all the area highlighted in the blue boxes) as shown in Figure 8.

- Combination 3 represents the SP area together with undisturbed areas (all the area highlighted in the blue boxes) as shown in Figure 8.

- Combination 4 represents the DP area along with undisturbed areas (all the area highlighted in the blue boxes) as shown in Figure 8.

- Object-Dichotomous variable for contrast indicating whether the location has either buried object (1) or none (o).

- Cell_id-Relates to each $20 \mathrm{~cm} \times 20 \mathrm{~cm}$ Contrast Area, each consisting of 100 cells representing a $2 \mathrm{~cm} \times 2 \mathrm{~cm}$ resolution. From right to left, the record starts with Cell_id 1 corresponding to the top left cell of the $20 \mathrm{~cm} \times 20 \mathrm{~cm}$ area going 10 cells to the left. 
- Timen-Relates to time of day that the data was collected. A code of 1 signified 2400 to 2445 a.m., code of 2 for 0100 to 0145 , and so on.

- AirTemp-Represents the hourly average air temperature in ${ }^{\circ} \mathrm{C}$; each record is the same for the $20 \mathrm{~cm} \times 20 \mathrm{~cm}$ area, but the observation varies every Timen.

- IR_tir-Corresponds to the hourly average temperature-converted IR radiation values in ${ }^{\circ} \mathrm{C}$ (calculated using the complex method, Equation (8), described in Section 3) for the $2 \mathrm{~cm} \times 2 \mathrm{~cm}$ resolution FLIR image. The hourly $I R \_$tir observations vary for the 100 cells in each $20 \mathrm{~cm} \times 20 \mathrm{~cm}$ area.

- Soil15-Relates to the hourly average soil moisture located at $15 \mathrm{~cm}$ below the ground surface with units in percent by volume. Each record in the 100 cells is the same for the corresponding soil moisture sensor in the $20 \mathrm{~cm} \times 20 \mathrm{~cm}$ area, but the observation varies every Timen. For SM and SP object locations, the moisture sensors were positioned at the vertical midpoint of each buried object.

- SoilTemp5-Represents the hourly average soil temperature at $5 \mathrm{~cm}$ at each Probe_id in ${ }^{\circ} \mathrm{C}$; at the location of a buried object, the soil temperature is measured above the object. Each record in the 100 cells is the same for the corresponding soil temperature sensor in the $20 \mathrm{~cm} \times 20 \mathrm{~cm}$ area, but the observation varies every Timen.

- SoilTemp25-This is an hourly average soil temperature at $25 \mathrm{~cm}$ below ground surface at each Probe_id in ${ }^{\circ} \mathrm{C}$. The soil temperature observations at $25 \mathrm{~cm}$ are measured just below the SM and SP objects and above the DM and DP objects. Each record in the 100 cells is the same for the corresponding soil temperature sensor in the $20 \mathrm{~cm} \times 20 \mathrm{~cm}$ area, but the observation varies every Timen.

Appendix A summarizes the descriptive statistics for the contrast areas the datasets used in the analyses. 
Figure 7. Contrast Area plots (not drawn to scale) show the locations of undisturbed areas (small blue markers) and buried objects (solid and dashed blue and orange boxes with blue small markers in the middle). The four separate datasets for each object include the shaded zones for the (a) SM, (b) DM, (c) SP, and ( $d$ ) DP of the CRREL test plot.

Figure 8. General plot layout (not drawn to scale) for the locations of IR imagery used in the analysis. The boxes denote 100 cells of $2 \mathrm{~cm} \times 2 \mathrm{~cm}$ cells of data above or on SM, SP, DP, and DM and undisturbed locations (blue).

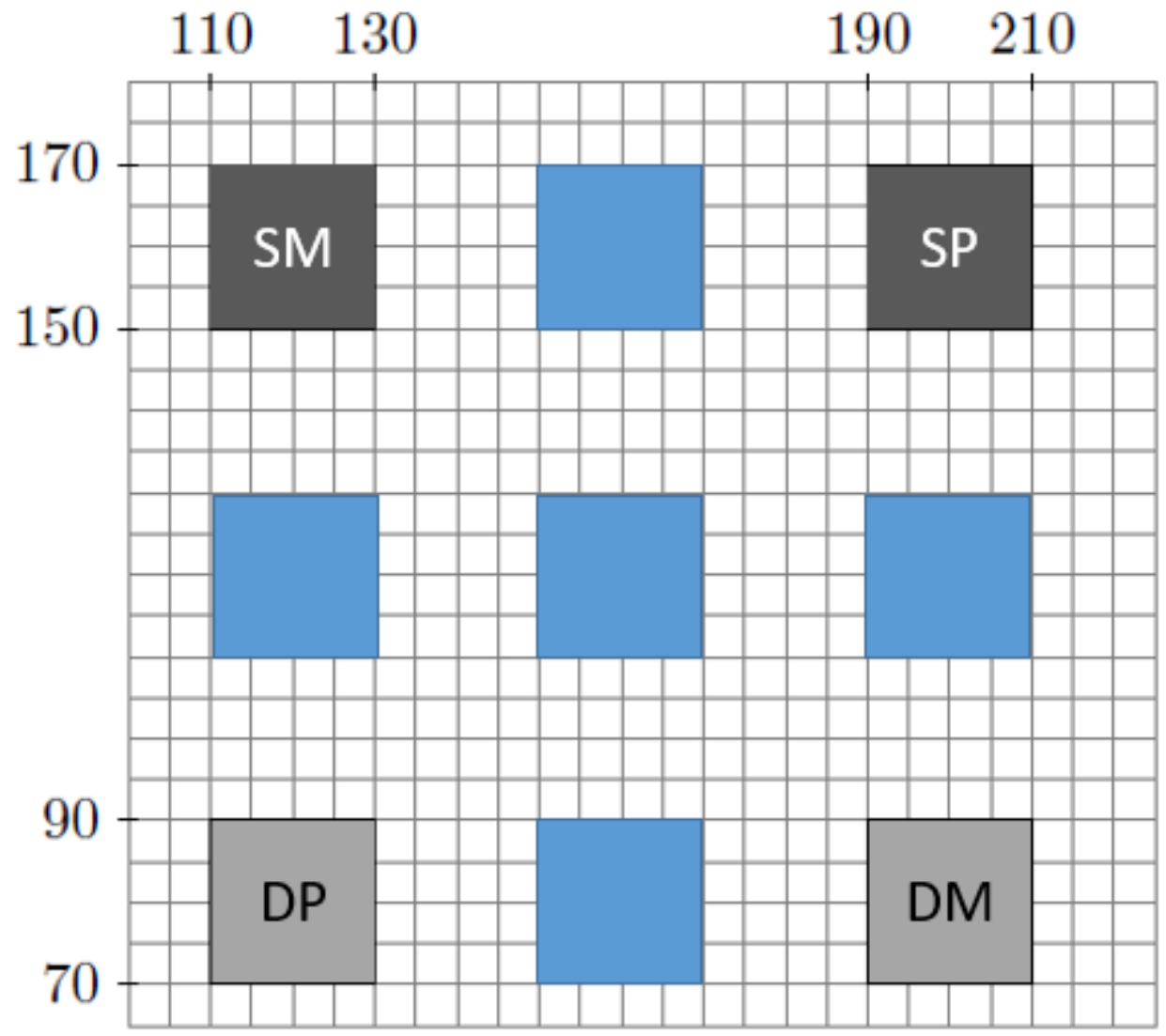

\subsection{Statistical analyses background}

\subsubsection{Logistic regression model}

The basic idea of using the logistic regression model is to describe data in terms of probability and to explain the relationship between one dependent binary variable and one or more nominal, ordinal, interval, or ratio-level independent variables (Hosmer and Lemeshow 2000; McCulloch and Searle 2001; Menard 2002; Snijders and Bosker 1999). The analysis represents a class of fixed effects regression models for several types of variables that may seem disparate (Nelder and Wedderburn 1972; Hedeker 2005). In this case, we want to assess which covariates have the highest probability for detecting the contrast between presence and absence of a buried object. Alternatively, do environmental conditions and soil temperatures have influence on detecting the contrast between having buried objects and undisturbed soil? The regression of an outcome or a dependent variable, $y$, on a predictor or an independent variable, $x$, is a linear function of $x$ that produces the best prediction of $y$. 
In this model, the best prediction means that the result has the smallest mean squared error (Snijders and Bosker 1999). However, logistic regression models do not account for clustering, meaning the data are structured as a single level. Multiple predictor or independent variables can be included in the regression model by accounting for the distribution of the multivariate observations and as long as the model produces the best prediction.

The simple logistical regression model of bivariate distribution is:

$$
y_{i}=\beta_{0}+\beta_{1} x_{i}+\varepsilon_{i}
$$

where

$y_{i}$ is the dependent variable for contrast $i$, and

$x_{i}$ is the independent variable for contrast $i$. The coefficient terms are given by

$$
\beta_{0}=\mathcal{E}(y)-\beta_{1} \mathcal{E}(x), \beta_{1}=\frac{\operatorname{cov}(x, y)}{\operatorname{var}(x)}
$$

where

$$
\begin{aligned}
& \mathcal{E}(x) \text { and } \mathcal{E}(y)=\text { the observation mean or expected value of } x \text { and } y \text {, } \\
& \beta_{0} \text { and } \beta_{1}=\text { intercept and slope of the model, respectively. }
\end{aligned}
$$

In a graphical sense, the intercept is the point where the line in Equation (10) crosses the $y$-axis at $x=0$. The slope, $\beta_{1}$, expresses the relationship between dependent variable $y$ and independent variable $x$. A line with positive slope indicates larger values of $x$ are associated with correspondingly larger values of $y$, while negative slopes mean larger $x$ values are associated with smaller values of $y$. This means regardless of the slope direction, larger values of $\beta 1$ (positive or negative) indicate a stronger linear relationship between dependent and independent variables. The term in Equation (10), $\varepsilon$, is the residual or error component.

\subsubsection{Multilevel models}

A multilevel model approach allows an analysis with nested or multilevel data (Affleck et al. 2019; Ene et al. 2015; Heck and Thomas 2000; Hox 2002; Klein and Kozlowski 2000; Raudenbush and Bryk 2002; Snijders 
and Bosker 1999). Also, multilevel models incorporate the randomness and nonlinearity of outcomes in the analysis of the nested data. These models have also been referred to as hierarchical linear models, fixed effects models, mixed models, random coefficient models, and covariance component models (Snijders and Bosker 1999; Hox 2002). These statistical models are used to analyze nested sources of variability in hierarchical data, taking into account the variability associated with each level of the hierarchy (Ene et al. 2015; Garson 2012; Heck and Thomas 2000; Hox 2002; Klein and Kozlowski 2000; Raudenbush and Bryk 2002; Snijders and Bosker 1999). These models assume all observations are independent of each other and are appropriate for analysis of several types of correlated data structures such as nested or longitudinal data. In nested designs, subjects or measurements are observed clustered within larger units (e.g., schools, hospitals, neighborhoods, workplaces, and so on). In longitudinal designs with temporal variables, repeated measurements are nested within subjects or test locations. The level-1 observations (variables or repeated measurements) are nested within the higher level-2 observations (e.g., clusters within the respective test plots).

A multilevel model augments the logistic regression model by estimating the intercept of the level-1 dependent variable as an effect of the level-2 grouping variable and possibly other level-1 or level-2 (or higher) covariates. The association of nonlinearity of outcomes is modeled by computing the probability or likelihood estimation of a response. The simplified combination of level-1 and level-2 model yields a clusterspecific model of response as follows:

$$
y_{i j}=\beta_{0 j}+\beta_{1 j} x_{i j}+\varepsilon_{i j}
$$

where $y_{i j}$ is the dependent variable for contrast $i$, and $x_{i}$ is the independent variable for contrast $i$. In our model, $i$ denotes the level-2 units (e.g., Probe_id) and $j$ denotes the level-1 units (e.g., nested observations). Assume there are $i=1, \ldots, N$ for Probe_id test locations (level-2 units) and $j=1, \ldots, n_{i}$ for repeated observations (level-1 units) that are nested within each test location. The intercept $\beta_{0 j}$ accounts for the mean likelihood of a response in Probe_id, $j$ controlling the effects in the model. The term $\beta_{1 j}$ is the slope associated with this predictor, $x_{i j}$. The term $\varepsilon_{i j}$ represents a residual error for the effect associated within the Probe_id variations. 


\subsubsection{Analytical steps}

The analytical steps in the statistical analyses included the overarching question of the best way to determine the relational association between atmospheric input and response for detecting the contrast between presence and absence of a buried object in the ground. To address the overarching question, the statistical analyses are separated into two approaches: (1) using an unclustered structure with logistic regression model to identify which covariates (environmental properties, soil conditions, and near-surface soil temperatures) have improved probability for detecting the contrast between presence/absence of a buried object (the multilevel model primarily assesses the overall relationship of covariates for detecting the contrast) and (2) incorporating the clustering structure of the data with nonlinearity and randomness of outcomes with a multilevel model, which utilizes the following sequences:

a) A dichotomous procedure to identify which covariates can detect the contrast areas

b) Using a continuous outcome with the generalization that the data are permitted to exhibit correlation and nonconstant variability to determine the overall probability of detecting the contrast areas or which contrast areas are significant. How do contrast area vary by the time of day? What time of the day has the highest unit change (probability) for discriminating the contrast areas?

The model-building processes are listed in Table 4, which describes what effects are included in each of the models and the information about what output the various models provide. Each dependent variable is divided into four contrast areas using the SAS software (version 9.4, SAS Institute 2017) with three types of statistical procedures: the PROC LOGISTICS procedure without clustering effects, PROC GLIMMIX, and PROC MIXED.

The PROC LOGISTICS procedure is a logistic regression analysis that is often used to investigate the relationship between discrete responses (such as binary, ordinal and nominal) and a set of explanatory variables responses without the clustering effects (https://documentation.sas.com/doc/en/pgmsascdc/9.4_3.4/statug/statug_logistic_overview.htm). The PROC GLIMMIX procedure is used to investigate the relationship 
between discrete responses and a set of explanatory variables such as binary or dichotomous, ordinal and nominal responses with clustering effects (https://support.sas.com/rnd/app/stat/procedures/glimmix.html). The PROC MIXED is used to investigate the relationship between a continuous variable and a set of explanatory variables with clustering effects (https://documentation.sas.com/doc/en/pgmsascdc/9.4_3.3/statug/statug_mixed_overview.htm).

The analytical steps A1 and A2 (Table 4) used Object as a dependent or outcome variable with a logistic regression model. The goal for this step is to determine the probability of differentiating the presence of buried objects from undisturbed areas and the relationship between Object and other covariates or predictors (e.g., soil temperatures and changes in atmospheric conditions). The second procedure applied the multilevel model with Object as a level 1-dependent or outcome variable for analytical step A3 (Table 4) with a binary solution function in the PROC GLIMMIX procedure (SAS Institute 2017). The analytical step A3 model consisted of predictors in level 1 to determine the relationships between the level 1 outcome and the level 2 effects (in this case, using the Probe-id). The third approach used the multilevel model with continuous variables as dependent outcome measurements such as SoilTemp5 SoilTemp25, IR_tir and Soil15. In these analytical steps (B1, B2, B3, C and D), the procedure used the SAS PROC MIXED with Object variable as predictors in level 1 to determine the relationships between the level 1 outcome and the level 2 effects (in this case, using the Probe-id). In each analytical step for B1, B2, B3, C and D, 96 individual models are created for all four contrast areas.

Table 4. Summary statistical models used in the analyses.

\begin{tabular}{|l|l|l|}
\hline $\begin{array}{c}\text { Outcome or } \\
\text { Dependent } \\
\text { Variable }\end{array}$ & $\begin{array}{c}\text { Analytical } \\
\text { Steps }\end{array}$ & \multicolumn{1}{c|}{ Model Description } \\
\hline \multirow{5}{*}{ Object } & A1 & $\begin{array}{l}\text { Logistic regression model (without clustering } \\
\text { effects) for individual contrast using the point } \\
\text { layout datasets of the soil temperature and } \\
\text { environmental variables }\end{array}$ \\
\cline { 2 - 3 } & A2 & $\begin{array}{l}\text { Logistic regression model (without clustering } \\
\text { effects) for individual contrast using the 20 } \\
\text { cm } \times 20 \text { cm area with IR, soil temperature, } \\
\text { and soil moisture }\end{array}$ \\
\cline { 2 - 3 } & A3 & $\begin{array}{l}\text { Multilevel model (GLIMMIX procedure, } \\
\text { LAPLACE and binomial function) for } \\
\text { individual contrast using the point layout } \\
\text { datasets of the soil temperature and } \\
\text { environmental variables as level 1 predictors } \\
\text { and nested in Probe-id as level 2 variable }\end{array}$ \\
\hline
\end{tabular}




\begin{tabular}{|c|c|c|}
\hline $\begin{array}{c}\text { Outcome or } \\
\text { Dependent } \\
\text { Variable }\end{array}$ & $\begin{array}{c}\text { Analytical } \\
\text { Steps }\end{array}$ & Model Description \\
\hline SoilTemp5 & B1 & $\begin{array}{l}\text { Multilevel model (MIXED procedure) for } \\
\text { individual contrast with Object as level } 1 \\
\text { predictor and nested in Probe-id as level } 2 \\
\text { variable using the point layout datasets. } 96 \\
\text { individual models are run for all four } \\
\text { contrasts. }\end{array}$ \\
\hline $\begin{array}{l}\text { SoilTemp5 Time1, } \\
\ldots \\
\text { SoilTemp5 Time24 }\end{array}$ & B2 & $\begin{array}{l}\text { Multilevel model (MIXED procedure) for } \\
\text { individual hourly contrast with Object as level } \\
1 \text { predictor and nested in Probe-id as level } 2 \\
\text { variable using the point layout datasets. } 96 \\
\text { individual models are run for all four } \\
\text { contrasts. }\end{array}$ \\
\hline \begin{tabular}{|l} 
SoilTemp25 \\
Time1, ... \\
SoilTemp25 \\
Time24
\end{tabular} & B3 & $\begin{array}{l}\text { Multilevel model (MIXED procedure) for } \\
\text { individual hourly contrast with Object as level } \\
1 \text { predictor and nested in Probe-id as level } 2 \\
\text { variable using the point layout datasets }\end{array}$ \\
\hline $\begin{array}{l}\text { IR_tir Time1, } \\
\cdots \\
\text { IR_tir Time24 }\end{array}$ & C & $\begin{array}{l}\text { Multilevel model (MIXED procedure) for } \\
\text { individual hourly contrast with Object as level } \\
1 \text { predictor and nested in Probe-id as level } 2 \\
\text { variable using the } 20 \mathrm{~cm} \times 20 \mathrm{~cm} \text { area } \\
\text { datasets. } 96 \text { individual models are run for all } \\
\text { four contrasts. }\end{array}$ \\
\hline $\begin{array}{l}\text { Soil15 Time1, } \\
\ldots \\
\text { Soil15 Time24 }\end{array}$ & D & $\begin{array}{l}\text { Multilevel model (MIXED procedure) for } \\
\text { individual hourly contrast with Object as level } \\
1 \text { predictor and nested in Probe-id as level } 2 \\
\text { variable. } 96 \text { individual models are run for all } \\
\text { four contrasts. }\end{array}$ \\
\hline
\end{tabular}

\subsection{Statistical analyses results}

\subsubsection{Model A: Object as a dependent variable}

The parameter estimates for logistic regression model using Object as a dependent variable from analytical step A1 are shown in Table 5 with the point layout datasets and in Table 6 from analytical step A2 using the 20 $\mathrm{cm} \times 20 \mathrm{~cm}$ area datasets. The logistic regression model is used without clustering effects, and the results from the logistic regression model include the following:

- Group 1 (SM buried object versus undisturbed soil): The intercept for Object has statistical significance (Table 5). However, the probabilities of the regressors have no statistical significance on detecting the contrast area for the SM and undisturbed areas. The beta or log odds estimated using logistic regression model are less 
than or close to zero, which means these regressors have no effect on the variable Object.

Using the $20 \mathrm{~cm} \times 20 \mathrm{~cm}$ area dataset, the variable Object (Table 6) has statistical significance $(p<0.01)$ for its intercept and is overall statistically related $(p<0.01)$ to hourly average surface temperature (IR_tir), soil temperature at $5 \mathrm{~cm}$ bgs (SoilTemp5), and soil moisture at $15 \mathrm{~cm}$ bgs (Soil15). The overall odds for detecting the change in the temperature response between the undisturbed and SM object area decrease with decreasing surface soil temperature and increase with increasing surface soil temperature. In this case the surface soil temperature changes approximately $-0.93^{\circ} \mathrm{C}$ as measured by thermal IR (IR_tir). Soil moisture is also a significant contributor to the contrast difference between undisturbed soil and SM metal object by approximately $35.75 \%$ volume. It is important to note the soil moisture sensor is positioned adjacent ( $5 \mathrm{~cm}$ away) to the $\mathrm{SM}$ object at its vertical midpoint.

- Group 2 (DM versus undisturbed soil): The intercept for Object (Table 5) has statistical significance $(p<0.01)$. The probabilities of hourly average air temperature (Airtemp) and soil temperature at 5 $\mathrm{cm}$ (SoilTemp5) also have statistical significance for detecting the contrast between the DM object and undisturbed soil. The remaining environmental regressors have no effect for estimating the difference between undisturbed soil and the DM object.

The parameter estimates using the $20 \mathrm{~cm} \times 20 \mathrm{~cm}$ area dataset showed the variable Object (Table 6) has statistical significance ( $p<$ o.01) for its intercept and is statistically related $(p<0.01)$ to hourly average surface temperature (IR_tir), soil temperature (SoilTemp5), and soil moisture (Soil15). The odds for a change in response to soil temperature from the undisturbed to SM object area increases with increasing surface soil temperature and decreases with decreasing surface soil temperature. Specifically, this means the soil above the SM object as measured by the thermal IR (IR_tir) is about $0.57^{\circ} \mathrm{C}$ warmer than the undisturbed soil. Soil moisture also contributes to a difference between buried object and no object. The change from no object DM object area is approximately $78.44 \%$ by volume of soil moisture, indicating the 
soils above the DM are wetter than the background soils. It is important to note the soil moisture sensor is positioned $10 \mathrm{~cm}$ above the deep metal object. This suggests water from precipitation events migrates into the soil and then pools on top of the DM object.

- Group 3 (SP versus undisturbed soil): The intercept for Object as a dependent variable has statistical significance $(p<0.01)$. The probabilities of hourly average air temperature (Airtemp), soil temperature at $5 \mathrm{~cm}$ bgs (SoilTemp5), time of day (Timen), relative humidity $(R h)$, and wind speed (WindSpd) also have statistical significance for detecting the difference between the SP object area and undisturbed soil. The remaining environmental regressors have no effect on the contrast (variable Object).

Similarly, the variable Object (Table 6) has statistical significance ( $p$ $<0.01$ ) based on the logistic regression model using the 20 by 20 $\mathrm{cm}$ area dataset. The hourly average surface soil temperature (IR_tir), soil temperature at $5 \mathrm{~cm}$ bgs (SoilTemp5), and soil moisture at $15 \mathrm{~cm}$ bgs (Soil15) are also statistically significant to the difference between SP area and undisturbed soil. This means the odds for change between undisturbed soil and the SP object area increases with increasing surface soil temperature as measured with thermal IR (IR_tir) by approximately $1^{\circ} \mathrm{C}$. Soil moisture is also a significant contributor to the contrast with a change between undisturbed soil and a SP object area by approximately $17 \%$ by volume. It is important to note the soil moisture sensor is positioned adjacent ( $5 \mathrm{~cm}$, away) to the $\mathrm{SP}$ object at its vertical midpoint.

- Group 4 (DP versus undisturbed soil): The intercept for Object as a dependent variable has statistical significance $(p<0.01)$. The probabilities of hourly average air temperature (Airtemp), soil temperature at $5 \mathrm{~cm}$ (SoilTemp5), and relative humidity $(R h)$ have a statistically significant influence on the ability of seeing the contrast between the DP object area and undisturbed soil area. The remaining environmental regressors have no effect on the contrast (variable Object). 
Likewise, the parameter estimates using the $20 \mathrm{~cm} \times 20 \mathrm{~cm}$ area dataset showed that the variable Object (Table 6) has statistical significance $(p<0.01)$. The hourly average surface soil temperature as measured with thermal IR (IR_tir), soil temperature at $5 \mathrm{~cm}$ (SoilTemp5), and soil moisture (Soil15) have a statistically significant impact on the contrast area. This means that the probability for a detectable change from an undisturbed area to a DP object area increases with increasing temperature. The thermal IR (IR_tir) signature for the DOP object area is approximately $0.48^{\circ} \mathrm{C}$ warmer than the undisturbed area. Soil moisture is also a significant contributor to the contrast between undisturbed soil and the DM object area by approximately $75 \%$ volume of soil moisture. It is important to note the soil moisture sensor is positioned $10 \mathrm{~cm}$ above the deep metal object.

Table 5. Model A1 output of probability estimates and standard errors (values in parenthesis) from logistic regression model with intercept of Object as a dependent variable using the point layout datasets of the hourly average soil temperature and environmental variables.

\begin{tabular}{|l|r|r|r|r|}
\hline \multicolumn{1}{|c|}{ Fixed Effects } & \multicolumn{1}{c|}{ SM } & \multicolumn{1}{c|}{ DM } & \multicolumn{1}{c|}{ SP } & \multicolumn{1}{c|}{ DP } \\
\hline Intercept (Object) & $-2.505(0.55)^{\oplus}$ & $-3.56(0.56)^{\oplus}$ & $-5.24(0.52)^{\oplus}$ & $-4.488(0.561)^{\oplus}$ \\
\hline Airtemp & $-0.002(0.017)$ & $-0.03(0.017)^{\oplus}$ & $-0.091(0.015)^{\oplus}$ & $-0.062(0.016)^{\oplus}$ \\
\hline SoilTemp5 & $-0.003(0.019)$ & $0.049(0.018)^{\oplus}$ & $0.132(0.014)^{\oplus}$ & $0.093(0.018)^{\oplus}$ \\
\hline Timen & $0.0002(0.005)$ & $-0.003(0.005)$ & $-0.008(0.005)^{\dagger}$ & $-0.0059(0.005)$ \\
\hline Rh & $-0.0003(0.004)$ & $0.005(0.004)$ & $0.016(0.004)^{\oplus}$ & $0.0102(0.004)^{\oplus}$ \\
\hline SW & $-0.00001(0.0006)$ & $-0.0003(0.0006)$ & $0.0006(0.0006)$ & $0.0005(0.0006)$ \\
\hline SWR & $0.0001(0.004)$ & $-0.00195(0.004)$ & $-0.0054(0.004)$ & $-0.0039(0.004)$ \\
\hline Rain & $0.0004(0.0785)$ & $-0.0067(0.0795)$ & $-0.0202(0.0819)$ & $-0.0125(0.0804)$ \\
\hline WndSpd & $-0.003(0.073)$ & $0.046(0.073)$ & $0.1263(0.0725)^{\dagger}$ & $0.0860(0.0726)$ \\
\hline WndDir & $8.9 \mathrm{e}-6(0.0006)$ & $1.5 \mathrm{e}-4(0.0006)$ & $0.0004(0.0006)$ & $0.0003(0.0006)$ \\
\hline Model fit, AIC & 8302.07 & 8302.07 & 8124.66 & 8302.07 \\
\hline Model fit, -2 Log L & 8300.07 & 8300.07 & 8122.66 & 8300.07 \\
\hline
\end{tabular}

${ }^{\oplus} p<.01 ;{ }^{\star} p<.05 ;{ }^{\dagger} p<.10$ 
Table 6. Model A2 results of probability estimates and standard errors (values in parenthesis) from logistic regression modeling with intercept of Object as a dependent variable using the $20 \mathrm{~cm} \times 20 \mathrm{~cm}$ area with the hourly average IR temperature, soil temperature, and soil moisture.

\begin{tabular}{|l|r|r|r|r|}
\hline \multirow{2}{*}{ Fixed Effects } & \multicolumn{5}{|c|}{ Groups of Contrast } \\
\cline { 2 - 5 } & \multicolumn{1}{|c|}{ SM } & \multicolumn{1}{c|}{ DM } & \multicolumn{1}{c|}{ SP } & \multicolumn{1}{c|}{ DP } \\
\hline Intercept (Object) & $-87.41(0.91)^{\oplus}$ & $-15.7(1.1)^{\oplus}$ & $-74.26(0.74)^{\oplus}$ & $-7.15(1.19)^{\oplus}$ \\
\hline AirTemp & $-0.04(0)^{\oplus}$ & $0.43(0)^{\oplus}$ & $0.14(0)^{\oplus}$ & $0.42(0)^{\oplus}$ \\
\hline IR_tir & $-0.93(0.01)^{\oplus}$ & $-0.57(0.01)^{\oplus}$ & $-1.02(0.01)^{\oplus}$ & $-0.48(0.02)^{\oplus}$ \\
\hline Soil15 & $34.75(0.14)^{\oplus}$ & $-78.44(0.22)^{\oplus}$ & $-17.42(0.17)^{\oplus}$ & $-75.23(0.21)^{\oplus}$ \\
\hline Soiltemp5 & $0.04(0)^{\oplus}$ & $-0.2(0)^{\oplus}$ & $0.01(0)^{\oplus}$ & $-0.16(0)^{\oplus}$ \\
\hline Model fit, AIC & & & 622857.82 \\
\hline Model fit, -2 Log L & \multicolumn{5}{|c}{} \\
\hline$\oplus p<0.01 ; * p<0.05 ; p<0.10$ & & & 622855.82 \\
\hline
\end{tabular}

${ }^{\oplus} p<0.01 ;{ }^{*} p<0.05 ;{ }^{\dagger} p<0.10$

Taking the summer datasets as whole for each group of contrasts, results from clustering effects using GLIMMIX, binary solution function with Object as a dependent variable, indicate none of the environmental variables are related (Table 7). When the Probe-id is added (level 2) for clustering effects, the dependent variable (Object) at level 1 having values of $\mathrm{o}$ and 1 does not vary by subsurface thermistor location. The estimates of the random effects are all the same. In other words, the outcome is the same within subsurface thermistor location at a summer observation. Thus modeling the Object variable as an outcome $(y)$ variable is not an appropriate approach for this model in incorporating the clustering effects of level 2 (Probe-id) to use for detecting the contrast between the presence and absence of the objects. Using a continuous variable from in situ soil measurements as an outcome, as opposed to the Object variable, will provide the distinctive results for detecting the target (Section 4.3.2). 
Table 7. Model A3 output of probability estimates and standard errors (values in parenthesis) from multilevel model procedures with intercept of Object as a dependent variable using GLIMMIX procedure and LAPLACE function. These estimates used the point layout datasets of the hourly average soil temperature and environmental variables.

\begin{tabular}{|l|r|r|r|r|}
\hline \multicolumn{1}{|c|}{ Fixed Effects } & \multicolumn{1}{c|}{ SM } & \multicolumn{1}{c|}{ DM } & \multicolumn{1}{c|}{ SP } & \multicolumn{1}{c|}{ DP } \\
\hline Intercept (Object) & $-4.53(93.89)$ & $-5.52(93.38)$ & $-7.15(85.96)$ & $-6.457(94.067)$ \\
\hline Airtemp & $0.223(3.006)$ & $0.176(2.814)$ & $-0.020(2.495)$ & $0.173(2.858)$ \\
\hline SoilTemp & $-0.4303(2.997)$ & $-0.352(2.684)$ & $-0.130(1.752)$ & $-0.348(2.798)$ \\
\hline Timen & $0.0059(1.275)$ & $0.0043(1.225)$ & $-0.0403(1.242)$ & $0.008(1.166)$ \\
\hline Rh & $-0.117(0.662)$ & $-0.117(0.662)$ & $-0.112(0.638)$ & $-0.108(0.660)$ \\
\hline SW & $-0.0031(0.095)$ & $-0.0032(0.097)$ & $-0.00105(0.092)$ & $-0.003(0.100)$ \\
\hline SWR & $0.0257(0.62)$ & $0.0237(0.625)$ & $0.0059(0.6)$ & $0.0224(0.65)$ \\
\hline Rain & $-0.2014(2.854)$ & $-0.166(2.947)$ & $-0.0285(4.796)$ & $-0.218(2.643)$ \\
\hline WndSpd & $-2.667(13.451)$ & $-2.798(13.765)$ & $-2.5764(12.6850)$ & $-2.492(13.483)$ \\
\hline WndDir & $-0.0077(0.1365)$ & $-0.0079(0.1367)$ & $-0.0056(0.1345)$ & $-0.0065(0.133)$ \\
\hline Model fit, AlC & 25.80 & 25.80 & 25.80 & 25.79 \\
\hline Model fit, -2 Log L & 3.80 & 3.80 & 3.80 & 3.79 \\
\hline \multicolumn{1}{|r|}{$\oplus_{p}<.01 ; * p<.05 ; \dagger p<.10$} & & & \\
\end{tabular}

${ }^{\oplus} p<.01 ;{ }^{*} p<.05 ; \uparrow p<.10$

\subsubsection{Model B: Soil temperature as outcome}

The soil temperature measurements are slightly different within probes and vary considerably with time. Having the soil temperature measurements (SoilTemp5 and SoilTemp25) individually modeled as an outcome, the Object variable is assigned as the predictor for detecting the contrast between the presence of objects and undisturbed soil. The multilevel model using the MIXED procedure is used given that soil temperature is a continuous variable and the model is nested at level 2 with a Probe-id variable.

Table 8 provides the parameter estimate summary for the timeline between 15 July and 31 August 2018 using the multilevel (MIXED) models with clustering effects, a level 2 (Probe-id), and the level 1 outcome of soil temperature (SoilTemp5). The results indicate the hourly average soil temperatures at $5 \mathrm{~cm}$ (SoilTemp5) for all the contrasting groups are statistically significant $(p<0.01)$ with estimated intercept ranging between 19.7 and $19.9^{\circ} \mathrm{C}$. The soil temperatures at $5 \mathrm{~cm}$ (SoilTemp5) are statistically associated with air temperature (Airtemp), time of day (Timen), relative humidity $(R h)$, short wave $(S W)$, short-wave reflectance 
$(S W R$ ), and wind speed (WindSpd). The relationship with various slope values is naturally related to the temperature influence and energy balance or the surface/atmosphere radiation and absorption exchange. Generally, there is a statistical significance and positive relationship between soil temperature and Object variable particularly with plastic objects. On average, having a shallow plastic object buried in the ground increases (slope) soil temperature by $0.56^{\circ} \mathrm{C}$ and influences other variables in the model. Similarly, a DP buried object increases slope of the soil temperature by $0.26^{\circ} \mathrm{C}$.

Table 8. Model B1 results of probability estimates and standard errors (values in parenthesis) from multilevel model applying the PROC MIXED procedures for all contrasts with intercept of SoilTemp5 as a dependent variable. These estimates used the point layout datasets of the hourly average soil temperature and environmental variables.

\begin{tabular}{|l|r|r|r|r|}
\hline \multicolumn{1}{|c|}{ Fixed Effects } & \multicolumn{1}{c|}{ SM } & \multicolumn{1}{c|}{ DM } & \multicolumn{1}{c|}{ SP } & \multicolumn{1}{c|}{ DP } \\
\hline Intercept (SoilTemp5) & $19.775(0.175) \oplus$ & $19.905(0.177) \oplus$ & $19.67(0.204) \oplus$ & $19.951(0.177) \oplus$ \\
\hline Object & $-0.008(0.118)$ & $0.1354(0.118)$ & $0.542(0.118) \oplus$ & $0.262(0.118)^{\star}$ \\
\hline Airtemp & $0.696(0.005) \oplus$ & $0.672(0.005) \oplus$ & $0.696(0.006) \oplus$ & $0.670(0.005) \oplus$ \\
\hline Timen & $0.061(0.002) \oplus$ & $0.062(0.002) \oplus$ & $0.063(0.002) \oplus$ & $0.062(0.002) \oplus$ \\
\hline Rh & $-0.102(0.001) \oplus$ & $-0.103(0.001) \oplus$ & $-0.107(0.002) \oplus$ & $-0.104(0.001) \oplus$ \\
\hline SW & $-0.004(0.0002) \oplus$ & $-0.004(0.0002) \oplus$ & $-0.004(0.0003) \oplus$ & $-0.005(0.0002) \oplus$ \\
\hline SWR & $0.037(0.002) \oplus$ & $0.037(0.002) \oplus$ & $0.035(0.002) \oplus$ & $0.037(0.002) \oplus$ \\
\hline Rain & $0.133(0.033) \oplus$ & $0.134(0.033) \oplus$ & $0.144(0.038) \oplus$ & $0.131(0.033) \oplus$ \\
\hline WndSpd & $-0.945(0.03) \oplus$ & $-0.946(0.03) \oplus$ & $-0.972(0.035) \oplus$ & $-0.948(0.03) \oplus$ \\
\hline WndDir & $-0.003(0.0003) \oplus$ & $-0.003(0.0003) \oplus$ & $-0.003(0.0003) \oplus$ & $-0.003(0.0003) \oplus$ \\
\hline Model fit, AIC & 61507.2 & 61841.8 & 66511.5 & 61825.7 \\
\hline Model fit, -2 Log L & 61503.2 & 61837.8 & 66507.5 & 61821.7 \\
\hline
\end{tabular}

${ }^{\oplus} p<.01 ;{ }^{\star} p<.05 ;{ }^{\dagger} p<.10$

Given soil temperature at $5 \mathrm{~cm}$ (SoilTemp5) for all contrasts are statistically significant, this raises the question of what time of day has the highest change (probability) for estimating the contrasts or what is the best time for detecting buried objects? Using nested observations at level 2 with Probe-id variable, the multilevel model (MIXED procedure) generated individual hourly contrasts with SoilTemp5 as an outcome and Object as level-1 predictor using the point layout datasets. The individual hourly contrast model for time of day $(1,2, \ldots 24)$ estimates the probability of the intercepts for soil temperatures at $5 \mathrm{~cm}$ and slopes of the 
relationship between the hourly soil temperature intercepts and slopes of Object from o (no object) to 1 (with object) for the corresponding contrasts (Table 9). For all contrasts, the estimated intercepts for SoilTemp 5 have statistical significance $(p<.01)$ and are the same within the specific time of the day.

For example, the intercept at time code 2 from 0100 to 0145 is $22.6^{\circ} \mathrm{C}$, which is the mean soil temperature probability distribution and controls for all effects in the model for that particular time of the day. The estimated intercepts for soil temperatures at $5 \mathrm{~cm}$ diurnally fluctuates from $21.1^{\circ} \mathrm{C}$ at 0600 to $31.7^{\circ} \mathrm{C}$ at 1500 with a $10.6^{\circ} \mathrm{C}$ increase due to heat gain. The slope estimates of Object from o (no object) to 1 (with object) correspond to the change in temperature and differ for each contrast in relation to undisturbed areas. The slope estimates have statistical significance primarily for the SP and SM contrast but not for the DM and DP contrasts. A negative slope means the temperature is decreasing during that time of the day above the objects, while the positive change or slope means there is warming relative to the intercept in the presence of buried objects. For example, the soil temperature above the SP object from 0100 to 0145 is approximately $-2.6^{\circ} \mathrm{C}$ relative to the intercept value of $22.6^{\circ} \mathrm{C}$ (about $20.6^{\circ} \mathrm{C}$ above the $\mathrm{SP}$ object) and about $-0.5^{\circ} \mathrm{C}$ relative to the intercept value of $22.6^{\circ} \mathrm{C}$ for the SM object (about $22.1^{\circ} \mathrm{C}$ above the SM object). At this time the ground is slightly warmer above the SM object than the SP object. At time code 10 or around 0900, the estimated slopes of the Object for the SP and SM objects are almost equal; this means that soil temperatures above the SP and SM are almost identical. However, from 1300 to 1345 , the soil temperatures above the SP and SM are estimated to be 5.5 and $1.1^{\circ} \mathrm{C}$, respectively relative to the intercept of $30.9^{\circ} \mathrm{C}$ (about $36.4^{\circ} \mathrm{C}$ and $32^{\circ} \mathrm{C}$ above the SP and SM objects, respectively). This means that at 1300 the ground is $4^{\circ} \mathrm{C}$ warmer above the SP object than the SM object. 
Table 9. Model B2 output of probability estimates and standard errors (values in parenthesis) from 96 multilevel models of applying the PROC MIXED procedures aggregated based on time of day contrasts with intercept of soil temperature at $5 \mathrm{~cm}$ (SoilTemp) as a dependent variable.

\begin{tabular}{|c|c|c|c|c|c|}
\hline \multirow[t]{3}{*}{ Time of day } & \multirow{3}{*}{$\begin{array}{c}\text { Intercept } \\
\text { (SoilTemp5) }\end{array}$} & \multicolumn{4}{|c|}{ Contrast (Objects versus without) } \\
\hline & & SM & DM & SP & DP \\
\hline & & \multicolumn{4}{|c|}{ Slope of Object } \\
\hline $1(12-12: 45 a m)$ & $23.12(0.08) \oplus$ & $-0.598(0.3)^{*}$ & $-0.20(0.30)$ & $-2.406(0.309) \oplus$ & $0.25(0.30)$ \\
\hline $2(1-1: 45 a m)$ & $22.6(0.08) \oplus$ & $-0.522(0.301) \dagger$ & $-0.27(0.30)$ & $-2.558(0.31) \oplus$ & $0.21(0.30)$ \\
\hline $3(2-2: 45 a m)$ & $22.17(0.08) \oplus$ & $-0.447(0.303)$ & $-0.296(0.31)$ & $-2.625(0.314) \oplus$ & $0.17(0.31)$ \\
\hline 4 (3-3:45am) & $21.82(0.08) \oplus$ & $-0.389(0.306)$ & $-0.33(0.31)$ & $-2.628(0.317) \oplus$ & $0.12(0.31)$ \\
\hline 5 (4-4:45am) & $21.5(0.08) \oplus$ & $-0.339(0.312)$ & $-0.35(0.31)$ & $-2.614(0.323) \oplus$ & $0.08(0.31)$ \\
\hline $6(5-5: 45 a m)$ & $21.24(0.09) \oplus$ & $-0.298(0.319)$ & $-0.36(0.32)$ & $-2.574(0.33) \oplus$ & $0.04(0.32)$ \\
\hline 7 (6-6:45am) & $21.1(0.09) \oplus$ & $-0.226(0.323)$ & $-0.36(0.33)$ & $-2.465(0.334) \oplus$ & $0.005(0.32)$ \\
\hline $8(7-7: 45 a m)$ & $21.43(0.09) \oplus$ & $-0.011(0.321)$ & $-0.299(0.32)$ & $-1.965(0.33) \oplus$ & $-0.02(0.32)$ \\
\hline 9 (8-8:45am) & $22.39(0.09) \oplus$ & $0.319(0.312)$ & $-0.16(0.31)$ & $-0.926(0.319) \oplus$ & $-0.01(0.31)$ \\
\hline 10 (9-9:45am) & $23.86(0.09) \oplus$ & $0.679(0.32) *$ & .32) & $0.512(0.33)$ & $0.027(0.32)$ \\
\hline 11 (10-10:45am) & $25.76(0.1) \oplus$ & $1.04(0.36) \oplus$ & $0.22(0.35)$ & $2.163(0.38) \oplus$ & $0.098(0.34)$ \\
\hline 12 (11-11:45am) & $27.76(0.12) \oplus$ & $1.27(0.43) \oplus$ & $0.46(0.43)$ & $3.76(0.46) \oplus$ & $0.208(0.43)$ \\
\hline $13(12-12: 45 p m)$ & $29.55(0.13) \oplus$ & $1.28(0.49) \oplus$ & $0.61(0.49)$ & $4.909(0.52) \oplus$ & $0.298(0.49)$ \\
\hline $14(1-1: 45 p m)$ & $30.88(0.15) \oplus$ & $1.14(0.55)^{*}$ & $0.69(0.55)$ & $5.508(0.584) \oplus$ & $0.365(0.55)$ \\
\hline $15(2-2: 45 p m)$ & $31.56(0.16) \oplus$ & $0.827(0.61)$ & $0.71(0.61)$ & $5.509(0.646) \oplus$ & $0.414(0.61)$ \\
\hline $16(3-3: 45 p m)$ & $31.67(0.17) \oplus$ & $0.477(0.635)$ & $0.75(0.64)$ & $5.127(0.673) \oplus$ & $0.475(0.64)$ \\
\hline $17(4-4: 45 p m)$ & $31.35(0.17) \oplus$ & $0.166(0.649)$ & $0.73(0.65)$ & $4.452(0.686) \oplus$ & $0.522(0.65)$ \\
\hline $18(5-5: 45 p m)$ & $30.62(0.17) \oplus$ & $-0.128(0.627)$ & $0.66(0.63)$ & $3.564(0.662) \oplus$ & $0.565(0.63)$ \\
\hline 19 (6-6:45pm) & $29.54(0.15) \oplus$ & $-0.436(0.57)$ & $0.54(0.58)$ & $2.456(0.599) \oplus$ & $0.552(0.56)$ \\
\hline $20(7-7: 45 p m)$ & $28.02(0.13) \oplus$ & $-0.74(0.48)$ & $0.39(0.49)$ & $1.15(0.505)$ ** & $0.49(0.49)$ \\
\hline 21 (8-8:45pm) & $26.51(0.11) \oplus$ & $-0.9(0.41)^{*}$ & $0.21(0.42)$ & $-0.194(0.425)$ & $0.429(0.42)$ \\
\hline 22 (9-9:45pm) & $25.32(0.10) \oplus$ & $-0.88(0.36)^{*}$ & $0.06(0.37)$ & $-1.165(0.371) \oplus$ & $0.376(0.37)$ \\
\hline 23 (10-10:45pm) & $24.39(0.09) \oplus$ & $-0.79(0.33)^{*}$ & $-0.06(0.34)$ & $-1.796(0.341) \oplus$ & $0.331(0.34)$ \\
\hline $24(11-11: 45 p m)$ & $23.67(0.08) \oplus$ & $-0.69(0.31)^{\star}$ & $-0.14(0.32)$ & $-2.186(0.321) \oplus$ & $0.288(0.32)$ \\
\hline
\end{tabular}

Since the hourly soil temperature at $5 \mathrm{~cm}$ bgs showed statistical significance for the estimates of the probability, would the soil 
temperatures at $25 \mathrm{~cm}$ bgs differentiate the soil temperature response in the ground due to having the buried objects? The temperature sensors at $25 \mathrm{~cm}$ below the ground are positioned just above the DM and DP and just below the SM and SP objects. Similar multilevel model processes were performed with SoilTemp25 as an outcome using the nested observations at level 2 with the Probe-id variable for individual hourly contrasts and Object as level-1 predictor. The individual hourly contrast model for time $(1,2, \ldots 24)$ of day provides an estimate of the probability of the intercept for soil temperatures at $25 \mathrm{~cm}$ and slope of the Object from o (no object) to 1 (with object) for the corresponding contrasts (Table 10). For all contrasts, the estimated intercepts for SoilTemp25 have statistical significance $(p<0.01)$ and are the same within the specific time of day. For example, the intercept from 0700 to 0745 is $24.53^{\circ} \mathrm{C}$, which is the mean soil temperature probability distribution that controls the effects for the particular time of day.

The slope estimates have statistical significance at certain times of the day for all contrasts (Table 10). A negative slope means the temperature is cooling during that time of the day, while a positive slope means that there is warming relative to the intercept due to the influence of having the objects in the ground. For example, the soil temperature below the SP at time code 8 from 0700 to 0745 is approximately $0.9^{\circ} \mathrm{C}$ relative to the intercept value of $24.53^{\circ} \mathrm{C}$ (about $25.4^{\circ} \mathrm{C}$ below the SP object) and about $-0.4^{\circ} \mathrm{C}$ relative to the intercept value of $24.53^{\circ} \mathrm{C}$ for the DP object (about $24.1^{\circ} \mathrm{C}$ above the DP object). Also, at 0700 to 0745 , the ground is warmer below the SP object than the SM object. Overall, the soil temperature below the SM object is relatively cooler than undisturbed areas. The DP object is differentiated by soil temperature at $25 \mathrm{~cm}$ from 0900 to 1145 with negative slopes ranging from -0.52 to $-0.57^{\circ} \mathrm{C}$ and from 1800 to 0945 with positive change between 0.81 and $0.92^{\circ} \mathrm{C}$. There is a very narrow window to differentiate the DM object between 1500 and 1845 with a slope value of $0.5^{\circ} \mathrm{C}$ relative to the intercept values of soil temperature at $25 \mathrm{~cm}$ for those times. 
Table 10. Model $\mathrm{C}$ results of probability estimates and standard errors (values in parenthesis) from multilevel model of applying the PROC MIXED procedures aggregated based on time of the day contrasts with intercept of soil temperature at $25 \mathrm{~cm}$ (SoilTemp) as a dependent variable.

\begin{tabular}{|c|c|c|c|c|c|}
\hline \multirow[t]{3}{*}{ Time of day } & \multirow{3}{*}{$\begin{array}{c}\text { Intercept } \\
\text { (SoilTemp25) }\end{array}$} & \multicolumn{4}{|c|}{ Contrast (Objects versus without) } \\
\hline & & SM & DM & SP & DP \\
\hline & & \multicolumn{4}{|c|}{ Slope of Object } \\
\hline 1 (12-12:45am) & $26.2(0.07)^{\oplus}$ & $-0.87(0.26)^{\oplus}$ & $0.121(0.259)$ & $-0.482(0.253) \dagger$ & $0.49(0.264)^{\dagger}$ \\
\hline $2(1-1: 45 a m)$ & $25.95(0.07)^{\oplus}$ & $-0.85(0.25)^{\oplus}$ & $0.092(0.251)$ & $-0.205(0.246)$ & $0.34(0.255)$ \\
\hline $3(2-2: 45 a m)$ & $25.7(0.07)^{\oplus}$ & $-0.84(0.24)^{\oplus}$ & $0.066(0.244)$ & $0.049(0.239)$ & $0.195(0.247)$ \\
\hline 4 (3-3:45am) & $25.44(0.06)^{\oplus}$ & $-0.82(0.23)^{\oplus}$ & $0.048(0.237)$ & $0.268(0.233)$ & $0.05(0.241)$ \\
\hline 5 (4-4:45am) & $25.2(0.06)^{\oplus}$ & $-0.80(0.23)^{\oplus}$ & $0.041(0.231)$ & $0.463(0.228)^{*}$ & $-0.07(0.234)$ \\
\hline $6(5-5: 45 a m)$ & $24.96(0.06)^{\oplus}$ & $-0.78(0.22)^{\oplus}$ & $0.033(0.227)$ & $0.627(0.223)^{\oplus}$ & $-0.19(0.229)$ \\
\hline 7 (6-6:45am) & $24.74(0.06)^{\oplus}$ & $-0.75(0.22)^{\oplus}$ & $0.035(0.223)$ & $0.764(0.22)^{\oplus}$ & $-0.30(0.225)$ \\
\hline $8(7-7: 45 a m)$ & $24.53(0.06)^{\oplus}$ & $-0.71(0.22)^{\oplus}$ & $0.037(0.22)$ & $0.877(0.217)^{\oplus}$ & $-0.41(0.223)$ \\
\hline 9 (8-8:45am) & $24.34(0.06)^{\oplus}$ & $-0.61(0.22)^{\oplus}$ & $0.047(0.219)$ & $0.955(0.216)^{\oplus}$ & $-0.49(0.221)^{\star}$ \\
\hline 10 (9-9:45am) & $24.22(0.06)^{\oplus}$ & $-0.46(0.22)^{*}$ & $0.074(0.218)$ & $0.969(0.215)^{\oplus}$ & $-0.55(0.22)^{*}$ \\
\hline 11 (10-10:45am) & $24.19(0.06)^{\oplus}$ & $-0.27(0.22)$ & $0.115(0.218)$ & $0.885(0.215)^{\oplus}$ & $-0.57(0.22)^{\star}$ \\
\hline 12 (11-11:45am) & $24.27(0.06)^{\oplus}$ & $-0.07(0.21)$ & $0.184(0.216)$ & $0.695(0.213)^{\oplus}$ & $-0.52(0.218)^{*}$ \\
\hline $13(12-12: 45 p m)$ & $24.48(0.06)^{\oplus}$ & $0.09(0.21)$ & $0.267(0.214)$ & $0.395(0.211)^{*}$ & $-0.42(0.215)^{\dagger}$ \\
\hline $14(1-1: 45 p m)$ & $24.80(0.06)^{\oplus}$ & $0.16(0.22)$ & $0.355(0.217)$ & $0.011(0.217)$ & $-0.24(0.217)$ \\
\hline $15(2-2: 45 p m)$ & $25.19(0.06)^{\oplus}$ & $0.13(0.23)$ & $0.436(0.226)^{\dagger}$ & $-0.417(0.226)^{\dagger}$ & $-0.02(0.226)$ \\
\hline $16(3-3: 45 p m)$ & $25.61(0.06)^{\oplus}$ & $0.03(0.24)$ & $0.49(0.235)^{*}$ & $-0.834(0.235)^{\oplus}$ & $0.23(0.235)$ \\
\hline $17(4-4: 45 p m)$ & $26.01(0.06)^{\oplus}$ & $-0.11(0.24)$ & $0.53(0.24)^{*}$ & $-1.186(0.24)^{\oplus}$ & $0.46(0.24)^{\dagger}$ \\
\hline $18(5-5: 45 p m)$ & $26.35(0.07)^{\oplus}$ & $-0.27(0.24)$ & $0.53(0.247)^{*}$ & $-1.445(0.243)^{\oplus}$ & $0.66(0.25)^{*}$ \\
\hline $19(6-6: 45 p m)$ & $26.61(0.07)^{\oplus}$ & $-0.42(0.26)$ & $0.50(0.259)^{\dagger}$ & $-1.596(0.252)^{\oplus}$ & $0.81(0.26)^{*}$ \\
\hline $20(7-7: 45 p m)$ & $26.78(0.07)^{\oplus}$ & $-0.58(0.27)^{*}$ & $0.453(0.27)^{\dagger}$ & $-1.63(0.262)^{\oplus}$ & $0.89(0.28)^{*}$ \\
\hline 21 (8-8:45pm) & $26.83(0.07)^{\oplus}$ & $-0.72(0.27)^{\oplus}$ & $0.387(0.276)$ & $-1.545(0.268)^{\oplus}$ & $0.92(0.28)^{*}$ \\
\hline 22 (9-9:45pm) & $26.77(0.07)^{\oplus}$ & $-0.81(0.27)^{\oplus}$ & $0.308(0.277)$ & $-1.35(0.27)^{\oplus}$ & $0.875(0.28)$ * \\
\hline 23 (10-10:45pm) & $26.62(0.07)^{\oplus}$ & $-0.86(0.27)^{\oplus}$ & $0.229(0.274)$ & $-1.084(0.267)^{\oplus}$ & $0.77(0.28)^{*}$ \\
\hline $24(11-11: 45 p m)$ & $26.41(0.07)^{\oplus}$ & $-0.87(0.26)^{\oplus}$ & $0.163(0.268)$ & $-0.786(0.262)^{\oplus}$ & $0.64(0.27)^{*}$ \\
\hline
\end{tabular}

\footnotetext{
${ }^{\oplus} p<.01 ;{ }^{\star} p<.05 ; \dagger p<.10$.
} 


\subsubsection{Model C: Surface temperature with IR as outcome}

Based on the results from the logistic regression model (Table 6), the estimated probabilities indicated a statistical significance for the Object with surface temperature (IR_tir). Using similar multilevel model processes from previous analyses for soil temperature, the surface temperature derived from IR measurements (IR_tir) is used as an outcome with nested observations at level 2 with Probe-id variable and Object as level-1 predictor. The individual hourly contrast model for time of day $(1,2, \ldots 24)$ estimates the probability of the intercepts for IR temperatures (IR_tir) and slopes of the Object for the corresponding contrasts (Table 11). The results indicate the hourly average IR for surface temperatures $\left(I R \_\right.$tir $)$are statistically significant $(p<.01)$ with estimated intercepts ranging between 19.8 and $35.9^{\circ} \mathrm{C}$. The warmest mean surface temperature probability distribution based on the estimated intercept is between 1300 and 1345 at $35.87^{\circ} \mathrm{C}$.

The surface temperature change above the SP object around 2400 is approximately $-1.6^{\circ} \mathrm{C}$ relative to the intercept value of $20.98^{\circ} \mathrm{C}$ (which is approximately $19.4^{\circ} \mathrm{C}$ for the SP). The contrast for the SP object around 2400 is approximately $1^{\circ} \mathrm{C}$ cooler than for the SM object. At midday (1200 to 1245 ), the soil surface above the SP object is warmer by $1.7^{\circ} \mathrm{C}$ than the contrast for the SM object.

The estimated slope values for the SM object for most of the 24-hour cycle have statistical significance for detecting the contrast. The SM object contrasts are detectible with the lowest cooling unit change of -0.62 to $-0.76^{\circ} \mathrm{C}$ from 2000 to 1245 and highest ranges of warming unit change of 1 to $1.3^{\circ} \mathrm{C}$ from 1100 to $14: 45$. The DM object contrast appears measurable by thermal IR temperature based on the surface warming unit change of 2.2 to $3.0^{\circ} \mathrm{C}$ from 1100 to 1545 . There is a very a narrow window to detect the DM object contrast between 1200 and 1545 with slope values of $0.6^{\circ} \mathrm{C}$ relative to the intercept values of surface temperature at those times. Overall, the temperature change for SP object contrast is more noticeable or detectable most of the 24-hr cycle than for other objects, with the largest range of warming between 2.2 and $3^{\circ} \mathrm{C}$ from 1100 to 1645 . 
Table 11. Model $D$ results of probability estimates and standard errors (values in parenthesis) from multilevel model of applying the PROC MIXED procedures aggregated based on time of the day contrasts with intercept of soil temperature radiance (IR_tir) as a dependent variable.

\begin{tabular}{|c|c|c|c|c|c|}
\hline \multirow[t]{3}{*}{ Time of day } & \multirow{3}{*}{$\begin{array}{l}\text { Intercept } \\
\text { (IR_tir) }\end{array}$} & \multicolumn{4}{|c|}{ Contrast (Objects versus without) } \\
\hline & & SM & DM & SP & DP \\
\hline & & \multicolumn{4}{|c|}{ Slope of Object } \\
\hline 1 (12-12:45am) & $20.98(0.02)^{\oplus}$ & $-0.62(0.06)^{\oplus}$ & $-0.36(0.06)^{\oplus}$ & $-1.60(0.06)^{\oplus}$ & $-0.24(0.06)^{\oplus}$ \\
\hline $2(1-1: 45 a m)$ & $20.56(0.02)^{\oplus}$ & $-0.56(0.06)^{\oplus}$ & $-0.38(0.06)^{\oplus}$ & $-1.66(0.06)^{\oplus}$ & $-0.25(0.06)^{\oplus}$ \\
\hline $3(2-2: 45 a m)$ & $20.33(0.02)^{\oplus}$ & $-0.5(0.06)^{\oplus}$ & $-0.38(0.06)^{\oplus}$ & $-1.65(0.06)^{\oplus}$ & $-0.25(0.06)^{\oplus}$ \\
\hline 4 (3-3:45am) & $20.14(0.02)^{\oplus}$ & $-0.45(0.06)^{\oplus}$ & $-0.38(0.06)^{\oplus}$ & $-1.63(0.06)^{\oplus}$ & $-0.26(0.06)^{\oplus}$ \\
\hline 5 (4-4:45am) & $19.96(0.02)^{\oplus}$ & $-0.4(0.06)^{\oplus}$ & $-0.37(0.06)^{\oplus}$ & $-1.58(0.06)^{\oplus}$ & $-0.26(0.06)^{\oplus}$ \\
\hline $6(5-5: 45 a m)$ & $19.82(0.02)^{\oplus}$ & $-0.36(0.06)^{\oplus}$ & $-0.36(0.06)^{\oplus}$ & $-1.53(0.06)^{\oplus}$ & $-0.27(0.06)^{\oplus}$ \\
\hline 7 (6-6:45am) & $20.15(0.02)^{\oplus}$ & $-0.27(0.06)^{\oplus}$ & $-0.3(0.06)^{*}$ & $-1.4(0.06)^{\oplus}$ & $-0.23(0.06)$ \\
\hline $8(7-7: 45 a m)$ & $21.66(0.04)^{\oplus}$ & $-0.06(0.09)$ & $-0.12(0.09)$ & $-1.03(0.09)^{\oplus}$ & $-0.11(0.09)$ \\
\hline 9 (8-8:45am) & $24.15(0.05)^{\oplus}$ & $0.22(0.12)$ & $0.08(0.12)$ & $-0.39(0.12)^{\oplus}$ & $0.04(0.12)$ \\
\hline $10(9-9: 45 a m)$ & $26.95(0.07)^{\oplus}$ & $0.56(0.18)^{\oplus}$ & $0.24(0.18)$ & $0.41(0.18)$ & $0.17(0.18)$ \\
\hline 11 (10-10:45am) & $30.18(0.1)^{\oplus}$ & $0.93(0.24)^{\oplus}$ & $0.45(0.24)$ & $1.28(0.24)^{\oplus}$ & $0.39(0.24)$ \\
\hline 12 (11-11:45am) & $33.02(0.11)^{\oplus}$ & $1.2(0.28)^{\oplus}$ & $0.67(0.28)^{\star}$ & $2.16(0.28)^{\oplus}$ & $0.59(0.28)^{*}$ \\
\hline $13(12-12: 45 p m)$ & $35.04(0.12)^{\oplus}$ & $1.27(0.3)^{\oplus}$ & $0.75(0.3)^{*}$ & $2.76(0.3)^{\oplus}$ & $0.63(0.3)^{*}$ \\
\hline 14 (1-1:45pm) & $35.87(0.12)^{\oplus}$ & $1.2(0.29)^{\oplus}$ & $0.69(0.29)^{\star}$ & $3(0.29)^{\oplus}$ & $0.65(0.29)^{\oplus}$ \\
\hline $15(2-2: 45 p m)$ & $35.75(0.11)^{\oplus}$ & $1(0.27)^{\oplus}$ & $0.68(0.27)^{*}$ & $2.99(0.27)^{\oplus}$ & $0.64(0.27)^{*}$ \\
\hline $16(3-3: 45 p m)$ & $34.96(0.09)^{\oplus}$ & $0.69(0.22)^{\oplus}$ & $0.71(0.22)^{\oplus}$ & $2.76(0.22)^{\oplus}$ & $0.62(0.22)^{\star}$ \\
\hline 17 (4-4:45pm) & $33.73(0.07)^{\oplus}$ & $0.44(0.17)^{\star}$ & $0.63(0.17)^{\oplus}$ & $2.36(0.17)^{\oplus}$ & $0.58(0.17)^{*}$ \\
\hline $18(5-5: 45 p m)$ & $31.86(0.05)^{\oplus}$ & $0.13(0.12)$ & $0.44(0.12)^{*}$ & $1.83(0.12)^{\oplus}$ & $0.46(0.12)^{*}$ \\
\hline $19(6-6: 45 p m)$ & $29.59(0.03)^{\oplus}$ & $-0.25(0.08)$ & $0.22(0.08)^{*}$ & $1.1(0.08)^{\oplus}$ & $0.22(0.08)$ \\
\hline 20 (7-7:45pm) & $26.57(0.03)^{\oplus}$ & $-0.55(0.07)^{\oplus}$ & $-0.04(0.07)$ & $0.34(0.07)^{\oplus}$ & $-0.02(0.07)$ \\
\hline 21 (8-8:45pm) & $24.39(0.03)^{\oplus}$ & $-0.73(0.07)^{\oplus}$ & $-0.18(0.07)$ & $-0.4(0.07)^{\oplus}$ & $-0.13(0.07)$ \\
\hline 22 (9-9:45pm) & $22.95(0.03)^{\oplus}$ & $-0.76(0.07)^{\oplus}$ & $-0.26(0.07)^{\oplus}$ & $-0.94(0.07)^{\oplus}$ & $-0.19(0.07)$ \\
\hline $23(10-10: 45 p m)$ & $22.11(0.03)^{\oplus}$ & $-0.73(0.06)^{\oplus}$ & $-0.31(0.06)^{\oplus}$ & $-1.27(0.06)^{\oplus}$ & $-0.22(0.06)^{\oplus}$ \\
\hline 24 (11-11:45pm) & $21.50(0.03)^{\oplus}$ & $-0.67(0.06)^{\oplus}$ & $-0.34(0.06)^{\oplus}$ & $-1.48(0.06)^{\oplus}$ & $-0.23(0.06)^{\oplus}$ \\
\hline
\end{tabular}

${ }^{\oplus} p<.01 ;{ }^{\star} p<.05 ; \dagger p<.10$.

Random estimates for SM contrast within-Probe-id variance of $10^{\circ} \mathrm{C}$ at midnight and $38^{\circ} \mathrm{C}$ at $2 \mathrm{pm}$, between-Probe-id variance of $0^{\circ} \mathrm{C}$ at midnight and $0.07^{\circ} \mathrm{C}$ noontime.

Random estimates for SP contrast within-Probe-id variance of $10^{\circ} \mathrm{C}$ at midnight and $41^{\circ} \mathrm{C}$ at $2 \mathrm{pm}$, between-Probe-ids variance of $0^{\circ} \mathrm{C}$ at midnight and $0.06^{\circ} \mathrm{C}$ noontime. 
Figure 9 shows the comparison of responses between the IR temperature values and soil temperature at $5 \mathrm{~cm}$. Each contrast corresponds with the estimated random intercept and the slope or unit change of IR temperature (left) and soil temperature (right). For all contrasts, the estimated intercepts (dashed line) for thermal IR temperature values are higher during the daytime by approximately $4^{\circ} \mathrm{C}$ and $2^{\circ} \mathrm{C}$ cooler at night than at $5 \mathrm{~cm}$ in the soil. However, the divergence due to the unit incremental change or the slope (solid line) varies for detecting the contrasts. The most pronounced divergence for detecting the contrast with background is with the buried SM object (Figure $9 a$ ) relative to no object, with the highest change of $5.5^{\circ} \mathrm{C}$ and $3^{\circ} \mathrm{C}$ soil temperature at $5 \mathrm{~cm}$ and thermal IR temperatures, respectively.

The SM and SP object contrasts (Figure 9c) show statistical significance (p $<0.01$ ) when the intercept (dashed line) and difference due to the temperature change or the slope estimates (solid line) for the buried object relative to no object are significantly diverging, while the divergences for the DM and DP objects are not as notable (Figures $9 b$ and $9 d$, respectively).

Figure 9. Probability estimates of intercept and slope of the hourly IR surface and soil temperatures at $5 \mathrm{~cm}$ for the four contrasts (a) SM, (b) DM, (c) SP, and (d) DP.

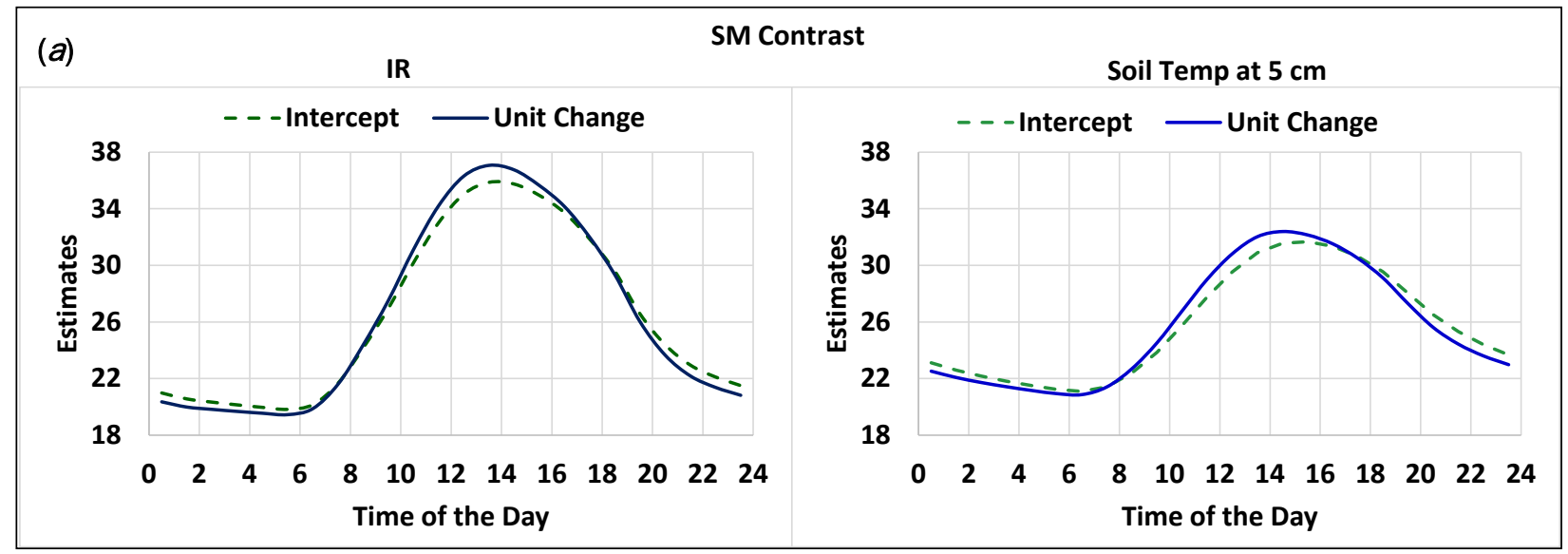



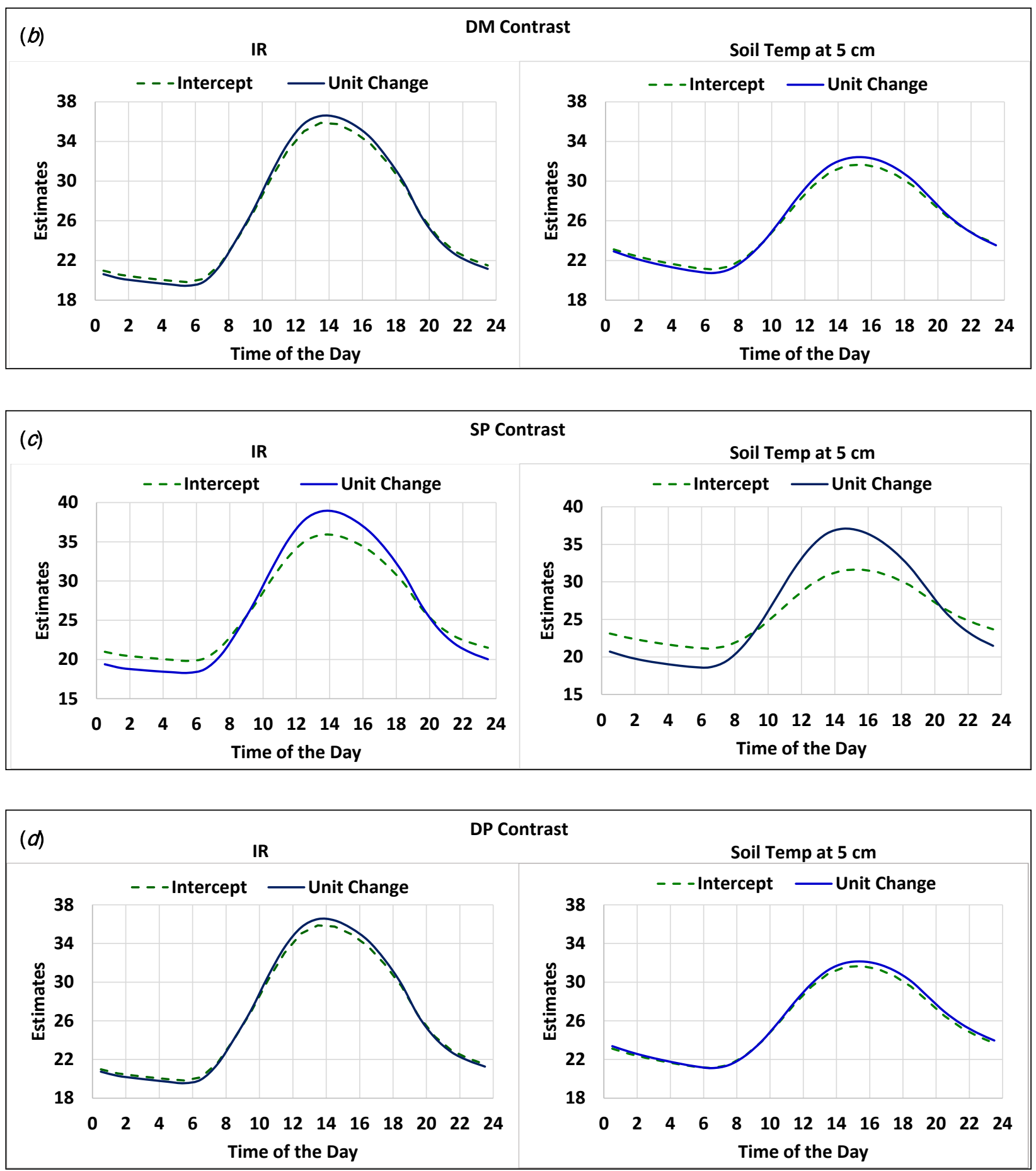

\subsubsection{Model D: Soil moisture as outcome}

The probability estimates of the intercepts (Figure 10a-d) for the hourly contrasts of soil moisture (Soil15) are statistically significant $(p<0.01)$. The slope estimates of the Object for the corresponding SM and SP contrasts have no statistical significance, meaning $p>0.50$. On average 
with having the SM object, the soil was $2 \%$ by volume wetter than surrounding soil without any object (Figure 10). On the other hand, the soil on the side of the SP object was approximately $1 \%$ by volume drier than surrounding soil without an object. This contradictory outcome is likely due to the moisture sensor positioned on the side of the objects. However, the slope estimates of the Object for the corresponding DM and DP object contrasts have statistical significance $(p<0.01)$ with values lower than the estimated intercepts. These indicate that overall soil moisture throughout the day is approximately $4-5 \%$ by volume lower on top of the DM and DP objects than surrounding soil with no objects. In this case, having the soil moisture sensors positioned $10 \mathrm{~cm}$ above objects provided a consistent response.

Figure 10. Estimates of intercept and slope comparison for the hourly IR surface and soil temperature for the four contrasts (a) SM, (b) SP, (c) DM, and (d) SP.
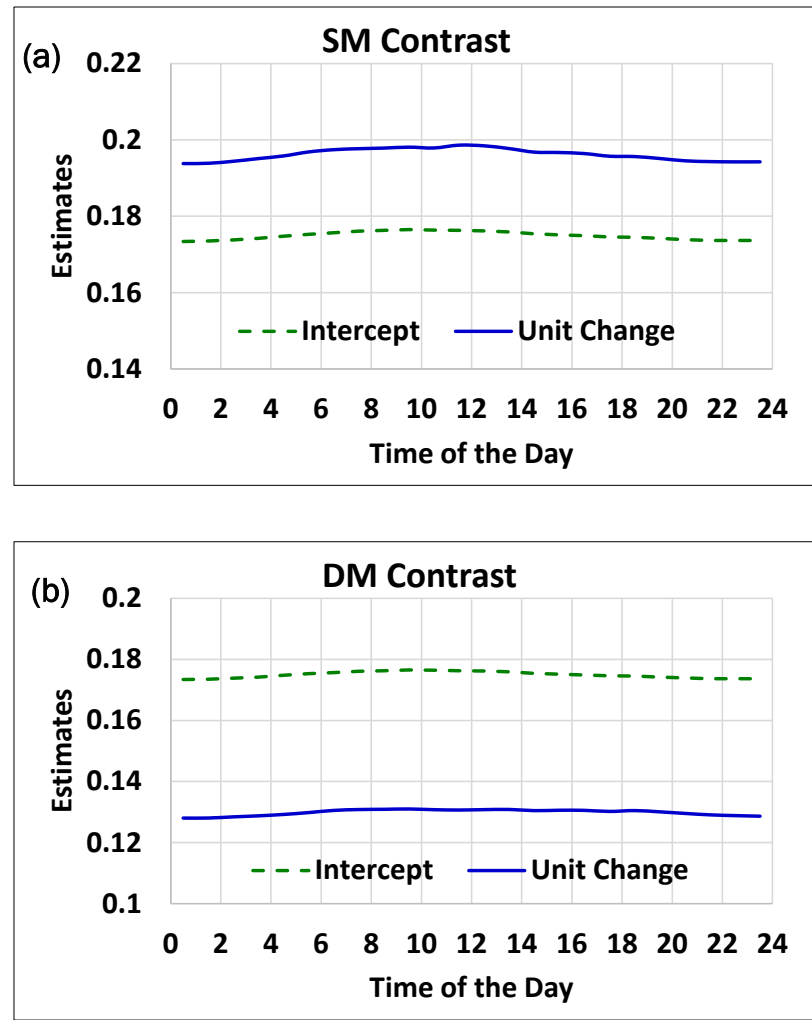

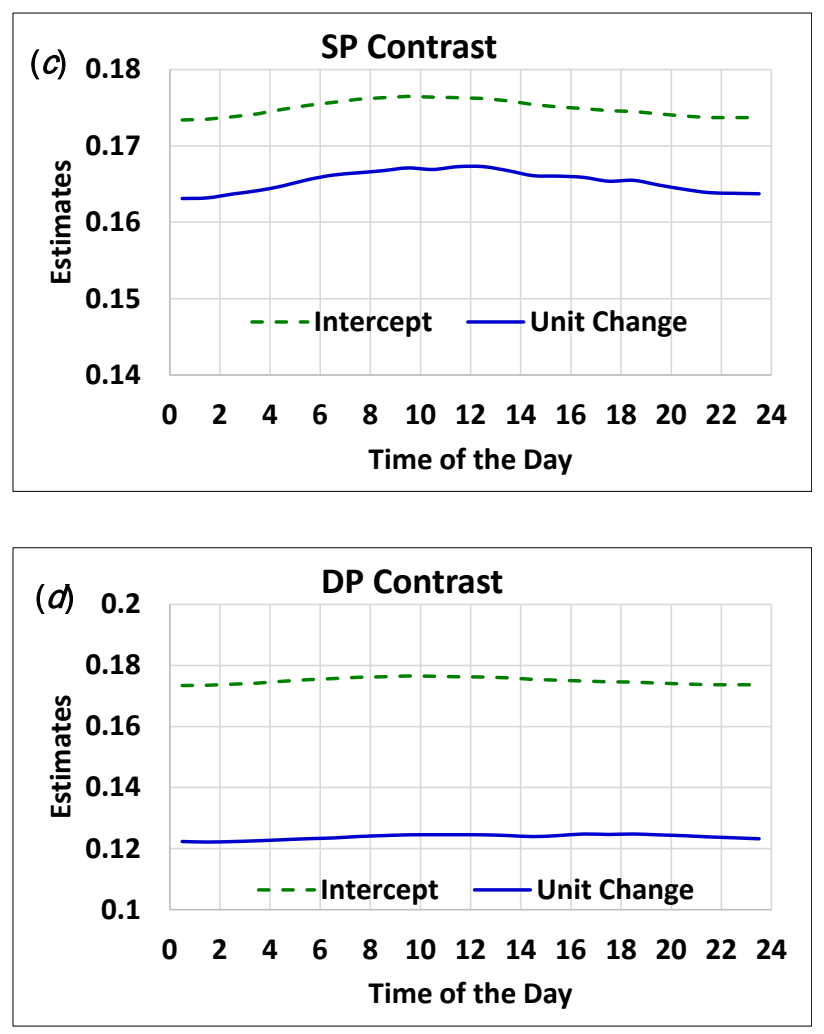

\subsection{ROC analysis}

Receiver operating characteristic (ROC) curves are useful for assessing the PD and FAR associated with object detection (Zoe et al. 2007; SAS 9.4). Incorporating ROC curves into our analysis of objective visibility using multilevel modeling allows for a single measure of comparison. One of the simplest scenarios for prediction is the case of a binary predictor. The most-used such measure is the area under the ROC curve (AUC). In general, an AUC of 0.5 suggests no distinction (i.e., ability to differentiate with and without the objects), $0.6-0.7$ is considered marginal, $0.7-0.8$ is considered acceptable, $0.8-0.9$ is considered excellent, and more than 0.9 is considered outstanding (Hosmer and Lemeshow 2000; Mandrekar 2010). ROC curve comparisons in terms of AUC are conducted using the PROC Logistics procedure (SAS 2020). The AUC analysis of the contrasts included the hourly surface temperature (IR_tir labeled as IR temp), soil temperature at $5 \mathrm{~cm}$ (SoilTemp5 labeled in the plot as Soil temp), and soil moisture (Soil15 renamed in the plot as Soil MC) for Figure 4.5. An AUC composite of these three variables for the hourly SP object contrasts is also included in the analytical model (labeled in the Figure 7 plot as Model). Examples of the ROC plots for the corresponding object contrasts are shown in Figure 11, showing approximately the same AUC values at 1300 
to 1345 and 1200 to 1245 . The soil moisture-impacted AUC values are relatively uniform (Figure 12) throughout the 24-hour cycle, which matches undeviating patterns of the intercepts and slope estimates (Figure 10). This is due to there being little variation in soil moisture during the study period (15 July-31 August 2018). Otherwise the AUC values of the soil temperature at $5 \mathrm{~cm}$ and IR temperature vary with time (Figures 11 and 12). Particularly for the SM and SP objects, the AUC values for the IR temperature are marginal for differentiating with and without the objects at certain times of day (Figures 10 and 11). In general, the time of day with elevated AUC values for IR and soil temperature are identical with the probability estimates (Figure 9). The composite model provided excellent AUC values when surface temperature, soil temperature at $5 \mathrm{~cm}$, and soil moisture were combined in the prediction for contrast.

Figure 11. ROC curves for the four contrasts (a) SM, (b) DM, (c) SP, and (d) DP at 1300 to 1345 (left) and at 1200 to1245 (right) for the surface temperature (IR_tir labeled as IR temp), soil temperature (SoilTemp5 labeled in the plot as Soil temp), and soil moisture (Soil15 renamed in the plot as Soil MC).

SM Contrast




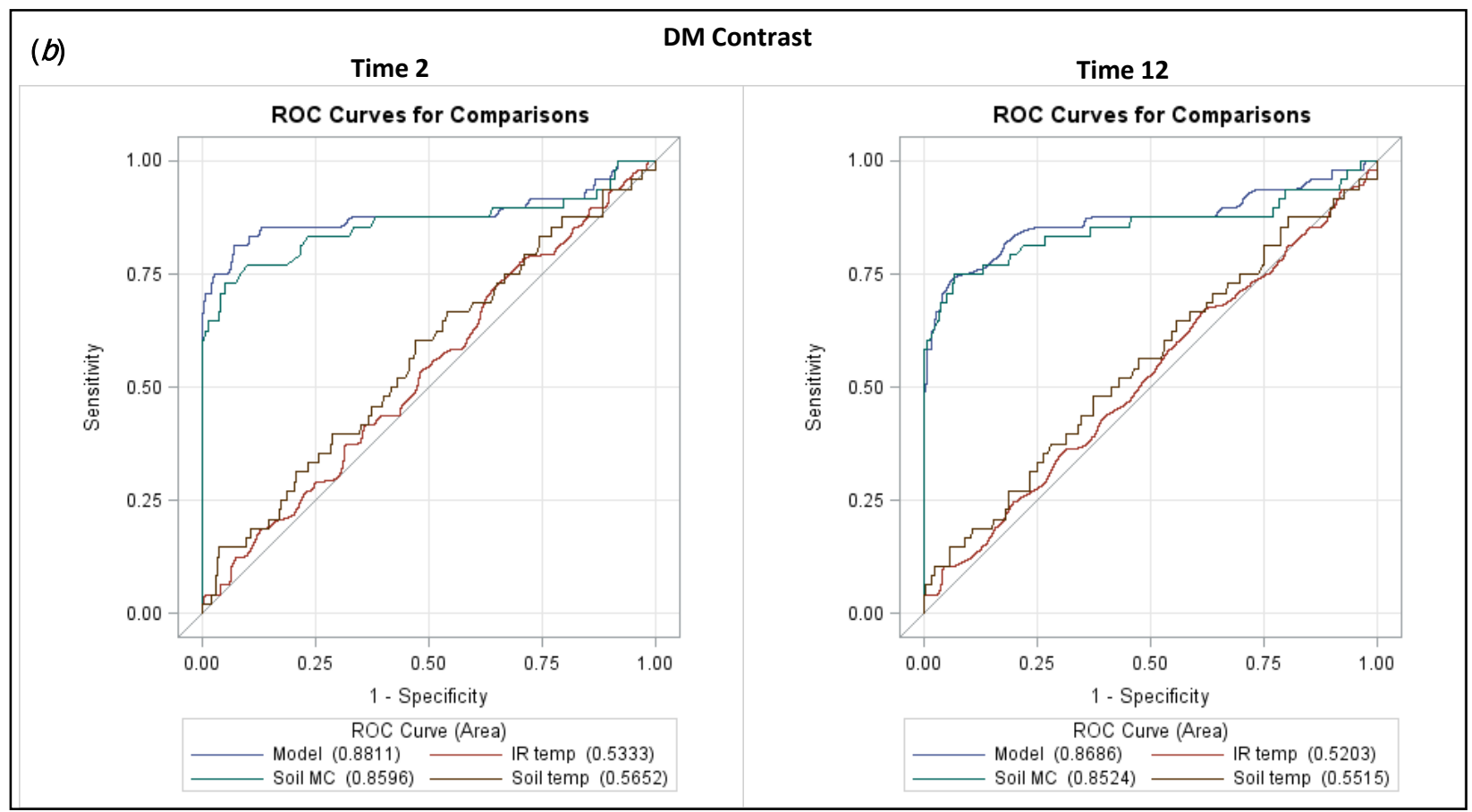

\section{(c)}

Time 2

\section{SP Contrast}

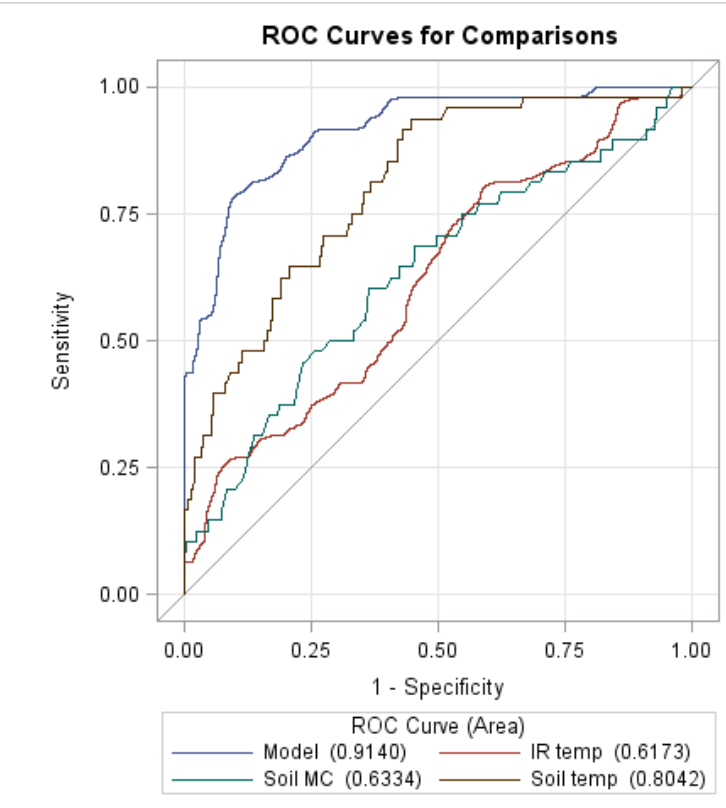

Time 12








Figure 12. Hourly ROC curves comparisons for the (a) SM, (b) DM, (c) SP, and (d) DP contrasts between 15 July and 31 August 2018.

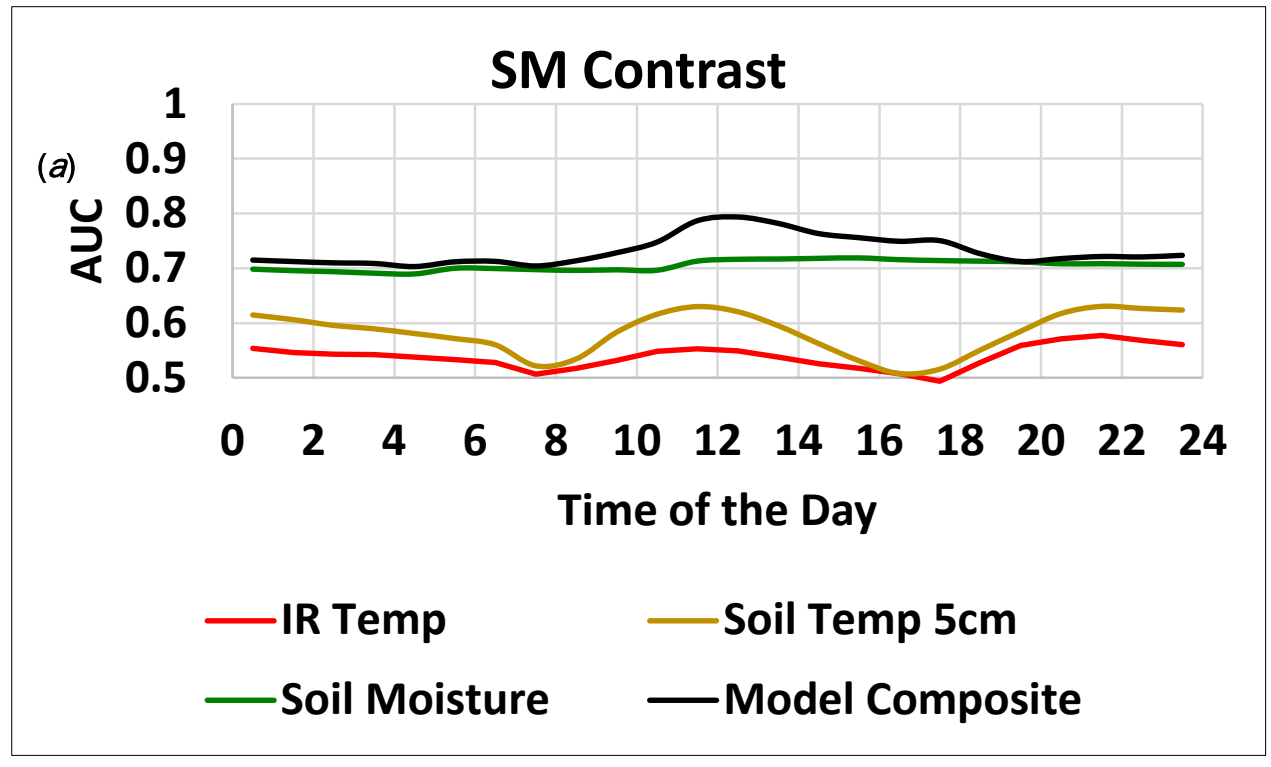



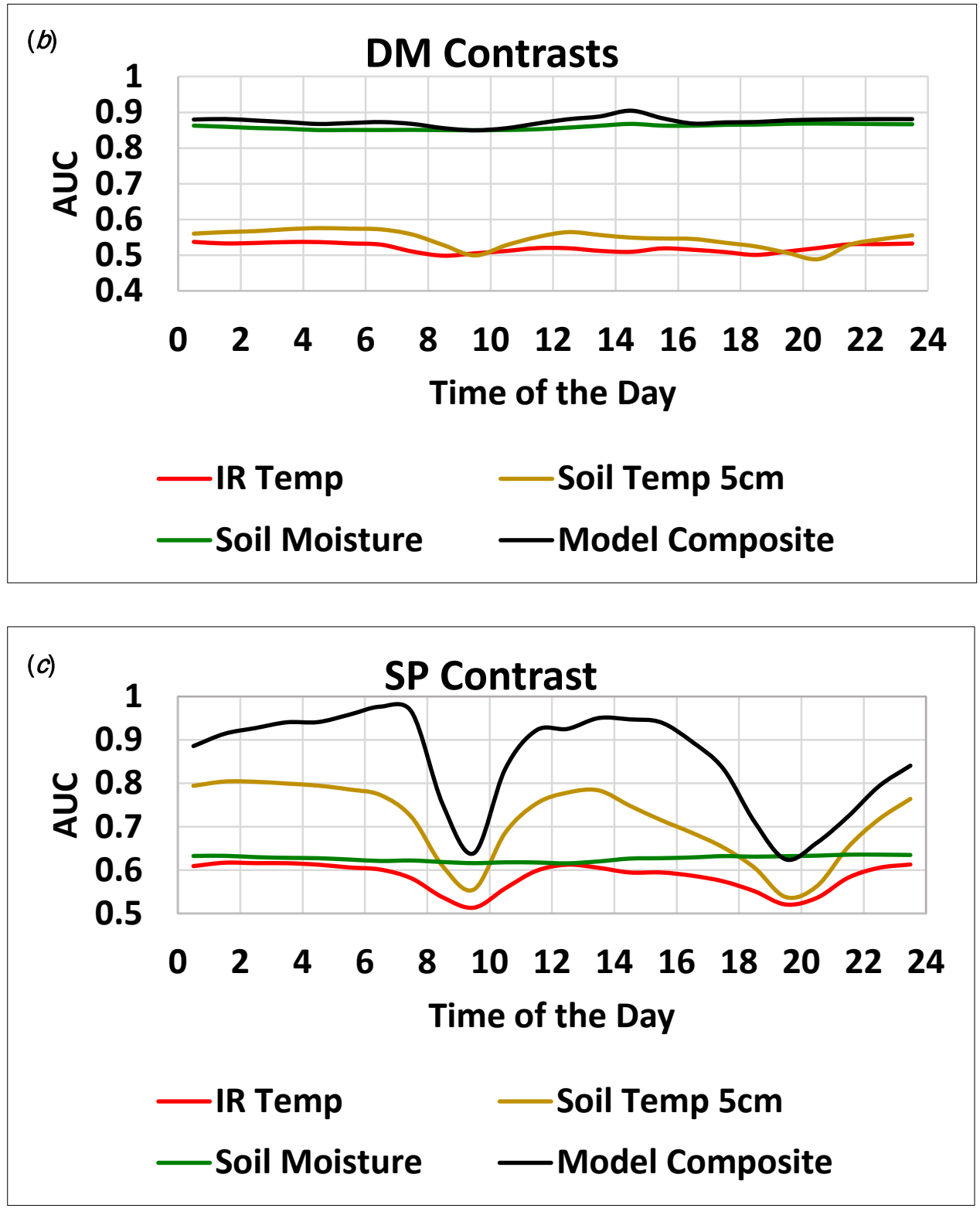


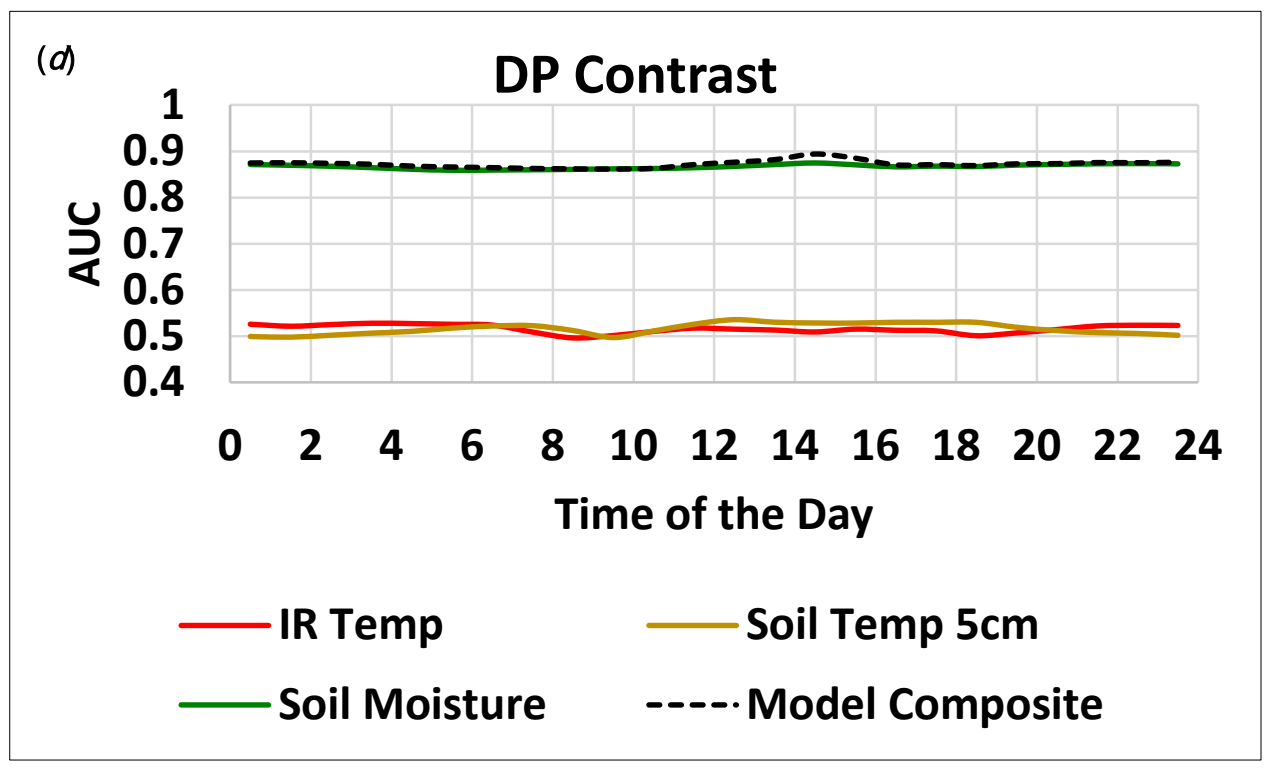

\subsection{Section 4 discussion and summary}

Among the environmental or input measurements, the thermal IR and the soil temperature as well as the soil moisture are the outcome variables that have the probability of differentiating the contrast of the buried objects. Air temperature, relative humidity, SWR, and wind speed are measurements that have no direct impact on buried object contrasts and detectability. Although these measurements are statistically associated with the soil temperatures, the relationship with various slope values is commonly related due to energy balance or the surface/atmosphere radiation and absorption exchange.

Overall, the multi-level modeling approach is able to differentiate and identify the contrast for the objects when taking into environmental phenomenology variables including the thermal IR surface temperature measurements and soil temperature at $5 \mathrm{~cm}$. The object contrast responses differ in temperature magnitude as a function of sinusoidal changes in the 24-hour period. In addition, the divergence between the intercept of the temperature responses and the slope varies for each contrast. The difference in slope for each contrast is due to differences in the rate of temperature change between no buried object and the buried objects. Among all the objects, the contrast for the SP object exhibits the highest probability of detection. The slope estimates have statistical significance primarily for the SP and SM object contrasts, but not particularly for the DM and DP object contrasts. This means that, compared to no object, the SP and SM objects are much more detectable than DP and DM objects 
using the thermal IR temperature. The soil temperature above the SP object is overall warmer during the daytime and cooler at nighttime than the SM object by $4^{\circ} \mathrm{C}$ and $1.7^{\circ} \mathrm{C}$, respectively, relative to soil temperature of areas with no buried objects. The thermal properties of the objects are influencing the temperature contrasts, which are detectable for shallow plastic and shallow metal objects when the ground is warm and cool. When the ground is warming during day light hours, the highest rate of temperature change occurs between 1200 and 1600 yielding the greatest detectability for the SP object. The highest detectability for the SM objects occurs between 1000 and 1400 . Similarly, the surface soil temperature as measured with thermal IR above the SP object is generally warmer (daytime) and cooler (nighttime) than above the metals object by approximately $1.7^{\circ} \mathrm{C}$ and $0.9^{\circ} \mathrm{C}$, respectively, relative to the adjacent background surface soil temperature with no buried objects. The maximum surface soil temperature above the SP objects as measured with thermal IR occurs between 1200 and 1600 for the plastic object and between 1100 and 1400 for the metal object.

In general, the plastic materials have more insulative properties while metal materials are more conductive. In other words, the soil temperatures above the plastic objects heat more quickly in daylight hours and retain the thermal energy instead of diffusing it downward. The temperature effect of the plastic object is very localized and is greatly diminished even $10 \mathrm{~cm}$ away. However, for the deep buried objects the thermal contrasts due to the difference in surface soil temperature as measured with thermal IR are less than $1^{\circ} \mathrm{C}$ during daylight hours and $2.3^{\circ} \mathrm{C}$ during nighttime hours. Thus, the detectability of deeper buried objects, $>5 \mathrm{~cm}$ from the ground surface, with thermal IR is limited.

On average, the soil on the side of the SM object is $2 \%$ by volume wetter than surrounding soil without any object, while the soil on the side of the SP object is approximately $1 \%$ by volume drier than surrounding soil without any object. These results may not be representative moisture conditions on all sides of the objects. The soil moisture above the DM and DP objects contrasts is approximately 4 or $5 \%$ by volume wetter than the surrounding background soil.

ROC curves incorporate observations into a single measure, which defines the responses into a plot of detection probability in the $y$-axis and the corresponding 1 -specificity or FAR in the $x$-axis. Analysis of the AUC for 
the ROC curves provided acceptable ranges of true positive rate versus its false positive rate for soil moisture for differentiating with and without the objects when there were no significant rainfall events and no significant change in soil moisture in the ground. In general, the time of day with the highest thermal FAR for IR and soil temperature is identical with the probability estimates from multilevel model results. 


\section{Thermal Model of Soil Temperature Using Thermal Imagery}

Buried objects have different thermal properties than surrounding soils resulting in localized areas with thermal contrasts above buried objects of different signatures than compared to the surrounding soil. The previous section demonstrated with multilevel modeling the surface soil temperature differences between locations with and without buried objects. The objective of this subtask is to develop a thermal model that can be used to increase the understanding of buried object detectability with thermal IR imagery. Once the model is calibrated and validated it can be used to identify environmental factors affecting thermal response from buried objects. The approach of this initial study involves using data collected during the summer of 2018 at the CRREL test plot (Clausen et al. 2021) to calibrate and validate the model.

\subsection{Methods}

Transient thermal models of the CRREL test plot (Figure 8) were developed using several 2-D soil sections that included no buried object (for control) and metal and plastic objects at two different depths. For this modeling effort, the SVENVIRO module of Soilvision by Bentley Systems Inc., a 1-D/2-D/3-D finite element analysis software, was used. This module is a geotechnical software capable of modeling heat transfer under saturated and unsaturated conditions as well as thermal conduction and convection conditions.

The model was set up as a $65 \mathrm{~cm}$ deep soil section with no flow boundary for the vertical section of the model (Figure 13). The applied boundary conditions utilized measured soil temperatures and thermal imagery temperatures. The soil temperatures measured at $65 \mathrm{~cm}$ bgs were applied to the bottom boundary. The thermal images of the test plot are $320 \times 240$ pixels (Figure 8 ) where each area above each object is $18 \times 17$ pixels. A single pixel of FLIR imagery equates to a $2 \mathrm{~mm}$ area. To average the spatial differences between pixels, the average thermal temperature of 9 pixels centered above each control area and each object was used for the top boundary condition. The thermal imagery temperatures were converted from radiance using the FLIR software where default values were applied during conversion (see Section 4). In addition, several simulations were performed using converted radiances where the thermal imagery 
temperatures were corrected using surface emissivity, air temperature, and relative humidity.

Models were simulated both with no objects (control) and with objects (plastic and metal) at two depths (shallow and deep). The shallow objects were placed with a bottom depth of $25 \mathrm{~cm}$ bgs and the top at $5 \mathrm{~cm}$ bgs. The deeper objects were placed at a bottom depth of $45 \mathrm{~cm}$ bgs and the top at $25 \mathrm{~cm}$ bgs (Figure 13). A calibration dataset was also modeled for five locations (control and objects) using a soil depth from 5 to $60 \mathrm{~cm}$ bgs where the soil temperatures measured at $5 \mathrm{~cm}$ were applied to the top boundary condition (see Figure 13).

Figure 13. Model layout.



BC: Soil T at $65 \mathrm{~cm}$

sensor and model output


BC: Boundary Condition; T: Temperature: TI: Thermal Imagery; ANFO: Ammonium nitrate

A wall thickness of $3 \times 10^{-3} \mathrm{~m}$ and $6 \times 10^{-3} \mathrm{~m}$ was applied to the metal and plastic object, respectively. In the field, the objects were filled with ammonium nitrate (ANFO), which was also added to the objects during the simulations. The soil and object properties are listed in Table 12. 
Table 12. Soil and object properties.

\begin{tabular}{|l|r|r|r|r|}
\hline Material & $\begin{array}{l}\text { Thermal } \\
\text { conductivity } \\
\text { (W/mK) }\end{array}$ & $\begin{array}{l}\text { Volumetric water } \\
\text { content (\%) }\end{array}$ & $\begin{array}{l}\text { Density } \\
\left(\mathrm{kg} / \mathrm{m}^{3}\right)\end{array}$ & $\begin{array}{l}\text { Specific heat } \\
(\mathrm{J} / \mathrm{kgK})\end{array}$ \\
\hline ANFO & 0.11 & 0 & 850 & 1500 \\
\hline 6061 Aluminum & 167 & 0 & 2700 & 897 \\
\hline PVC Type 1 Plastic & 0.19 & 0 & 1379 & 1004 \\
\hline Silty sand & 0.61 to $2.11^{*}$ & 20 & 1876 & 1066 \\
\hline
\end{tabular}

* Temperature dependent and based on laboratory results of samples from field site.

Two time series were simulated using the soil sections as described above: 40 days pre-emplacement (1 June-12 July 2018) and 94 days postemplacement (14 July-15 October 2018). A time-step of 15 minutes was used for the calibration simulations, and a 5-minute time-step was implemented for the thermal imagery temperature simulations.

The model output was validated by comparing the simulated soil temperature to the temperature measurements from thermistors placed spatially in the test plot and at several depths $(5,15,25,35,45,55$, and 65 $\mathrm{cm}$ bgs). Figure 14 shows a top view of the buried object layout and the spatial locations of the temperature sensors at a depth of 5, 25, 45, and 65 $\mathrm{cm}$ bgs. At these depths, thermistor strings were placed in each center of the nine $1 \mathrm{~m} \times 1 \mathrm{~m}$ squares. In the $1 \mathrm{~m} \times 1 \mathrm{~m}$ squares where no objects were placed, controls were assigned to the center $\left(\mathrm{C}_{1}, \mathrm{C}_{2}, \mathrm{C}_{3}, \mathrm{C}_{4}\right.$, and $\left.\mathrm{C}_{5}\right)$. 
Figure 14. Overview of object (shallow plastic $=\mathrm{SP}$; deep plastic $=\mathrm{DP} ;$ shallow metal $=\mathrm{SM}$; deep metal $=\mathrm{DM}$ ) and thermistor placement at a depth of $5,25,45$, and $65 \mathrm{~cm}$.

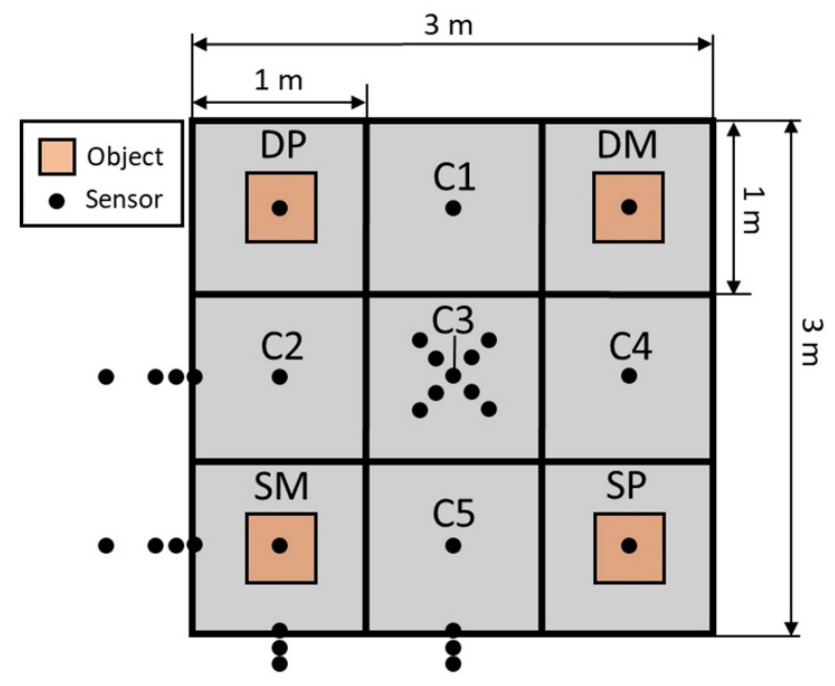

\subsection{Results}

\subsubsection{Temperature variation between pixels}

To investigate the temperature variation between pixels, we investigated the standard deviation of the thermal imagery temperature of nine pixels above the controls and buried objects (Figure 14). The temperature variation ranges from $0.015(\mathrm{SP})$ to $1.9^{\circ} \mathrm{C}(\mathrm{DP})$ for the pre-emplacement period (Figure 15a). The average standard deviation of the thermal imagery temperature ranges from $0.11^{\circ} \mathrm{C}(\mathrm{C} 4)$ to $0.19^{\circ} \mathrm{C}(\mathrm{C} 2)$. The standard deviation varies diurnally where a maximum standard deviation of the thermal imagery temperature is from mid-morning ( 1000) to midafternoon $(\sim 1500)$. On average, the diurnal increase in standard deviation thermal imagery temperature starts early morning $(\sim 0600)$ and ends midevening $(\sim 1900)$.

Similarly, for the post-placement time period there is a diurnal effect on the standard deviation. The standard deviation of the thermal imagery temperature of the nine pixels centered above each control section (C1$\mathrm{C}_{5}$ ) and above each object is about a degree Celsius lower than for the preplacement period and ranges from $0.009^{\circ} \mathrm{C}\left(\mathrm{C}_{3}\right)$ to $0.89^{\circ} \mathrm{C}(\mathrm{C} 1)$. The average standard deviation thermal imagery temperature is also lower than the pre-placement and ranges from $0.082^{\circ} \mathrm{C}(\mathrm{DP})$ to $0.12^{\circ} \mathrm{C}\left(\mathrm{C}_{3}\right)$ (Figure 15b). 
Figure 15. Standard deviation of thermal imagery temperature of nine pixels at the control (C1, C2, C3, C4, and C5) and above each object (shallow plastic, deep plastic, shallow metal, and deep metal) and average for (a) pre-placement of objects (1 June-12 July 2018) and (b) post-placement of objects (14 July-15 October 2018).
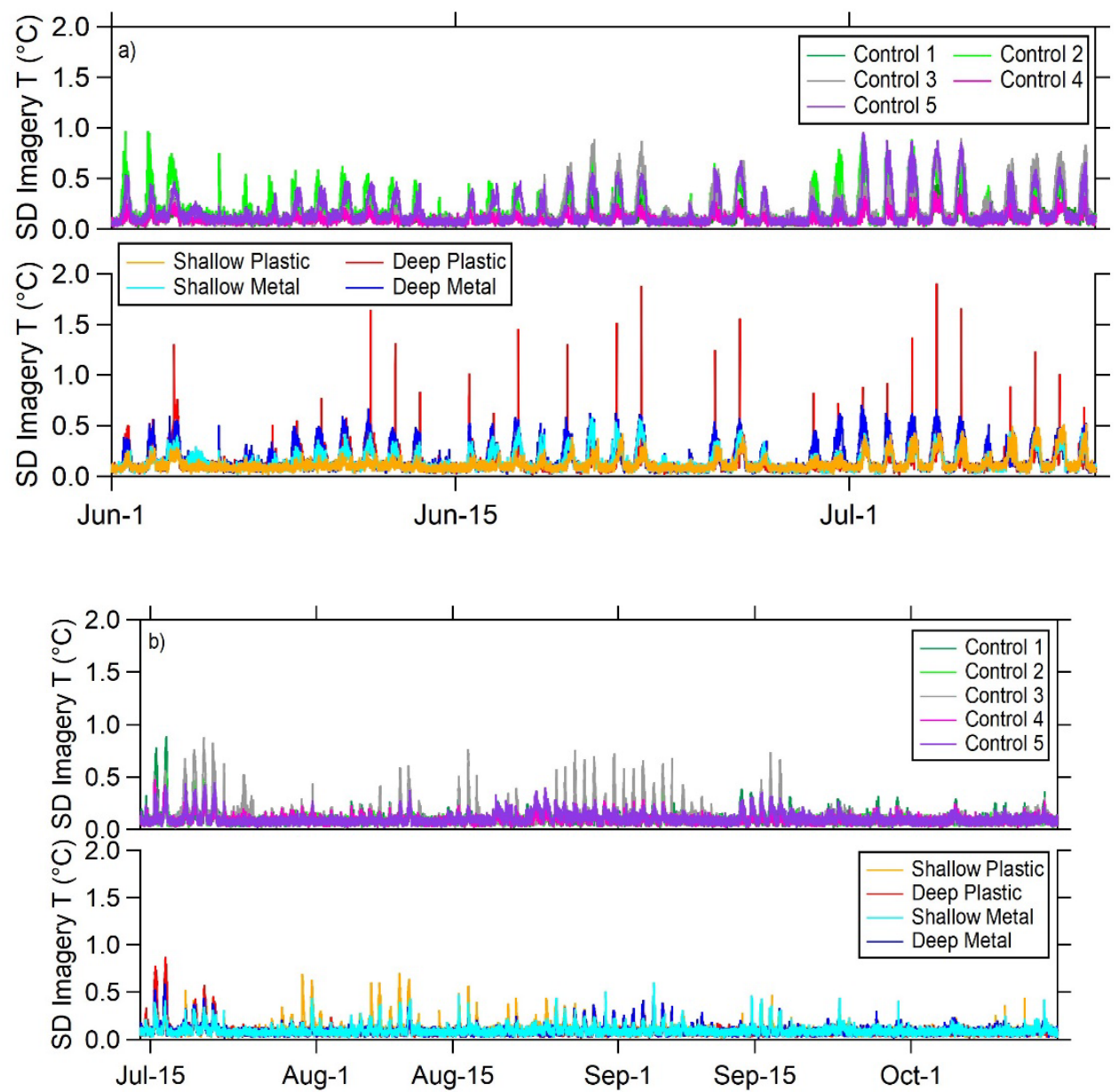

\subsubsection{Pre-emplacement models}

Model calibration simulations were performed for the pre-emplacement time period (40 days), both using the $5 \mathrm{~cm}$ soil temperature and the thermal imagery temperature as the boundary conditions. For the thermal imagery simulations, the average temperatures from nine pixels at the center of each square (Figure 14) was applied to the model. The associated measured soil temperatures at $65 \mathrm{~cm}$ for each square were implemented for the bottom boundary condition. Simulated and measured temperatures from these simulations at the $5 \mathrm{~cm}$ and $25 \mathrm{~cm}$ depths are shown in Figure 16. 
Figure 16. Simulated (BC: thermal imagery temperature and $0.05 \mathrm{~m}$ soil temperature) and measured temperatures at $0.05 \mathrm{~m}$ and $0.25 \mathrm{~m}$ for the (a) control (C3) and four object locations (b) SP, (c) DP, (d) SM, and (e) DM during the object pre-emplacement period (1 June-14 July 2018).

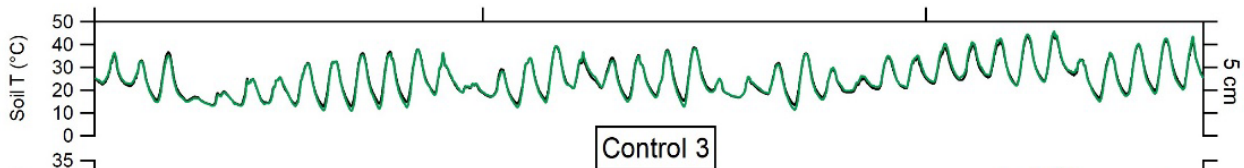

(15)

Shallow Plastic

(Shallow Plastic

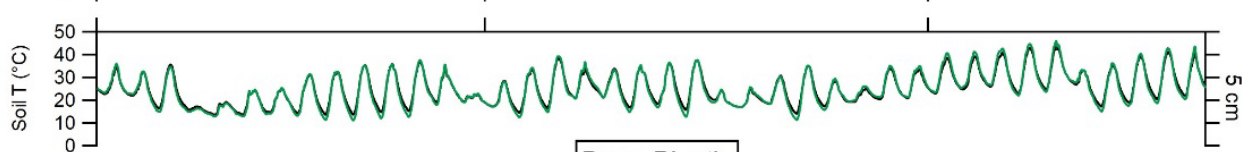

(15)

要

(1)

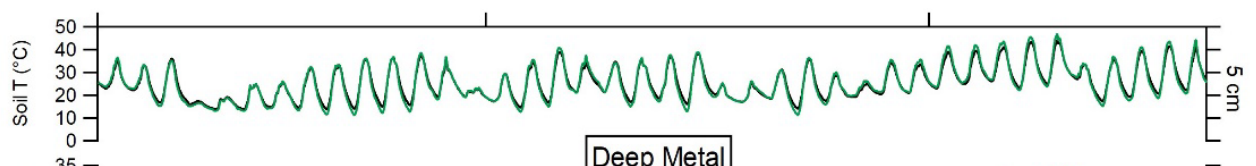

- Sensor - Simulated; BC: Thermal imagery T - Simulated; BC: Soil T 5cm

Regression graphs for these simulations indicate an overall good fit for a total of 3,841 time-steps (Figure 17) with $\mathrm{R}^{2}$ values above 0.98 where 1 represents a perfect fit. The models where the $5 \mathrm{~cm}$ soil temperatures are applied to the surface boundary condition (Figure $17 a, d, g, j$, and $m$ ) have a slightly better goodness of fit at the $25 \mathrm{~cm}$ soil depth than when the thermal imagery temperature is applied to the boundary condition (Figure $17 c, f, i, l$, and $o$ ). Similar results were found at other depths (45 cm and 55 
$\mathrm{cm}$, not shown). The thermal imagery simulations' goodness of fit at $5 \mathrm{~cm}$ bgs (Figure $17 b, e, h, k$, and $n$ ) are slightly lower than at $25 \mathrm{~cm}$ bgs. A negative slope is seen for all $5 \mathrm{~cm}$ simulations where outliers of the simulated soil temperatures from the measured soil temperatures are shown at high and low temperatures. At a soil depth of $5 \mathrm{~cm}$, a higher offset is shown for the DP, SM, and DM compared to shallows, and C3.

Figure 17. Pre-emplacement regression graphs for soil temperature (at 5 and $25 \mathrm{~cm}$ bgs) and simulated for $(a-c)$ Control 3, $(d-f)$ shallow plastic, $(g-l)$ deep plastic, $(j-\hbar)$ shallow metal, and $(m-o)$ deep metal objects $(y=a+b x)$.
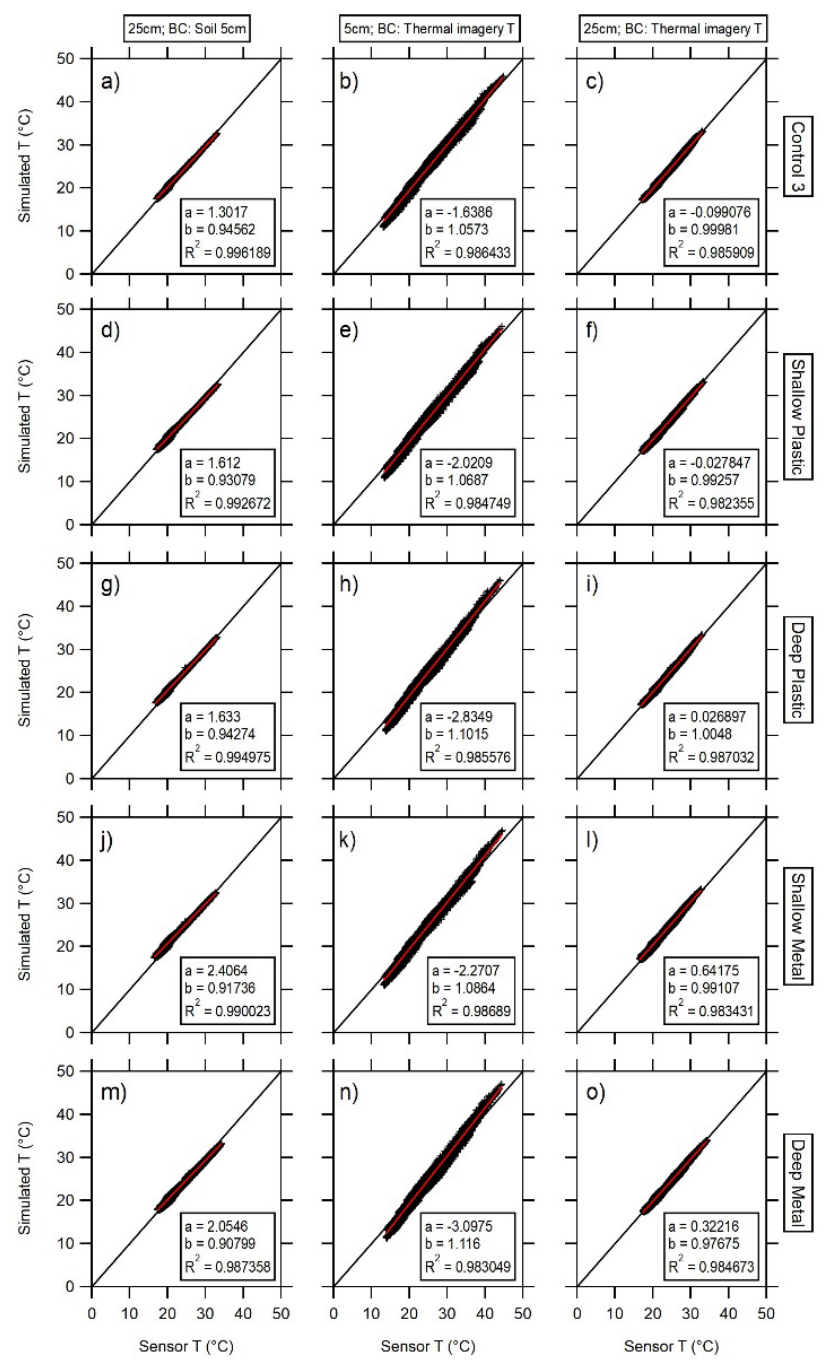

\subsubsection{Post-emplacement models}

For the post-emplacement models, C3, SP, DP, DM, and SM were simulated for a total time period of 94 days. Out of the five controls (see Figure 14), only one control was modeled (C3) because of similar temperatures and a low standard deviation in comparison to the other 
controls $\left(\mathrm{C}_{1}, \mathrm{C}_{2}, \mathrm{C}_{4}\right.$, and $\left.\mathrm{C}_{5}\right)$. The measured and simulated temperatures for six soil depths $(5,15,25,35,45$, and $55 \mathrm{~cm})$ for $\mathrm{C}_{3}$ are shown in Figure Figure 18. Simulated temperatures at all depths show a close fit to the measured temperatures. 
Figure 18. Simulated (boundary condition (BC) thermal imagery and soil temperature) and measured soil temperatures at C3 at soil depths (a) $5 \mathrm{~cm},(b) 15 \mathrm{~cm},(c) 25 \mathrm{~cm},(d) 35 \mathrm{~cm},(e)$ $45 \mathrm{~cm}$, and ( $f 55 \mathrm{~cm}$ for the post-placement period (14 July-15 October 2018).
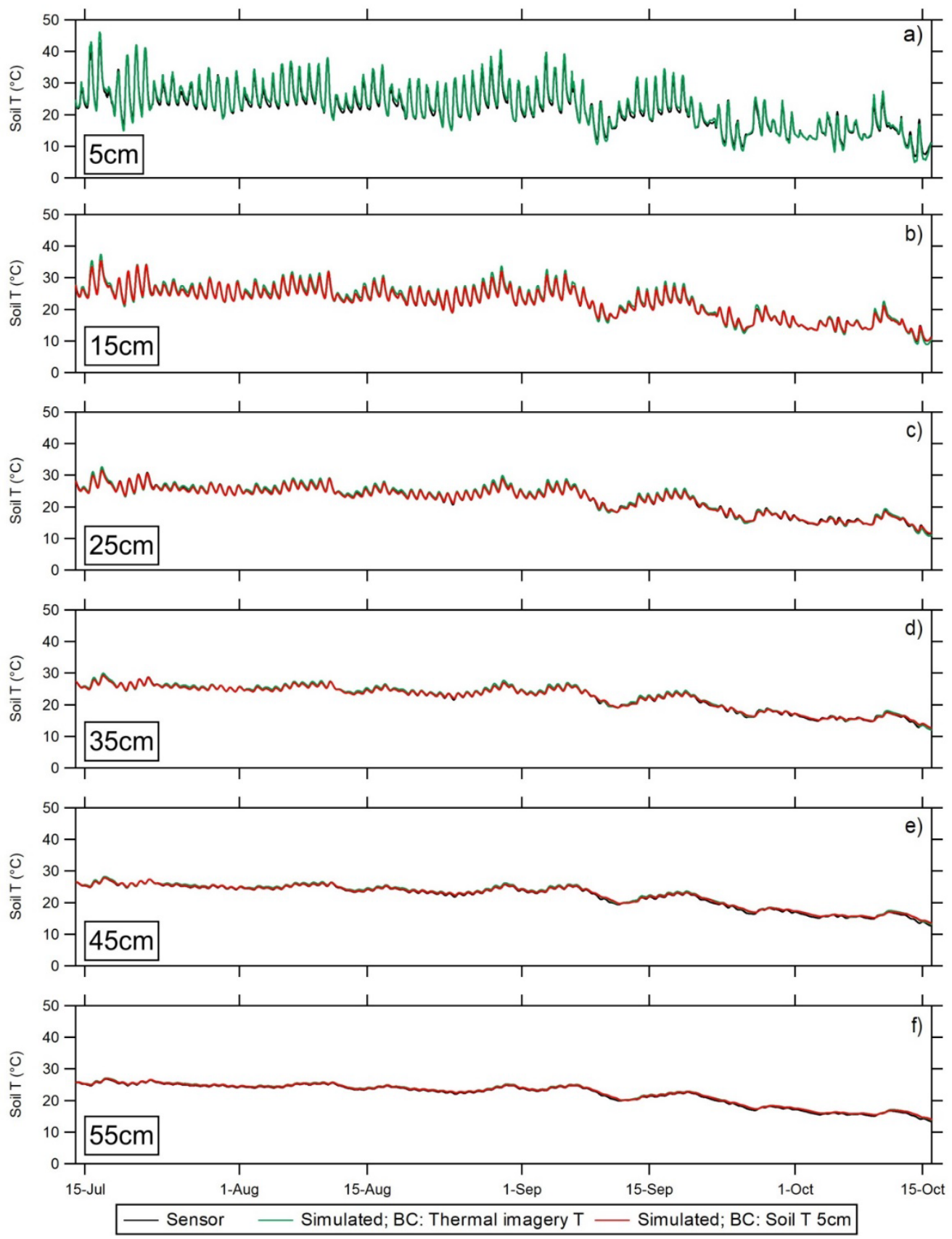

Regression graphs of 9,025 time-steps for the $\mathrm{C}_{3}$ simulations show a goodness of fit above 0.99 for both the calibration simulations and the simulations where a thermal imagery temperature was applied to the top boundary condition (Figure 19). A negative slope for the thermal imagery simulations at depths down to $35 \mathrm{~cm}$ indicate that the model slightly 
overestimates soil temperatures at higher temperatures and underestimates temperatures at lower temperatures.

Figure 19. Regression plots of sensor temperature and simulated soil temperature for different soil depths at C3 (top panels) $(y=a+b x)$. Temperature difference between the measured temperature (sensor) and model (bottom panel) at C3.


$\begin{array}{llllllllllllllllllllllllllllllllllll}0 & 10 & 20 & 30 & 40 & 50 & 0 & 10 & 20 & 30 & 40 & 50 & 0 & 10 & 20 & 30 & 40 & 50 & 0 & 10 & 20 & 30 & 40 & 50 & 0 & 10 & 20 & 30 & 40 & 50 & 0 & 10 & 20 & 30 & 40 & 50\end{array}$

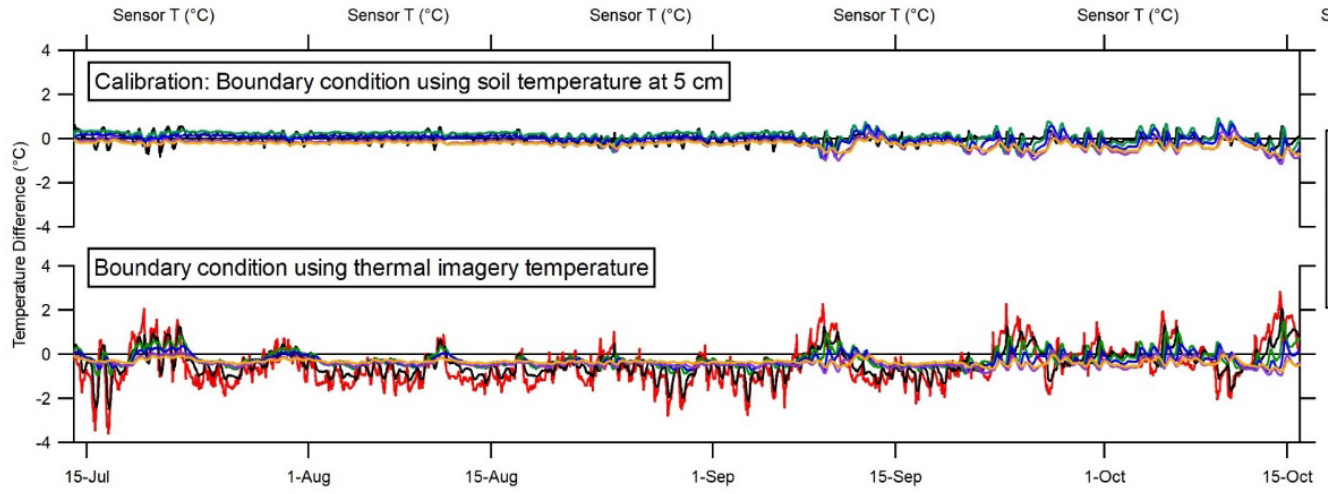

Sensor $\mathrm{T}\left({ }^{\circ} \mathrm{C}\right)$

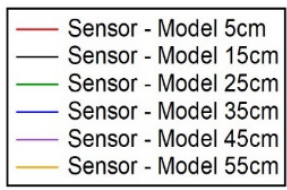


A comparison between the measured and simulated temperatures at $\mathrm{C}_{3}$ was also done by investigating the temperature difference between the measured and simulated temperature at several depths $(5,15,25,35,45$, and $55 \mathrm{~cm}$ ). The temperature difference between the measured and simulated temperature is lower for the simulations when the $5 \mathrm{~cm}$ sensor temperature is applied to the boundary condition compared to when the thermal imagery temperature is applied. The average temperature of the difference ranges from $-0.009^{\circ} \mathrm{C}$ to $-0.25^{\circ} \mathrm{C}$ for the $5 \mathrm{~cm}$ boundary condition compared to $-0.21^{\circ} \mathrm{C}$ to $-0.53^{\circ} \mathrm{C}$ for the thermal imagery temperature boundary condition. For the calibration simulations, the temperature difference ranges from a minimum of $-1.17^{\circ} \mathrm{C}$ to a maximum of $0.96^{\circ} \mathrm{C}$. The temperature difference is largest $\left(-3.63^{\circ} \mathrm{C}\right.$ to $\left.2.86^{\circ} \mathrm{C}\right)$ for the $5 \mathrm{~cm}$ thermal imagery simulation. The smallest temperature difference is at the $55 \mathrm{~cm}$ depth $\left(-0.8^{\circ} \mathrm{C}\right.$ to $\left.0.0075^{\circ} \mathrm{C}\right)$.

The measured and simulated soil temperatures at soil depths of $5 \mathrm{~cm}$ and $25 \mathrm{~cm}$ at each object (SP, DP, DM, and SM) are shown in Figure 20. Similar to the control simulations, the simulated temperatures show a close fit to the measured temperatures. 
Figure 20. Simulated (BC thermal imagery and soil temperature) and measured soil temperatures at $5 \mathrm{~cm}$ and $25 \mathrm{~cm}$ bgs at the (a) SP, (b) DP, (c) SM, and (d) DM for the postplacement time period (14 July-15 October 2018).
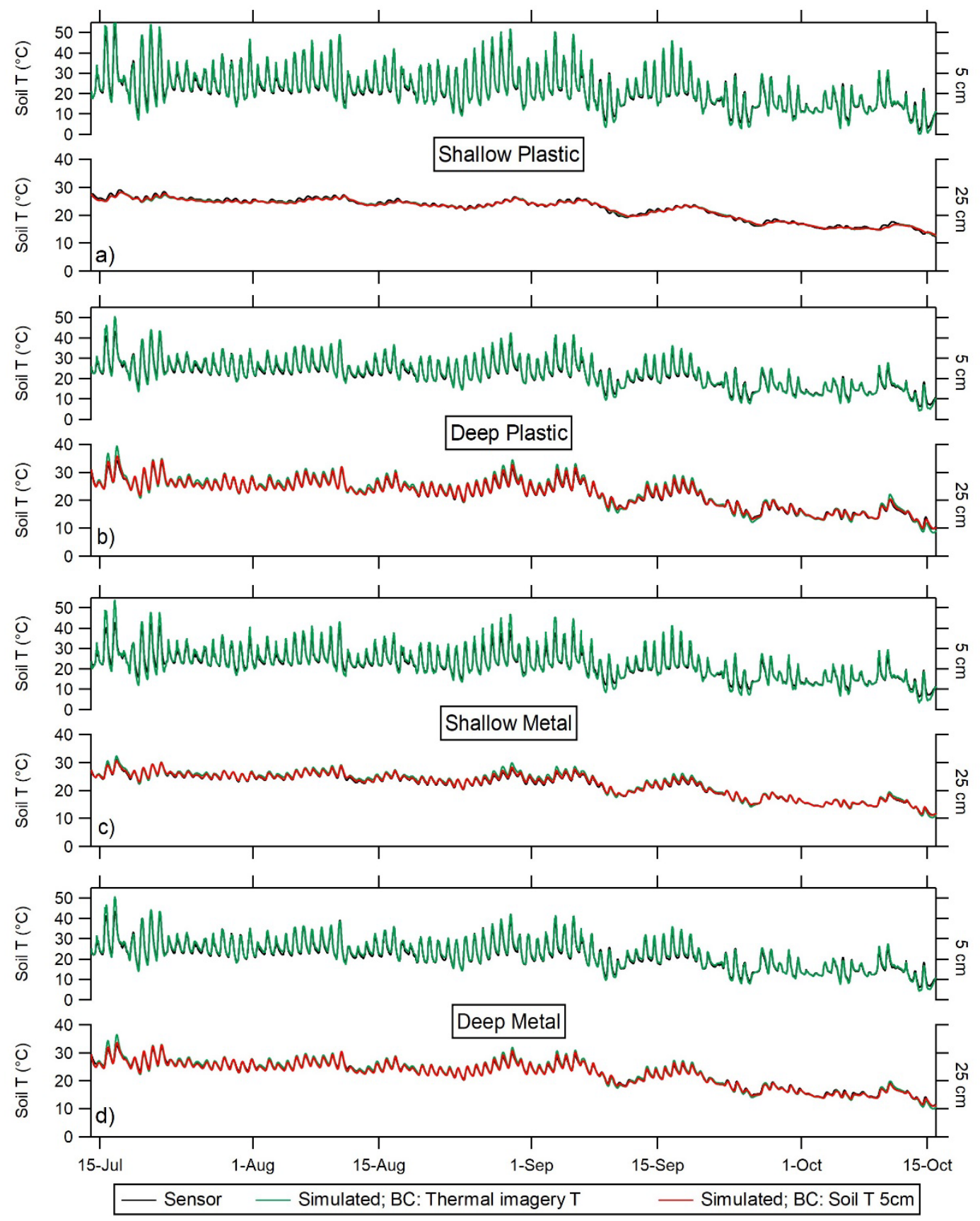

\subsection{Section 5 discussion}

A model of the pre- and post-emplacement of the buried objects for the field test in 2018 was calibrated using soil temperature measurements at a depth of $5 \mathrm{~cm}$. Additional calibration was performed by applying the thermal imagery temperatures as the surface boundary condition. Thermal 
imagery temperatures were averaged from nine pixels above each object (or from the center of each control square). It was shown that the standard deviation between the nine pixels was low, making the average suitable as a top boundary condition.

In general, simulated soil temperatures exhibit a good fit to measured temperatures when simulations utilized the $5 \mathrm{~cm}$ soil temperatures as a boundary condition. A poorer fit occurred when the thermal imagery temperature was used as the upper boundary condition. The better goodness of fit found in the models using the measured soil temperatures at a depth of $5 \mathrm{~cm}$ for a boundary condition suggests there is slight systematic error in the conversion from the thermal imagery radiance values to thermal imagery temperature. Additional simulations (section 3) were performed where thermal imagery radiance values were converted to thermal imagery temperatures and then corrected for the air temperature, relative humidity, and emissivity. When comparing the two modelsuncorrected thermal imagery surface temperatures versus corrected temperatures (air temperatures, relative humidity, and emissivity)-some time periods exhibited a better match with one model than the other. However, model performance varied depending on the time period selected. This result indicates more careful calibration of the thermal imagery conversion parameters are needed for future modeling efforts in order to better match surface soil temperatures. It is also extremely important to address and validate the radiance value conversion to temperature when using thermal imagery in any other analysis. Working with radiance values rather than converted thermal imagery temperatures is the preferred approach when performing any imagery and buried object analysis. Radiance value conversion of thermal IR imagery is only necessary when absolute temperatures are required, such as calibrating models or comparing with other sensor-derived temperature measurements. From an object identification perspective, conversion of thermal IR radiance values to temperatures is not needed. In this modeling effort, because these discrepancies, or offsets, are comparable between the models presented in this report, there is not a direct need to improve this offset to test the impact of environmental factors on object detectability with thermal IR. In conclusion, this model is well-calibrated and can be used to test the effects of different environmental factors to thermal signal response to buried objects. 


\section{Automated Detection of Buried Objects: A Simple Classifier Method}

The main objective of this subtask is to determine the effect of environmental phenomenological conditions on the ability to detect buried objects using thermal IR sensors.

\section{Problem Statement}

Our objective can be summarized concisely by Figure 21. The initial concern is the arrow $\phi$, which represents the environmental effects on the IR images. This is too broad a question, so we use the arrow $\psi$ to determine specific characteristics of the IR images that are of interest, which we represent with the object variables. After doing this, the problem moves from $\phi$ to the arrow $f$ mapping the environmental variables $X$ to the object variables $y$. The goal of mapping $f$ is to understand how the environmental variables are related to the computed object variables, which are used as representatives for the IR images.

Figure 21. A schematic of our main objectives.

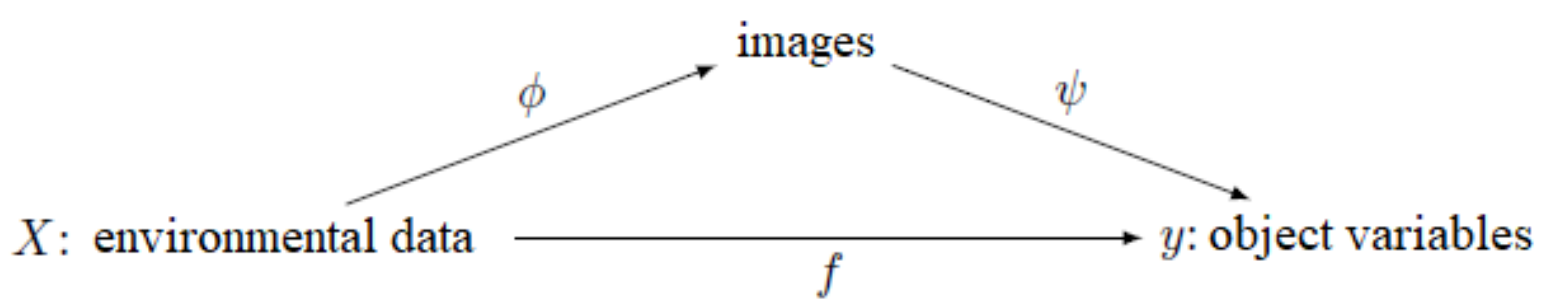

Section 6.3 is concerned with the arrow $\psi$ from Figure 21-that is, distilling the relevant IR image features down to one or more object variables. Section 6.4 is concerned with the study of $f$, in which the goal is to understand the complex relationship between the environmental variables and the object variables. We now organize the precise objectives of this work.

Objective 6.1. Define the mapping $\psi$ between the IR images and object variables such that the image features relevant to IR object detection are identified by the object variables. 
For the features relevant to detection we refer to Schacter (2012, 2018, 2020) and Ahuja and Schacter (1981), and ultimately in this work we focus simply on average absolute contrast, which we address in Section 6.3. Some evidence that this is a reasonable approach to this objective is presented in Section 6.3.1. Having addressed Objective 6.1 related to the images, we can now define the objectives related to the environment.

\section{Objective 6.2. Approximate a low-dimensional representation of the observations of $X$ and understand the relation between the (potentially latent) variables describing this representation and the object variables.}

Section 6.4.2 details an unsupervised approach to this objective. Section 6.4.3 uses a similar approach using supervised techniques. Both techniques introduce latent variables, which are variables that are not directly observed (i.e., the environmental variables) but are inferred through mathematical modeling. These latent variables are used to obtain a lower-dimensional approximation of the observed variables. This approximation is achieved by projecting $X$ to a latent space, $W$, which contains the learned latent variables, and involves studying the induced map from $W$ to the object variables, $y$. Objective 6.3 involves studying the map $f$ between the environmental variables and the object variables directly and is addressed in Section 6.4.4, Section 6.4.5, and Section 6.4.6.

Objective 6.3. Approximate a function between the environmental variables and the object variables, $f: X \rightarrow y$, and interpret the effects of the environmental variables such that:

1. The environmental variables with the largest effect on the object variables are identified

2. Environmental variable ranges in relation to object variables are understood

3. Interactions between the environmental variables in relation to object variables are understood

\subsection{Introduction}

This work is concerned with IR detection performance based on snapshots of data (assumed to be independent and identically distributed). The first main result is a method to measure IR detection performance by the 
performance of a detection algorithm in Section 6.3.1. This framework allows us to associate to each IR image a number in the unit interval $[0,1]$ that measures the overall performance of a detection algorithm applied to the IR image in terms of AUC of a ROC. These AUC values can then be used to measure the effect of environmental conditions on IR detection of objects.

To illustrate the use of these AUC distributions, consider the principle component analysis (PCA) results in Section 6.4.2. PCA is a dimension reduction technique in multivariate statistics in which the goal is to describe the higher-dimensional input environmental data with a lowdimensional set of latent variables in a way that preserves the statistical variance in the data as much as possible. In Figure 6.9 it appears that the AUC tends to be higher when the first PCA component is negative. To test this hypothesis we compare the baseline distribution of AUC values (using all IR images with buried targets) against the test distribution of AUC values where we only consider data based on some environmental condition (in this case the condition is that the first PCA component is negative). Figure 30 shows how the AUC distributions change from the baseline behavior with respect to the environmental change being tested. The plot on the left shows how this change affects the SM object. The plot on the right shows how this change affects all objects considered in the experiment. This visualization is summarized by two rows in Table 14. These AUC distribution tables are used to compare the AUC distributions across various types of buried objects. Each AUC distribution table summarizes nine different distribution plots as in Figure 30.

A more striking result using dimension reduction appears in Section 6.4. Here we use sliced inverse regression (SIR) to locate a subset of the data where all types of targets have AUC distributions with a mean above 0.92 (see Table 15). The targets with an AUC distribution mean above 0.92 are considered in this analysis because this value also identifies a cluster of observations highlighted through the k-means clustering method, indicating the importance of this cluster of observations. The downside to this result is that the size of the data subset is small ( 716 observations). Objective 6.2 is addressed through the use of both PCA and SIR in an effort to obtain a lower-dimensional approximation of the input variables to identify notable features and clusters. 
The main results of this work are obtained via two steps. First, through the construction of a nonlinear function that approximates the true relationship between environmental inputs and AUC outputs (Section 6.4.4). Second, through the interpretation of these input/output relationships (Sections 6.4.5 and 6.4.6). Together these two steps address Objective 6.3.

The second step involving the interpretation of the relationship between the environmental inputs and the AUC outputs (object variables) is accomplished through the use of two different analysis methods. First, we use Shapley values, which give a measure for how much each environmental variable contributed to the mapping between the environmental variables and the object variables represented by the nonlinear function constructed in the first step (Lundberg and Lee 2017; Lundeberg et al. 2018, 2020). In essence, Shapley values help us identify which environmental variables play an important role in the object identification task. Second, we use Sobol sensitivity analysis (Sobol 2001), which is a form of global sensitivity analysis. The idea behind the Sobol sensitivity analysis is to decompose the variance of the AUC output into fractions, each of which can be attributed to certain environmental inputs. Figure 35 (right) and Figure 37 (left) list the most important environmental variables affecting the data model according to the two interpretation methods, Shapleyvalues and Sobol sensitivity analysis, respectively. We first summarize the results based on the Shapley methods. From the approach taken in Section 6.4.5, we obtain first-order trends between environmental variables and AUC output. The sensitivity analysis carried out in Section 64.6 gives some understanding of the second order interaction effects between the environmental variables. Second-order Sobol indices (Sobol 2001) are used to understand the second-order interactions between the environmental variable with the highest first-order Sobol sensitivity and the other remaining environmental variables. We now turn our attention to the description of the environmental variables.

\subsection{Environmental data snapshot}

The environmental data prepared in this section begins with data collected during a study conducted in 2018 at the ERDC-CRREL facility detailed in (Clausen et al. 2020, 2021). This study monitored a homogenized patch of soil from May to November 2018 by taking buried sensor readings and monitoring weather conditions at 15-minute resolution. By snapshot we 
mean that the sequential aspect of the data as a time series is (mostly) disregarded.

We first subdivide the data based on the date and time of collection in relation to object emplacement. Data collected prior to object emplacement is discussed in Section 6.2.2. Data collected after target emplacement is discussed in Section 6.2.3.

Within each of these sections the data is further subdivided based on the variables being considered. When we combine all sensor data, weather measurements, and sun position variables, obtained using the methods described in (Kennedy 2020), we obtain over 300 environmental variables. In this work we analyze the complete set of all available variables as well as a subset of variables potentially more relevant to applications.

\subsubsection{Environmental variable description}

In this section we summarize the environmental variables monitored in (Clausen et al. 2021, 2020). There are approximately 300 environmental variables observed during this study. In the following we refer to Appendix $\mathrm{C}$ for a complete variable name explanation and a description of sensor locations and (Clausen et al. 2021, 2020) for more details.

In the test plot, the $\mathrm{CS} 655$ sensors measure the temperature $\left({ }^{\circ} \mathrm{C}\right)$, electric conductivity $(\mathrm{dS} / \mathrm{m})$, and soil moisture (\%). The sensors are buried at various depths within the test plot and the specific locations are shown in Appendix C. In the following analysis, for example in Figure 35, the depth and sensor location is listed in parenthesis for the corresponding environmental variable. Thermistors are used to monitor temperature of the soil $\left({ }^{\circ} \mathrm{C}\right)$ and they are also buried at various depths and locations throughout the test plot. We use the same convention in the following analysis to indicate the corresponding depth and sensor the considered variable.

Other environmental variables tracked for the study include heat flux $\left(\mathrm{W} / \mathrm{m}^{2}\right)$, heat plate temperatures $\left({ }^{\circ} \mathrm{C}\right)$, and incoming and reflected solar (short-wave) radiation $\left(\mathrm{W} / \mathrm{m}^{2}\right)$. Weather conditions are tracked using the ERDC-CRREL facility, and these measurements include average air temperature $\left({ }^{\circ} \mathrm{C}\right)$, average pressure (mbar), 24-hour rain accumulation total $(\mathrm{mm})$, relative humidity (\%), average wind speed $(\mathrm{m} / \mathrm{s})$, and average 
wind direction $\left({ }^{\circ}\right)$. Weather data are also collected from the nearby Lebanon airport, and this includes cloud cover (\%), pressure (mbar), oneand three-hour rain totals $(\mathrm{mm})$, one- and three-hour snow total ( $\mathrm{mm}$ ), and the minimum and maximum temperature for the day $\left({ }^{\circ} \mathrm{C}\right)$. Finally, sun position and time of day variables are computed using the methods in Kennedy (2020). These metrics include absolute time (ms), absolute time after previous dawn (ms), absolute time after previous dusk (ms), solar azimuth, solar elevation, solar zenith, and time of day in military time.

Both the full set of the 300 environmental variables and a chosen subset are used in the analysis. The subset consists of variables that are more likely to be measurable in the field and available through public sources and therefore potentially more applicable to field operations. This subset includes meteorological variables from the ERDC-CRREL test site as well as from the nearby Lebanon airport. The subset also includes solar radiation variables (incoming and reflectance) and shallow soil sensor variables down to $15 \mathrm{~cm}$ bgs. The thought is some subset of these variables can be estimated in the field, although determining the precise subset of variables applicable in the field requires further investigation.

\subsubsection{Before object emplacement}

Collecting data before object emplacement is important for characterizing the baseline behavior of sensors monitoring the test plot. The environmental data we refer to as before object emplacement occurred between 23 May 2018 at 1345, when data collection began, and 11 July 2018 at 0815 , when the test plot began to be disturbed by object emplacement activities. After filtering environmental observations to only include timestamps within 5 minutes of an IR image observation, we obtain 4,495 observations of environmental data before object emplacement.

As described in Section 6.2.1, two sets of data were prepared using the timestamps before object emplacement. One set includes all environmental variables, and one set includes a subset of environmental variables more relevant to applications.

\subsubsection{After object emplacement}

The environmental data we refer to as after object emplacement occurred between 13 July 2018 at 1445 (after disturbances involved with object 
emplacement ended) and 15 November 2018 at 0545 (when the last IR image was collected). After filtering environmental observations to only include timestamps within 5 minutes of an IR image observation, we obtain 11,705 observations of environmental data after object emplacement. As described in Section 6.2.1, two sets of data were prepared using the timestamps after object emplacement. One set includes all environmental variables, and one set includes a subset of environmental variables more relevant to applications.

Now that we have an understanding of the environmental variables used in the following modeling and analysis, we must turn our attention to the definition of the object variables. In the next section, Objective 6.1 is addressed through the definition of the object variables which are used as a representative of the IR images in the modeling.

\subsection{Object variables}

In this section we define the object variables from the schematic in Figure 21. The main focus in defining an appropriate object variable is to determine precisely what features of the IR image we want to study.

One possible object variable is a visibility index, defined in Clausen et al. (2021), where the response variable conveys a measurement of how visible the buried objects are to a human observer of the image. Another possibility-the one we will focus on in this work-is to replace the human observer with a classification algorithm and use the performance measurement of this algorithm as the response we want to study.

Notation and standard techniques for measuring classifier performance are discussed in Appendix B. Section B.1 is an introduction to binary classification algorithms. The performance of a binary classifier can be measured according to the ROC analysis, which is reviewed in Section B.2. This section focuses on the specific object variables used in this work.

\subsubsection{Simple classifier}

A simple classifier was constructed to capture the contrast between object and non-object pixels. To do this we use the known locations (in the frame of reference determined by IR camera pixels) of buried objects in the area of interest (AOI) using object locations from Clausen et al. (2020). 
We begin by subdividing the AOI as shown in Figure 22 and sampling from various pixel regions of the image. The AOI has four buried objects, which we label SP, DP, SM, and DM. The pixel coordinates used to sample over the targets are drawn in maroon. Control regions without targets are drawn in cyan. The background region without targets is drawn in green.

Figure 22. The AOI with buried object locations with respect to the frame of reference defined by the IR image pixels.

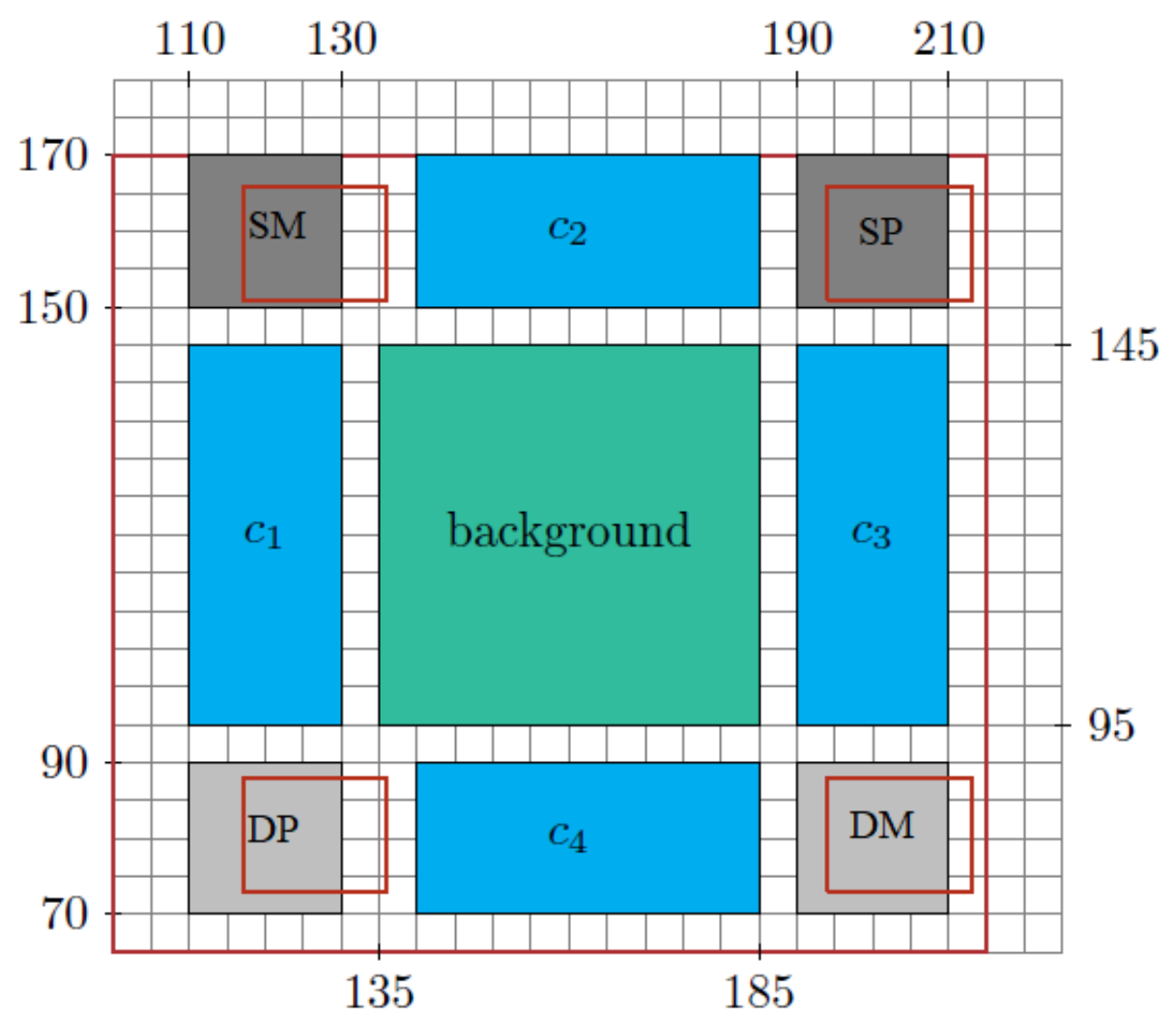

To build a simple classifier we compare pixels over objects and in control regions with the background pixels. To do this we sample $k_{0} \times k_{0}$ submatrices from the controls, background, and adjusted object locations in Figure 22. Let $k_{1}$ be the number of submatrices sampled from each object location and from each control. This gives us $8 k_{1}$ submatrices with $k_{1}$ submatrices from each of the 8-pixel regions (four objects and four controls).

Let $A_{i}$ denote one of the $8 k_{1}$ submatrices of size $k_{0} \times k_{0}$, let $\alpha_{i}$ be the average pixel value of $A_{i}$, let $k_{2}$ be the number of $k_{0} \times k_{0}$, submatrices sampled from the background pixels, and let $B_{j}$ denote one of the background submatrices with average pixel value $\beta_{j}$. We now compare the 
contrasts which is defined to be the difference between the average pixels and the average background pixels $\left|\alpha_{i}-\beta_{j}\right|$ for indices in the following range:

$$
\begin{aligned}
& 1 \leq i \leq 8 k_{1} \\
& 1 \leq j \leq k_{2}
\end{aligned}
$$

to construct a scoring function as defined in Section B.1.

Using the notation from B.1, an input $u$ is one of the random $8 k_{1}$ submatrices $A_{i}$. The ground truth $g$ is determined by the sampling location of $A_{i}$ in the image. More precisely, the ground truth is defined as:

$$
\boldsymbol{g}\left(\boldsymbol{A}_{\boldsymbol{i}}\right)=\left\{\begin{array}{l}
\text { no, if } \boldsymbol{A}_{\boldsymbol{i}} \text { is in control region } \\
\text { yes, if } \boldsymbol{A}_{\boldsymbol{i}} \text { is in target region }
\end{array} .\right.
$$

The scoring function $f$ on the input $A_{i}$ is defined by taking the mean of the absolute value of the contrasts $\left|\alpha_{i}-\beta_{j}\right|$ over all $j$,

$$
f\left(A_{i}\right):=\frac{1}{k_{2}} \sum_{j=1}^{k_{2}}\left|\alpha_{i}-\beta_{j}\right| .
$$

The above scoring function will be used to measure classifier performance to obtain the desired object variable. For the remainder of this section we let $k_{0}=5, k_{1}=30$, and $k_{2}=100$. Section B.2 explains how to measure the performance of the resulting classifier using the area under the ROC curve (AUC) from a list of scores with their corresponding ground truth labels.

Next, we describe precisely how to produce the lists of scores given this classifier setup using an explicit example. Suppose we are interested in the performance (in terms of AUC) of the binary classifier in detecting the DP object. Then we take the $k_{1}=30$ matrices (of size $k_{0} \times k_{0}$ with $k_{0}=5$ ) and compute their score using Equation (14). This yields $k_{1}=30$ scores with ground truth label yes. The no labels are obtained from computing scores for the $4 k_{1}=120$ submatrices from the control regions. In this example we obtain $k_{1}$ yes scores (from $k_{0} \times k_{0}$ matrices over the DP object) and $4 k_{1}$ no scores (from the $k_{0} \times k_{0}$ matrices over the four controls). AUC is then computed using the machinery developed in Section B.2.

Suppose instead we are interested in the classifier performance in detecting any shallow object (plastic or metal). Everything stays the same 
except that there are now $2 k_{1}$ scores with yes labels $\left(k_{1}\right.$ for each of the two shallow objects). The no scores are the same as in the previous example. We can apply the above procedure to each IR image to obtain AUC values for each of the four objects (SP, SM, DP, and DM) as well as a grouping of plastic and metal objects, deep and shallow emplacements, and all four objects.

Lastly, since our classifier is probabilistic, we calculate mean values for the AUC to gain some statistical robustness (we computed each AUC value 50 times). The upshot is that for each IR image we compute nine AUC values (one for each group of objects) that measure the performance of the binary classifier described in this section on the given image and subset of objects.

\subsection{Data analysis}

This section is composed of techniques for analyzing data that is assumed to be independently and identically distributed, which we refer to as snapshot data. For details on the preparation of the input environmental data see Section 6.2. For details on the preparation of the object variables see Section 6.3 .

The objectives in Section 6.1 require a way to measure how target variables change as we place conditions on the input environmental variables. The approach we take to measure this change is based on comparing empirical distributions of the object variables. We begin by describing this approach (Section 6.4.1) and then use it in the subsequent sections.

\subsubsection{Simple classifier AUC distributions}

We now describe our approach for comparing empirical distributions of object variable values to measure differences based on the input environmental conditions. The object variables of consideration are defined in Section 6.3.1.

Recall from Section 6.3.1 that each IR image corresponds to nine AUC values between $\mathrm{O}$ and 1 based on the objects of interest. Probability density functions (pdfs) for these distributions can be approximated using Scott (2015) and Silverman (1982). To measure the empirical difference between two distributions, we report the calculated mean (indicated by $\mu$ ) and standard deviation (indicated by $\sigma$ ) values. We also report the 
Wasserstein distance, which is used to measure the distance between distributions (see Wasserman 2020 for additional information).

To introduce how we will use these concepts, we begin with an example comparing the AUC distributions before and after object emplacement. Figure 23 shows AUC distributions for all target variables before and after object emplacement.

Figure 23. AUC distributions (a) before target emplacement and (b) after target emplacement.
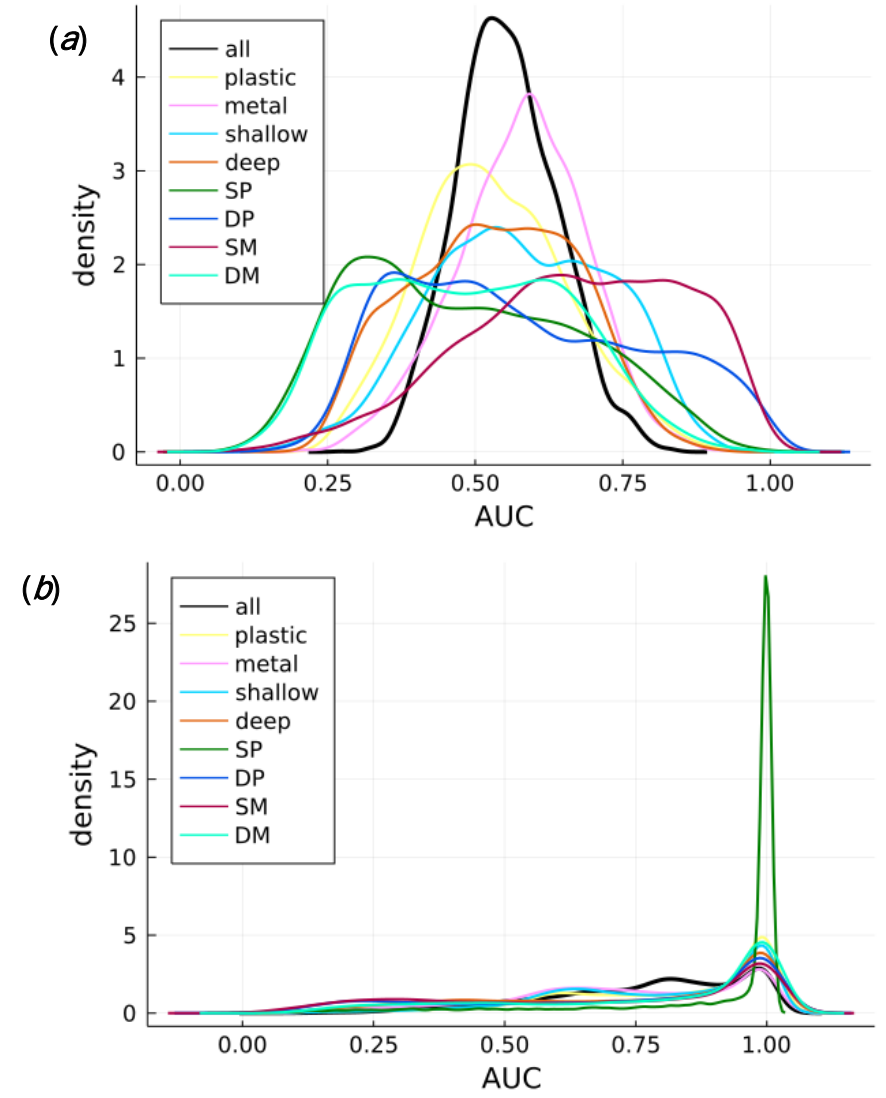

Figure 23 makes it visually clear that object emplacement has an effect on the AUC distributions (which is expected), but we want to further analyze these differences by comparing each pair of distributions individually.

Figure 24 compares AUC distributions computed using all objects and the Wasserstein distance between the before and after emplacement distributions is 0.2191. Figure 25a shows the comparison between metal objects with a Wasserstein distance between the distributions of 0.16657 and Figure 25b shows a comparison of plastic objects with Wasserstein distance between the distributions of 0.2758 . Figure 26 includes two comparisons, one for deep objects in plot (a) and one for shallow objects. Figure 27 includes four comparisons, one for each individual object. Table 
13 distills all this information down to a single table of values. Since we will be using these tables (which we will refer to as AUC distribution tables) extensively, we conclude this section with an explanation of these values.

Figure 24. AUC distributions before and after target emplacement with AUC values computed using all targets. The Wasserstein distance between the distributions is 0.2191.

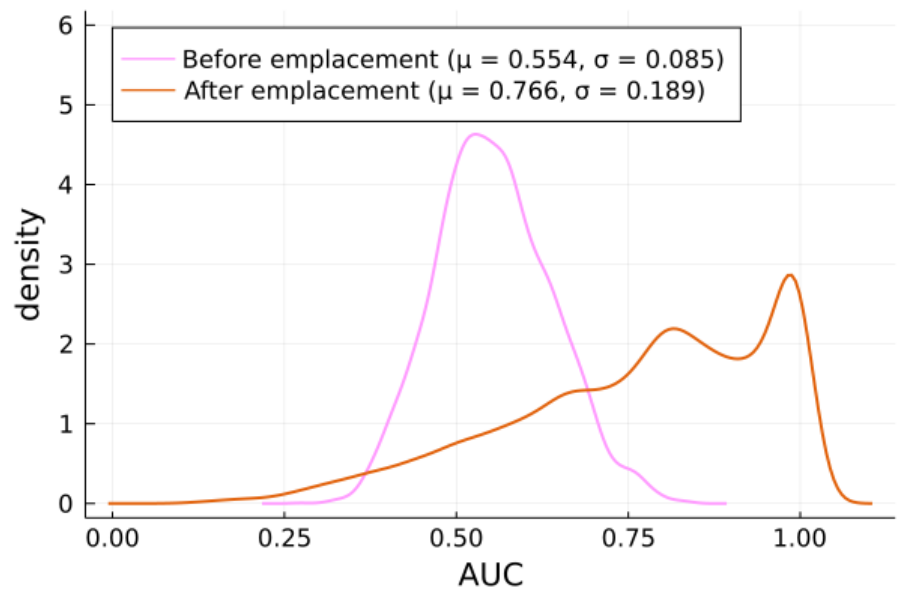

Figure 25. AUC distributions before and after object emplacement with AUC values computed using (a) metal objects with Wasserstein distance 0.1667 and $(b)$ plastic objects with Wasserstein distance 0.2758 .






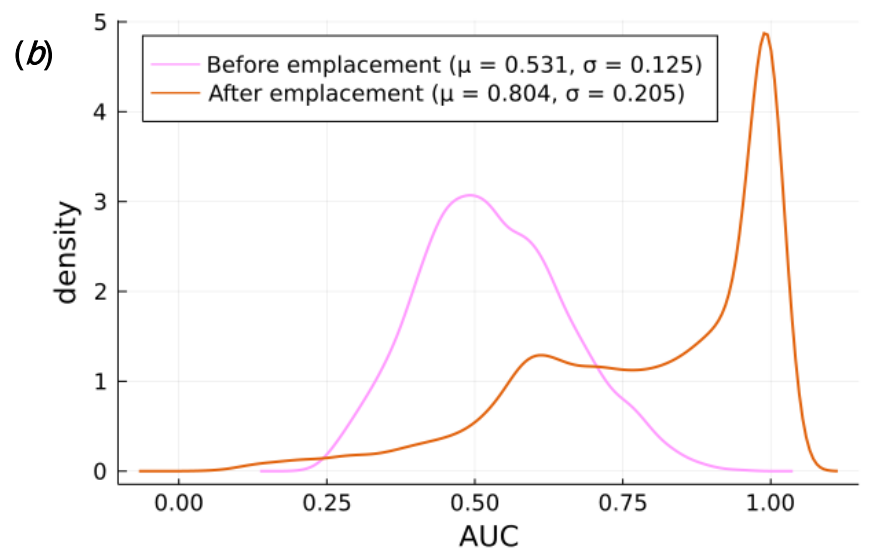

Figure 26. AUC distributions before and after target emplacement with AUC values computed using $(a)$ deep objects with Wasserstein distance 0.2226 and $(b)$ shallow objects with Wasserstein distance 0.2123 .


In Table 13 we are comparing AUC distributions for a baseline condition with a test condition. In this example, the baseline (or base) is before objects are buried. \#base is the number of data observations of the baseline condition and \#test is the number of data observations of the test condition. For Table 13, \#base is 4,495, which is the number of IR images 
taken before object emplacement and \#test is 11,705, which is the number of IR images taken after object emplacement.

For a given set of baseline and test observations (in this example it is before and after emplacement) there are nine pairs of AUC distributions to compare and correspond to the rows of Table 13. The mean values of base and test distributions are reported in the $\mu_{\text {base }}$ and $\mu_{\text {test }}$ columns, respectively. The standard deviations of the test and base sets are reported in the $\sigma_{\text {base }}$ and $\sigma_{\text {test }}$ columns. The column $\mu_{\text {change is defined by }}$ the difference between the mean of the test and the mean of the base,

$$
\mu_{\text {change }}=\mu_{\text {test }}-\mu_{\text {base }} \text {. }
$$

Finally, the last column reports the Wasserstein distance to compare the baseline distributions to the test distributions.

Comparing a test distribution to a baseline distribution is motivated by attempting to understand the underlying behavior against the test behavior. In the analysis that follows we will exclusively use the set of observations after target emplacement as the baseline behavior to test against. Comparing the effect of environmental conditions against this baseline behavior is the focus of these analysis sections.

Figure 27. AUC distributions before and after object emplacement with AUC values computed using individual objects. The comparisons are for the (a) SP object with Wasserstein distance

0.4088, (b) DP object with Wasserstein distance 0.1704, (c) SM object with Wasserstein distance 0.1221 , and () $d \mathrm{DM}$ object with Wasserstein distance 0.2879 .

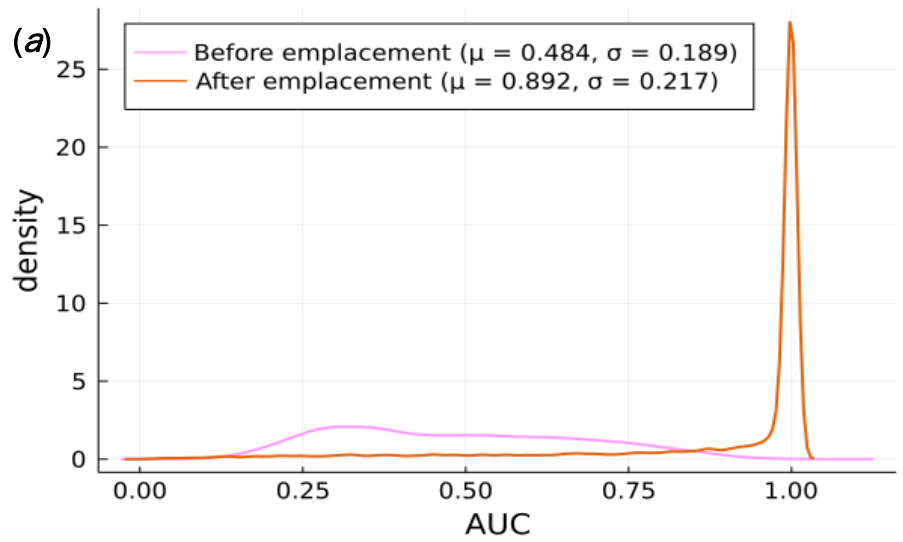



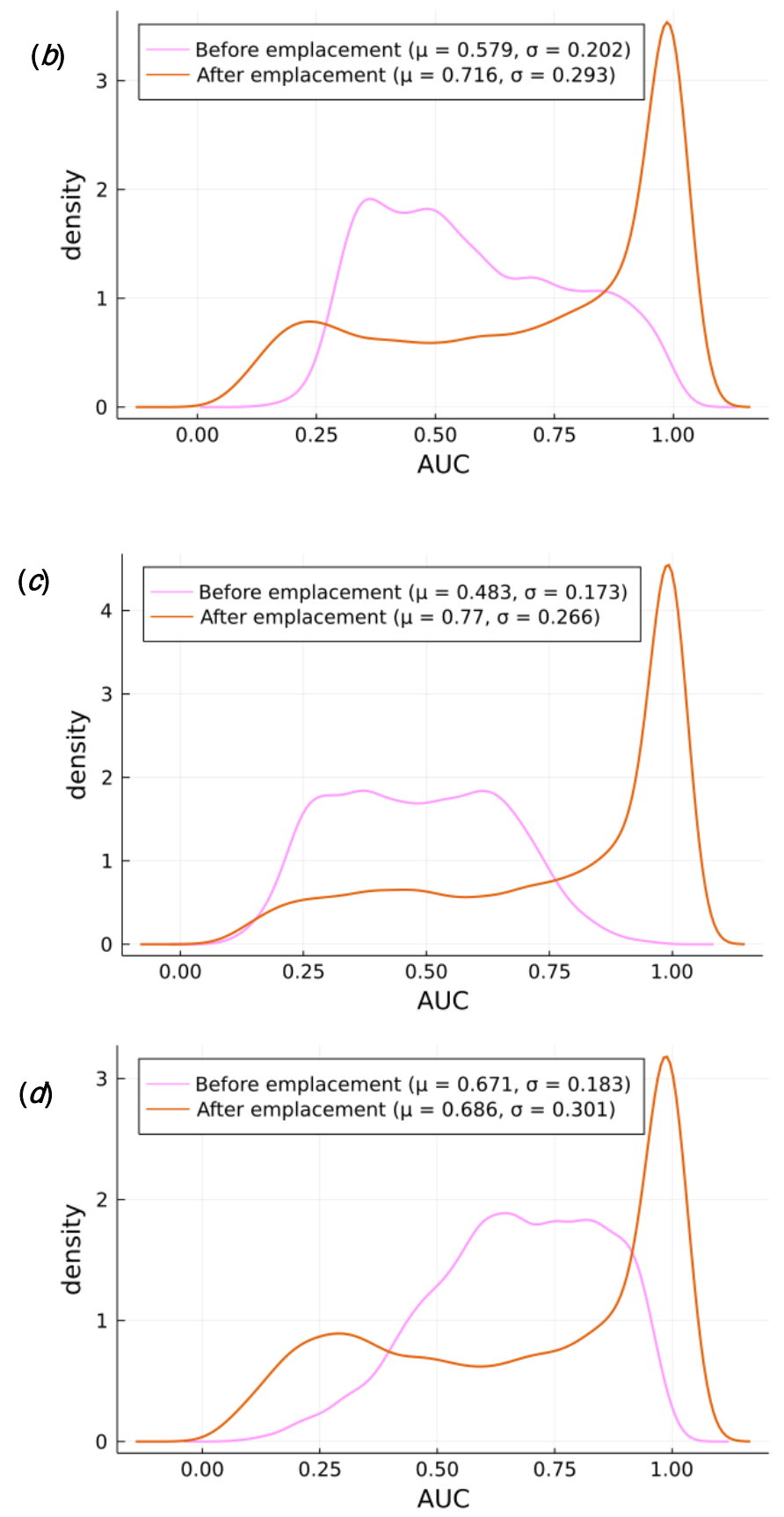

Table 13. AUC distribution table with baseline before target emplacement and test after object emplacement.

\begin{tabular}{|l|r|r|r|r|r|r|}
\hline targets & \multicolumn{1}{c|}{$\boldsymbol{\mu}_{\text {change }}$} & \multicolumn{1}{c|}{$\boldsymbol{\mu}_{\text {base }}$} & \multicolumn{1}{c|}{$\boldsymbol{\mu}_{\text {test }}$} & \multicolumn{1}{c|}{$\boldsymbol{\sigma}_{\text {base }}$} & \multicolumn{1}{c|}{$\boldsymbol{\sigma}_{\text {test }}$} & Wasserstein \\
\hline all & 0.212 & 0.554 & 0.766 & 0.085 & 0.189 & 0.2191 \\
\hline SP & 0.407 & 0.484 & 0.892 & 0.189 & 0.217 & 0.4088 \\
\hline DP & 0.137 & 0.579 & 0.716 & 0.202 & 0.293 & 0.1704 \\
\hline DM & 0.287 & 0.483 & 0.77 & 0.173 & 0.266 & 0.2879 \\
\hline SM & 0.015 & 0.671 & 0.686 & 0.183 & 0.301 & 0.1221 \\
\hline
\end{tabular}




\begin{tabular}{|l|r|r|r|r|r|r|}
\hline targets & \multicolumn{1}{c|}{$\boldsymbol{\mu}_{\text {change }}$} & \multicolumn{1}{c|}{$\boldsymbol{\mu}_{\text {base }}$} & \multicolumn{1}{c|}{$\boldsymbol{\mu}_{\text {test }}$} & \multicolumn{1}{c|}{$\boldsymbol{\sigma}_{\text {base }}$} & \multicolumn{1}{c|}{$\boldsymbol{\sigma}_{\text {test }}$} & Wasserstein \\
\hline metal & 0.151 & 0.577 & 0.728 & 0.11 & 0.218 & 0.1657 \\
\hline plastic & 0.272 & 0.531 & 0.804 & 0.125 & 0.205 & 0.2758 \\
\hline deep & 0.212 & 0.531 & 0.743 & 0.138 & 0.261 & 0.2226 \\
\hline shallow & 0.211 & 0.578 & 0.789 & 0.149 & 0.202 & 0.2123 \\
\hline
\end{tabular}

\subsubsection{Principal component analysis}

Principal component analysis (PCA) is an unsupervised dimension reduction technique that aims to describe the input environmental data with a handful of latent variables. Dimension reduction techniques project data to spaces with lower dimensions while maintaining certain aspects of the data. In the case of PCA, the projection is an orthogonal linear map constructed to preserve as much statistical variance in the data as possible.

If we take the input environmental data prepared in Section 6.2 using all 300 environmental variables, then we can use PCA to project down to a dimension 9 space while maintaining over $95 \%$ of the variability in the data (Figure 28). Once the PCA projection has been computed using Bloam et al. (2020), we can begin to explore how the object variables behave under this mapping.

Figure 28. The explained variance as the number of PCA components increases when the PCA is computed using all environmental variables.

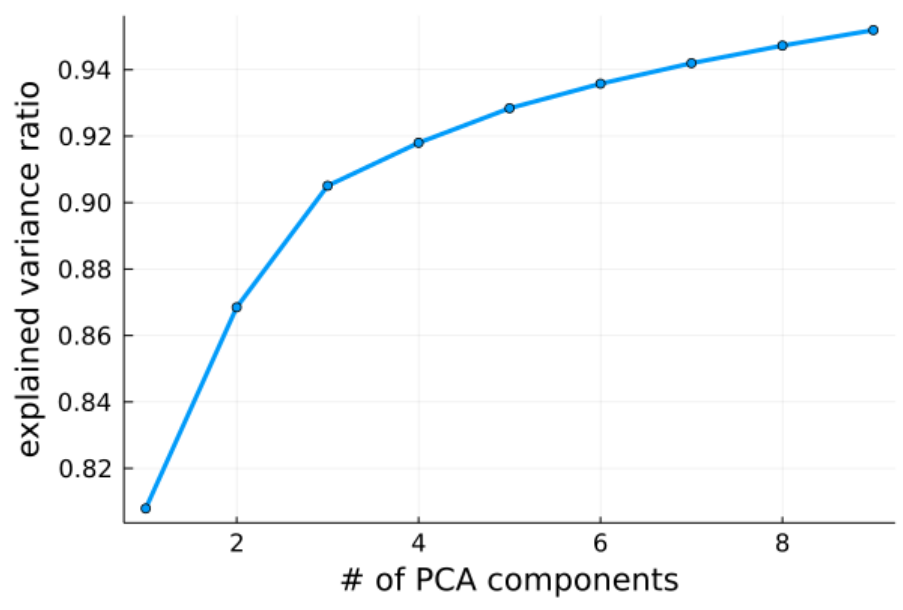

Since the object variables did not play a role in the PCA projection (it is an unsupervised method), we use coloring of the projected points to see if there is a relationship with the object variables (see Figure 29). Unfortunately, neither of the plots in Figure 29 appear to show any 
striking behavior. The visualizations do suggest there is potentially some separation based on the sign of the first PCA component.

Figure 29. Scatter plot of the first PCA component against (a) AUC and (b) scatter plot using three PCAcomponents with both plots colored byAUCusingall objects.


To analyze the scenario above we use the comparison techniques described in Section 6.4.1. For the baseline observations we now use all observations after object emplacement, which corresponds to the full set of 11,705 IR images after emplacement. For the test observations we use only those observations whose projection to the first PCA component is negative, and this reduces the test set to a subset of 6,900 IR images. Table 14 summarizes the results for the comparison for the situation when the first PCA component is negative. 
Table 14. AUC distribution for the baseline of all observations made after object emplacement against observations where the first PCA component is negative.

\begin{tabular}{|l|r|r|r|c|r|r|}
\hline targets & \multicolumn{1}{|c|}{$\boldsymbol{\mu}_{\text {change }}$} & \multicolumn{1}{c|}{$\boldsymbol{\mu}_{\text {base }}$} & \multicolumn{1}{c|}{$\boldsymbol{\mu}_{\text {test }}$} & \multicolumn{1}{c|}{$\boldsymbol{\sigma}_{\text {base }}$} & \multicolumn{1}{c|}{$\boldsymbol{\sigma}_{\text {test }}$} & \multicolumn{1}{l|}{ Wasserstein } \\
\hline all & 0.04 & 0.766 & 0.805 & 0.189 & 0.191 & 0.0395 \\
\hline SP & 0.005 & 0.892 & 0.897 & 0.217 & 0.204 & 0.0059 \\
\hline DP & 0.032 & 0.716 & 0.748 & 0.293 & 0.273 & 0.0318 \\
\hline DM & 0.002 & 0.77 & 0.771 & 0.266 & 0.27 & 0.008 \\
\hline SM & 0.12 & 0.686 & 0.806 & 0.301 & 0.26 & 0.1198 \\
\hline metal & 0.061 & 0.728 & 0.789 & 0.218 & 0.224 & 0.0607 \\
\hline plastic & 0.018 & 0.804 & 0.822 & 0.205 & 0.193 & 0.0184 \\
\hline deep & 0.017 & 0.743 & 0.759 & 0.261 & 0.256 & 0.017 \\
\hline shallow & 0.062 & 0.789 & 0.851 & 0.202 & 0.19 & 0.0624 \\
\hline
\end{tabular}

From Table 14 we see that this comparison highlights the SM object the most. To continue with this analysis we plot the SM comparison in Figure $30 a$ as well as the same plot using all targets for comparison in Figure $30 b$. Although PCA is a start toward Objective 6.2, we move on to supervised dimension reduction techniques (using the object variables) to tackle this objective.

Figure 30. AUC distribution comparisons (using observations whose first PCA component is negative) for (a) an SM object with Wasserstein distance of 0.1198 and $(b)$ all objects with Wasserstein distance of 0.0395 .

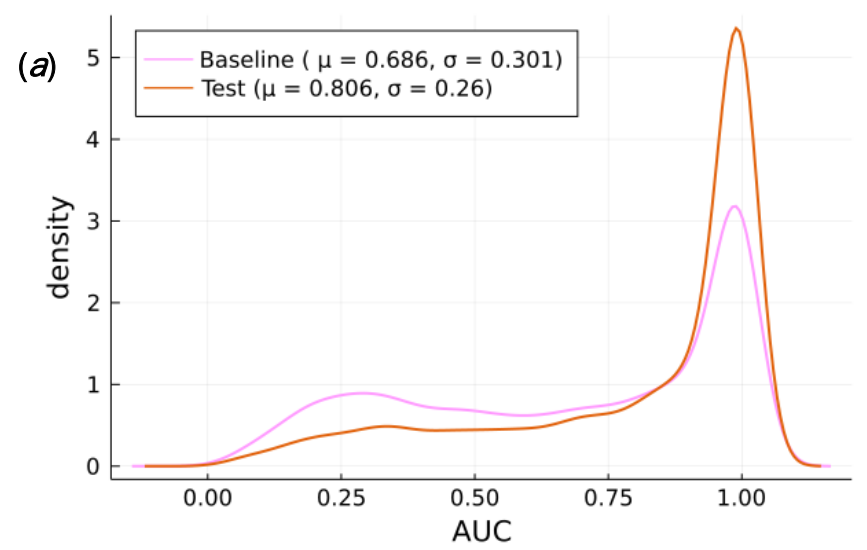


(b)

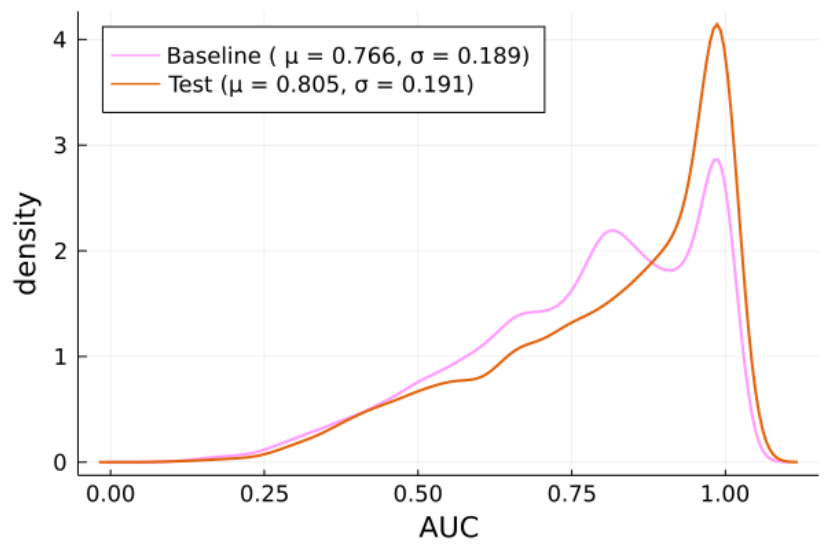

\subsubsection{Sliced inverse regression}

Sliced inverse regression (SIR) is a supervised dimension reduction technique proposed in $\mathrm{Li}$ (1991) and implemented in R Core Team (2020) and Weisberg (2002) alongside other algorithms based on this idea. Let $y$ denote the AUC target variable defined in Section 6.3 with sampling based on all buried objects. Then $y$ is univariate (instead of being composed of nine variables). Let $X$ be the approximately 300 environmental variables from Section 6.2.3. Inverse regression means that we regress $X$ against $y$ instead of regressing $y$ against $X$. Sliced inverse regression is an efficient and relatively simple technique that also avoids any type of predictive model fitting discussed in other sections. The algorithm produces eigenvectors, $w_{i}$, ordered by corresponding eigenvalue and we project the environmental variables $X$ to the latent space $W$ to get an $l$-dimensional representation of our data. The latent space is defined as the span over the $l$ eigenvectors produced by the SIR algorithm:

$$
W_{l}:=\operatorname{span}\left\{w_{1}, w_{2}, \ldots, w_{l}\right\}
$$

In Figure 31a we show the projection of the input data $X$ to three dimensions using $W_{3}$ with the color corresponding to the AUC object variable $y$. The most striking feature in this figure is a single high AUC cluster in the foreground of the plot. This cluster can be identified using the k-means algorithm (PyDataBlog 2020). After some experimentation with the value of $\mathrm{k}$, we can identify this high AUC cluster using k-means with $\mathrm{k}=7$. The high AUC cluster is approximated by cluster 7 from the $\mathrm{k}$ means clustering shown in Figure 31b. 
Figure 31. Environmental data $X$ projected to the SIR latent space $W_{3}$ from Equation (16) (a) colored by AUC and (b) via $k$-means with $k=7$.


To analyze this more closely we consider the two-dimensional latent space $W_{2}$ instead of $W_{3}$ shown in Figure 32. On the left we identify the high AUC cluster as the rectangular area bounded by the four cutoff values drawn in blue. On the right is the same projection colored using $k$-means assignments with $k=7$. Again, we can see that the high AUC cluster is identified by $k$-means clustering. 
Figure 32. Environmental data $X$ projected to the SIR latent space $W_{2}$ from Equation (16) (a) colored by AUC and $(b)$ via $k$-means with $k=7$.



To analyze the effect of this cluster on the AUC distributions we include an AUC distribution table (defined in Section 6.4.1) in Table 15. The identified subset consisting of 716 IR images within the blue rectangle in Figure $32 a$ form the test set for this comparison, and the baseline set is taken to be all 11,705 IR images after emplacement. As we can see from this table, we have identified a set of 716 observations based on the SIR latent variables $w_{1}$ and $w_{2}$ that dramatically impact the AUC distribution. The baseline distribution means ( $\mu_{\text {base }}$ in Table 15) range from 0.686 to 0.892 across subsets of objects, and the test distribution means ( $\mu_{\text {test }}$ in Table 15$)$ range from 0.927 to 0.961 for the 716 element subset of observations identified using SIR. Although the size of the test set of observations is relatively small, this analysis reveals a complicated relationship between the latent variables $w_{1}$ and $w_{2}$ and the AUC object variables in the direction of Objective 6.2 . 
Table 15. AUC distribution table comparing the baseline of all observations made after object emplacement against observations bounded by the blue cutoff values in Figure $6.32 a$.

\begin{tabular}{|l|r|r|r|r|r|r|}
\hline targets & \multicolumn{1}{c|}{$\boldsymbol{\mu}_{\text {change }}$} & \multicolumn{1}{c|}{$\boldsymbol{\mu}_{\text {base }}$} & \multicolumn{1}{c|}{$\boldsymbol{\mu}_{\text {test }}$} & \multicolumn{1}{c|}{$\boldsymbol{\sigma}_{\text {base }}$} & \multicolumn{1}{c|}{$\boldsymbol{\sigma}_{\text {test }}$} & Wasserstein \\
\hline all & 0.176 & 0.766 & 0.942 & 0.189 & 0.158 & 0.1763 \\
\hline SP & 0.07 & 0.892 & 0.961 & 0.217 & 0.146 & 0.0698 \\
\hline DP & 0.204 & 0.716 & 0.92 & 0.293 & 0.206 & 0.2039 \\
\hline DM & 0.165 & 0.77 & 0.935 & 0.266 & 0.193 & 0.1654 \\
\hline SM & 0.266 & 0.686 & 0.952 & 0.301 & 0.147 & 0.2659 \\
\hline metastic & 0.216 & 0.728 & 0.944 & 0.218 & 0.16 & 0.2156 \\
\hline deep & 0.137 & 0.804 & 0.941 & 0.205 & 0.164 & 0.1369 \\
\hline shallow & 0.185 & 0.743 & 0.927 & 0.261 & 0.196 & 0.1846 \\
\hline
\end{tabular}

\subsubsection{Nonlinear predictive models}

We now describe techniques to approximate $f$ in Figure 21, which represents the relationship between the environmental variables and the AUC object variables. The approximations described in Section 6.4.5 and Section 6.4.6 are used to address Objective 6.3. We follow the convention that $X$ is a matrix with a column for each environmental input variable and a row for each observed sample of these variables. Similarly, $y$ is a matrix with a column for each object variable of interest and a row of values for each row of $X$. Mathematically, the function $f$ maps the rows of $X$ to the rows of $y$.

The general approach used here requires splitting the input environmental data $X$ and the output object variable data $y$ into so-called training and testing subsets. The machine learning algorithm implemented requires a set of data to learn the mapping between the environmental variables and the object variables, this is the training set. A separate set of data called the testing set is required to assess how well the algorithm performed the task of learning the mapping between the environmental variables and the object variables. To identify the training and testing subsets we begin by choosing a random permutation of the indices that index the pairs of input environmental observations $X$ and output object observations $y$. Let $I$ denote these row indices, so that the randomly permuted data consists of pairs $\left(X_{i}, y_{i}\right)_{i \in I}$ where $X_{i}$ and $y_{i}$ denotes the $i$ th row of $X$ and $y$, respectively.

Next, we partition $I$ into two sets $I_{\text {train }}$ and $I_{\text {test }}$ such that we have the proportions: 


$$
0.7=\frac{\# I_{\text {train }}}{\# I} \text {, and } 0.3=\frac{\# I_{\text {test }}}{\# I} .
$$

Let $X_{\text {train }}$ and $y_{\text {train }}$ denote the inputs and outputs corresponding to the indices in $I_{\text {train }}$, we call these sets the training data. Let $X_{\text {test }}$ and $y_{\text {test }}$ denote the inputs and outputs corresponding to $I_{\text {test }}$, the test data. More precisely, the training data are the collection:

$$
\left\{\left(X_{i}, y_{i}\right)\right\}_{i \in I_{\text {train }}}
$$

and the test data are:

$$
\left\{\left(X_{i}, y_{i}\right)\right\}_{i \in I_{\text {test }}}
$$

Approximations to the map $f$ representing the relationship between the environmental variables and the object variables are obtained by solving an optimization problem using the training data.

We use the notation $\hat{f}$ to denote an approximation to $f$ and also use the notation:

$$
\widehat{y_{l}}:=\hat{f}\left(X_{i}\right) \text {, }
$$

to denote the predicted object variable given by evaluating the approximated function $\hat{f}$ at the ith row of the environmental variables. For an individual prediction $\widehat{y}_{l}$ we define the residual of that prediction by $\widehat{y}_{l}-$ $y_{i}=\hat{f}\left(X_{i}\right)-f\left(X_{i}\right)$, which is the difference between the predicted output and the actual output. The training error is defined using root mean square error (RMSE) on the training data.

$$
\operatorname{RMSE}_{\text {train }}:=\left(\frac{1}{\# I_{\text {train }}} \sum_{i \in I_{\text {train }}}\left(\hat{f}\left(X_{i}\right)-f\left(X_{i}\right)\right)^{2}\right)^{\frac{1}{2}}
$$

Similarly, the test error is defined by:

$$
\operatorname{RMSE}_{\text {test }}:=\left(\frac{1}{\# I_{\text {test }}} \sum_{i \in I_{\text {test }}}\left(\hat{f}\left(X_{i}\right)-f\left(X_{i}\right)\right)^{2}\right)^{\frac{1}{2}}
$$




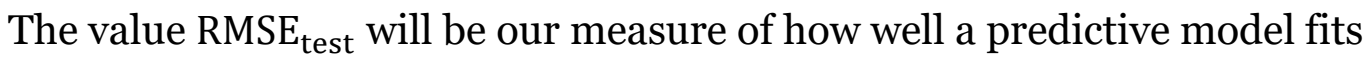
the data. The training data should not be used in this measurement to avoid overfitting.

It is common to start with linear regressors for these predictive models. Linear models are fast to train and have the added benefit of being easy to interpret. The downside to linear models is that they may not fit the data well in the sense of Equation (23). Indeed, using the linear model implementations in Alan Turing Institute (2020), we were only able to obtain $\mathrm{RMSE}_{\text {test }}=0.12$ which, put in the context of the after emplacement distribution in Section 6.4.1, appears to fit quite poorly.

We instead opt to use the flexibility of a nonlinear model to find a model


comes at the cost of interpretation which we address in Sections 6.4.5 and 6.4.6. The nonlinear model used here is a decision tree method called XGBoost (Chen and Guestrin 2016) implemented in (DDMLC 202). XGBoost is one of several popular decision tree methods. The core design principle of XGBoost is gradient boosting (Freund and Schapire 1997). The results from many decisions trees are combined to build the model from the training data by solving an optimization problem using gradient descent. Many hyperparameters (parameters that are not optimized through training) govern the performance of XGBoost (XGBoost 2020).

After sufficiently tuning the XGBoost hyperparameters we obtain RMSE $E_{\text {test }}$ results less than half the size of the linear models. Residual plots for the XGBoost model, using all input environmental variables, are shown in Figure 33 with $\mathrm{RMSE}_{\text {test }}=0.0579$. Similarly, using the subset of applicable variables (defined in Section 6.2.1), we obtain $\mathrm{RMSE}_{\text {test }}=0.0576$ and plot the residuals in Figure 34. 
Figure 33. Residual plots for XGBoost model using all environmental input variables. residual distribution : $\mathrm{RMSE}=0.0579$
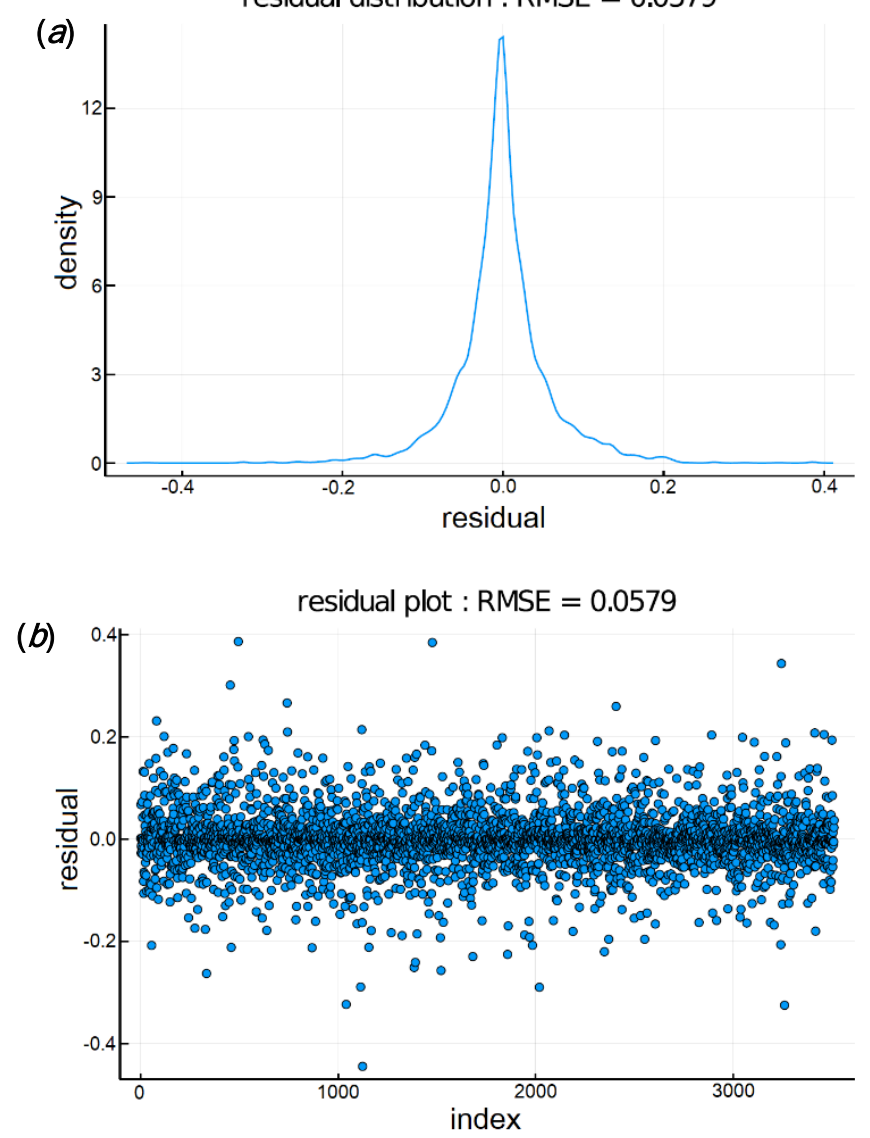

Figure 34. Residual plots for an XGBoost model using the subset of applicable environmental input variables defined in Section 6.2.1.

residual distribution : $\mathrm{RMSE}=0.0576377043$

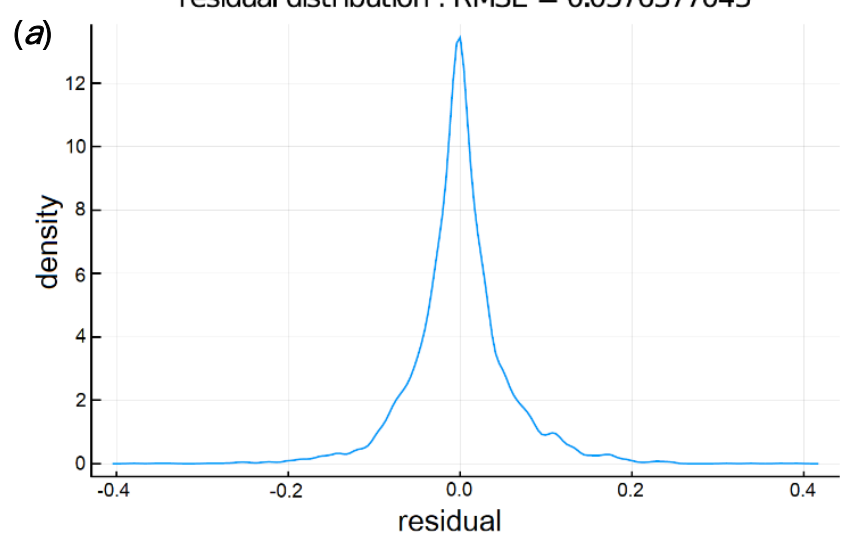




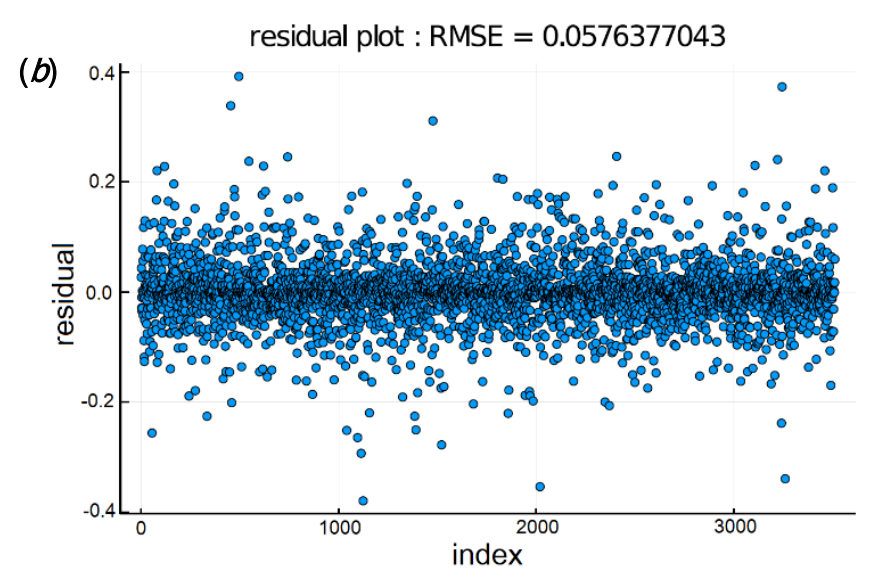

In this section, we found a sufficient nonlinear approximation for the mapping between environmental variables and the object variables using the XGBoost algorithm. This model addressed the first point of Objective 6.3 , approximating the function $f$. Next, we address the second point of Objective 6.3, namely interpreting the effects of the input environmental variables on this approximation.

\subsubsection{Nonlinear interpretation with Shapley values}

Having developed a sufficiently accurate function that predicts AUC target variables from environmental inputs in the previous section, we now aim to interpret these predictions to approach the remainder of Objective 6.3. We restrict the analysis in this section to the set of applicable environmental variables defined in Section 6.2.1.

Webegin with an approach from (Štrumbelj and Kononenko 2014) based on the approximate calculation of Shapley values implemented in (Redell 2020). Shapley values provide a measure of feature importance in a local sense by additively decomposing an observed response into a sum of effects from each input variable with respect to a baseline effect. In our case we can use Shapley values to decompose the observed AUC of an individual IR image into a baseline AUC (the average AUC over all observations) and a sum of AUC contributions (with respect to the baseline) for each environmental input. Equation (23) summarizes this decomposition in general:

\footnotetext{
observed AUC $=$ baseline effect + $\sum_{\text {features }}$ (Shapley effect for a given feature).
} 
Figure $35 a$ provides an illustration of this decomposition for a specific example. A global measure of feature importance can be obtained from these local values by computing the mean absolute Shapley value for a fixed feature. We summarize the outcomes from these computations in Figure $35 b$.

Figure 35. Decomposition of an observed AUC based on (a) Shapley effects and (b) environmental variables ordered by feature importance measured by aggregating Shapley effects in relation to the baseline effect.

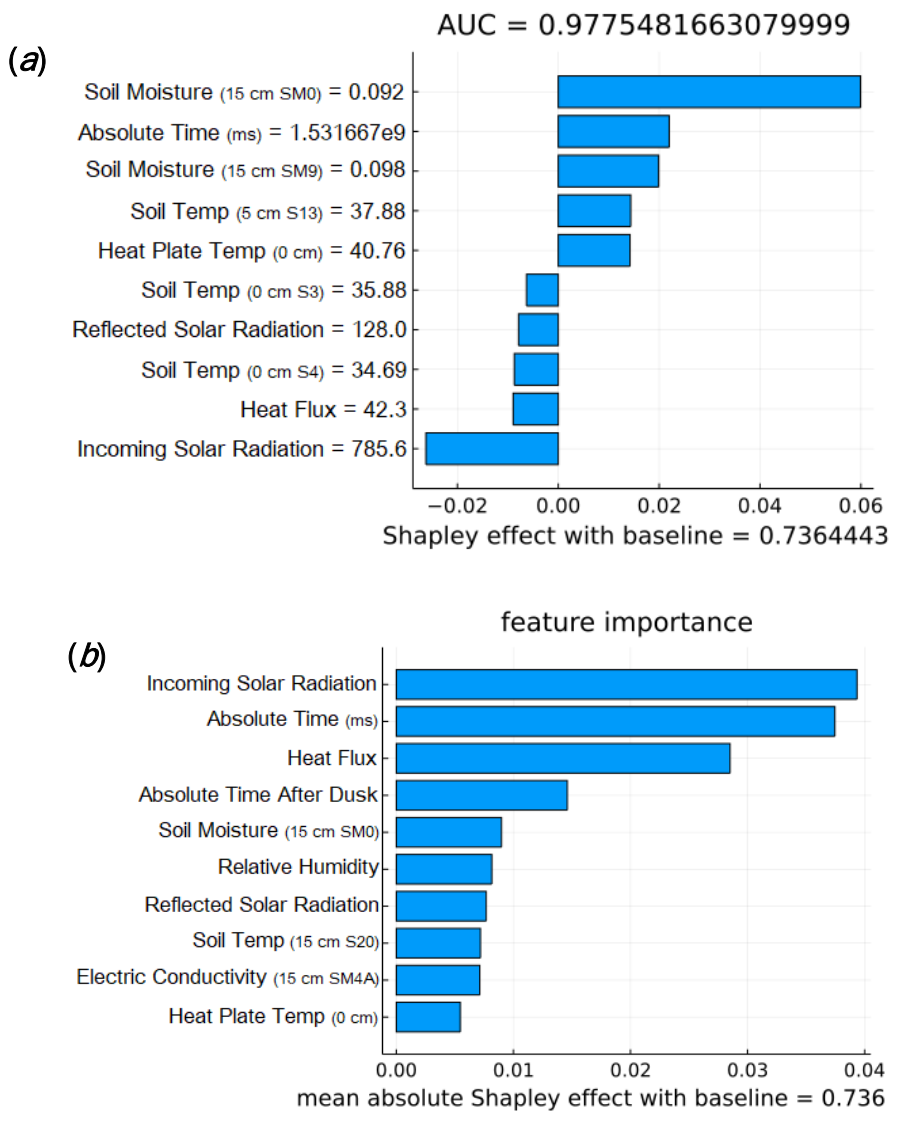

To analyze this further (toward Objective 6.3) we consider the plots of Shapley values for a fixed environmental input. We refer to these as Shapley scatter plots. These plots can then be used to determine trends in the environmental variables that influence the AUC distributions. These trends can either be analyzed visually or by some type of curve fitting procedure. In Figure 36 are Shapley scatter plots for two environmental variables of interest. On the left is the scatter plot for the heat flux variable, FLX1, and on the right is the scatter plot for the soil temperature variable at a depth of $15 \mathrm{~cm}$ below the surface in location 20 , DTLayer15_RawTmp(20). 
Figure 36. Shapley scatter plots for $(a)$ heat flux $\left(\mathrm{W} / \mathrm{m}^{2}\right)$ and $(b)$ the temperature $\left({ }^{\circ} \mathrm{C}\right)$ measured at a depth of $15 \mathrm{~cm}$ in location 20 within the plot.

(a)
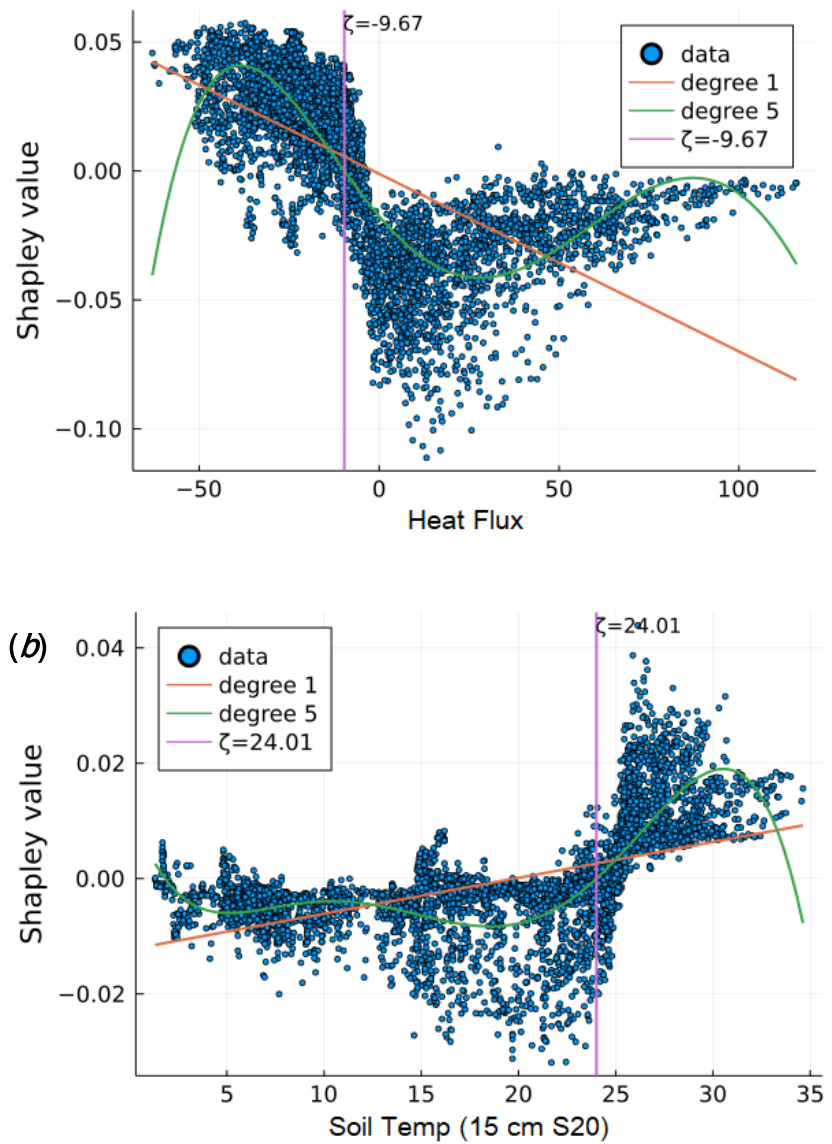

Using the roots of polynomials fit to the data, we obtain values $\zeta$ which partially address Objective 6.3. For example, in Figure 36 for the heat flux variable FLX1, we found $\zeta=-9.67\left(\mathrm{~W} / \mathrm{m}^{2}\right)$ and this value identifies an approximate cut-off value for the heat flux in which we see a change from positive to negative Shapley values. When the Shapley value is positive it indicates the environmental variable has a positive effect on the interpretation of the function that maps the environmental variables to the object variables, which is a core goal of Objective 6.3. This can further be investigated through AUC distribution tables. In Table 16, we see that the heat flux variable FLX1 tends to increase AUC when FLX1 $\leq-9.67$.

Similarly, it can be seen in Table 17 that the AUC decreases when FLX1 $\geq$ -9.67. To automate this process we fit both linear and quintic polynomials to the data. The value $\zeta$ is then obtained by taking the real root of the quintic polynomial closest to the linear root. 
Table 16. AUC distribution for the baseline of all observations made after object emplacement against observations where heat flux variable FLX1 $\leq-9.67$.

\begin{tabular}{|l|r|r|r|r|r|r|}
\hline targets & \multicolumn{1}{c|}{$\boldsymbol{\mu}_{\text {change }}$} & \multicolumn{1}{c|}{$\boldsymbol{\mu}_{\text {base }}$} & \multicolumn{1}{c|}{$\boldsymbol{\mu}_{\text {test }}$} & \multicolumn{1}{c|}{$\boldsymbol{\sigma}_{\text {base }}$} & \multicolumn{1}{c|}{$\boldsymbol{\sigma}_{\text {test }}$} & \multicolumn{1}{l|}{ Wasserstein } \\
\hline all & 0.03 & 0.766 & 0.796 & 0.189 & 0.183 & 0.0305 \\
\hline SP & 0.023 & 0.892 & 0.915 & 0.217 & 0.204 & 0.0232 \\
\hline DP & 0.039 & 0.716 & 0.754 & 0.293 & 0.29 & 0.0386 \\
\hline DM & 0.06 & 0.77 & 0.83 & 0.266 & 0.24 & 0.06 \\
\hline SM & -0.002 & 0.686 & 0.684 & 0.301 & 0.311 & 0.011 \\
\hline metal & 0.029 & 0.728 & 0.757 & 0.218 & 0.208 & 0.029 \\
\hline plastic & 0.031 & 0.804 & 0.835 & 0.205 & 0.2 & 0.0309 \\
\hline deep & 0.049 & 0.743 & 0.792 & 0.261 & 0.247 & 0.0493 \\
\hline shallow & 0.011 & 0.789 & 0.799 & 0.202 & 0.208 & 0.0132 \\
\hline
\end{tabular}

Table 17. AUC distribution for the baseline of all observations made after object emplacement against observations where heat flux variable FLX1 $\geq-9.67$.

\begin{tabular}{|l|r|r|r|r|r|r|}
\hline targets & \multicolumn{1}{c|}{$\boldsymbol{\mu}_{\text {change }}$} & \multicolumn{1}{c|}{$\boldsymbol{\mu}_{\text {base }}$} & \multicolumn{1}{c|}{$\boldsymbol{\mu}_{\text {test }}$} & \multicolumn{1}{c|}{$\boldsymbol{\sigma}_{\text {base }}$} & \multicolumn{1}{c|}{$\boldsymbol{\sigma}_{\text {test }}$} & Wasserstein \\
\hline all & -0.81 & 0.766 & 0.684 & 0.189 & 0.183 & 0.083 \\
\hline SP & -0.063 & 0.892 & 0.829 & 0.217 & 0.236 & 0.0631 \\
\hline DP & -0.105 & 0.716 & 0.611 & 0.293 & 0.273 & 0.1051 \\
\hline DM & -0.163 & 0.77 & 0.606 & 0.266 & 0.265 & 0.1634 \\
\hline SM & 0.006 & 0.686 & 0.692 & 0.301 & 0.272 & 0.0301 \\
\hline metal & -0.079 & 0.728 & 0.649 & 0.218 & 0.226 & 0.0789 \\
\hline plastic & -0.084 & 0.804 & 0.72 & 0.205 & 0.194 & 0.0841 \\
\hline deep & -0.134 & 0.743 & 0.609 & 0.261 & 0.252 & 0.1343 \\
\hline shallow & -0.029 & 0.789 & 0.76 & 0.202 & 0.183 & 0.036 \\
\hline
\end{tabular}

The Shapley values partially address Objective 6.3. In particular, the environmental variable with the largest positive Shapley value identifies which environmental variables have the largest effect on the object variable, addressing Objective 6.3.1. Further, through the polynomial fitting of the Shapley values, as shown in Figure 36, we can identify specific ranges of the environmental variables to determine how a value for that variable in the identified range will affect the prediction of the object variable, as was discussed for the heat flux variable above. Although the Shapley values begin to address Objective 6.3, there are some drawbacks. The first is that the Shapley computations rely on linearity assumptions. The second is that it is unclear (at the moment) how to deal with interaction effects between the environmental variables to determine second-order effects, which is the topic of Objective 6.3.3. For example, the Shapley analysis determined that the incoming solar radiation was the most important environmental effect in the relationship between 
environmental variables and predicting the object variables. However, it would be helpful to understand which combination of environmental variables paired with the incoming solar radiation lead to this strong effect in the approximate model. It is certainly possible to analyze interaction effects with Shapley values (Lundberg et al. 2018, 2020; Lundberg and Lee 2017), but this requires further effort to apply these techniques to our application. The next section begins to address the second shortcoming of the Shapley value analysis and helps to address Objective 6.3 more completely.

\subsubsection{Nonlinear interpretation with global sensitivity analysis}

To address the nonlinear interaction effects involved with interpreting the models defined in Section 6.4.4, we turn to sensitivity analysis. Global sensitivity analysis techniques are used to measure the contributions of input variables and their interactions with the overall uncertainty of the output. In the following analysis we use the Sobol method (Saltelli 2002, 2010; Sobol 2001) following the implementation details in Rennels (2020), SciML Ecosystem (2020), Rackauckas and Nie (2017), and Usher et al. (2016).

In what follows we only consider the subset of applicable environmental variables defined in Section 6.2.1. Let $y$ denote the AUC object variable defined in Section 6.3.1 with sampling based on all buried objects. By considering all objects together, we can treat $y$ as univariate (instead of being composed of nine variables) which simplifies the sensitivity analysis.

The Sobol method for sensitivity analysis decomposes the variance of $y$ into contributions from subsets of inputs in the following sense. Let $k$ be the number of input environmental variables $x_{1}, \ldots, x_{k}$, and let $y$ be represented as

$$
y=f_{0}+\sum_{i=1}^{k} f_{1}\left(x_{k}\right)+\sum_{i<j}^{k} f_{i, j}\left(x_{i}, x_{j}\right)+\cdots+f_{1,2, \ldots, k},
$$

where $y$ is decomposed into a background response given by $f_{0}$, and firstorder responses given by $f_{1}$, and higher-order interactions between the environmental variables. With the representation of $y$ given in Equation (24), we can write the variance of the output variable as follows:

$$
\operatorname{var}(\mathrm{y})=\sum_{i=1}^{k} v_{i}+\sum_{i<j}^{k} v_{i, j}+\cdots+v_{1,2, \ldots, k}
$$


The first and second order Sobol indices are defined by:

$$
S_{i}:=\frac{v_{i}}{\operatorname{var}(\mathrm{y})} \text {, and } S_{i, j}:=\frac{v_{i, j}}{\operatorname{var}(\mathrm{y})},
$$

respectively. The Sobol total sensitivity (including all higher order nonlinear interactions) for a given environmental input $x_{i}$ is denoted by $\mathrm{ST}_{i}$.

Figure 37 lists the environmental inputs with the highest total sensitivity (left) and highest first order sensitivity (right). A large discrepancy between total sensitivity and first order sensitivity indicates the presence of interaction effects between the environmental variables (e.g. incoming solar radiation in Figure 37). Notably, both the total sensitivity and the first order sensitivity both identify the incoming solar radiation as the environmental variable with the largest effect on the object variable $y$ and this finding agrees with the Shapley analysis in the previous section. Figure 38 lists the environmental variables whose second order Sobol indices are highest when paired with incoming solar radiation. The second order Sobol indices for a given input variable can help determine what the most prominent second order interactions are for the fixed input variable. In the example for incoming solar radiation shown in Figure 38, the most important second order interactions are given by the heat flux and absolute time in the day.

Figure 37 . Sobol (a) total sensitivity and $(b)$ first order sensitivity sorted by variance ratio.

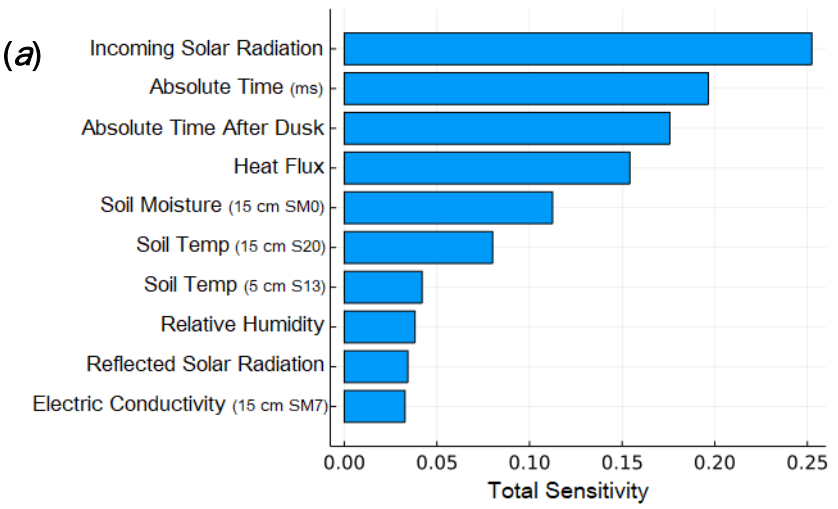




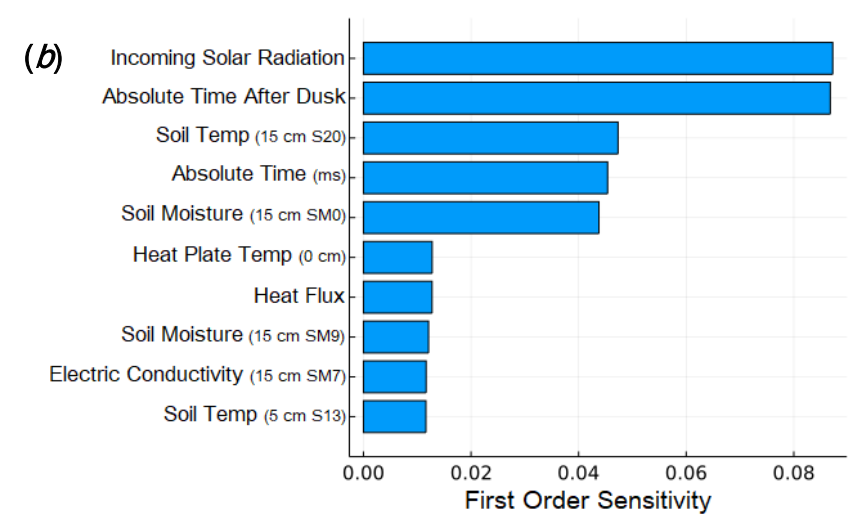

Figure 38. Highest second order Sobol indices associated with the average incoming solar radiation.

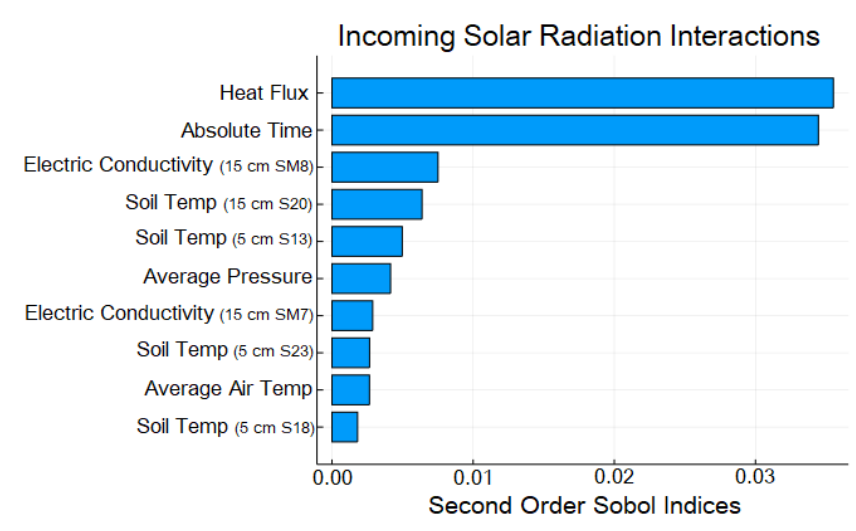

\subsection{Future directions}

We conclude by outlining some possible future directions for this work.

\subsubsection{Target variables}

A great deal of effort has been put forth to study contrast metrics and detection algorithms in a wide variety of contexts (Schachter 2012, 2018, 2020; Ahuja and Schachter 1981), and the task to determine the appropriate response variable appears to be a subtle topic. Since we are not necessarily attempting to construct a high-performance detection algorithm with this work, it is possible that the object variable we choose will not affect the results of the environmental studies. It is also possible, however, that we miss key points of the analysis based on a poor choice of target variable. IR image feature engineering for a specific application is likely to be necessary to obtain the most accurate and relevant results in a particular situation. Since the methods outlined in this work are general, the framework should require minimal changes to adapt the analysis to a new response. 


\subsubsection{Alternate approaches}

The data analysis approaches applied to date relied on asophisticated, nonlinear regressor to identify the variable impacting object detection using thermal IR. Further investigation of applicable dimension reduction techniques and clustering methods are being evaluated. Another approach is to group environmental variables in a reasonable way to reduce the dimension of the problem. This could range from a simple solution, like averaging groups of sensors, to a more complicated mixed model approach.

Lastly, an analysis of detection algorithms that include environmental data could also be explored. Note, that this is subtly different than the framework used in this work. Here we do not provide environmental information to the detection algorithms. AUC is computed without any knowledge of the environmental data, and the environmental effects are analyzed afterwards. Measuring how detection performance changes based on environmental data provided to the detection algorithm is perhaps another approach worth pursuing. Causal models such as those described in Redell (2019) and Tavares et al. (2019) could be applied in our context.

\subsubsection{Time series analysis}

Perhaps the most promising approach is incorporating the sequential aspect of the data. Several simple attempts at addressing the temporal analysis were made and include:

1. Input variables added to the data to encode important features related to time. For example, considering the environmental conditions leading up to a particular observation/IR image.

2. Clock diagrams based on grouping data by time of day.

3. The global search regression technique from the econometrics literature (Ungaro et al. 2020) was analyzed. Unfortunately, the linear model fit was poor (the best RMSE obtained was approximately 0.13).

However, a thorough treatment of the data beyond the snapshot assumption is necessary. Time series classification and clustering should be considered (Kidger et al. 2020; Loning et al. 2019; Teichbraeber et al. 2019; Christ et al. 2018; Giannakis and Majda 2012). 


\subsubsection{Extrapolation and validation}

Environmental results can be validated through physical modeling, additional field experiments, and from a demonstrated improvement in detection algorithms. Testing environmental results and having those tests inform future analysis is crucial to make real progress in this area. 


\section{Findings and Conclusion}

This work is a comprehensive follow on analysis of the data collected and discussed in the Phase 2 report (Clausen et al. 2021). Here we have expanded on the analysis of the Phase 2 data to include a multipronged analytical approach as follows: (1) multivariate analyses for detecting objects in soil, (2) a moving box analysis of object visibility with alternative methods for converting FLIR radiance values to thermal temperature values, (3) a calibrated thermal model of soil temperature using thermal IR imagery, and (4) a simple classifier method for automating buried object detection. In addition, we have expanded our field testing to include a 2-week test at the YPG Countermine test site and compared object visibility with the CRREL test plot thermal imagery-to be reported in a subsequent report.

Environmental phenomenological effects are often represented in weather forecasts in a relatively coarse, hourly resolution, which introduces concerns such as exclusion or misrepresentation of ephemera or lags in timing when using this data as an input for the TAK software system. It is unclear if data from field programs examining the detection of buried targets will support the hypothesis that enhanced surface detection is well correlated with rapid changes in near surface temperatures. Additionally, the direct application of observed temperature data with weather model data may not be the best approach because metadata associated with the observations is not included. For example, the local observation may differ from the data incorporated in the model in terms of altitude, land usage (e.g. vegetation type and coverage), or soil texture. As a result, there is a need to explore mathematical methods such as Bayesian statistics to incorporate observations into models.

Investigation of buried object detection using multilevel analyses revealed that IR temperatures of the thermal IR imagery along with soil temperature at $5 \mathrm{~cm}$ provide detection of the contrast of soil over buried objects compared with the surrounding soil at varying magnitude, with SP tending to be the most easily detectable and SM second. The reason for this variability is that metals are more conductive of heat energy, and thus the plastic object tends to heat up more slowly than the surrounding soil and to retain heat for a longer period as the surrounding soil cools. DP and DM objects are much less detectable using IR temperature and soil temperature at $5 \mathrm{~cm}$. Of the environmental phenomenological variables 
affecting contrast, soil temperature and soil moisture were the greatest contributors. Other variables such as air temperature, relative humidity, incoming short-wave radiation, and wind speed did not appear to have a direct effect on detecting buried objects. ROC analysis supports these findings.

Because it was not known how the thermal temperatures were derived from FLIR radiance values using the FLIR Systems algorithm, we investigated two methods for converting FLIR radiance values to thermal temperature values. These methods correct for air temperatures, relative humidity, and emissivity. Using the weather data measured at the CRREL test site rather than the default weather parameters used in the FLIR algorithm, both of the non-FLIR methods yield the same results.

Employing temperature values derived from these methods, we performed a moving box analysis of thermal IR imagery from July 2018-December 2018. PD using this analysis was higher as box overlap decreased. Additionally, the SP object was most easily detectable, with the FAR for the SM, DP, and DM objects being relatively higher and PD lower. This effort will be expanded over FY21 to look at how dependencies change seasonally, with a focus on frozen soil and snow thickness/density.

With our third analytical approach, we developed and calibrated a thermal model to improve buried object detection by using soil temperature measurements at $5 \mathrm{~cm}$ and thermal IR imagery temperatures as boundary conditions. In general, the soil temperatures simulated by the model exhibited a better fit when the measured temperatures at $5 \mathrm{~cm}$ were used as opposed to the thermal IR imagery temperatures. When the methods noted above for deriving thermal temperatures from FLIR radiances were used in place of the FLIR algorithm, some time periods were found to fit better in the model using the non-FLIR methods, and others fit better using the FLIR method. We concluded that this discrepancy indicates a need for more careful calibration of the thermal imagery conversion parameters in order to yield a closer match to surface temperatures. To avoid this problem and improve efficiency, it would be ideal to skip the conversion process altogether and use the raw FLIR radiances for buried object analysis as actual temperatures are not needed for object detection.

In the Phase 2 report (Clausen et al. 2021), we introduced a simplistic differencing scheme using FLIR radiance values for automatically generating a VI of buried objects. Here we have developed an improved 
classifier, which generates AUC values for nine discrete sections of the test plot using raw FLIR radiance values of both pre-object emplacement and post-object emplacement thermal IR images. The AUC value generated can be used as a metric to determine visibility for each buried object. The relative effect of the different variables on object visibility was determined by using a nonlinear interpretation of this method with Shapely values on environmental variables such as solar loading, soil moisture, air temperature, and soil conductivity having the greatest effect, in that order. We are currently developing more sophisticated methods for automating object visibility through machine learning (ML)/artificial intelligence (AI) data analysis of the CRREL test plot and YPG test data. The new classifier will be trained on our existing thermal IR imagery database for 2018 and 2020 and imagery collected during the YPG field test to make a decision automatically and more accurately on the visibility of buried objects.

In addition to a secondary testing site at YPG, we are also in the process of testing a number of variables in isolation at the CRREL field test site as well with a physics-based model (Trautz et al. 2021) to help validate our findings. These variables include wind speed, solar radiation, camera angle, soil moisture, surface topology, and soil density. Additional snow density, and surface object tests were conducted over winter 2020, and a final test of surface object visibility using the same hockey pucks that were used for the YPG test is currently in progress.

This research effort will continue through FY21 as we expand on and improve our analyses and models. We plan to (1) explore the development of ML/AI approaches for analyzing data from the CRREL test plot in an effort to identify the variables that impact ATR performance; (2) complete the remaining field manipulation tests and perform a full analysis of the resulting test data; (3) develop a physics-based model for the YPG test plot and calibrate, validate, and compare with field observations; (4) summarize thermal imagery and environmental sensor field data collected over 2018-2021, (5) explore seasonal impacts on soil and object thermal response as it relates to object detectability, (6) develop an ML-based preprocessor accounting for environmental phenomenology effects that can be linked to standard Army ATRs for improving object detection performance, and (7) develop approaches for efficiently generating AI/ML synthetic imagery in support of ML algorithms for object detection. The findings from these tasks will be presented in a follow-on report. 


\section{References}

Abbott, R., N. M. Robertson, J. M. del Rincon, and B. Connor. 2020. "Unsupervised Object Detection via LWIR/RGB Translation." 2020 IEEE/CVF Conference on Computer Vision and Pattern Recognition Workshops (CVPRW): 407-15. Seattle, WA. https://doi.org/10.1109/CVPRW50498.2020.00053.

Affleck, R. T., K. Gardner, S. Aytur, C. Carlson, C. Grimm, and E. Deeb. 2019. "Sustainable Infrastructure in Conflict Zones: Police Facilities' Impact on Perception of Safety in Afghan Communities." Sustainability 11 (7): 2113. https://doi.org/10.3390/su11072113.

Ahuja, N., and B. J. Schachter. 1981. "Image Models." ACM Computing Surveys (CSUR) 13 (4): 373-97.

Alan Turing Institute. 2020. Mljlinearmodels.jl. Accessed December 2020. https://github.com/alan-turing-institute/MLLinearModels.jl.

Baker, J. M., D. C. Reicosky, and D. G. Baker. 1989. "Estimating the Time Dependence of Air Temperature Using Daily Maxima and Minima." Journal of Atmospheric and Oceanic Technology 5: 736-42.

Ballard, J. R., S. E. Howington, and S. C. Wilhelms. 2013. "Laboratory-based Rainfall Effects on LWIR Soil Reflectance.” IEEE Geoscience and Remote Sensing Letters 10 (3): 627-30. https://doi.org/:10.1109/LGRS.2012.2216250.

Baur, J., L. Zheng, W. Frazer, A. Nikulin, T. S. de Smet. 2018. "Improved Detection of Plastic Landmines with Aerial Polarized Thermal Imaging." AGUFM 2018: NS33B-0792.

Blaom, A. D., F. Kiraly, T. Lienart, Y. Simillides, D. Arenas, and S. J. Vollmer. 2020. MLJ: A Julia Package for Composable Machine Learning. arXiv Preprint. Ithaca, NY: Cornell University. https://arxiv.org/abs/2007.12285v2.

Chang, C., D. A. Laird, M. J. Mausbach, and C. R. Hurburgh Jr. 2001. "Near-Infrared Reflectance Spectroscopy-Principal Components Regression Analyses of Soil Properties.” Soil Science Society of America Journal 65: 4870-4890.

Chen, T., and C. Guestrin. 2016. "Xgboost: A Scalable Tree Boosting System.” Proceedings of the 22nd ACM SigKDD International Conference on Knowledge Discovery and Data Mining: 785-94.

Christ, M., N. Braun, J. Neuffer, and A. W. Kempa-Liehr. 2018. "Time Series Feature Extraction on Basis of Scalable Hypothesis Tests (Tsfresh-A Python Package)." Neurocomputing 307: 72-77.

Chung, S.-O., and R. Horton. 1987. "Soil Heat and Water Flow with a Partial Surface Mulch." Water Resources and Research 23 (12): 2175-86. https://doi.org/10.1029.WR023i012p02175. 
Clausen, J. L., S. Frankenstein, J. R. Dorvee, A. Workman, B. F. Moriss, K. J. Claffey, T. M. Sobecki, et al. 2021. Spatial/Temporal Variance of Soil and Meteorological Properties Affecting Sensor Performance-Phase 2. ERDC/CRREL TR-21-10. Hanover, NH: U.S. Army Corps of Engineers, Engineer Research and Development Center, Cold Regions Research and Engineering Laboratory.

Clausen, J. L., J. R. Dorvee, A. Wagner, S. Frankenstein, B. F. Moriss, K. J. Claffey, T. M. Sobecki, et al. 2020. Spatial/Temporal Variance in the Thermal Response of Buried Objects. ERDC/CRREL TR-20-10. Hanover, NH: U.S. Army Corps of Engineers, Engineer Research and Development Center, Cold Regions Research and Engineering Laboratory.

Deans, J., G. Schmithals, and L. J. Carter. 2001. "An Analysis of a Thermal Imaging Method for Landmine Detection Using Microwave Heating." Journal of Applied Geophysics 47: 123-33. https://doi.org/10.1016/S0926-9851(01)00052-0.

de Wit, C. T. 1978. Simulation of Assimilation, Respiration, and Transpiration of Crops. Wageningen, NE: Pudoc, 148.

DDMLC. 2020. "Distributed (Deep) Machine Learning Community. 2014-2020. Xgboost.jl." Accessed December 2020. https://github.com/dmlc/XGBoost.jl.

Dogaru, T., and C. Le. 2016. "Polarization Differences in Airborne Ground Penetrating Radar Performance for Landmine Detection.” Proc. SPIE 9829, Radar Sensor Technology XX, 9829oB. https://doi.org/10.1117/12.2227877.

Ek, M. B., K. E. Mitchell, Y. Lin, E. Rogers, P. Grunmann, V. Koren, G. Gayno, and J. D. Tarpley. 2003. "Implementation of Noah Land Surface Model Advances in the National Centers for Environmental Prediction Operational Mesoscale Eta Model." Journal of Geophysical Research 108 (D22). https://doi.org/10.1029/2002JD003296.

Ene, M., E. A. Leighton, G. L. Blue, and B. A. Bell. 2015. "Multilevel Models for Categorical Data Using SAS ${ }^{\circledR}$ PROC GLIMMIX: The Basics.” In SAS Global Forum 2015 Proceedings, 25-29 April, Dallas, TX, Paper 3430-2015.

Ewing, K. J., K. J. Major, J. Sanghera, R.V. Kala, S.E. Howington, and J. Ballard. 2020. "Behavior of the Reststrahlen Band in the 17-25 $\mu \mathrm{m}$ Spectral Region in the Diffuse Reflection Spectra of Sand and Silt Mixtures." Applied Spectroscopy 74 (3): 334-39. https://doi.org/10.1177/0003702819884323.

FLIR Systems. 2015. The Ultimate Infrared Handbook for R\&D Professionals.

Frankenstein, S., and G. G. Koenig. 2004. Fast All-season Soil STrength (FASST). ERDC/CRREL SR-04-1. Hanover, NH: U.S. Army Corps of Engineers, Engineer Research and Development Center, Cold Regions Research and Engineering Laboratory.

Freund, Y., and R. E. Schapire. 1997. "A Decision-Theoretic Generalization of On-line Learning and an Application to Boosting." Journal of Computer and System Sciences 55 (1): 119-39.

Frost, M. 2006. "Land Mine.” Accessed December 2020. http://www.martinfrost.ws/htmlfiles/may2006/landmine.html. 
Garson, G. D. 2012. Testing Statistical Assumptions. Asheboro, NC: Statistical Associates Publishing.

Giannakis, D., and A. J. Majda. 2012. "Nonlinear Laplacian Spectral Analysis for Time Series with Intermittency and Low-Frequency Variability." Proceedings of the National Academy of Sciences 109 (7): 2222-27.

Gorman, M. 1957. "The Evidence from Infrared Spectroscopy for Hydrogen Bonding." Journal of Chemical Education 34 (6): 304-6.

Gurton, K. P., and M. Felton. 2012. "Remote Detection of Buried Land-Mines and IEDs Using LWIR Polarimetric Imaging.” Optics Express 20 (20): 22344-59.

Harvey, P. 2013. ExifTool Forum, Topic: Enhancement: Extract Binary Data from FLIR Radiometric jpg by tomas123. https://exiftool.org.forum/index.php?topic=4898.60.

Heck, R. H., and S.L. Thomas. 2000. An Introduction to Multilevel Modeling Techniques. Mahwah, NJ: Lawrence Erlbaum Associates.

Hedeker, D. 2005. "Generalized Linear Mixed Models. Encyclopedia of Statistics in Behavioral Science. https://doi.org/10.1002/0470013192.bsa251.

Hendrickx, J. M. H., N. Alkov, S. Hong, R. I. Van Dam, J. Kleissl, H. Shannon, J. Meason, B. Borchers, and R. S. Harmon. 2006. "New Mexico Tech Landmine, UXO, IED Detection Sensor Test Facility: Measurements in Real Field Soils.” Proc. SPIE 6217, Detection and Remediation Technologies for Mines and Minelike Targets XI, 62170V. https://doi.org/10.1117/12.665644.

Hong, S., T. W. Miller, B. Borchers, J. M. H. Hendrickx, H. K. Lensen, P.B.W. Schwering, and S.P. van den Broek. 2002. "Land Mine Detection in Bare Soils Using Thermal Infrared Sensors." Proc. SPIE 4742, Detection and Remediation Technologies for Mines and Minelike Targets VII (13 August 2002). https://doi.org/10.1117/12.479124.

Hosmer, D., and S. Lemeshow. 2000. Applied Logistic Regression. 2nd ed. Hoboken, NJ: John Wiley and Sons.

Howington, S. 2020. Personal communication with J. Clausen, February 2020.

Howington, S. E., J. R. Ballard Jr., N.V. Kala, A.C. Trautz, M.D. Bray, M.W. Farthing, and A.M. Hines. 2019. "Exploitable Synthetic Sensor Imagery from High-Fidelity, Physics-Based Target and Background Modeling." Proceedings of SPIE 11158, Target and Background Signatures V: 1115809. https://doi.org/10.1117/12.2532923.

Howington, S. E., J. F. Peters, J. R. Ballard, O. J. Eslinger, J. R. Fairley, R. V. Kala, R. A. Goodson, et al. 2012. "Using Computer Simulation to Explore the Importance of Hydrogeology in Remote Sensing for Explosive Threat Detection." Geological Society London Special Publications 362: 287-300. https://doi.org/10.1144/SP362.16.

Hox, J. J. 2002. Multilevel Analysis: Techniques and Applications. Mahwah, NJ: Lawrence Erlbaum Associates. 
Hudson, R., and J. Hudson. 1975. "The Military Applications of Remote Sensing by Infrared." Proceedings of IEEE Geoscience and Remote Sensing Letters 63: 10428.

Jarrod, P., M. Brown, C. Wagner, R. G. Roberts, and D.B. Card. 2019. "Experiments in Detecting Obscured Objects Using Longwave Infrared Polarimetric Passive Imaging." Proceedings of SPIE 11001, Infrared Imaging Systems: Design, Analysis, Modeling, and Testing XXX: 1100107. https://doi.org/10.1117/12.2518547.

Kabessa, Y., O. Eyal, B. Ofer, V. Korouma, S. Yagur-Kroll, S. Belkin, and A. Agranat. 2016. "Standoff Detection of Explosives and Buried Landmines Using Fluorescent Bacterial Sensor Cells." Biosensors and Bioelectronics 79 (15): 784-88. https://doi.org/10.1016/i.bios.2016.01.011.

Kelly, C. D., B. R. Phelan, T. V. Dogaru, K. D. Sherbondy, and R. M. Narayanan. 2019. "Investigation of Airborne Synthetic Aperture Radar Parameters for Buried Target Detection." Proceedings of SPIE 11003, Radar Sensor Technology XXIII: 1100306. https://doi.org/10.1117/12.2519674.

Kennedy, S. 2020. "Python Package 2009-2020: Astral.” Accessed December 2020. https://astral.readthedocs.io/en/latest/.

Khanafer K., and K. Vafai. 2002. "Thermal Analysis of Buried Land Mines over a Diurnal Cycle." IEEE Transactions on Geoscience and Remote Sensing 40 (2): 461-73.

Kidger, P., J. Morrill, J. Foster, and T. Lyons. 2020. "Neural Controlled Differential Equations for Irregular Time Series." arXiv Preprint. Ithaca, NY: Cornell University. https://arxiv.org/abs/2005.08926v2.

Klein, K. J., and S. W. Kozlowski. 2000. Multilevel Theory, Research, and Methods in Organizations: Foundations, Extensions and New Directions. San Francisco: Jossey-Bass.

Koenig. G., Y. Koh., S. Howington, and C. Scott. 2008. "Phenomenology of Thermal Signatures of Disturbed and Undisturbed Soils." Proceedings of SPIE. 6953, Detection and Sensing of Mines, Explosive Objects, and Obscured Targets XIII. https://doi.org/ 10.1117/12.777790.

Koh, G., and L. D. Wakeley. 2009. "Attenuation of Electromagnetic Energy in Soil from Yuma Proving Ground.” ERDC TR-09-9. Hanover, NH: U.S. Army Corps of Engineers, Engineer Research and Development Center, Cold Regions Research and Engineering Laboratory.

Lamorski, K., P. Prezgowski, W. Swiderski, D. Szabra, R. T. Walczak, and B. Usowic. 2002. "Thermal Signatures of Land Mines Buried in Mineral and Organic SoilsModelling and Experiments." Infrared Physics \& Technology 43: 303-9.

Li, H., Y. Wang, H. Chang, W. Xiao, Z. Yu, and S. Guo. 2020. "Acoustic Impedance and Its Application in Seismo-Acoustic Landmines Detection Models.” Journal of Coastal Research 99 (sp1): 92-98.

Li, K. 1991. “Sliced Inverse Regression for Dimension Reduction.” Journal of the American Statistical Association 86 (414): 316-27. 
Löning, M., A. Bagnall, S. Ganesh, V. Kazakov, J. Lines, and F. J. Király. 2019. "sktime: A Unified Interface for Machine Learning with Time Series,” Workshop on Systems for ML at NeurIPS.

Lundberg, S. M., G. Erion, H. Chen, A. DeGrave, J. M. Prutkin, B. Nair, R. Katz, et al. 2020. "From Local Explanations to Global Understanding with Explainable AI for Trees." Nature Machine Intelligence 2 (1): 2522-5839.

Lundberg, S. M., and S. Lee. 2017. "A Unified Approach to Interpreting Model Predictions." In Advances in Neural Information Processing Systems 30, edited by I. Guyon, U. V. Luxburg, S. Bengio, H. Wallach, R. Fergus, S. Vishwanathan, and R. Garnett, 4765-74. Red Hook, NY: Curran Associates, Inc.

Lundberg, S. M., B. Nair, M. S. Vavilala, M. Horibe, M. J. Eisses, T. Adams, D. E. Liston, et al. 2018. "Explainable Machine-Learning Predictions for the Prevention of Hypoxaemia during Surgery." Nature Biomedical Engineering 2 (10): 749-60.

Malof, J. M., and L. M. Collins. 2016. "Using Queuing Models to Aid Design and Guide Research Effort for Multimodality Buried Target Detection Systems.” Proceedings of SPIE 9823, Detection and Sensing of Mines, Explosive Objects, and Obscured Targets XXI: 98231K. https://doi.org/10.1117/12.2224131.

Mandrekar, J. N. 2010. "Receiver Operating Characteristic Curve in Diagnostic Test Assessment.” Journal of Thoracic Oncology 5 (9): 1315-16.

McCulloch, C. E., and S. R. Searle. 2001. Generalized, Linear, and Mixed Models. New York: Wiley.

McInnis, K. J. 2018. “The 2018 National Defense Strategy. CRS Reports. (Library of Congress. Congressional Research Service). https://purl.fdlp.gov/GPO/gpo142891.

Menard, S. 2002. Applied Logistic Regression Analysis. 2nd ed. (Vol. 106). Thousand Oaks, CA: SAGE Publishing, 111.

Miller, T. W., J. M. H. Hendrickx, and B. Borchers. 2004. "Radar Detection of Buried Landmines in Field Soils." Vadose Zone Journal 3 (4): 1116-27.

Miller, T., B. Borchers, J. M. H. Hendrickx, S. Hong, H. A. Lensen, P. B. W. Schwering, and J. Rhebergen. 2002. "Effect of Soil Moisture on Landmine Detection Using Ground Penetration Radar." Proceedings of SPIE 4742, Detection and Remediation Technologies for Mines and Minelike Targets VII. https://doi.org/10.1117/12.479099.

Musty, M., V. Truong, J. L. Clausen, S. Frankenstein, C. Williams, A. Trautz, S. Howington, and R. Kala. 2021 (In Press). Thermal Infra-red Comparison Study of Buried Objects between Humid and Desert Test Beds. ERDC-TR-2021-xx. Hanover, NH: U.S. Army Corps of Engineers, Engineer Research and Development Center, Cold Regions Research and Engineering Laboratory.

Nelder, J. A., and R. W. M. Wedderburn. 1972. “Generalized Linear Models.” Journal of the Royal Statistical Society Series A 135: 370-84.

Pan, H. L., and L. Mahrt. 1987. "Interaction between Soil Hydrology and Boundary-Layer Development.” Boundary-Layer Meteorology 38 (1-2): 185-202. 
Peters, J. F., S. E. Howington, J. Ballard Jr., and L. N. Lynch. 2007. "Signature Evaluation for Thermal Infrared Countermine and IED Detection Systems.” In DoD High Performance Computing Modernization Program Users Group Conference. New York: IEEE, 238-46.

Pinar, A. J., T. C. Havens, and A. Webb. 2017. "Multisensor Fusion of FLGPR and Thermal and Visible-Spectrum Cameras for Standoff Detection of Buried Objects." Proceedings of SPIE 10182, Detection and Sensing of Mines, Explosive Objects, and Obscured Targets XXII: 101821A. https://doi.org/10.1117/12.2262674.

Price, J. C. 1985. "On the Analysis of Thermal Infrared Imagery: The Limited Utility of Apparent Thermal Inertia. Remote Sensing of Environment 18: 59-73.

PyDataBlog. 2020. Parallelkmeans.jl. Accessed December 2020. https://github.com/PyDataBlog/ParallelKMeans.jl.

R Core Team. 2020. R: A Language and Environment for Statistical Computing. Vienna, Austria: R Foundation for Statistical Computing.

Rackauckas, C., and Q. Nie. 2017. "Differential equations. jl-A Performant and FeatureRich Ecosystem for Solving Differential Equations in Julia.” Journal of Open Research Software 5 (1): 15. http://doi.org/10.5334/jors.151.

Raqueno, R., R. Kremens, C. Salvaggio, M. Montanaro, and R. Gelein. 2008. "Humidity Effects on Thermal Atmospheric Transmissions: Study of Potential Effects of Small Hygroscopic Aerosol Particles in the Longwave Infrared Region." IGARSS 2008-2008 IEEE International Geoscience and Remote Sensing Symposium, Boston, MA, IV-1391-IV-1394, https://doi.org/ 10.1109/IGARSS.2008.4779992.

Raudenbush, S. W., and A. S. Bryk. 2002. Hierarchical Linear Models: Applications and Data Analysis Methods. 2nd ed. Thousand Oaks, CA: SAGE Publishing.

Redell, N. 2019. Shapley Decomposition of R-Squared in Machine Learning Models. arXiv preprint. Ithaca, NY: Cornell University. https://arxiv.org/abs/1908.09718v1.

Redell, N. 2020. “ShapML.” Accessed December 2020. https://github.com/nredell/ShapML.jl.

Rennels, L. 2020. “Global Sensitivity Analysis.” Accessed December 2020. https://github.com/Irennels/GlobalSensitivityAnalysis.jl.

Saltelli, A. 2002. "Making Best Use of Model Evaluations to Compute Sensitivity Indices." Computer Physics Communications 145 (2): 280-97.

Saltelli, A., P. Annoni, I. Azzini, F. Campolongo, M. Ratto, and S. Tarantol. 2010. "Variance Based Sensitivity Analysis of Model Output: Design and Estimator for the Total Sensitivity Index." Computer Physics Communications 181 (2): 259-70.

Sagnard, F. 2017. "Numerical Study of the Polarization Effect of GPR Systems on the Detection of Buried Objects." 19th EGU General Assembly, EGU2017 Proceedings. Vienna, Austria. EGUGA1919070S.

SAS 2020. "LOGISTIC Procedure Documentation, SAS 9.4." Accessed May 2021. https://documentation.sas.com/doc/en/pgmsascdc/9.4_3.4/statug/statug_logistic_details4 7.htm. 
Schachter, B. J. 2012. “Target-Detection Strategies.” Optical Engineering 52 (4): 041102. https://doi.org/10.1117/1.0E.52.4.041102.

Schachter, B. J. 2018. Automatic Target Recognition. 3rd edition. Bellingham, WA: Society of Photo-Optical Instrumentation Engineers.

Schachter, B. J. 2020. Automatic Target Recognition. 4th edition. Bellingham, WA: Society of Photo-Optical Instrumentation Engineers.

SciML Ecosystem. 2020. “DiffEqSensitivity.” Accessed December 2020. https://github.com/SciML/DiffEqSensitivity.jl.

Scott, D. W. 2015. Multivariate Density Estimation: Theory, Practice, and Visualization. Hoboken, NJ: John Wiley \& Sons.

Shaw, D., K. Stone, K. C. Ho, J. M. Keller, R. H. Luke, and B. P. Burns. 2016. “Sequential Feature Selection for Detecting Buried Objects Using Forward Looking Ground Penetrating Radar." Proceedings of SPIE 9823, Detection and Sensing of Mines, Explosive Objects, and Obscured Targets XXI, 98231L. https://doi.org/10.1117/12.2224272.

Silverman, B. W. 1982. "Algorithm as 176: Kernel Density Estimation Using the Fast Fourier Transform.” Journal of the Royal Statistical Society. Series C. Applied Statistics 31 (1): 93-99.

Smits, K. M., T. Sakaki, S. E. Howington, J. P. Peters, and T. H. Illangasekare. 2013. "Temperature Dependence of Thermal Properties of Sands across a Wide Range of Temperatures $\left(30-70^{\circ} \mathrm{C}\right)$." Vadose Zone Journal 12 (1): vzj2012.0033. https://doi.org/10.2136/vzj2012.0033.

Smits, K. M., T. Sakaki, A. Limsuwat. and T. H. Illangasekare. 2010. “Thermal Conductivity of Sands under Varying Moisture and Porosity in Drainage-Wetting cycles." Vadose Zone Journal 9: 172-80. https://doi.org/10.2136/vzj2009.0095.

Snijders, T. A. B., and R. J. Bosker. 1999. Multilevel Analysis: An Introduction to Basic and Advanced Multilevel Modeling. Thousand Oaks, CA: SAGE Publishing.

Sobol, I. M. 2001. "Global Sensitivity Indices for Nonlinear Mathematical Models and Their Monte Carlo Estimates.” Mathematics and Computers in Simulation 55 (13): 271-80.

Štrumbelj, E., and I. Kononenko. 2014. "Explaining Prediction Models and Individual Predictions with Feature Contributions." Knowledge and Information Systems 41 (3): 647-65.

Tavares, Z., J. Burroni, E. Minaysan, A. S. Lezama, and R. Ranganath. 2019a. Soft Constraints for Inference with Declarative Knowledge. arXiv preprint. Ithaca, NY: Cornell University. https://arxiv.org/abs/1901.05437v1.

Tavares, Z., J. Burroni, E. Minaysan, R. Ranganath, and A. S. Lezama. 2019b. The Random Conditional Distribution for Higher-Order Probabilistic Inference. arXiv. Preprint. Ithaca, NY: Cornell University. https://arxiv.org/abs/1903.10556v1. 
Tavares, Z., X. Zhang, J. Koppel, and A. S. Lezama. 2019c. Soft Constraints for Inference with Declarative Knowledge. arXiv. Preprint. Ithaca, NY: Cornell University. https://arxiv.org/abs/1901.05437v1.

Teichgraeber, H., L. E. Kuepper, and A. R. Brandt. 2019. "Time Series Clustering: An Extensible Framework in Julia.” Journal of Open Source Software 4 (41): 1573.

Thành, N. T., H. Sahli, and D. N. Hào. 2011. "Detection and Characterization of Buried Landmines Using Infrared Thermography." Inverse Problems in Science and Engineering 19 (3): 281-307.

Trautz, A., R. Kala, S. E. Howington, M. Bray, and M. W. Farthing. 2021 (In Press). Model Development, Calibration, and Validation for Support of Remote Thermal Detection of Buried Objects Research. ERDC/GSL xx-2021. Vicksburg, MS: U.S. Army Engineer Research and Development Center, Coastal and Hydraulics Laboratory.

Usher, W., J. Herman, C. Whealton, D. Hadka, Xantares, F. Rios, Bernardoct, C. Mutel, and J. van Engelen. 2016. "SALib/SALib: Launch!" Accessed May 2020. https://zenodo.org/record/160164.

van Dam, R. L., B. Borchers, J. M. H. Hendrickx, and S. Hong. 2003. "Soil Effects on Thermal Signatures of Buried Nonmetallic Landmines." Proceedings of SPIE 5089, Detection and Remediation Technologies for Mines and Minelike Targets VIII. https://doi.org/10.1117/12.487205.

van De Griend, A. A., P. J. Camillo, and R. J. Gurney. 1985. "Discrimination of Soil Physical Parameters, Thermal Inertia, and Soil Moisture from Diurnal Surface Temperature Fluctuations." Water Resources Research 21 (7): 997-1009.

von Deimling, J. S., P. Held, P. Feldens, and D. Wilken. 2015. "Effects of Using Inclined Parametric Echo Sounding on Sub-Bottom Acoustic Imaging and Advances in Buried Object Detection.“ Geo-Marine Letters 36: 113-19. https://doi.org/10.1007/s00367-015-0433-3.

Waldemar, S., P. Hlosta, J. Jarzemski, L. Szugajew, and J. Usowicz. 2012. "Role of Moisture and Density of Sand for Microwave Enhancement of Thermal Detection of Buried Mines." In Proceedings of SPIE 8357, Detection and Sensing of Mines, Explosive Objects, and Obscured Targets XVII. https://doi.org/10.1117/12.921335.

Wasserman, L. 2020. Optimal Transport and Wasserstein Distance. Accessed December 2020. http://www.stat.cmu.edu/ larry/=sml/0pt.pdf.

Weisberg, S. 2002. "Dimension Reduction Regression in R.” Journal of Statistical Software 7 (1): 1-22.

XGBoost Open Source Community. 2020. "Xgboost Parameters." Accessed December 2020. https://xgboost.readthedocs.io/en/latest/parameter.html.

Yao, Y., M. Wen, and Y. Wang. 2019. "Multi-Temporal IR Thermography for Mine Detection." 2019 1oth International Workshop on the Analysis of Multitemporal Remote Sensing Images (MultiTemp), 1-4. https://doi.org/10.1109/MultiTemp.2019.8866906. 
Zou, K. H., A. J. O’Malley, and L. Mauri. 2007. “Receiver-Operating Characteristic Analysis for Evaluating Diagnostic Tests and Predictive Models. Circulation 115 (5): 654-57.

Zou, K. H., L. Ohno-Machado, A. I. Bandos, H. E. Rockette, and A. Liu. 2011. Statistical Evaluation of Diagnostic Performance: Topics in ROC Analysis. First edition. London: Chapman and Hall/CRC. https://doi.org/10.1201/b11031. 


\section{Appendix}

\section{Appendix A}

Table A-1. Descriptive statistics for dependent and independent variables of point layout datasets for shallow and deep metal contrasts for timeline 15 July-31 August 2018.

\begin{tabular}{|c|c|c|c|c|c|c|c|c|c|c|c|}
\hline \multirow{3}{*}{$\begin{array}{l}\text { Contrast } \\
\text { Probe_id }\end{array}$} & \multirow{3}{*}{$\begin{array}{l}- \\
- \\
N\end{array}$} & \multicolumn{5}{|c|}{ Shallow Metal } & \multicolumn{5}{|c|}{ Deep Metal } \\
\hline & & \multicolumn{5}{|c|}{$1-10,12,14,19,28$} & \multicolumn{5}{|c|}{$1-10,11,12,14,28$} \\
\hline & & Min & Max & Mean & SD & $\begin{array}{l}\text { SE } \\
\text { Mean }\end{array}$ & Min & Max & Mean & SD & $\begin{array}{l}\text { SE } \\
\text { Mean }\end{array}$ \\
\hline Object & 16128 & 0 & 1 & 0.07 & 0.25 & 0 & 0 & 1 & 0.07 & 0.25 & 0 \\
\hline Time & 16128 & 1 & 24 & 12.5 & 6.9 & 0.05 & 1 & 24 & 12.5 & 6.9 & 0.05 \\
\hline AirTemp & 16128 & 8.33 & 33.56 & 21.57 & 4.52 & 0.04 & 8.33 & 33.56 & 21.57 & 4.52 & 0.04 \\
\hline $\mathrm{Rh}$ & 16128 & 23.70 & 98.10 & 79.64 & 18.33 & 0.14 & 23.70 & 98.10 & 79.64 & 18.33 & 0.14 \\
\hline WndSpd & 16128 & 0.02 & 3.09 & 0.79 & 0.57 & 0.00 & 0.02 & 3.09 & 0.79 & 0.57 & 0.00 \\
\hline WndDir & 16128 & 34.50 & 306.18 & 177.75 & 58.06 & 0.46 & 34.50 & 306.18 & 177.75 & 58.06 & 0.46 \\
\hline Rain & 16128 & 0.00 & 11.43 & 0.05 & 0.40 & 0.00 & 0.00 & 11.43 & 0.05 & 0.40 & 0.00 \\
\hline SW & 16128 & -9.32 & 966.5 & 197.06 & 267.5 & 2.11 & -9.32 & 966.5 & 197.06 & 267.5 & 2.11 \\
\hline SWR & 16128 & -6.48 & 148.05 & 28.07 & 38.01 & 0.3 & -6.48 & 148.05 & 28.07 & 38.01 & 0.3 \\
\hline SoilTemp5 & 16128 & 15.17 & 42.85 & 25.74 & 4.73 & 0.04 & 20.25 & 32.68 & 25.52 & 1.84 & 0.01 \\
\hline SoilTemp5 Time1 & 672 & 17.13 & 29.88 & 23.08 & 2.01 & 0.08 & 22.94 & 31.67 & 26.21 & 1.73 & 0.07 \\
\hline SoilTemp5 Time2 & 672 & 16.66 & 29.08 & 22.56 & 2.01 & 0.08 & 22.61 & 31.32 & 25.96 & 1.67 & 0.06 \\
\hline SoilTemp5 Time3 & 672 & 16.22 & 28.53 & 22.14 & 2.03 & 0.08 & 22.25 & 30.99 & 25.70 & 1.63 & 0.06 \\
\hline SoilTemp5 Time4 & 672 & 15.88 & 28.02 & 21.79 & 2.05 & 0.08 & 21.94 & 30.63 & 25.45 & 1.58 & 0.06 \\
\hline SoilTemp5 Time5 & 672 & 15.58 & 27.78 & 21.48 & 2.09 & 0.08 & 21.61 & 30.33 & 25.20 & 1.54 & 0.06 \\
\hline SoilTemp5 Time6 & 672 & 15.17 & 27.52 & 21.22 & 2.13 & 0.08 & 21.30 & 30.03 & 24.97 & 1.51 & 0.06 \\
\hline SoilTemp5 Time 7 & 672 & 15.18 & 27.35 & 21.08 & 2.15 & 0.08 & 21.00 & 29.78 & 24.74 & 1.49 & 0.06 \\
\hline SoilTemp5 Time8 & 672 & 15.55 & 27.14 & 21.43 & 2.14 & 0.08 & 20.72 & 29.55 & 24.53 & 1.47 & 0.06 \\
\hline SoilTemp5 Time9 & 672 & 16.74 & 26.59 & 22.41 & 2.08 & 0.08 & 20.47 & 29.35 & 24.35 & 1.46 & 0.06 \\
\hline SoilTemp5 Time10 & 672 & 19.08 & 28.96 & 23.91 & 2.15 & 0.08 & 20.31 & 29.16 & 24.23 & 1.46 & 0.06 \\
\hline SoilTemp5 Time11 & 672 & 20.22 & 32.27 & 25.83 & 2.41 & 0.09 & 20.25 & 28.91 & 24.20 & 1.45 & 0.06 \\
\hline SoilTemp5 Time12 & 672 & 21.10 & 35.55 & 27.85 & 2.91 & 0.11 & 20.36 & 28.71 & 24.29 & 1.44 & 0.06 \\
\hline SoilTemp5 Time13 & 672 & 22.03 & 38.36 & 29.64 & 3.28 & 0.13 & 20.64 & 28.47 & 24.50 & 1.43 & 0.06 \\
\hline SoilTemp5 Time14 & 672 & 22.72 & 40.92 & 30.97 & 3.68 & 0.14 & 21.08 & 28.30 & 24.82 & 1.42 & 0.05 \\
\hline SoilTemp5 Time15 & 672 & 23.66 & 42.63 & 31.62 & 4.07 & 0.16 & 21.66 & 29.00 & 25.22 & 1.44 & 0.06 \\
\hline SoilTemp5 Time16 & 672 & 24.47 & 42.86 & 31.70 & 4.24 & 0.16 & 22.27 & 29.89 & 25.65 & 1.49 & 0.06 \\
\hline SoilTemp5 Time17 & 672 & 24.58 & 42.59 & 31.36 & 4.33 & 0.17 & 22.63 & 30.75 & 26.05 & 1.57 & 0.06 \\
\hline SoilTemp5 Time18 & 672 & 24.00 & 41.92 & 30.61 & 4.19 & 0.17 & 23.00 & 31.47 & 26.39 & 1.65 & 0.06 \\
\hline SoilTemp5 Time19 & 672 & 23.38 & 39.97 & 29.51 & 3.80 & 0.16 & 23.38 & 32.07 & 26.65 & 1.73 & 0.07 \\
\hline SoilTemp5 Time20 & 672 & 22.86 & 37.66 & 27.97 & 3.23 & 0.15 & 23.47 & 32.45 & 26.81 & 1.80 & 0.07 \\
\hline SoilTemp5 Time21 & 672 & 20.88 & 35.33 & 26.44 & 2.74 & 0.12 & 23.50 & 32.63 & 26.86 & 1.84 & 0.07 \\
\hline
\end{tabular}




\begin{tabular}{|c|c|c|c|c|c|c|c|c|c|c|c|}
\hline Contrast & \multirow{3}{*}{$\begin{array}{l}- \\
- \\
N\end{array}$} & \multicolumn{5}{|c|}{ Shallow Metal } & \multicolumn{5}{|c|}{ Deep Metal } \\
\hline \multirow{2}{*}{ Probe_id } & & \multicolumn{5}{|c|}{$1-10,12,14,19,28$} & \multicolumn{5}{|c|}{$1-10,11,12,14,28$} \\
\hline & & Min & Max & Mean & SD & $\begin{array}{l}\text { SE } \\
\text { Mean }\end{array}$ & Min & Max & Mean & SD & $\begin{array}{l}\text { SE } \\
\text { Mean }\end{array}$ \\
\hline SoilTemp5 Time22 & 672 & 19.44 & 33.35 & 25.25 & 2.42 & 0.11 & 23.50 & 32.56 & 26.79 & 1.85 & 0.07 \\
\hline SoilTemp5 Time23 & 672 & 18.45 & 31.91 & 24.34 & 2.22 & 0.09 & 23.46 & 32.35 & 26.64 & 1.83 & 0.07 \\
\hline SoilTemp5 Time24 & 672 & 17.72 & 30.82 & 23.62 & 2.09 & 0.08 & 23.25 & 32.02 & 26.42 & 1.79 & 0.07 \\
\hline
\end{tabular}


Table A-2. Descriptive statistics for dependent and independent variables of point layout datasets for shallow and deep plastic contrasts for timeline 15 July-31 August 2018.

\begin{tabular}{|c|c|c|c|c|c|c|c|c|c|c|c|}
\hline Contrast & - & Shallon & Plastic & & & & Deep P & astic & & & \\
\hline Probe_id & - & $1-10,1$ & $2,13,14$, & & & & $1-10,1$ & $2,14,28$ & nd 33 & & \\
\hline & $N$ & Min & Max & Mean & SD & \begin{tabular}{|l|} 
SE \\
Mean
\end{tabular} & Min & Max & Mean & SD & $\begin{array}{l}\text { SE } \\
\text { Mean }\end{array}$ \\
\hline Object & 16128 & 0 & 1 & 0.07 & 0.25 & 0 & 0 & 1 & 0.07 & 0.25 & 0 \\
\hline Time & 16128 & 1 & 24 & 12.5 & 6.9 & 0.05 & 1 & 24 & 12.5 & 6.9 & 0.05 \\
\hline AirTemp & 16128 & 8.33 & 33.56 & 21.57 & 4.52 & 0.04 & 8.33 & 33.56 & 21.57 & 4.52 & 0.04 \\
\hline $\mathrm{Rh}$ & 16128 & 23.70 & 98.10 & 79.64 & 18.33 & 0.14 & 23.70 & 98.10 & 79.64 & 18.33 & 0.14 \\
\hline WndSpd & 16128 & 0.02 & 3.09 & 0.79 & 0.57 & 0.00 & 0.02 & 3.09 & 0.79 & 0.57 & 0.00 \\
\hline WndDir & 16128 & 34.50 & 306.18 & 177.75 & 58.06 & 0.46 & 34.50 & 306.18 & 177.75 & 58.06 & 0.46 \\
\hline Rain & 16128 & 0.00 & 11.43 & 0.05 & 0.40 & 0.00 & 0.00 & 11.43 & 0.05 & 0.40 & 0.00 \\
\hline SW & 16128 & -9.32 & 966.5 & 197.06 & 267.5 & 2.11 & -9.32 & 966.5 & 197.06 & 267.5 & 2.11 \\
\hline SWR & 16128 & -6.48 & 148.05 & 28.07 & 38.01 & 0.3 & -6.48 & 148.05 & 28.07 & 38.01 & 0.3 \\
\hline SoilTemp5 & 16128 & 10.55 & 52.55 & 25.78 & 5.03 & 0.04 & 19.27 & 34.24 & 25.52 & 1.88 & 0.01 \\
\hline SoilTemp5 Time1 & 672 & 13.25 & 29.88 & 22.95 & 2.15 & 0.08 & 22.94 & 33.19 & 26.23 & 1.76 & 0.07 \\
\hline SoilTemp5 Time2 & 672 & 12.58 & 29.08 & 22.42 & 2.17 & 0.08 & 22.61 & 32.69 & 25.97 & 1.71 & 0.07 \\
\hline SoilTemp5 Time3 & 672 & 12.00 & 28.53 & 21.98 & 2.20 & 0.08 & 22.19 & 32.14 & 25.71 & 1.65 & 0.06 \\
\hline SoilTemp5 Time4 & 672 & 11.60 & 28.02 & 21.63 & 2.22 & 0.09 & 21.69 & 31.64 & 25.45 & 1.60 & 0.06 \\
\hline SoilTemp5 Time5 & 672 & 11.11 & 27.78 & 21.32 & 2.26 & 0.09 & 21.19 & 31.19 & 25.19 & 1.56 & 0.06 \\
\hline SoilTemp5 Time6 & 672 & 10.55 & 27.52 & 21.05 & 2.30 & 0.09 & 20.75 & 30.72 & 24.95 & 1.53 & 0.06 \\
\hline SoilTemp5 Time7 & 672 & 10.74 & 27.35 & 20.92 & 2.31 & 0.09 & 20.33 & 30.32 & 24.72 & 1.50 & 0.06 \\
\hline SoilTemp5 Time8 & 672 & 12.16 & 27.14 & 21.29 & 2.26 & 0.09 & 19.91 & 29.94 & 24.50 & 1.49 & 0.06 \\
\hline SoilTemp5 Time9 & 672 & 14.70 & 26.59 & 22.32 & 2.14 & 0.08 & 19.57 & 29.61 & 24.31 & 1.48 & 0.06 \\
\hline SoilTemp5 Time10 & 672 & 19.05 & 29.42 & 23.90 & 2.20 & 0.09 & 19.31 & 29.28 & 24.18 & 1.48 & 0.06 \\
\hline SoilTemp5 Time11 & 672 & 19.75 & 35.46 & 25.91 & 2.56 & 0.10 & 19.27 & 28.94 & 24.15 & 1.47 & 0.06 \\
\hline SoilTemp5 Time12 & 672 & 20.78 & 40.63 & 28.03 & 3.21 & 0.12 & 19.49 & 28.61 & 24.24 & 1.46 & 0.06 \\
\hline SoilTemp5 Time13 & 672 & 22.03 & 45.55 & 29.90 & 3.69 & 0.14 & 20.00 & 28.27 & 24.45 & 1.44 & 0.06 \\
\hline SoilTemp5 Time14 & 672 & 22.72 & 49.80 & 31.28 & 4.15 & 0.16 & 20.77 & 28.13 & 24.78 & 1.43 & 0.06 \\
\hline SoilTemp5 Time15 & 672 & 23.66 & 52.30 & 31.96 & 4.54 & 0.17 & 21.66 & 28.82 & 25.19 & 1.45 & 0.06 \\
\hline SoilTemp5 Time16 & 672 & 24.47 & 52.55 & 32.04 & 4.68 & 0.18 & 22.27 & 29.97 & 25.63 & 1.50 & 0.06 \\
\hline SoilTemp5 Time17 & 672 & 24.67 & 51.47 & 31.67 & 4.72 & 0.18 & 22.63 & 31.19 & 26.04 & 1.59 & 0.06 \\
\hline SoilTemp5 Time18 & 672 & 23.96 & 49.89 & 30.87 & 4.51 & 0.17 & 23.00 & 32.26 & 26.40 & 1.68 & 0.06 \\
\hline SoilTemp5 Time19 & 672 & 23.13 & 46.75 & 29.71 & 4.05 & 0.16 & 23.38 & 33.14 & 26.67 & 1.77 & 0.07 \\
\hline SoilTemp5 Time20 & 672 & 21.55 & 42.45 & 28.10 & 3.38 & 0.13 & 23.47 & 33.82 & 26.84 & 1.85 & 0.07 \\
\hline SoilTemp5 Time21 & 672 & 19.33 & 37.94 & 26.49 & 2.83 & 0.11 & 23.50 & 34.21 & 26.90 & 1.89 & 0.07 \\
\hline SoilTemp5 Time22 & 672 & 17.13 & 34.16 & 25.23 & 2.50 & 0.10 & 23.50 & 34.24 & 26.83 & 1.90 & 0.07 \\
\hline SoilTemp5 Time23 & 672 & 15.38 & 31.91 & 24.26 & 2.32 & 0.09 & 23.46 & 34.03 & 26.67 & 1.88 & 0.07 \\
\hline SoilTemp5 Time24 & 672 & 14.14 & 30.82 & 23.52 & 2.22 & 0.09 & 23.25 & 33.66 & 26.46 & 1.83 & 0.07 \\
\hline
\end{tabular}


Table A-3. Descriptive statistics of soil temperature observations at $25 \mathrm{~cm}$ for timeline 15 July-31 August 2018.

\begin{tabular}{|c|c|c|c|c|c|c|c|c|c|c|c|}
\hline \multirow{3}{*}{$\begin{array}{l}\text { Contrast } \\
\text { Probe_id }\end{array}$} & \multirow{3}{*}{-} & \multicolumn{5}{|c|}{ Shallow Metal } & \multicolumn{5}{|c|}{ Deep Metal } \\
\hline & & \multicolumn{5}{|c|}{$1-10,12,14,19,28$} & \multicolumn{5}{|c|}{$1-10,11,12,14,28$} \\
\hline & & Min & Max & Mean & SD & $\begin{array}{l}\text { SE } \\
\text { Mean }\end{array}$ & Min & Max & Mean & SD & $\begin{array}{l}\text { SE } \\
\text { Mean }\end{array}$ \\
\hline SoilTemp25cm & 16128 & 20.25 & 32.38 & 25.47 & 1.83 & 0.01 & 20.25 & 32.63 & 25.53 & 1.84 & 0.01 \\
\hline SoilTemp25cm Time1 & 672 & 22.50 & 31.49 & 26.14 & 1.72 & 0.07 & 22.94 & 31.67 & 26.21 & 1.73 & 0.07 \\
\hline SoilTemp25cm Time2 & 672 & 22.17 & 31.13 & 25.89 & 1.67 & 0.06 & 22.61 & 31.32 & 25.96 & 1.67 & 0.06 \\
\hline SoilTemp25cm Time3 & 672 & 21.86 & 30.77 & 25.63 & 1.62 & 0.06 & 22.25 & 30.99 & 25.70 & 1.63 & 0.06 \\
\hline SoilTemp25cm Time4 & 672 & 21.57 & 30.44 & 25.39 & 1.58 & 0.06 & 21.94 & 30.63 & 25.45 & 1.58 & 0.06 \\
\hline SoilTemp25cm Time5 & 672 & 21.28 & 30.11 & 25.14 & 1.54 & 0.06 & 21.61 & 30.33 & 25.20 & 1.54 & 0.06 \\
\hline SoilTemp25cm Time6 & 672 & 21.00 & 29.80 & 24.91 & 1.51 & 0.06 & 21.30 & 30.03 & 24.97 & 1.51 & 0.06 \\
\hline SoilTemp25cm Time7 & 672 & 20.72 & 29.53 & 24.68 & 1.48 & 0.06 & 21.00 & 29.78 & 24.74 & 1.49 & 0.06 \\
\hline SoilTemp25cm Time8 & 672 & 20.52 & 29.28 & 24.48 & 1.46 & 0.06 & 20.72 & 29.55 & 24.53 & 1.47 & 0.06 \\
\hline SoilTemp25cm Time9 & 672 & 20.40 & 29.08 & 24.30 & 1.45 & 0.06 & 20.47 & 29.35 & 24.35 & 1.46 & 0.06 \\
\hline SoilTemp25cm Time10 & 672 & 20.31 & 28.86 & 24.19 & 1.44 & 0.06 & 20.31 & 29.16 & 24.23 & 1.46 & 0.06 \\
\hline SoilTemp25cm Time11 & 672 & 20.25 & 28.66 & 24.17 & 1.44 & 0.06 & 20.25 & 28.91 & 24.20 & 1.45 & 0.06 \\
\hline SoilTemp25cm Time12 & 672 & 20.36 & 28.47 & 24.27 & 1.43 & 0.06 & 20.36 & 28.71 & 24.29 & 1.44 & 0.06 \\
\hline SoilTemp25cm Time13 & 672 & 20.64 & 28.27 & 24.49 & 1.41 & 0.05 & 20.64 & 28.47 & 24.50 & 1.43 & 0.06 \\
\hline SoilTemp25cm Time14 & 672 & 21.08 & 28.13 & 24.81 & 1.41 & 0.05 & 21.08 & 28.30 & 24.82 & 1.42 & 0.05 \\
\hline SoilTemp25cm Time15 & 672 & 21.66 & 28.72 & 25.20 & 1.43 & 0.06 & 21.66 & 29.00 & 25.22 & 1.44 & 0.06 \\
\hline SoilTemp25cm Time16 & 672 & 22.27 & 29.57 & 25.61 & 1.48 & 0.06 & 22.27 & 29.89 & 25.65 & 1.49 & 0.06 \\
\hline SoilTemp25cm Time17 & 672 & 22.63 & 30.42 & 26.00 & 1.55 & 0.06 & 22.63 & 30.75 & 26.05 & 1.57 & 0.06 \\
\hline SoilTemp25cm Time18 & 672 & 23.00 & 31.17 & 26.33 & 1.63 & 0.06 & 23.00 & 31.47 & 26.39 & 1.65 & 0.06 \\
\hline SoilTemp25cm Time19 & 672 & 23.38 & 31.77 & 26.58 & 1.72 & 0.07 & 23.38 & 32.07 & 26.65 & 1.73 & 0.07 \\
\hline SoilTemp25cm Time20 & 672 & 23.47 & 32.19 & 26.74 & 1.79 & 0.07 & 23.47 & 32.45 & 26.81 & 1.80 & 0.07 \\
\hline SoilTemp25cm Time21 & 672 & 23.50 & 32.38 & 26.78 & 1.83 & 0.07 & 23.50 & 32.63 & 26.86 & 1.84 & 0.07 \\
\hline SoilTemp25cm Time22 & 672 & 23.44 & 32.35 & 26.71 & 1.84 & 0.07 & 23.50 & 32.56 & 26.79 & 1.85 & 0.07 \\
\hline SoilTemp25cm Time23 & 672 & 23.13 & 32.14 & 26.56 & 1.82 & 0.07 & 23.46 & 32.35 & 26.64 & 1.83 & 0.07 \\
\hline SoilTemp25cm Time24 & 672 & 22.82 & 31.83 & 26.35 & 1.78 & 0.07 & 23.25 & 32.02 & 26.42 & 1.79 & 0.07 \\
\hline
\end{tabular}


Table A-4. Descriptive statistics of soil temperature observations at $25 \mathrm{~cm}$ for timeline 15 July15-31 August 2018.

\begin{tabular}{|c|c|c|c|c|c|c|c|c|c|c|c|}
\hline Contrast & \multirow[b]{3}{*}{$\mathbf{N}$} & \multicolumn{5}{|c|}{ Shallow Plastic } & \multicolumn{5}{|c|}{ Deep Plastic } \\
\hline \multirow{2}{*}{ Probe_id } & & \multicolumn{5}{|c|}{$1-10,12,13,14,28$} & \multicolumn{5}{|c|}{$1-10,12,14,28$ and 33} \\
\hline & & Min & Max & Mean & SD & $\begin{array}{l}\text { SE } \\
\text { Mean }\end{array}$ & Min & Max & Mean & SD & $\begin{array}{l}\text { SE } \\
\text { Mean }\end{array}$ \\
\hline SoilTemp25cm & 16128 & 20.25 & 32.38 & 25.49 & 1.80 & 0.01 & 19.27 & 34.23 & 25.52 & 1.88 & 0.01 \\
\hline SoilTemp25cm Time1 & 672 & 22.94 & 31.49 & 26.16 & 1.69 & 0.07 & 22.94 & 33.19 & 26.23 & 1.76 & 0.07 \\
\hline SoilTemp25cm Time2 & 672 & 22.61 & 31.13 & 25.93 & 1.64 & 0.06 & 22.61 & 32.69 & 25.97 & 1.71 & 0.07 \\
\hline SoilTemp25cm Time3 & 672 & 22.25 & 30.77 & 25.70 & 1.59 & 0.06 & 22.19 & 32.14 & 25.71 & 1.65 & 0.06 \\
\hline SoilTemp25cm Time4 & 672 & 21.94 & 30.44 & 25.46 & 1.56 & 0.06 & 21.69 & 31.64 & 25.45 & 1.60 & 0.06 \\
\hline SoilTemp25cm Time5 & 672 & 21.61 & 30.11 & 25.23 & 1.52 & 0.06 & 21.19 & 31.19 & 25.19 & 1.56 & 0.06 \\
\hline SoilTemp25cm Time6 & 672 & 21.30 & 29.80 & 25.01 & 1.50 & 0.06 & 20.75 & 30.72 & 24.95 & 1.53 & 0.06 \\
\hline SoilTemp25cm Time7 & 672 & 21.00 & 29.53 & 24.79 & 1.48 & 0.06 & 20.33 & 30.32 & 24.72 & 1.50 & 0.06 \\
\hline SoilTemp25cm Time8 & 672 & 20.72 & 29.28 & 24.59 & 1.47 & 0.06 & 19.91 & 29.94 & 24.50 & 1.49 & 0.06 \\
\hline SoilTemp25cm Time9 & 672 & 20.47 & 29.08 & 24.41 & 1.46 & 0.06 & 19.57 & 29.61 & 24.31 & 1.48 & 0.06 \\
\hline SoilTemp25cm Time10 & 672 & 20.31 & 28.86 & 24.29 & 1.46 & 0.06 & 19.31 & 29.28 & 24.18 & 1.48 & 0.06 \\
\hline SoilTemp25cm Time11 & 672 & 20.25 & 28.66 & 24.25 & 1.45 & 0.06 & 19.27 & 28.94 & 24.15 & 1.47 & 0.06 \\
\hline SoilTemp25cm Time12 & 672 & 20.36 & 28.53 & 24.32 & 1.43 & 0.06 & 19.49 & 28.61 & 24.24 & 1.46 & 0.06 \\
\hline SoilTemp25cm Time13 & 672 & 20.64 & 28.41 & 24.51 & 1.41 & 0.05 & 20.00 & 28.27 & 24.45 & 1.44 & 0.06 \\
\hline SoilTemp25cm Time14 & 672 & 21.08 & 28.30 & 24.80 & 1.40 & 0.05 & 20.77 & 28.13 & 24.78 & 1.43 & 0.06 \\
\hline SoilTemp25cm Time15 & 672 & 21.66 & 28.69 & 25.16 & 1.42 & 0.05 & 21.66 & 28.82 & 25.19 & 1.45 & 0.06 \\
\hline SoilTemp25cm Time16 & 672 & 21.94 & 29.57 & 25.55 & 1.48 & 0.06 & 22.27 & 29.97 & 25.63 & 1.50 & 0.06 \\
\hline SoilTemp25cm Time17 & 672 & 21.99 & 30.42 & 25.93 & 1.56 & 0.06 & 22.63 & 31.19 & 26.04 & 1.59 & 0.06 \\
\hline SoilTemp25cm Time18 & 672 & 22.13 & 31.17 & 26.25 & 1.65 & 0.06 & 23.00 & 32.26 & 26.40 & 1.68 & 0.06 \\
\hline SoilTemp25cm Time19 & 672 & 22.28 & 31.77 & 26.50 & 1.73 & 0.07 & 23.38 & 33.14 & 26.67 & 1.77 & 0.07 \\
\hline SoilTemp25cm Time20 & 672 & 22.49 & 32.19 & 26.66 & 1.80 & 0.07 & 23.47 & 33.82 & 26.84 & 1.85 & 0.07 \\
\hline SoilTemp25cm Time21 & 672 & 22.71 & 32.38 & 26.72 & 1.83 & 0.07 & 23.50 & 34.21 & 26.90 & 1.89 & 0.07 \\
\hline SoilTemp25cm Time22 & 672 & 22.89 & 32.35 & 26.67 & 1.83 & 0.07 & 23.50 & 34.24 & 83 & 1.90 & 0.07 \\
\hline SoilTemp25cm Time23 & 672 & 23.08 & 32.14 & 26.54 & 1.80 & 0.07 & 23.46 & 34.03 & 26.67 & 1.88 & 0.07 \\
\hline SoilTemp25cm Time24 & 672 & 23.22 & 31.83 & 26.35 & 1.76 & 0.07 & 23.25 & 33.66 & 26.46 & 1.83 & 0.07 \\
\hline
\end{tabular}


Table A-5. Descriptive statistics of IR temperatures above the shallow and deep metal contrasts for timeline 15 July-31 August 2018.

\begin{tabular}{|c|c|c|c|c|c|c|c|c|c|c|c|}
\hline \multirow{3}{*}{$\begin{array}{l}\text { Contrast } \\
\text { Probe_id }\end{array}$} & \multirow[b]{3}{*}{$\Lambda$} & \multicolumn{5}{|c|}{ Shallow Metal } & \multicolumn{5}{|c|}{ Deep Metal } \\
\hline & & \multicolumn{5}{|c|}{$1,10,12,14,19,28$} & \multicolumn{5}{|c|}{$1,10,11,12,14,28$} \\
\hline & & Min & Max & Mean & SD & $\begin{array}{l}\mathrm{SE} \\
\text { Mean }\end{array}$ & Min & Max & Mean & SD & $\begin{array}{l}\text { SE } \\
\text { Mean }\end{array}$ \\
\hline Object & 691200 & 0 & 1 & 0.17 & .37 & 0 & 0 & 1 & 0.17 & 0.37 & 0 \\
\hline IR_tir & 1200 & 8.06 & 59.58 & 26.35 & 29 & 0.01 & 8.38 & 60.98 & 6.36 & 7.27 & 0.01 \\
\hline IR_tir Time1 & 8800 & 11.37 & 27.38 & 20.88 & 3.32 & 0.02 & 12.22 & 27.38 & 20.92 & 3.30 & 0.02 \\
\hline IR_tir Time2 & 28800 & 10.36 & 27.06 & 20.46 & 3.47 & 0.02 & 11.14 & 27.06 & 20.49 & 3.46 & 0.02 \\
\hline IR_tir Time3 & 28800 & 9.57 & 27.24 & 20.25 & 3.57 & 0.02 & 10.23 & 27.24 & 20.27 & 3.56 & 0.02 \\
\hline IR_tir Time4 & 28800 & 8.73 & 27.39 & 20.06 & 3.64 & 0.02 & 9.33 & 27.39 & 20.07 & 3.64 & 0.02 \\
\hline IR_tir Time5 & 28800 & 8.28 & 27.69 & 89 & 7 & 22 & 8.74 & 7.69 & 19.90 & 3.66 & 0.02 \\
\hline IR_tir Time6 & 300 & 8.06 & 38 & 76 & 74 & 0.02 & 8.38 & 7.38 & 9.76 & 3.74 & 0.02 \\
\hline IR_tir Time7 & 28800 & 9.87 & 27.66 & 11 & 57 & 0.02 & 10.11 & 27.78 & 0.10 & 3.58 & 0.02 \\
\hline IR_tir Time8 & 28800 & 13.65 & 11 & 5 & 17 & 0.02 & 13.56 & 7.11 & 64 & 3.19 & 0.02 \\
\hline IR_tir & 288 & 17.04 & 32.18 & 24.19 & 3.04 & 0.02 & 17.04 & 33.16 & 4.17 & 3.06 & 02 \\
\hline IR_ti & 2 & 2 & 8 & 4 & 7 & 2 & 59 & 37.06 & 9 & 3.36 & 0.02 \\
\hline IR_tir Time11 & 28800 & 21.09 & 44.67 & 30.34 & 2 & 3 & 2 & 46.53 & 26 & 4.31 & 0.03 \\
\hline IR_tir Time12 & 28800 & 22.64 & 51.18 & 33.22 & 5.01 & 0.03 & 22.60 & 52.91 & 33.13 & 4.99 & 0.03 \\
\hline IR_tir Time13 & 28800 & 23.88 & 55.95 & 35.25 & 5.53 & 0.03 & 23.85 & 57.82 & 35.16 & 5.53 & 0.03 \\
\hline IR_tir Time14 & 28800 & 25.40 & 59.58 & 36.07 & 5.91 & 0.03 & 25.40 & 60.98 & 35.99 & 5.90 & 0.03 \\
\hline IR_tir Time15 & 28800 & 25.81 & 59.53 & 35.92 & 6.20 & 0.04 & 25.81 & 60.31 & 35.87 & 6.19 & 0.04 \\
\hline IR_tir Time16 & 28800 & 25.99 & 6.24 & 5.07 & 6.07 & 0.04 & 25.99 & 57.25 & 35.07 & 6.08 & 0.04 \\
\hline IR_tir Time17 & 28800 & 24.72 & 54.94 & 33.80 & 5.85 & 0.03 & 24.72 & 55.60 & 33.83 & 5.89 & 0.03 \\
\hline IR_tir Time18 & 28800 & 23.71 & 50.08 & 31.88 & 5.04 & 0.03 & 23.71 & 50.73 & 31.93 & 5.09 & 0.03 \\
\hline IR_tir Time19 & 28800 & 21.46 & 42.71 & 29.55 & 3.99 & 0.02 & 21.46 & 43.49 & 29.62 & 4.04 & 0.02 \\
\hline IR_tir Time20 & 28800 & 18.85 & 35.79 & 26.48 & 2.96 & 0.02 & 19.20 & 35.79 & 26.56 & 2.98 & 0.02 \\
\hline IR_tir Time21 & 28800 & 16.22 & 32.19 & 24.27 & 2.74 & 0.02 & 16.27 & 32.19 & 24.36 & 2.74 & 0.02 \\
\hline IR_tir Time22 & 28800 & 14.49 & 29.89 & 22.82 & 2.83 & 0.02 & 14.57 & 29.89 & 22.91 & 2.82 & 0.02 \\
\hline IR_tir Time23 & 28800 & 13.45 & 29.41 & 21.99 & 3.03 & 0.02 & 13.56 & 29.41 & 22.06 & 3.01 & 0.02 \\
\hline IR_tir Time24 & 28800 & 12.45 & 28.32 & 21.39 & 3.10 & 0.02 & 12.96 & 28.32 & 21.44 & 3.08 & 0.02 \\
\hline
\end{tabular}


Table A-6. Descriptive statistics of IR temperatures above the shallow and deep plastic contrasts for timeline 15 July-31 August 2018.

\begin{tabular}{|c|c|c|c|c|c|c|c|c|c|c|c|}
\hline \multirow{3}{*}{$\begin{array}{l}\text { Contrast } \\
\text { Probe_id }\end{array}$} & \multirow{3}{*}{-} & \multicolumn{5}{|c|}{ Shallow Plastic } & \multicolumn{5}{|c|}{ Deep Plastic } \\
\hline & & \multicolumn{5}{|c|}{$1,10,12,13,14,28$} & \multicolumn{5}{|c|}{$1,10,12,14,28$ and 33} \\
\hline & & Min & Max & Mean & SD & $\begin{array}{l}\text { SE } \\
\text { Mean }\end{array}$ & Min & Max & Mean & SD & $\begin{array}{l}\text { SE } \\
\text { Mean }\end{array}$ \\
\hline Object & 691200 & 0 & 1 & 0.17 & 0.37 & 0 & 0 & 1 & 0.17 & 0.37 & 0 \\
\hline IR_tir & 691200 & 5.45 & 62.45 & 26.37 & 7.55 & 0.01 & 8.55 & 60.36 & 26.36 & 7.26 & 0.01 \\
\hline IR_tir Time1 & 28800 & 9.43 & 27.38 & 20.71 & 3.45 & 0.02 & 12.30 & 27.38 & 20.94 & 3.30 & 0.02 \\
\hline IR_tir Time2 & 28800 & 8.18 & 27.06 & 20.28 & 3.62 & 0.02 & 11.31 & 27.06 & 20.52 & 3.45 & 0.02 \\
\hline IR_tir Time3 & 28800 & 7.22 & 27.24 & 20.06 & 3.72 & 0.02 & 10.42 & 27.24 & 20.29 & 3.55 & 0.02 \\
\hline IR_tir Time4 & 28800 & 6.22 & 27.39 & 19.87 & 3.79 & 0.02 & 9.45 & 27.40 & 20.09 & 3.63 & 0.02 \\
\hline IR_tir Time5 & 28800 & 5.75 & 27.69 & 19.70 & 3.81 & 0.02 & 8.97 & 27.78 & 19.92 & 3.66 & 0.02 \\
\hline IR_tir Time6 & 28800 & 5.45 & 27.38 & 19.57 & 3.88 & 0.02 & 8.55 & 27.48 & 19.78 & 3.73 & 0.02 \\
\hline IR_tir Time7 & 28800 & 7.41 & 27.66 & 19.92 & 3.70 & 0.02 & 10.29 & 27.88 & 20.11 & 3.58 & 0.02 \\
\hline IR_tir Time8 & 28800 & 11.62 & 27.11 & 21.49 & 3.26 & 0.02 & 13.65 & 27.11 & 21.64 & 3.18 & 0.02 \\
\hline IR_tir Time9 & 28800 & 16.00 & 31.74 & 24.09 & 3.09 & 0.02 & 17.04 & 32.84 & 24.16 & 3.05 & 0.02 \\
\hline IR_tir Time10 & 28800 & 20.30 & 37.20 & 27.02 & 3.43 & 0.02 & 20.51 & 36.12 & 26.98 & 3.35 & 0.02 \\
\hline IR_tir Time11 & 28800 & 20.74 & 45.90 & 30.40 & 4.43 & 0.03 & 20.93 & 45.67 & 30.25 & 4.29 & 0.03 \\
\hline IR_tir Time12 & 28800 & 22.45 & 53.09 & 33.38 & 5.21 & 0.03 & 22.46 & 52.23 & 33.12 & 4.97 & 0.03 \\
\hline IR_tir Time13 & 28800 & 23.88 & 58.62 & 35.50 & 5.78 & 0.03 & 23.61 & 56.97 & 35.15 & 5.50 & 0.03 \\
\hline IR_tir Time14 & 28800 & 25.40 & 62.36 & 36.37 & 6.19 & 0.04 & 25.12 & 60.21 & 35.98 & 5.87 & 0.03 \\
\hline IR_tir Time15 & 28800 & 25.81 & 62.45 & 36.25 & 6.51 & 0.04 & 25.81 & 60.36 & 35.86 & 6.17 & 0.04 \\
\hline IR_tir Time16 & 28800 & 25.99 & 59.13 & 35.42 & 6.37 & 0.04 & 25.99 & 57.19 & 35.06 & 6.06 & 0.04 \\
\hline IR_tir Time17 & 28800 & 24.72 & 57.77 & 34.12 & 6.13 & 0.04 & 24.72 & 55.75 & 33.82 & 5.87 & 0.03 \\
\hline IR_tir Time18 & 28800 & 23.71 & 52.88 & 32.17 & 5.29 & 0.03 & 23.70 & 51.36 & 31.94 & 5.09 & 0.03 \\
\hline IR_tir Time19 & 28800 & 20.81 & 45.46 & 29.77 & 4.19 & 0.02 & 21.46 & 43.79 & 29.62 & 4.04 & 0.02 \\
\hline IR_tir Time20 & 28800 & 17.98 & 37.66 & 26.63 & 3.08 & 0.02 & 19.20 & 35.79 & 26.57 & 2.98 & 0.02 \\
\hline IR_tir Time21 & 28800 & 15.14 & 32.61 & 24.32 & 2.81 & 0.02 & 16.27 & 32.19 & 24.37 & 2.74 & 0.02 \\
\hline IR_tir Time22 & 28800 & 12.85 & 29.89 & 22.79 & 2.91 & 0.02 & 14.57 & 29.89 & 22.92 & 2.82 & 0.02 \\
\hline IR_tir Time23 & 28800 & 11.31 & 29.41 & 21.90 & 3.12 & 0.02 & 13.56 & 29.41 & 22.07 & 3.00 & 0.02 \\
\hline IR_tir Time24 & 28800 & 14.14 & 30.82 & 23.52 & 2.22 & 0.09 & 12.96 & 28.32 & 21.46 & 3.07 & 0.02 \\
\hline
\end{tabular}


Table A-7. Descriptive statistics of soil moisture observations at $15 \mathrm{~cm}$ for timeline 15 July31 August 2018.

\begin{tabular}{|c|c|c|c|c|c|c|c|c|c|c|c|}
\hline \multirow{3}{*}{$\begin{array}{l}\text { Contrast } \\
\text { Probe_id } \\
\end{array}$} & \multirow{3}{*}{\begin{tabular}{|l}
- \\
- \\
$N$
\end{tabular}} & \multicolumn{5}{|c|}{ Shallow Metal } & \multicolumn{5}{|c|}{ Deep Metal } \\
\hline & & \multicolumn{5}{|c|}{$1,10,12,14,19,28$} & \multicolumn{5}{|c|}{$1,10,11,12,14,28$} \\
\hline & & Min & Max & Mean & SD & \begin{tabular}{|l} 
SE \\
Mean
\end{tabular} & Min & Max & Mean & SD & $\begin{array}{l}\text { SE } \\
\text { Mean }\end{array}$ \\
\hline Soil $15 \mathrm{~cm}$ & - & 0.13 & 0.28 & 0.18 & 0.03 & 0 & 0.07 & 0.26 & 0.17 & 0.03 & 0 \\
\hline \begin{tabular}{|l|} 
Soil15cm \\
Time1
\end{tabular} & & 0.13 & 0.26 & 0.18 & 0.02 & 0 & 0.07 & 0.23 & 0.17 & 0.03 & 0 \\
\hline $\begin{array}{l}\text { Soil15cm } \\
\text { Time2 }\end{array}$ & & 0.13 & 0.26 & 0.18 & 0.03 & 0 & 0.07 & 0.24 & 0.17 & 0.03 & 0 \\
\hline $\begin{array}{l}\text { Soil15cm } \\
\text { Time3 }\end{array}$ & . & 0.13 & 0.27 & 0.18 & 0.03 & 0 & 0.07 & 0.25 & 0.17 & 0.03 & 0 \\
\hline \begin{tabular}{|l|} 
Soil15cm \\
Time4
\end{tabular} & - & 0.13 & 0.28 & 0.18 & 0.03 & 0 & 0.07 & 0.26 & 0.17 & 0.03 & 0 \\
\hline $\begin{array}{l}\text { Soil15cm } \\
\text { Time5 }\end{array}$ & $\left.\right|^{-}$ & 0.13 & 0.28 & 0.18 & 0.03 & 0 & 0.07 & 0.26 & 0.17 & 0.03 & 0 \\
\hline \begin{tabular}{|l|} 
Soil15cm \\
Time6
\end{tabular} & $\left.\right|^{-}$ & 0.13 & 0.28 & 0.18 & 0.03 & 0 & 0.07 & 0.26 & 0.17 & 0.03 & 0 \\
\hline \begin{tabular}{|l|} 
Soil15cm \\
Time7
\end{tabular} &  & 0.13 & 0.28 & 0.18 & 0.03 & 0 & 0.07 & 0.26 & 0.17 & 0.03 & 0 \\
\hline \begin{tabular}{|l|} 
Soil15cm \\
Time8
\end{tabular} & & 0.13 & 0.27 & 0.18 & 0.03 & 0 & 0.07 & 0.25 & 0.17 & 0.03 & 0 \\
\hline $\begin{array}{l}\text { Soil15cm } \\
\text { Time9 }\end{array}$ & [- & 0.13 & 0.27 & 0.18 & 0.03 & 0 & 0.07 & 0.25 & 0.17 & 0.03 & 0 \\
\hline \begin{tabular}{|l|} 
Soil15cm \\
Time10
\end{tabular} & - & 0.13 & 0.27 & 0.18 & 0.03 & 0 & 0.07 & 0.25 & 0.17 & 0.03 & 0 \\
\hline $\begin{array}{l}\text { Soil15cm } \\
\text { Time11 }\end{array}$ & - & 0.13 & 0.27 & 0.18 & 0.03 & 0 & 0.07 & 0.25 & 0.17 & 0.03 & 0 \\
\hline \begin{tabular}{|l|} 
Soil15cm \\
Time12 \\
\end{tabular} & - & 0.13 & 0.26 & 0.18 & 0.03 & 0 & 0.07 & 0.25 & 0.17 & 0.03 & 0 \\
\hline \begin{tabular}{|l|} 
Soil15cm \\
Time13
\end{tabular} & - & 0.13 & 0.26 & 0.18 & 0.03 & 0 & 0.08 & 0.24 & 0.17 & 0.03 & 0 \\
\hline \begin{tabular}{|l|} 
Soil15cm \\
Time14
\end{tabular} & - & 0.13 & 0.26 & 0.18 & 0.03 & 0 & 0.08 & 0.24 & 0.17 & 0.03 & 0 \\
\hline \begin{tabular}{|l} 
Soil15cm \\
Time15
\end{tabular} & - & 0.13 & 0.26 & 0.18 & 0.02 & 0 & 0.08 & \begin{tabular}{|l|}
0.25 \\
\end{tabular} & 0.17 & 0.03 & 0 \\
\hline \begin{tabular}{|l|} 
Soil15cm \\
Time16
\end{tabular} & $\left.\right|^{-}$ & 0.13 & 0.26 & 0.18 & 0.02 & 0 & 0.08 & 0.25 & 0.17 & 0.03 & 0 \\
\hline \begin{tabular}{|l|} 
Soil15cm \\
Time17
\end{tabular} & - & 0.13 & 0.26 & 0.18 & 0.02 & 0 & 0.08 & 0.24 & 0.17 & 0.03 & 0 \\
\hline $\begin{array}{l}\text { Soil15cm } \\
\text { Time18 }\end{array}$ & {[} & 0.13 & 0.27 & 0.18 & 0.02 & 0 & 0.08 & 0.24 & 0.17 & 0.03 & 0 \\
\hline \begin{tabular}{|l|} 
Soil15cm \\
Time19 \\
\end{tabular} & & 0.13 & 0.27 & 0.18 & 0.02 & 0 & 0.08 & 0.24 & 0.17 & 0.03 & 0 \\
\hline $\begin{array}{l}\text { Soil15cm } \\
\text { Time20 }\end{array}$ & & 0.13 & 0.27 & 0.18 & 0.02 & 0 & 0.08 & \begin{tabular}{|l|} 
\\
\end{tabular} & 0.17 & 0.03 & 0 \\
\hline
\end{tabular}




\begin{tabular}{|l|l|l|l|l|l|l|l|l|l|l|l|}
\hline Contrast & - & \multicolumn{4}{|l|}{ Shallow Metal } & \multicolumn{4}{|l|}{ Deep Metal } \\
\hline Probe_id & - & \multicolumn{1}{|l}{$1,10,12,14,19,28$} & \multicolumn{1}{l|}{$1,12,14,28$} \\
\hline & N & Min & Max & Mean & SD & $\begin{array}{l}\text { SE } \\
\text { Mean }\end{array}$ & Min & Max & Mean & SD & $\begin{array}{l}\text { SE } \\
\text { Mean }\end{array}$ \\
\hline $\begin{array}{l}\text { Soil15cm } \\
\text { Time21 }\end{array}$ & - & 0.13 & 0.26 & 0.18 & 0.02 & 0 & 0.08 & 0.24 & 0.17 & 0.03 & 0 \\
\hline $\begin{array}{l}\text { Soil15cm } \\
\text { Time22 }\end{array}$ & - & 0.13 & 0.26 & 0.18 & 0.02 & 0 & 0.08 & 0.23 & 0.17 & 0.03 & 0 \\
\hline $\begin{array}{l}\text { Soil15cm } \\
\text { Time23 }\end{array}$ & - & 0.13 & 0.26 & 0.18 & 0.02 & 0 & 0.08 & 0.24 & 0.17 & 0.03 & 0 \\
\hline $\begin{array}{l}\text { Soil15cm } \\
\text { Time24 }\end{array}$ & - & 0.13 & 0.26 & 0.18 & 0.02 & 0 & 0.08 & 0.23 & 0.17 & 0.03 & 0 \\
\hline
\end{tabular}


Table A-8. Descriptive statistics of soil moisture observations at $15 \mathrm{~cm}$ for timeline $15 \mathrm{July}-$ 31 August 2018.

\begin{tabular}{|c|c|c|c|c|c|c|c|c|c|c|c|}
\hline \multirow{3}{*}{\begin{tabular}{|l|} 
Contrast \\
Probe_id
\end{tabular}} & \multirow{3}{*}{\begin{tabular}{|l}
- \\
- \\
$N$
\end{tabular}} & \multicolumn{5}{|c|}{ Shallow Plastic } & \multicolumn{5}{|c|}{ Deep Plastic } \\
\hline & & \multicolumn{5}{|c|}{$1,10,12,13,14,28$} & \multicolumn{5}{|c|}{$1,10,12,14,28$ and 33} \\
\hline & & Min & Max & Mean & SD & $\begin{array}{l}\text { SE } \\
\text { Mean }\end{array}$ & Min & Max & Mean & SD & $\begin{array}{l}\text { SE } \\
\text { Mean }\end{array}$ \\
\hline Soil $15 \mathrm{~cm}$ & - & 0.12 & 0.26 & 0.17 & 0.02 & 0 & 0.06 & 0.26 & 0.17 & 0.03 & 0 \\
\hline \begin{tabular}{|l|} 
Soil15cm \\
Time1
\end{tabular} & - & 0.12 & 0.23 & 0.17 & 0.02 & 0 & 0.06 & 0.23 & 0.16 & 0.03 & 0 \\
\hline \begin{tabular}{|l|} 
Soil15cm \\
Time2
\end{tabular} & - & 0.12 & 0.24 & 0.17 & 0.02 & 0 & 0.06 & 0.24 & 0.16 & 0.03 & 0 \\
\hline \begin{tabular}{|l|} 
Soil15cm \\
Time3
\end{tabular} & - & 0.12 & 0.25 & 0.17 & 0.02 & 0 & 0.06 & 0.25 & 0.17 & 0.03 & 0 \\
\hline $\begin{array}{l}\text { Soil15cm } \\
\text { Time4 }\end{array}$ & - & 0.12 & 0.26 & 0.17 & 0.02 & 0 & 0.06 & 0.26 & 0.17 & 0.03 & 0 \\
\hline \begin{tabular}{|l|} 
Soil15cm \\
Time5
\end{tabular} & - & 0.12 & 0.26 & 0.17 & 0.02 & 0 & 0.06 & 0.26 & 0.17 & 0.03 & 0 \\
\hline \begin{tabular}{|l|} 
Soil15cm \\
Time6
\end{tabular} & - & 0.12 & 0.26 & 0.17 & 0.03 & 0 & 0.06 & 0.26 & 0.17 & 0.03 & 0 \\
\hline \begin{tabular}{|l|} 
Soil15cm \\
Time7
\end{tabular} & - & 0.12 & 0.26 & 0.17 & 0.03 & 0 & 0.06 & 0.26 & 0.17 & 0.03 & 0 \\
\hline $\begin{array}{l}\text { Soil15cm } \\
\text { Time8 }\end{array}$ & - & 0.12 & 0.25 & 0.17 & 0.03 & 0 & 0.06 & 0.25 & 0.17 & 0.03 & 0 \\
\hline \begin{tabular}{|l|} 
Soil15cm \\
Time9
\end{tabular} & - & 0.12 & 0.25 & 0.17 & 0.03 & 0 & 0.06 & 0.25 & 0.17 & 0.03 & 0 \\
\hline $\begin{array}{l}\text { Soil15cm } \\
\text { Time10 }\end{array}$ & - & 0.12 & 0.25 & 0.17 & 0.03 & 0 & 0.06 & 0.25 & 0.17 & 0.03 & 0 \\
\hline \begin{tabular}{|l|} 
Soil15cm \\
Time11
\end{tabular} & - & 0.12 & 0.25 & 0.17 & 0.03 & 0 & 0.06 & 0.25 & 0.17 & 0.03 & 0 \\
\hline \begin{tabular}{|l|} 
Soil15cm \\
Time12
\end{tabular} & - & 0.12 & 0.25 & 0.17 & 0.02 & 0 & 0.06 & 0.25 & 0.17 & 0.03 & 0 \\
\hline $\begin{array}{l}\text { Soil15cm } \\
\text { Time13 }\end{array}$ & - & 0.12 & 0.24 & 0.17 & 0.02 & 0 & 0.06 & 0.24 & 0.17 & 0.03 & 0 \\
\hline \begin{tabular}{|l|} 
Soil15cm \\
Time14
\end{tabular} & - & 0.12 & 0.24 & 0.17 & 0.02 & 0 & 0.06 & 0.24 & 0.17 & 0.03 & 0 \\
\hline $\begin{array}{l}\text { Soil15cm } \\
\text { Time15 }\end{array}$ & - & 0.12 & 0.25 & 0.17 & 0.02 & 0 & 0.06 & 0.25 & 0.17 & 0.03 & 0 \\
\hline $\begin{array}{l}\text { Soil15cm } \\
\text { Time16 }\end{array}$ & - & 0.12 & 0.25 & 0.17 & 0.02 & 0 & 0.06 & 0.25 & 0.17 & 0.03 & 0 \\
\hline $\begin{array}{l}\text { Soil15cm } \\
\text { Time17 }\end{array}$ & - & 0.12 & 0.24 & 0.17 & 0.02 & 0 & 0.07 & 0.24 & 0.17 & 0.03 & 0 \\
\hline $\begin{array}{l}\text { Soil15cm } \\
\text { Time18 }\end{array}$ & - & 0.12 & 0.24 & 0.17 & 0.02 & 0 & 0.07 & 0.24 & 0.17 & 0.03 & 0 \\
\hline $\begin{array}{l}\text { Soil15cm } \\
\text { Time19 }\end{array}$ & - & 0.12 & 0.24 & 0.17 & 0.02 & 0 & 0.07 & 0.24 & 0.17 & 0.03 & 0 \\
\hline $\begin{array}{l}\text { Soil15cm } \\
\text { Time20 }\end{array}$ & - & 0.12 & 0.24 & 0.17 & 0.02 & 0 & 0.07 & 0.24 & 0.17 & 0.03 & 0 \\
\hline
\end{tabular}




\begin{tabular}{|c|c|c|c|c|c|c|c|c|c|c|c|}
\hline Contrast & - & Shallov & N Plastic & & & & Deep P & Plastic & & & \\
\hline \multirow[t]{2}{*}{ Probe_id } & \multirow{2}{*}{$\begin{array}{l}- \\
\mathrm{N}\end{array}$} & \multicolumn{5}{|c|}{$1,10,12,13,14,28$} & \multicolumn{5}{|c|}{$1,10,12,14,28$ and 33} \\
\hline & & Min & Max & Mean & SD & \begin{tabular}{|l} 
SE \\
Mean
\end{tabular} & Min & Max & Mean & SD & $\begin{array}{l}\text { SE } \\
\text { Mean }\end{array}$ \\
\hline $\begin{array}{l}\text { Soil15cm } \\
\text { Time21 }\end{array}$ & - & 0.12 & 0.24 & 0.17 & 0.02 & 0 & 0.07 & 0.24 & 0.17 & 0.03 & 0 \\
\hline $\begin{array}{l}\text { Soil15cm } \\
\text { Time22 }\end{array}$ & - & 0.12 & 0.23 & 0.17 & 0.02 & 0 & 0.07 & 0.23 & 0.17 & 0.03 & 0 \\
\hline $\begin{array}{l}\text { Soil15cm } \\
\text { Time23 }\end{array}$ & - & 0.12 & 0.24 & 0.17 & 0.02 & 0 & 0.07 & 0.24 & 0.17 & 0.03 & 0 \\
\hline $\begin{array}{l}\text { Soil15cm } \\
\text { Time24 }\end{array}$ & - & 0.12 & 0.23 & 0.17 & 0.02 & 0 & 0.07 & 0.23 & 0.17 & 0.03 & 0 \\
\hline
\end{tabular}




\section{Appendix B. Classifiers and ROC analysis}

In this section we discuss standard background material concerning classification algorithms and measuring their performance.

Binary classifiers. A binary classifier is a process to label input data with yes or no.

Definition B.1. Let $U \subset \mathbb{R}^{n}$ be input data with $U$ a finite set. We say that $U$ is binary labeled data, or binary data for short, if there is a function $g: U \rightarrow\{$ no, yes\} referred to as the ground truth. Additionally, the labels no and yes must be ordered so that no is the negative label and yes is the positive label and we write no < yes.

Note that a similar definition can be made if the input data consist of categorical variables. This detail is omitted from Definition B.1 for simplicity.

Definition B.2. Let $U$ be binary data. A scoring function is a function $f: U \rightarrow \mathbb{R}$ that assigns to each input $x$ a score $f(x)$. A binary classifier is a scoring function $f$ together with a threshold $\tau \in \mathbb{R}$.

A binary classifier $(f, \tau)$ can be used to predict the ground truth. More precisely, the prediction $\hat{g}: U \rightarrow\{$ no, yes $\}$ is obtained from the binary classifier $(f, \tau)$ by defining $\hat{g}$ on an input $u \in U$ according to Equation (27).

$$
\hat{g}(u):=\left\{\begin{array}{l}
\text { yes, if } f(u) \geq \tau \\
\text { no, if } f(u)<\tau
\end{array}\right.
$$

The next step is to explain a way to measure the quality of a prediction $\hat{g}$ as defined above.

ROC analysis. Receiver operating characteristic (ROC) analysis is the primary tool for us to measure the prediction quality of binary classifiers. Here we review the basic concepts required for our purposes. For a more thorough treatment see (Fawcett 2006; Zou et al. 2011). Let $(f, \tau)$ be a binary classifier with input data, prediction $\hat{g}$ and ground truth $g$ as defined above. For a given input $u$ there are four possibilities, which are summarized in Definition B.3.

Definition B.3. Correct predictions, with $\hat{g}(u)=g(u)$, are referred to as true positive or true negative. An incorrect prediction with $\hat{g}(u)=$ no is 
called a false negative. An incorrect prediction with $\hat{g}(u)=$ yes is called a false positive (also called false alarm).

The confusion matrix, Table 26, is a way to organize how many times each of the four cases occurs. In the confusion matrix, TP stands for the number of true positives, and similarly for the other three cases. See Equation (28) for the precise definition of the confusion matrix entries.

Table B-1. Confusion matrix for a binary classifier.

\begin{tabular}{|c|c|c|}
\hline- & ground truth yes & ground truth no \\
\hline predicted yes & TP & FP \\
\hline predicted no & FN & TN \\
TP $:=\#\{u \in U: \hat{g}(u)=g(u)=$ yes $\}$ \\
TN $:=\{u \in U: \hat{g}(u)=g(u)=$ no $\}$ \\
FP $:=\#\{\boldsymbol{u} \in \boldsymbol{U}: \widehat{\boldsymbol{g}}(\boldsymbol{u})=$ yes, $\boldsymbol{g}(\boldsymbol{u})=$ no $\}$ \\
FN $:=\#\{u \in U: \hat{g}(u)=$ no, $g(u)=$ yes $\}$
\end{tabular}

Definition B.3. The true positive rate (TPR), also known as probability of detection (PD), is defined by the ratio of true positives in the left column of the confusion matrix:

$$
\text { TPR }:=\frac{\text { TP }}{\text { TP+FN }}
$$

Definition B.4. The false positive rate (FPR), also known as false alarm rate (FAR), is defined by the ratio of false positives in the right column of the confusion matrix:

$$
\text { FPR }:=\frac{\text { FP }}{\text { FP+TN }}
$$

As explained above, given a binary classifier $(f, \tau)$ and input data, we can construct a confusion matrix. ROC analysis provides a framework to analyze how the confusion matrix changes as $\tau$ varies and $f$ and $U$ stay fixed.

Definition B.5. Given a scoring function $f$ and binary input data $U$, a ROC graph or ROC curve is the set of $\tau$-indexed pairs $\mathcal{C}$ $:=\left\{\left(\mathrm{FPR}_{\tau}, \mathrm{TPR}_{\tau}\right)\right\}_{\tau}$ where $\mathrm{FPR}_{\tau}$ and $\mathrm{TPR}_{\tau}$ are the false positive rate and true positive rate, respectively, each obtained from the confusion matrix for the binary classifier $(f, \tau)$ on data $U$. 
There are several observations to note about the ROC curve. First, $\mathcal{C}$ is a continuous curve in the space $[0,1] \times[0,1] \subset \mathbb{R}^{2}$. Thus, the area under $\mathcal{C}$ (AUC) is well-defined and equal to the definite integral of a step function on the interval $[0,1]$. Lastly, the value of the AUC is in the interval $[0,1]$ and is equal to 1 if and only if the point $(0,1) \in \mathcal{C}$, which occurs if and only if $g=\hat{g}$ for every $\tau$.

Another way to think about $\mathcal{C}$ is in terms of type I and type II statistical errors in Figure 39. In this illustration, the blue curve represents the density function for the set of yes scores,

$$
\{f(u): g(u)=\text { yes }\}
$$

and the red curve represents the density function for the set of no scores

$$
\{f(u): g(u)=\text { no }\}
$$

The true positive rate is $1-\beta$ and the false positive rate is $\alpha$. As the threshold moves to the left the true positive rate increases ( $\beta$ decreases), but at a cost of an increase in the false positive rate ( $\alpha$ increases). If we plot the false positive rate $\alpha$ against the true positive rate $1-\beta$ as $\tau$ moves to the left, then we trace out the ROC curve $\mathcal{C}$.

In summary, the ROC curve $\mathcal{C}$ is the plot of the true positive rate against the false positive rate for a family of binary classifiers $\{(f, \tau): \tau \in \mathbb{R}\}$ on some fixed input data. The area under the ROC curve (AUC) is a common method to measure the overall performance distilled down to a single value in the unit interval $[0,1]$.

Figure B-1. A visualization of the dichotomy between $\alpha$ (type I error) and $\beta$ (type II error).

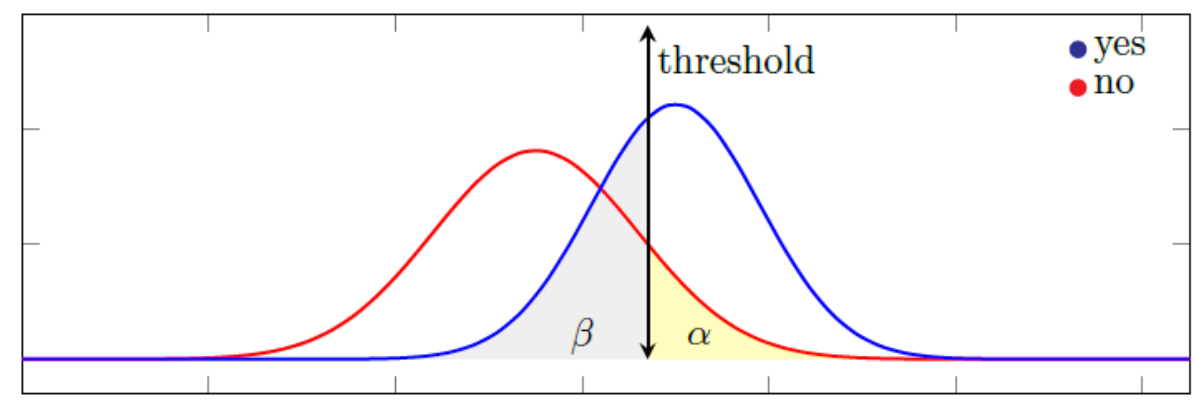




\section{Appendix C. Locations of targets and sensors}

In this section we include a summary of the environmental variables monitored and some plots of target and sensor locations to reference for variable naming conventions.

- The CS655 sensor locations are indicated in Appendix C in the moisture location figures. Each sensor yields three variables for temperature, electric conductivity, and soil moisture which we describe below.

- TmpXSY temperature measured in ${ }^{\circ} \mathrm{C}$

- ECONDXSY electric conductivity measured in $\mathrm{dS} / \mathrm{m}$

- VWCXSY soil moisture measured in \%

- $\mathrm{X}$ indicates the depth

- Y indicates the location

○ e.g., Tmp35SM8, ECOND35SM8, VWC35SM8, indicate the three variables for the CS655 sensor at $35 \mathrm{~cm}$ depth and location M8 in Figure C.4

- DTLayerX_RawTmp(Y) temperature measured in ${ }^{\circ} \mathrm{C}$

○ $\mathrm{X}$ indicates the depth

- Y indicates the location

○ e.g., DTLayer15_RawTmp(18) indicates $15 \mathrm{~cm}$ depth and location 18 in Figure C.2

- Plate1_ocm_Tmp, Plate2_5cm_Tmp heat plates measured in ${ }^{\circ} \mathrm{C}$

- FLX1, FLX2 heat flux measured in $\mathrm{W} / \mathrm{m}^{2}$

- ShortWave_In_Avg incoming short-wave radiation measured in $\mathrm{W} / \mathrm{m}^{2}$

- ShortWave_Reflect_Avg reflected short-wave radiation measured in $\mathrm{W} / \mathrm{m}^{2}$

- Weather data observed at the testing site at the ERDC-CRREL facility:

- AirTemp_Avg measured in ${ }^{\circ} \mathrm{C}$

- Pressure_Avg measured in mbar

- Rain_mm_Tot 24-hour rain accumulation total measured in $\mathrm{mm}$

- Rh_Avg relative humidity measured in \%

- WndSpeed_Avg measured in $\mathrm{m} / \mathrm{s}$

- WndDir_Avg measured in ${ }^{\circ}$

- Weather data observed at the nearby Lebanon airport:

○ cloud_cover measured in \%

- pressure measured in mbar

- rain_1h 1 hour rain totals measured in $\mathrm{mm}$ 
- rain_3h 3 hour rain totals measured in $\mathrm{mm}$

- snow_1h 1 hour snow totals measured in $\mathrm{mm}$

- snow_3h 3 hour snow totals measured in $\mathrm{mm}$

○ temp_min for the day measured in ${ }^{\circ} \mathrm{C}$

- temp_max for the day measured in ${ }^{\circ} \mathrm{C}$

- Sun position and time of day variables added using (Kennedy 2020):

- absolute_time measured in ms

- ms_after_previous_dawn measured in ms

- ms_after_previous_dusk measured in ms

o solar_azimuth, solar_elevation, solar_zenith

○ time_of_day measured in military time

- Variables for temperature, electric conductivity, and soil moisture which we describe below.

- TmpXSY temperature measured in ${ }^{\circ} \mathrm{C}$

- ECONDXSY electric conductivity measured in $\mathrm{dS} / \mathrm{m}$

- VWCXSY soil moisture measured in \%

- X indicates the depth

- Y indicates the location

○ e.g., Tmp35SM8, ECOND35SM8, VWC35SM8, indicate the three variables for the CS655 sensor at $35 \mathrm{~cm}$ depth and location M8 in Figure C.4

- DTLayerX_RawTmp(Y) temperature measured in ${ }^{\circ} \mathrm{C}$

- $\mathrm{X}$ indicates the depth

- Y indicates the location

- e.g., DTLayer15_RawTmp(18) indicates $15 \mathrm{~cm}$ depth and location 18 in Figure C. 2

- Plate1_ocm_Tmp, Plate2_5cm_Tmp heat plates measured in ${ }^{\circ} \mathrm{C}$

- FLX1, FLX2 heat flux measured in $\mathrm{W} / \mathrm{m}^{2}$

- ShortWave_In_Avg incoming short-wave radiation measured in $\mathrm{W} / \mathrm{m}^{2}$

- ShortWave_Reflect_Avg reflected short-wave radiation measured in $\mathrm{W} / \mathrm{m}^{2}$

- Weather data observed at the testing site at the ERDC-CRREL facility:

- AirTemp_Avg measured in ${ }^{\circ} \mathrm{C}$

- Pressure_Avg measured in mbar

- Rain_mm_Tot 24-hour rain accumulation total measured in $\mathrm{mm}$

○ Rh_Avg relative humidity measured in \%

- WndSpeed_Avg measured in $\mathrm{m} / \mathrm{s}$

- WndDir_Avg measured in ${ }^{\circ}$

- Weather data observed at the nearby Lebanon airport: 
- cloud_cover measured in \%

- pressure measured in mbar

0 rain_1h 1 hour rain totals measured in $\mathrm{mm}$

- rain_3h 3 hour rain totals measured in $\mathrm{mm}$

- snow_1h 1 hour snow totals measured in $\mathrm{mm}$

- snow_3h 3 hour snow totals measured in $\mathrm{mm}$

○ temp_min for the day measured in ${ }^{\circ} \mathrm{C}$

- temp_max for the day measured in ${ }^{\circ} \mathrm{C}$

- Sun position and time of day variables added using (Kennedy 2020):

○ absolute_time measured in ms

- ms_after_previous_dawn measured in ms

o ms_after_previous_dusk measured in ms

- solar_azimuth, solar_elevation, solar_zenith

o time_of_day measured in military time

\section{Temperature and moisture sensor locations}

This section details the location of temperature and moisture sensors in the test plot. We refer to the frame of reference used here as the sensor grid. This frame of reference conveys locations of sensors in relation to each other and in relation to target locations. It does not convey precise pixel locations within the IR images. We organize the figures by depth as follows:

- Figure $\mathrm{C}-1$

○ $5 \mathrm{~cm}$

- Temperature sensors and buried targets

- Figure C-2

○ $15 \mathrm{~cm}$

- Temperature and moisture sensors

- Figure C-3

○ $25 \mathrm{~cm}$

- Temperature sensors

- Figure $\mathrm{C}-4$

- $35 \mathrm{~cm}$

- Temperature and moisture sensors

- Figure C-5

○ $45 \mathrm{~cm}$

- Temperature sensors

- Figure C-6 
○ $55 \mathrm{~cm}$

- Temperature and moisture sensors

- Figure C-7

- $65 \mathrm{~cm}$

○ Temperature sensors

Figure C-1. Locations of temperature sensors at $5 \mathrm{~cm}$ with target locations in the sensor grid.
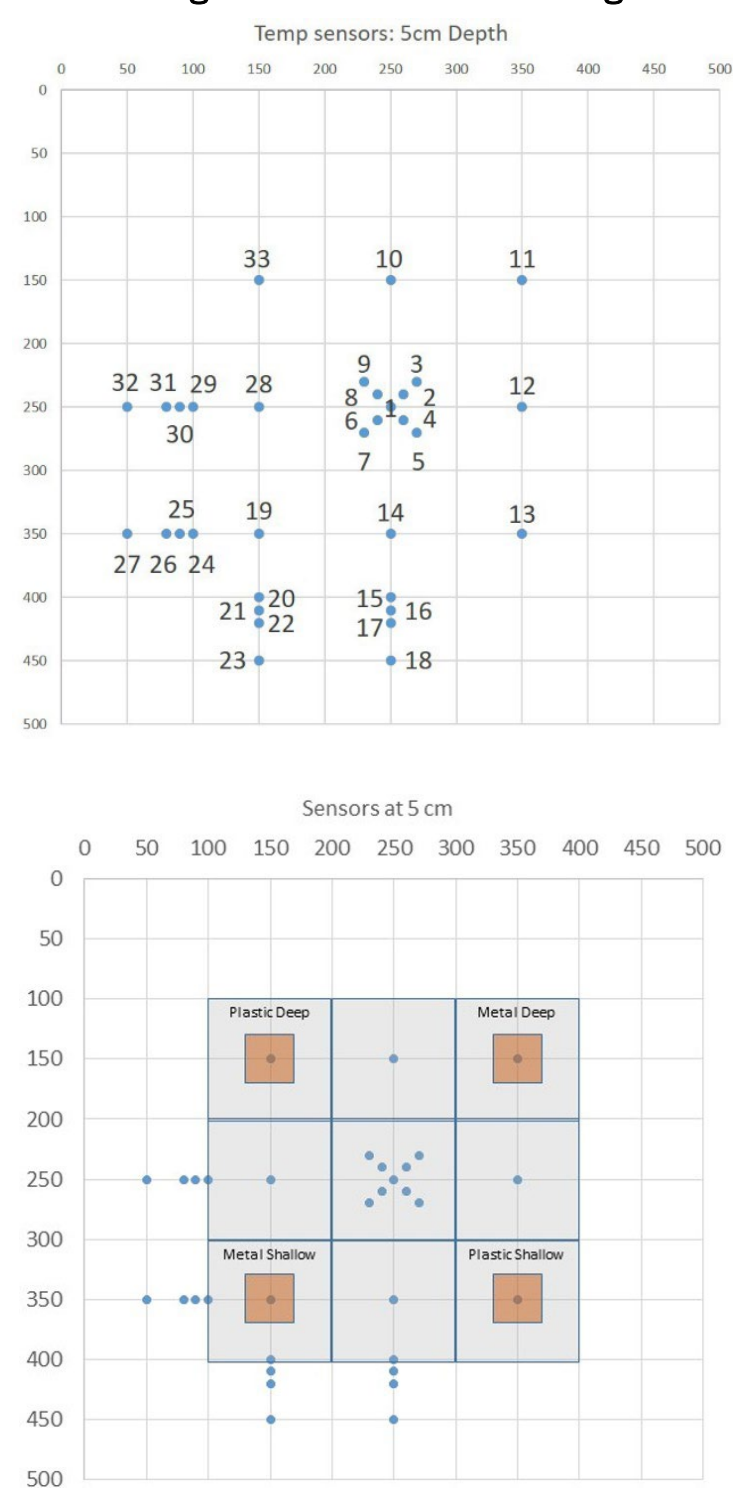
Figure C-2. Locations of temperature and moisture sensors at $15 \mathrm{~cm}$.
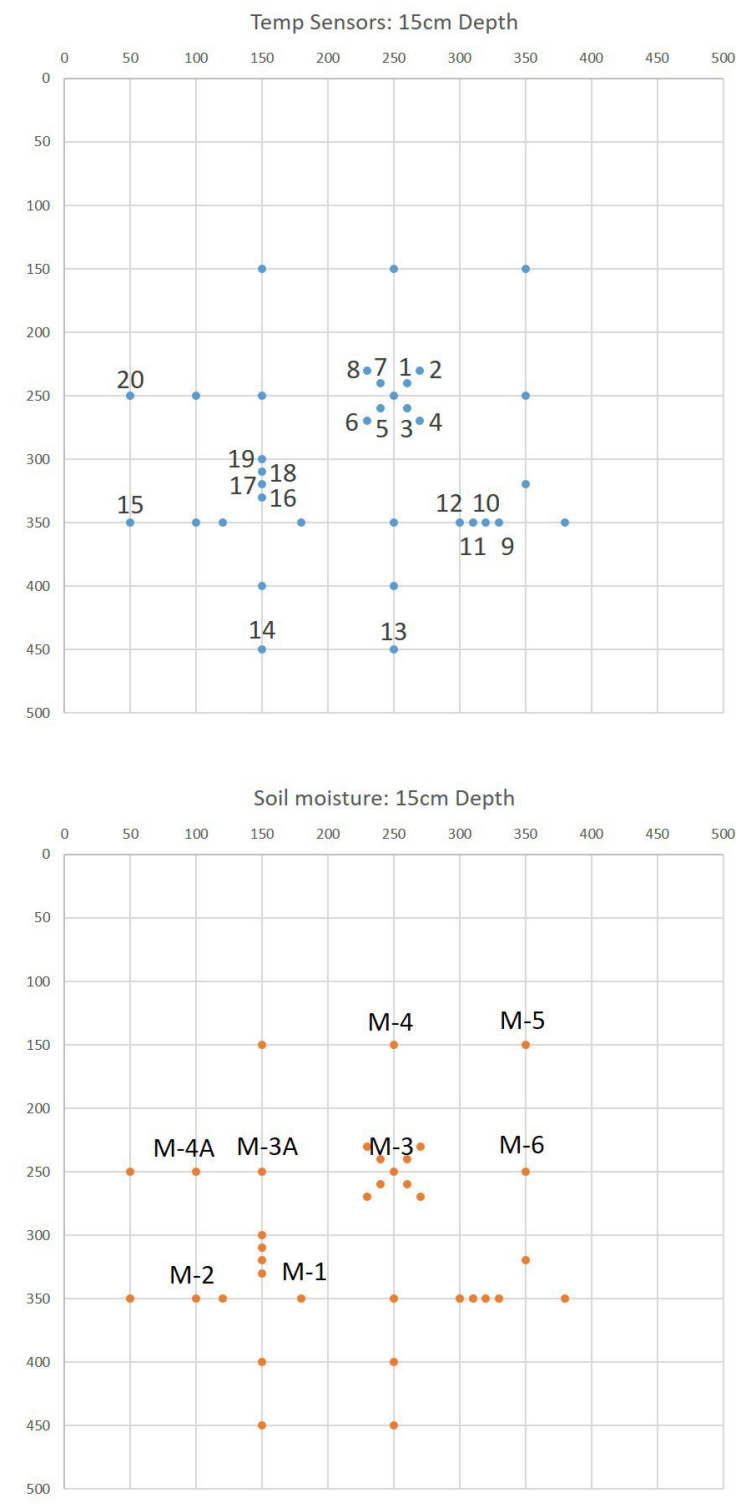
Figure C-3. Locations of temperature sensors at $25 \mathrm{~cm}$.

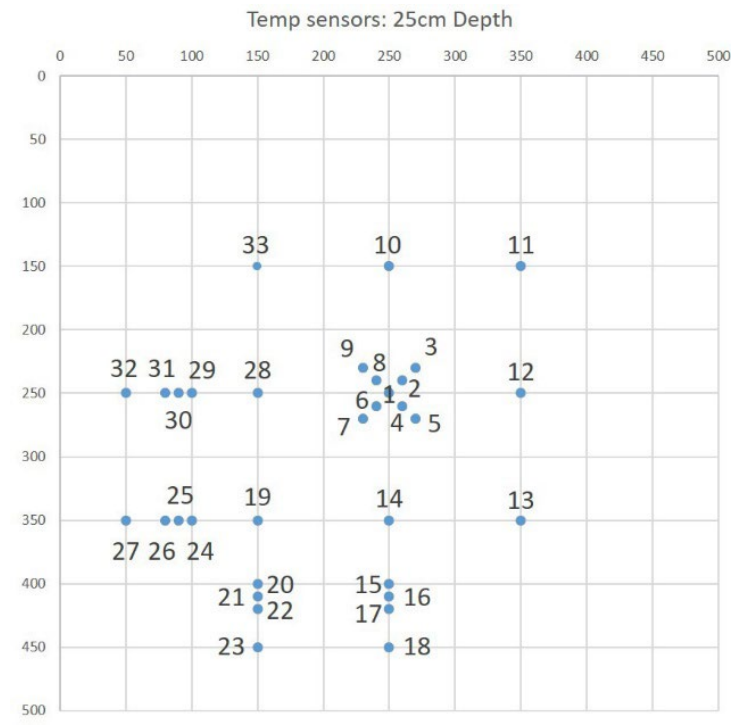


Figure C-4. Locations of temperature and moisture sensors at $35 \mathrm{~cm}$.

Figure C-5. Locations of temperature sensors at $45 \mathrm{~cm}$.

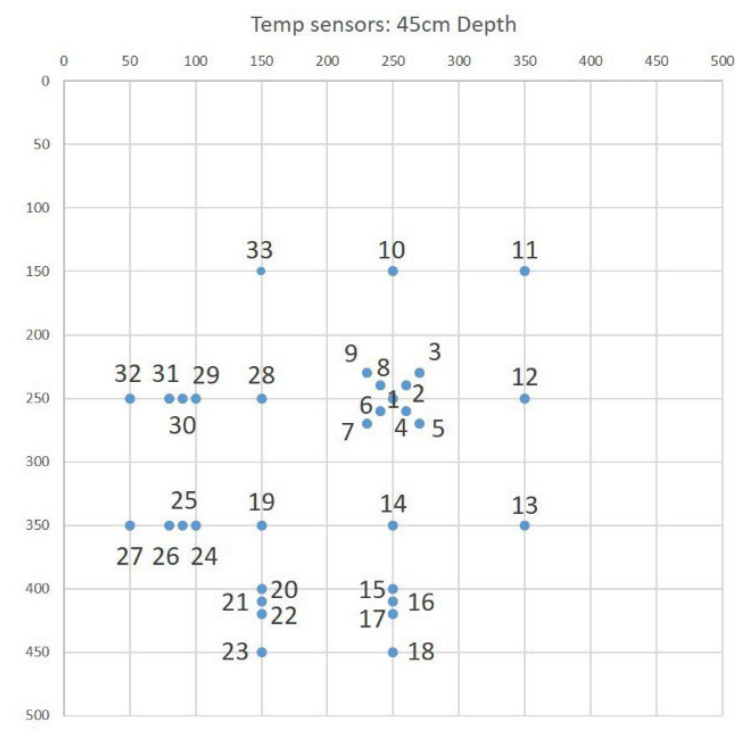


Figure C-6. Locations of temperature and moisture sensors at $55 \mathrm{~cm}$.
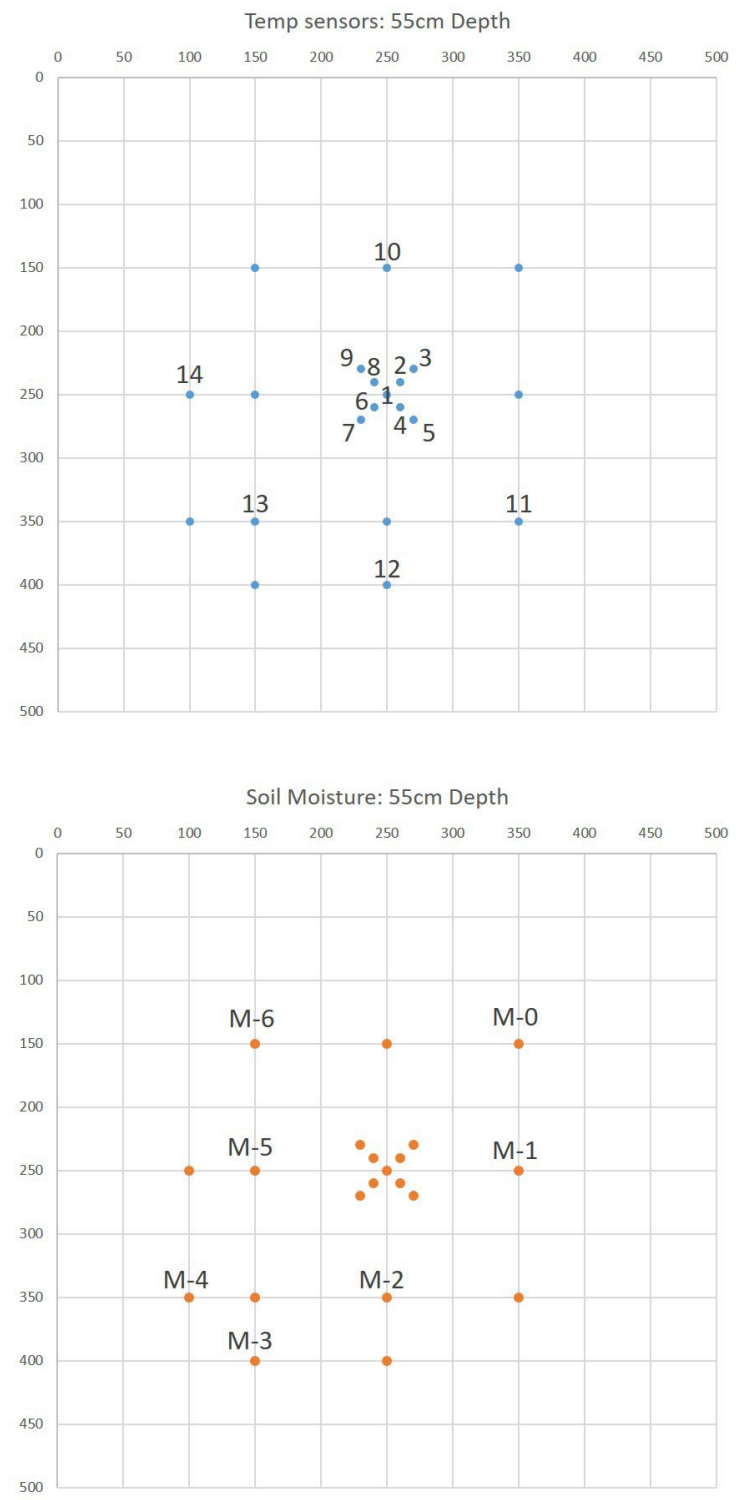
Figure C-7. Locations of temperature sensors at $65 \mathrm{~cm}$.

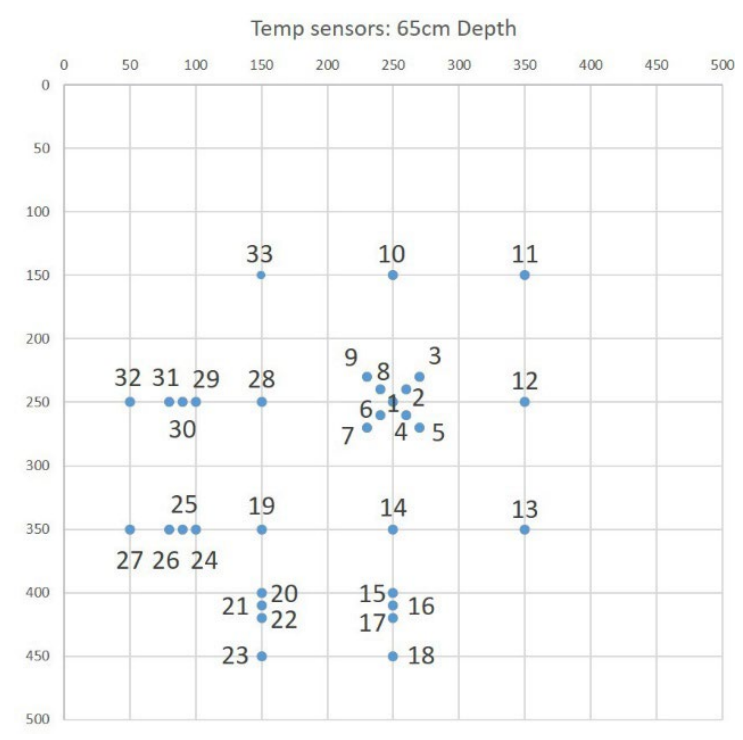




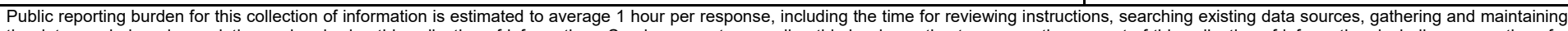

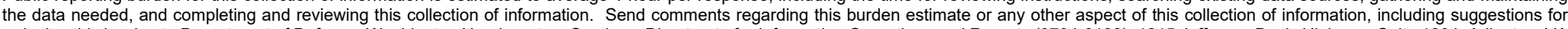

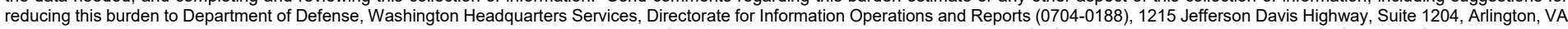

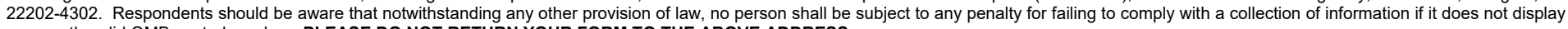
a currently valid OMB control number. PLEASE DO NOT RETURN YOUR FORM TO THE ABOVE ADDRESS.
1. REPORT DATE (DD-MM-YYYY)
2. REPORT TYPE
3. DATES COVERED (From - To)

March 2022

Technical Report / Final

\section{TITLE AND SUBTITLE}

Modernizing Environmental Signature Physics for Target Detection-Phase 3

5a. CONTRACT NUMBER

5b. GRANT NUMBER

5c. PROGRAM ELEMENT NUMBER 0602712A

\section{AUTHOR(S)}

5d. PROJECT NUMBER

468349

Jay L. Clausen, C. Felt, M. Musty, V. Truong, S. Frankenstein, A. Wagner, R. Affleck, S.

Peckham, and C. Williams

5e. TASK NUMBER

A1010

5f. WORK UNIT NUMBER

\section{PERFORMING ORGANIZATION NAME(S) AND ADDRESS(ES)}

8. PERFORMING ORGANIZATION REPORT NUMBER

U.S. Army Engineer Research and Development Center (ERDC)

Cold Regions Research and Engineering Laboratory (CRREL)

ERDC/CRREL TR-22-4

72 Lyme Road

Hanover, NH 03755-1290

9. SPONSORING / MONITORING AGENCY NAME(S) AND ADDRESS(ES)

U.S. Army Combat Capabilities Development Command

Night Vision and Electronic Sensors Directorate (NVESD)

AMSEL-RD-NV-RM-OPS

10221 Burbeck Road

Ft. Belvoir, VA 22060-5806

12. DISTRIBUTION / AVAILABILITY STATEMENT

Approved for public release; distribution is unlimited.

\section{SUPPLEMENTARY NOTES}

\section{ABSTRACT}

The present effort (Phase 3) builds on our previously published prior efforts (Phases 1 and 2), which examined methods of determining the probability of detection and false alarm rates using thermal infrared for buried object detection.

Environmental phenomenological effects are often represented in weather forecasts in a relatively coarse, hourly resolution, which introduces concerns such as exclusion or misrepresentation of ephemera or lags in timing when using this data as an input for the Army's Tactical Assault Kit software system. Additionally, the direct application of observed temperature data with weather model data may not be the best approach because metadata associated with the observations are not included. As a result, there is a need to explore mathematical methods such as Bayesian statistics to incorporate observations into models.

To better address this concern, the initial analysis in Phase 2 data is expanded in this report to include (1) multivariate analyses for detecting objects in soil, (2) a moving box analysis of object visibility with alternative methods for converting FLIR radiance values to thermal temperature values, (3) a calibrated thermal model of soil temperature using thermal IR imagery, and (4) a simple classifier method for automating buried object detection.

15. SUBJECT TERMS

Earth temperature, Environmental conditions, Infrared detectors, Infrared imaging, Soil science, Military surveillance

\section{SECURITY CLASSIFICATION OF:}

\section{a. REPORT}

Unclassified

\section{b. ABSTRACT}

Unclassified

\section{c. THIS PAGE}

Unclassified

\begin{tabular}{c|c}
$\begin{array}{c}\text { 17. LIMITATION } \\
\text { OF ABSTRACT }\end{array}$ & $\begin{array}{c}\text { 18. NUMBER } \\
\text { OF PAGES }\end{array}$ \\
None & 159 \\
\hline
\end{tabular}

19a. NAME OF RESPONSIBLE PERSON

19b. TELEPHONE NUMBER (include area code) 